\title{
Radio Resource Allocation over Fading Channels under Statistical Delay Constraints
}

\author{
Khoa Tran Phan
}

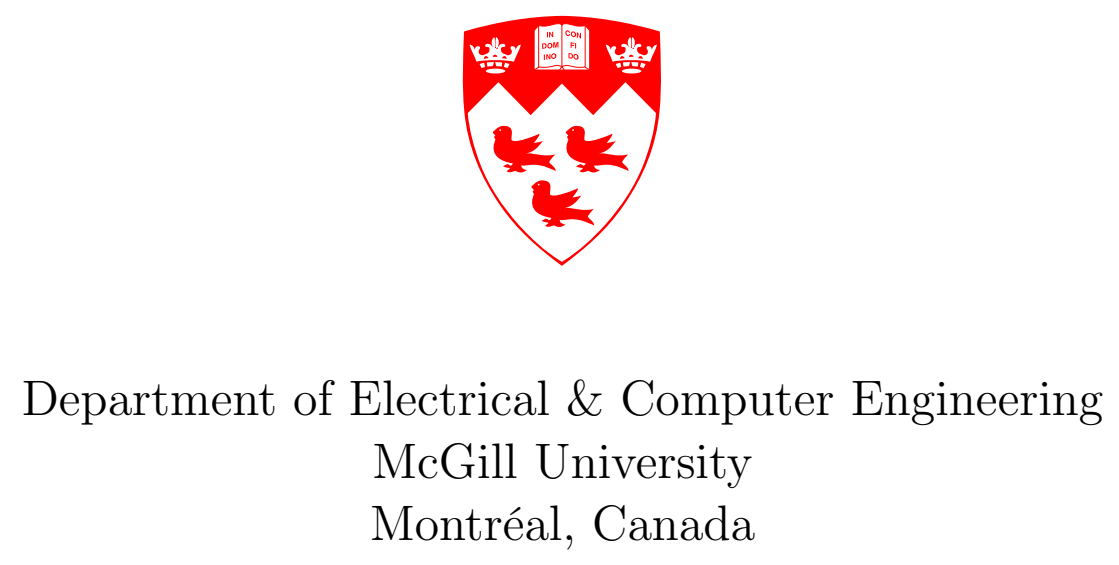

December 2016

A thesis submitted to McGill University in partial fulfillment of the requirements for the degree of Doctor of Philosophy.

(c) 2016 Khoa Tran Phan 
To my parents, my wife Dăng Vy, and our daughter Lamma With all my love. 


\begin{abstract}
Wireless data transmission suffers from the fading nature of wireless channels, where the instantaneous channel conditions and hence transmission rates randomly fluctuate over time. Consequently, data arrivals at each transmitter might not be transmitted instantly. To cope with this situation, the transmitter employs buffer to store the data temporarily for later transmission. While data buffering enables more efficient radio resource allocation to opportunistically select the favorable fading conditions for transmission, it introduces queuing delay that needs to be controlled in order to meet the end-to-end delay quality-ofservice (QoS) requirements in supporting delay-sensitive communications. In this thesis, we study and develop radio resource allocation schemes for buffer-aided communications over wireless fading channels under statistical delay constraints. Using the buffering capability as a means to exploit the fading diversity, the nodes (source and relay) perform resource allocation, and adapt their transmissions to the channel state information (CSI) in order to enhance the system throughput while maintaining the statistical delay QoS requirements in terms of upper-bounded average delay or delay-outage probability.

The thesis starts by considering a source-destination communications link over a fading channel with data arriving at the source transmission buffer. In the first scenario, the source is assumed to have a maximum power constraint and an average delay constraint. We consider admission control applied on random data arrivals to the source buffer in order to avoid constraint violation, and study the joint optimal data admission control and power allocation (AC-PA) problem for throughput maximization. In the second scenario, we consider an energy-harvesting $(\mathrm{EH})$ source, where random amounts of energy are harvested from renewable energy sources, and stored in a battery during the course of data transmission. In every transmission time slot, the source is constrained to use at most the amount of energy currently stored. We then explore optimal power allocation problems for such EH systems under average delay or delay-outage constraints. We formulate the problems as infinite horizon constrained Markov decision process (MDP) problems, which incorporate the random variations of the fading channel, data arrival, and EH processes. A novel solution approach based on post-decision state-value function is proposed to study the properties of the optimal solutions. We also propose online allocation algorithms when the statistical knowledge of the random processes is unknown, which is typical in real-life communications. Illustrative results demonstrate the effectiveness of the proposed algorithms over existing approaches
\end{abstract}


under similar power and delay constraints.

The thesis continues with a source-relay-destination communications link over fading channels with buffers available at both source and relay, as part of a multi-hop network. We investigate and develop optimal resource allocation schemes for this 3-node buffer-aided relaying system to maximize its effective capacity under a (end-to-end) delay-outage constraint, with special emphasis on relay roles. We consider the typical half-duplex (HD) relay operation in which the source-relay and relay-destination links cannot simultaneously transmit on the same frequency slot. In particular, under the established effective capacity maximization criterion and delay-outage constraint, we study the optimal adaptive link selection relaying problem jointly with both fixed and adaptive power allocation schemes. Such HD relaying avoids self-interference (SI) at the expense of low spectral efficiency. We next consider the full-duplex (FD) relay that can support simultaneous transmissions of both source-relay and relay-destination links over the same frequency slot to enhance the spectral efficiency in presence of non-zero residual self-interference (SI) due to non-ideal SIcancellation. We formulate the corresponding optimal power allocation problems for both cases of available knowledge of the channel state information at transmitter (CSIT): instantaneous or statistical. We employ asymptotic delay analysis to transform the delay-outage constraint into more tractable constraints, so that the resulting constrained optimization problems can be solved by a Lagrangian approach. Numerical studies have demonstrated the effective capacity gains of the proposed relaying schemes over existing schemes. With instantaneous CSIT, FD relaying is more beneficial than HD relaying. On the other hand, with statistical CSIT, FD relaying is better than HD-relaying only when the residual SI is below certain level (i.e., with good SI-cancellation). 


\section{Sommaire}

La transmission sans fil de données souffre de l'évanouissement du canal sans fil, ou les conditions instantanées du canal et donc les débits de transmission fluctuent au cours du temps. En conséquence, les données arrivant à chaque émetteur ne peuvent pas être transmises instantanément. Pour remédier à cette situation, l'émetteur utilise une mémoire-tampon pour sauvegarder les données temporairement afin d'une transmission ultérieur. Bien que la mise en mémoire-tampon de données permet une allocation plus efficace des ressources radio pour sélectioner les bonnes conditions de propagation à transmission par une façon opportuniste, elle introduit de délai dans la file d'attente qui doit être contrôlée afin de satisfaire la qualité de service (QoS) requise dans les communications sensibles au délai. Dans cette thèse, nous étudions et développons des schémas d'allocation de ressource sur des canaux sans fil à évanouissement sous contraintes de délai statistique. En utilisant la capacité de stockage comme moyen pour exploiter la diversité de l'évanouissement, les noeuds (source et relai) effectuent de l'allocation de ressource et adaptent leur transmissions à l'état du canal (CSI) afin de booster le débit du système en maintenant la requise QoS du délai, en termes du délai moyen ou la probabilité de panne en délai, limité par une borne supérieure.

La thèse commence par l'examen d'une liaison de communication entre une source et une destination sur un canal à évanouissement avec des données arrivant dans la mémoire tampon d'émission de la source. Dans le premier scénario, la source est supposée d'avoir une contrainte sur la puissance maximale et le délai moyen. Nous considérons le contrôle d'admission appliquée sur les arrivées de données aléatoires dans la mémoire tampon de la source afin d'éviter une violation des contraintes, et d'étudier le problème de contrôle optimal de l'admission de données et d'allocation de puissance conjointe (AC-PA) pour maximiser le débit. Dans le deuxième scénario, nous considérons une source à récolte d'énergie $(\mathrm{EH})$, où des quantités aléatoires d'énergie sont récoltées à partir de sources d'énergie renouvelables, et stockés dans une batterie au cours de la transmission de données. Dans chaque créneau temporel de transmission, la source est contrainte d'utiliser au maximum la quantité d'énergie actuellement stockée. Nous explorons ensuite les problèmes optimaux d'allocation de puissance pour de tels systèmes EH sous des contraintes de délai moyen ou de délai de dépassement. Nous formulons les problèmes sous une forme de processus de décision de Markov à horizon infini (MDP), qui intègrent les variations aléatoires de l'évanouissement du canal, l'arrivée de données, et les processus EH. Une nouvelle approche basée sur la fonction de 
valeur d'état post-décision est proposé afin étudier les propriétés des solutions optimales. Nous proposons également des algorithmes d'allocation lorsque la connaissance statistique des processus aléatoires est inconnue, ce qui est typique dans les communications réels. Les résultats illustratifs démontrent la supériorité des algorithmes proposés sur les approches existantes sous les contraintes et délai et de puissance.

La thèse se poursuit avec l'examen d'un lien de communication à trois noeuds: source, relais, destination, sur des canaux d'évanouissement avec des tampons disponibles à la source au le relais. Nous étudions et développons des schémas optimaux d'allocation des ressources pour ce lien à 3 noeuds afin de maximiser sa capacité effective sous une contrainte de panne en délai avec un accent particulier sur les rôles de relais. Nous considérons un relai opérant en semi-duplex (HD), òu la communication bidirectionnelle entre la source et le relais ne peut pas s'effectuer simultanément sur la même fréquence. En particulier, selon le critère de maximisation de la capacité effective et la contrainte de délai de dépassement, nous étudions la sélection optimale des liaisons adaptatives en collaboration avec des schémas d'allocation de puissance fixe et adaptative. Le relais HD évite auto-interférence (SI) au détriment de la faible efficacité spectrale. Nous examinons ensuite le relais operant en duplex intégral (FD) qui peut supporter des transmissions simultanées entre le source et le relais et entre le relais et la destination sur la même fréquence pour améliorer l'efficacité spectrale en présence de la non-zéro SI résiduelle dû à raison l'annulation de SI non-idéale. Nous formulons les problèmes de répartition de puissance optimale pour les deux cas de connaissance de l'information d'état de canal à l'émetteur (CSIT): instantanée et statistique. Nous employons l'analyse du délai asymptotique pour transformer la contrainte de panne en délai à des contraintes plus traitables, pourque les problèmes d'optimisation sous contrainte peuvent être résolus par une approche lagrangienne. Des études numériques ont démontré les gains de capacité effective des schémas à relais proposés par rapport aux systèmes existants. Avec la CSIT instantanée, le relais FD est plus bénéfique que le relais HD. D'autre part, avec la CSIT statistique, le relais FD est meilleur que le relais HD seulement quand la SI résiduelle est inférieure à un certain niveau (par exemple, avec une bonne annulation de SI). 


\section{Acknowledgments}

First and foremost, I would like to gratefully acknowledge my supervisor, Professor Tho LeNgoc for his strong guidance, and contributions in the development of the research in this thesis. He guided me into this challenging and interesting research area and encouraged me to pursue concrete results. Without his outstanding research vision, advice, and willingness to provide funding, the thesis is impossible to complete. Working with him is an enjoyable and precious experience.

I want to thank Professor Long Le of INRS, University of Quebec for kind help in my professional development. I would like to express my gratitude to Professors Michael Rabbat and Hannah Michalska for serving on my Ph.D. committee and providing valuable advice on my research. I would also like to thank Professor Victor C.M. Leung of University of British Columbia, and Professor Benoit Champagne of McGill University for serving as the examiners to my Ph.D. dissertation.

My friends at the Broadband Communications Research Lab have made my life at McGill exciting and memorable. I especially thank Imtiaz for our successful collaboration, which leads to the work presented in Chapter 4. Many thanks to Ahmed for helping me with the French translation.

Finally, this dissertation is dedicated to my family members for their love, sacrifice and unconditional support. 


\section{Contents}

1 Introduction $\quad 1$

1.1 Motivation . . . . . . . . . . . . . . . . . . . 1

1.2 Resource Allocation under Delay Constraints . . . . . . . . . . . . . . . 3

1.3 Thesis Objectives, Contributions, and Outline . . . . . . . . . . . . . . 4

2 Power Allocation over Fading Channels under Delay Constraints: A Literature Review $\quad 9$

2.1 Average Delay Constraint . . . . . . . . . . . . . . . . . . . . . 10

2.2 Delay-Outage Constraint . . . . . . . . . . . . . . . . . . . . . . 11

2.2.1 Asymptotic Delay Analysis . . . . . . . . . . . . . . . . . . . . 12

2.2.2 Effective Capacity . . . . . . . . . . . . . . . 13

2.2.3 EC-based resource allocation and performance analysis . . . . . . . . 14

2.3 Energy Harvesting Communications Systems . . . . . . . . . . . . . . . . . . 16

2.4 Buffer-Aided Relaying Communications . . . . . . . . . . . . . . . . . 18

2.4.1 Half-duplex Relaying with Adaptive Link Selection . . . . . . . . . . 19

2.4 .2 Full-duplex Relaying . . . . . . . . . . . . . . . . . . . 23

2.5 Chapter Summary . . . . . . . . . . . . . . . . . . . . . . . . 24

3 Joint Data Admission Control and Power Allocation over Fading Channel under Average Delay Constraint $\quad 25$

3.1 System Model and Problem Formulation . . . . . . . . . . . . . . . . 26

3.1.1 Model Description . . . . . . . . . . . . . . . . . . 26

3.1.2 Problem Formulation . . . . . . . . . . . . . . . . . . . . . 28

3.1.3 Optimal Throughput- Delay Trade-off . . . . . . . . . . . . . . 28

3.2 Joint Data Admission Control- Power Allocation . . . . . . . . . . . . . . . . 31 
3.2.1 MDP-based Optimal Solution . . . . . . . . . . . . . . . 31

3.2.2 Post-decision State-value Function Approach . . . . . . . . . . . . . . 32

3.2 .3 Structural Results . . . . . . . . . . . . . . . . . . 32

3.2 .4 Online Algorithm . . . . . . . . . . . . . . . . 34

3.3 Illustrative Results . . . . . . . . . . . . . . . . . . 36

3.3 .1 Settings . . . . . . . . . . . . . . . 36

3.3 .2 Numerical Results . . . . . . . . . . . . . . . . . 37

3.4 Chapter Summary . . . . . . . . . . . . . . . . . . . . . . 39

4 Power Allocation with Energy Harvesting over Fading Channel under Statistical Delay Constraints $\quad 41$

4.1 System Model and Problem Formulations . . . . . . . . . . . . . . . . . 42

4.1 .1 Model Description . . . . . . . . . . . . . . . . . . 42

4.1 .2 Problem Formulations . . . . . . . . . . . . . . . . 45

4.2 Power Allocation under Average Delay Constraint . . . . . . . . . . . . . . 45

4.2.1 Optimal Allocation Solution _................ 45

4.2 .2 Online Algorithm . . . . . . . . . . . . . . . . . . . 49

4.2 .3 Baseline Transmission Schemes . . . . . . . . . . . . . . . 51

4.3 Power Allocation under Delay-Outage Constraint . . . . . . . . . . . . . . 53

4.3.1 Effective Capacity Maximization . . . . . . . . . . . . . . 53

4.3 .2 Online Algorithm . . . . . . . . . . . . . . 56

4.3.3 Baseline Transmission Schemes . . . . . . . . . . . . 56

4.4 Illustrative Results . . . . . . . . . . . . . . . . . . . . 58

4.4 .1 Average Delay Constraint . . . . . . . . . . . . . . . 59

4.4 Delay-outage Constraint . . . . . . . . . . . . . . . 60

4.4.3 Average Delay versus Delay-outage Constraints . . . . . . . . . . 63

4.4.4 Convergence Study of the Online Algorithms . . . . . . . . . . . . . . 65

4.5 Chapter Summary . . . . . . . . . . . . . . . . . . 67

5 Resource Allocation for Buffer-Aided Half-Duplex Relaying under Delay$\begin{array}{ll}\text { Outage Constraint } & 68\end{array}$

5.1 System Model and Problem Formulation . . . . . . . . . . . . . . . . 69

5.1 .1 Model Description ....................... 69 
5.1 .2 Problem Formulation . . . . . . . . . . . . . . . . . . 71

5.2 Delay-Outage Constraint Transformation . . . . . . . . . . . . . . . . 71

5.2.1 Asymptotic Delay Analysis for Buffer-aided Relaying Network . . . . 71

5.2.2 Delay-outage Constraint Transformation . . . . . . . . . . . . . 73

5.3 Adaptive Link Scheduling with Fixed Power Allocation . . . . . . . . . . . . 73

5.3 .1 Optimal Solution . . . . . . . . . . . . . . . . 73

5.3 .2 Special Cases . . . . . . . . . . . . . . . . 76

5.3.3 Comparison Benchmarks . . . . . . . . . . . . . . 82

5.4 Adaptive Link Scheduling with Adaptive Power Allocation . . . . . . . . . . 85

5.4 .1 Optimal Solution . . . . . . . . . . . . . . . 85

5.4 .2 Special Cases . . . . . . . . . . . . . . . . . 89

5.4.3 Resource Allocation Algorithm over Unknown Fading Links . . . . . 90

5.5 Illustrative Results . . . . . . . . . . . . . . . . . . . . . . . . . 91

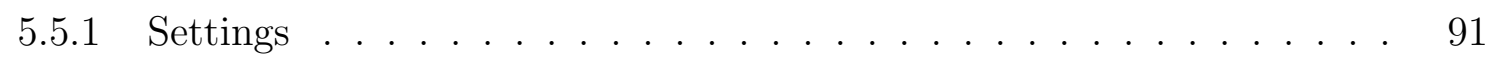

5.5 .2 Fixed Power Allocation . . . . . . . . . . . . . . . . . 91

5.5.3 Adaptive Power Allocation . . . . . . . . . . . . . . . . . 96

5.6 Chapter Summary . . . . . . . . . . . . . . . . . . . . . . . . . . . 98

6 Power Allocation for Buffer-Aided Full-Duplex Relaying with Imperfect Self-Interference Cancellation under Delay-Outage Constraint 99

6.1 System Model and Problem Formulations . . . . . . . . . . . . . . . . . 101

6.1.1 Model Description ..................... 101

6.1 .2 Problem Formulation . . . . . . . . . . . . . . . . . 103

6.2 Adaptive Power Allocation with Instantaneous CSIT . . . . . . . . . . . . . 104

6.2 .1 Optimal Solution . . . . . . . . . . . . . . . . . 104

6.2 .2 Special Cases . . . . . . . . . . . . . . . . . 107

6.2.3 Iterative Power Allocation Algorithm . . . . . . . . . . . . . . . . . . 109

6.3 Static Power Allocation with Statistical CSIT . . . . . . . . . . . . . . . . . 110

6.3.1 Optimal Solution . . . . . . . . . . . . . . 110

6.3.2 Properties of the Optimal Solution . . . . . . . . . . . . . 112

6.3.3 Special Cases . . . . . . . . . . . . . . . . . . . . 113

6.4 Power Allocation in Non-Buffer Full-Duplex Relaying . . . . . . . . . . . . . 114

6.4.1 Adaptive Power Allocation . . . . . . . . . . . . . . . . 115 
6.4 .2 Static Power Allocation . . . . . . . . . . . . . . . . 115

6.5 Illustrative Results and Discussions . . . . . . . . . . . . . . . . . . 116

6.5 .1 Settings . . . . . . . . . . . . . . . 116

6.5.2 Effects of SI Cancellation . . . . . . . . . . . . . . 117

6.5.3 Effects of Total Power Constraint . . . . . . . . . . . . . . . . . . . . 121

6.5.4 Effects of the Delay Constraint . . . . . . . . . . . . . . . . . . 124

6.5.5 Summary of Key Results . . . . . . . . . . . . . . . . . . . 125

6.6 Chapter Summary . . . . . . . . . . . . . . . . . . 126

7 Conclusions and Future Works $\quad 128$

7.1 Summary . . . . . . . . . . . . . . . . . . . . 128

7.2 Potential Future Works . . . . . . . . . . . . . . . . . . . . 130

$\begin{array}{ll}\text { References } & 133\end{array}$ 


\section{List of Figures}

2.1 Dynamic queue with arrival $\{a[t]\}$ and service $\{r[t]\}$ processes. . . . . . . 12

2.2 A source-destination communications link with EH transmitter. . . . . . . 17

2.3 Relaying in wireless cellular communications. . . . . . . . . . . . . . . 18

2.4 Buffer-aided relaying model. . . . . . . . . . . . . . . . . . . . . . 20

3.1 A source-destination communications link with source buffer. . . . . . . . . . 27

3.2 Optimal power- queue length trade-off. . . . . . . . . . . . . . . . . . 37

3.3 Optimal throughput- queue length trade-off for $\bar{P}^{\max }=4.5 \ldots \ldots \ldots$

3.4 Optimal throughput- queue length trade-off: $\bar{P}^{\max }=3 \ldots \ldots$. . . . . . 39

4.1 A source-destination communications link with EH transmitter. . . . . . . . 43

4.2 Throughput versus average queue length $\bar{Q}^{\max } \ldots \ldots \ldots \ldots$. . . . . . . . 59

4.3 Throughput versus average harvested energy $\bar{E}$. . . . . . . . . . . . . . . 61

4.4 Effective capacity versus $\zeta_{Q} \ldots \ldots \ldots \ldots \ldots \ldots$

4.5 Effective capacity versus average harvested energy $\bar{E}$. . . . . . . . . . . 63

4.6 Online allocation algorithm convergence: Effective capacity. . . . . . . . . 65

4.7 Online allocation algorithm convergence: Average power. . . . . . . . . . . . 66

$5.1 \quad$ B-HD relaying model. . . . . . . . . . . . . . . . . . . . . . . . . . . . 69

5.2 Multiplier $\xi$ versus delay exponent $\theta$ with $\mathrm{SNR}_{2}=10 \mathrm{~dB}$. . . . . . . . . 81

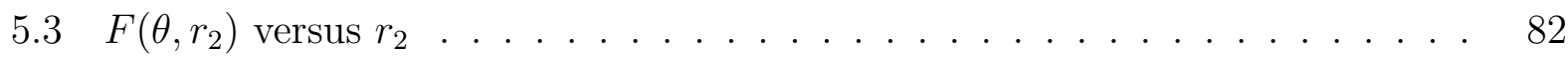

5.4 Optimal power allocation $P_{1}^{*}[t]$ versus $h_{1}[t] \ldots \ldots \ldots \ldots$

5.5 Effective capacity versus scaling parameter $v \ldots \ldots \ldots$. . . . . . . 92

5.6 Effective capacity versus $P_{1} \ldots \ldots \ldots \ldots \ldots$

5.7 Effective capacity versus $\mathrm{SNR}_{1} \ldots \ldots \ldots \ldots \ldots$ 
5.8 Effective capacity versus $\zeta_{Q} \ldots \ldots \ldots \ldots \ldots \ldots$

5.9 Effective capacity versus $\bar{P}^{\max } \ldots \ldots \ldots \ldots$. . . . . . . . . . . . . 96

5.10 Effective capacity versus $\zeta_{Q} \ldots \ldots \ldots \ldots$. . . . . . . . . . . 97

6.1 B-FD relaying model. . . . . . . . . . . . . . . . . . . . . . . . . . . . 101

6.2 Optimal static power allocation versus $\beta \ldots \ldots \ldots \ldots \ldots$. . . . . . . . 118

6.3 Effective capacity versus $\beta$ with $\zeta_{Q}=1 \ldots \ldots \ldots \ldots \ldots$. . . . . . 119

6.4 Effective capacity versus $\beta$ with $\zeta_{Q}=10^{-4} \ldots \ldots \ldots \ldots$. . . . . . 120

6.5 Effective capacity versus $P^{\max }$ with $\zeta_{Q}=1 \ldots \ldots \ldots \ldots \ldots$. . . . . . 122

6.6 Effective capacity versus $P^{\max }$ with $\zeta_{Q}=10^{-4} \ldots \ldots \ldots \ldots$. . . . . . 123

6.7 Effective capacity versus $\zeta_{Q}$ with adaptive power allocation. . . . . . . . . . 124

6.8 Effective capacity versus $\zeta_{Q}$ with static power allocation. . . . . . . . . . . 126 


\section{List of Tables}

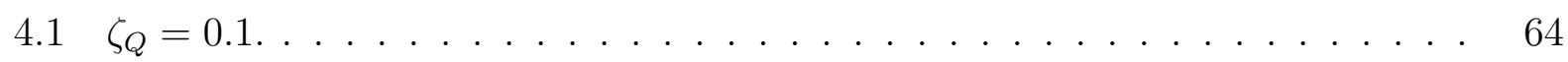

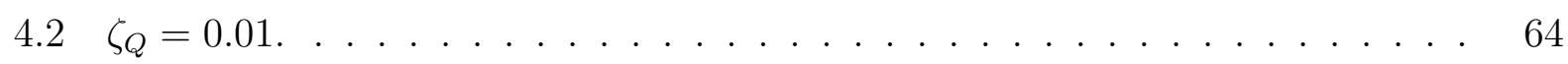




\section{List of Acronyms}

$\begin{array}{ll}\text { 5G } & \text { Fifth-generation } \\ \text { AC-PA } & \text { Admission control- power allocation } \\ \text { AWGN } & \text { Additive white Gaussian noise } \\ \text { B-FD } & \text { Buffer-aided full-duplex relaying } \\ \text { B-FD-APA } & \text { Buffer-aided full-duplex relaying with adaptive power allocation } \\ \text { B-FD-SPA } & \text { Buffer-aided full-duplex relaying with static power allocation } \\ \text { B-HD } & \text { Buffer-aided half-duplex relaying } \\ \text { B-HD-ALS } & \text { Buffer-aided half-duplex relaying with adaptive link scheduling } \\ \text { B-HD-FLS } & \text { Buffer-aided half-duplex relaying with fixed link scheduling } \\ \text { CSI } & \text { Channel state information } \\ \text { CSIT } & \text { Channel state information at the transmitters } \\ \text { DT } & \text { Direct transmission } \\ \text { E2E } & \text { End-to-end } \\ \text { EC } & \text { Effective capacity } \\ \text { ECCA } & \text { Energy constrained control algorithm } \\ \text { EH } & \text { Energy harvesting } \\ \text { FD } & \text { Full-duplex relaying } \\ \text { i.i.d. } & \text { Independent and identically distributed } \\ \text { INR } & \text { Self-interference-to-noise power ratio } \\ \text { IoT } & \text { Internet of Things } \\ \text { HD } & \text { Half-duplex relaying } \\ \text { KKT } & \text { Karush-Kuhn-Tucker } \\ \text { LMGF } & \text { Log moment generating function } \\ \text { LTE } & \text { Long-term evolution } \\ & \end{array}$




$\begin{array}{ll}\text { M2M } & \text { Machine-to-machine } \\ \text { MDP } & \text { Markov decision process } \\ \text { MIMO } & \text { Multiple-input multiple-output } \\ \text { N-FD-APA } & \text { Non-buffer full-duplex relaying with adaptive power allocation } \\ \text { N-FD-SPA } & \text { Non-buffer full-duplex relaying with static power allocation } \\ \text { pdf } & \text { Probability distribution function } \\ \text { QoS } & \text { Quality-of-service } \\ \text { RVIA } & \text { Relative value iterative algorithm } \\ \text { SI } & \text { Self-interference } \\ \text { SINR } & \text { Signal-to-interference-plus-noise power ratio } \\ \text { SNR } & \text { Signal-to-noise power ratio }\end{array}$




\section{List of Notations}

- $\mathbb{E}\{\cdot\}$ denotes the mathematical expectation.

- $\log (\cdot)$ represents the natural logarithm.

- $\log _{2}(\cdot)$ represents the logarithm to the base 2 .

- $\mathbb{R}^{+}$denotes the set of non-negative numbers.

- $\operatorname{Pr}(A)$ denotes the probability of event $A$.

- $\Omega(\theta)$ denotes log moment generating function of a random process.

- $\beta$ denotes self-interference cancellation parameter.

- $\mu$ denotes constant arrival rate to the source transmission buffer.

- $\zeta_{Q}$ denotes queue-length-outage probability.

- $Q$, and $Q^{\max }$ denote queue length random variable, and queue length bound, respectively.

- $\mathcal{H}$, and $\mathcal{Q}$ denote fading channel, and queue state spaces, respectively.

- $h[t] \in \mathcal{H}, P[t]$, and $r[t]$ denote channel power gain, power, and transmission rate, respectively, in frame $t$.

- $q[t] \in \mathcal{Q}$ denotes queue length in frame $t$.

- $p_{\mathcal{A}}(\cdot)$ denotes probability distribution function over some state space $\mathcal{A}$.

- $J(\cdot)$ (or $V(\cdot))$ denotes (pre-decision) state-value function. 
- $J_{\text {post }}(\cdot)$ (or $\left.V_{\text {post }}(\cdot)\right)$ denotes post-decision state-value function.

- $T$ denotes transmission frame duration.

- $B$ denotes channel bandwidth. 


\section{Chapter 1}

\section{Introduction}

\subsection{Motivation}

The past decade has seen the tremendous growth of wireless communications with the increasing demand for various emerging applications such as video transmissions, mobile entertainment, mobile healthcare etc., which require higher data rate and/or more stringent delay quality-of-service (QoS). Consequently, in the development of next-generation wireless systems, it is a crucial task to provide wireless connections with better QoS such as higher data rate, smaller delay etc. [1], [2]. However, such task is not easy due to many inherent challenges. One challenge is the fact that wireless signal strength randomly fluctuates over time due to varying fading [3]. There are large-scale fading effects, where the received signal strength changes over distance because of the path loss and shadowing, and small-scale fading effects, where the received signal strength changes because of the constructive and destructive interference of multiple reflecting and refracting signal paths. In addition, the available radio resources are limited. Hence, efficient (radio) resource allocation is crucial to combat the fading effects of wireless channels, and providing satisfactory QoS to the users [4].

On the communications over fading channels with data arrivals at the transmitter, due to the instantaneous transmission rate fluctuation over time, the arriving data might not be transmitted to the receiver instantly without delay. To cope with this situation, the transmitter uses a buffer to store the arriving data temporarily. Such data buffering enables the transmitter to exploit the temporal fading diversity by transmitting more data under more favorable channel conditions, which can result in power savings. With data buffering and appropriate power allocation, fading indeed becomes a benefit rather than an obstacle 
for wireless communications. On the other hand, data buffering incurs unavoidable queuing delay, which needs to be taken into consideration when developing resource allocation schemes [5]- [7]. It is well-known that providing delay QoS guarantees through efficient resource allocation is critical to the successful deployment of future wireless systems, which are expected to support various types of delay-sensitive applications such as real-time multimedia streaming, online games, video conferencing, intelligent transport systems etc [1], [2], [8]. In these applications, latency is the key QoS metric, and information has to be communicated from the transmitter to the receiver subject to different forms of delay constraints. Existing literature has considered two popular delay constraint models, namely average delay and delay-outage constraint models, each of which is suitable for a particular set of wireless applications in practice [7], [9], [10].

One of the main concerns when deploying wireless systems is the energy consumption. In traditional wireless communications systems, the devices have access to a fixed power supply, or are powered by replaceable/rechargeable batteries. In these cases, data transmissions are limited by power constraints of the battery-powered nodes to lengthen the system operational life. However, in many scenarios, a fixed power supply is not available, and even periodical battery replacement may not be a feasible option, for example, in large wireless sensor networks etc. In such situations, the use of energy harvesting (EH) for wireless communications appears appealing, or even essential by converting mechanical vibration into energy [11], by using solar panels [12], by utilizing thermoeletric generators [13], or by converting ambient radio power into energy [14]. EH provides a potentially infinite network operating time. Additionally, EH can help to reduce green-house gas emission level and cut down mounting energy cost for cellular service providers as compared to traditional energy sources such as fossil fuels. Due to its many advantages, EH technology is tempting in fifth-generation $(5 \mathrm{G})$ communications systems, where the wireless nodes are envisaged to be not only energy-efficient and but also self-sustainable [15]. Numerous enabling applications of EH can be found in the recently emerging Internet of Things (IoT) such as machine-tomachine (M2M) type communications [16], remote sensing, smart homes, smart cities [17], tactical networks [18] etc. To take full advantage of the EH technology, there has been a growing interest to develop resource allocation schemes for EH communication systems under various practical conditions [15], [19]- [21].

Motivated by the above discussions, this thesis studies novel resource allocation schemes for practical point-to-point and relaying communications systems to support delay-sensitive 
communications. In the considered systems, to overcome fading nature of wireless channels, the nodes (source and relay) use buffers to store the data arrivals, optimally allocate resources, and adapt transmissions to the instantaneous channel conditions to enhance the throughput. We also consider the case when the source is equipped with an EH module.

In the following, we briefly sketch the existing resource allocation designs under delay constraint.

\subsection{Resource Allocation under Delay Constraints}

Consider a point-to-point communication system over a fading channel with data arriving to the source transmission buffer. Due to fading, the amount of information being transmitted to the destination is random, which leads to random queuing delay for the buffered packets. Since it is hard to characterize the delay distribution, resource allocation to provide delay QoS guarantees is not trivial. In spite of that, there have been much progresses in this research area, for example, see [22] and references therein.

One well-known approach for delay QoS guarantees is to impose average delay constraint [9], [23]. Average delay constraint model can be applicable to some wireless applications such as file downloading, emails, and web browsing etc. which may require an average throughput under certain average delay. Power allocation under average delay constraint has been extensively studied, for example, see [22] and references therein. Several allocation algorithms with different complexities and performances have been developed using tools and results in large deviation theory [24], Lyapunov optimization theory [25], and Markov decision process (MDP) and stochastic control theory [26], [27]. The former two approaches, although simple, perform well under large delay regime only. On the other hand, the latter approach with higher complexity is optimal under all delay regimes.

In general, average delay constraint model is suitable for wireless applications, which do not require a specific bounded delay. However, for most emerging delay-sensitive applications such as real-time multimedia streaming etc., the key delay QoS requirement is the bounded delay. In other words, packets have to be delivered at the destination within a delay bound to be useful. Satisfying the average delay requirements does not necessarily satisfy the bounded delay requirements because the actual delay can differ much from the average delay. For instance, in case of call admission control of IEEE 802.11 standard, the distributed coordinated function results in a delay, which may be very different from its av- 
erage value [28]. The works [29], [30] consider power allocation problems for bounded delay constraints. Due to the random variations of the wireless fading channels with possible deep fades, providing bounded delay guarantees is either infeasible or results in a very high energy consumption or low transmission rate, in practice. For instance, the only lower bound of the transmission rate for bounded delay guarantees over a Rayleigh fading channel is zero [31].

Fortunately, most real-time multimedia applications can tolerate a certain small probability of delay bound violation. Hence, in order to support multimedia applications, delayoutage constraint can be employed, where the delay is allowed to exceed a delay bound within a maximum acceptable delay-outage probability [32], [33]. In general, the delay bound and delay-outage probability parameters are determined to satisfy the quality of experience (QoE) of the users. Note that when the delay-outage probability is close to 0, delay-outage constraint becomes bounded delay constraint. When the delay-outage probability is close to 1, it becomes unconstrained delay. Another advantage of delay-outage constraint is that it can relax the need for high power consumption, especially when the delay-outage probability is not too small because the source might not need to transmit under deep fades to save power. Using the delay-outage constraint model, a wide range of delay-sensitive applications can be modeled with different delay bounds and/or delay-outage probabilities. Assuming large delay regime assumption, and employing asymptotic delay analysis [34], the works [32], [33] have proposed the effective capacity (EC) concept, which is defined as the maximum supported constant arrival rate under statistical delay constraint. EC framework has been employed for performance analysis and resource allocation of many delay-sensitive communications systems [7], [10], [35]. Note that when the delay-outage probability converges to 1 , the EC becomes the ergodic capacity.

\subsection{Thesis Objectives, Contributions, and Outline}

We have seen that the central design challenge in future wireless systems is how to satisfy certain data rate and delay QoS requirements while making efficient use of available radio resources. The primary objective of this thesis is to develop novel resource allocation schemes to support delay-sensitive communications over fading channels for practical point-to-point and relaying communications systems, which have not been considered in existing literature. The specific issues, contributions, and corresponding outline can be elaborated as follows.

After Chapter 1, which presents the thesis motivation, objectives, and contributions, 
Chapter 2 reviews the most relevant works on resource allocation under delay constraints.

On the communications over fading channels, the works [9], [22], [23], [26], [27] have not considered the realistic case where only a portion of the random data arrivals can be buffered (or admitted) for transmissions. One scenario requiring admission control is to ensure queue stability (finite queue length) when there is insufficient power to stabilize the queue if all arriving data were to be buffered. In general, the admission control goal is to ensure as much arriving data as possible buffered for transmission without violating the delay (or queue stability) and power constraints. In Chapter 3, we address the optimal joint data admission control and power allocation (AC-PA) problem for throughput maximization under average delay and power constraints. To incorporate the randomness of channel fading, data arrival processes, as well as the constraints, we formulate the AC-PA problem as an infinite horizon constrained MDP [36]. Then, we propose a novel solution approach based on the so-called post-decision state-value function, which is used to rewrite the Bellman's optimality dynamic programming equation. The proposed approach requires smaller complexity than the traditional (pre-decision) state-value function approach since it does not include the channel states as its argument. Using the proposed approach, we can conveniently study the monotonicity and convexity properties of the optimal AC-PA solution with respect to the data arrival, channel fading, and queue length states. Numerical results for different delay and power constraints are compared and analyzed.

We next look at optimization for EH communications systems over fading channels, where random amounts of energy are harvested and stored in a battery by the transmitter during the course of data transmission from renewable energy sources [37]- [39]. While these existing contributions do not consider delay constraints, Chapter 4 presents our findings on optimal power allocation for EH systems for source arrival rate maximization under average delay or delay-outage constraint. In the latter case, we convert the original problem into an EC maximization problem. By adapting the approach in Chapter 3, the resulting problems are solved using MDP and post-decision state-value function approach. The proposed approach imposes causality constraint on the use of the harvested energy, where the transmitter is constrained to use at most the amount of stored energy currently available in every time slot, although more energy may become available in the future. The optimal solutions take into account the random variations in amounts of harvested energy, and channel conditions to compute the power allocation in each time slot. Moreover, in the case of delay-outage constraint, the power allocation algorithm does not need to keep track of the data queue 
length since large delay regime is assumed. Hence, the algorithm requires less complexity as compared to the case of average delay constraint. The monotonicity of the optimal solutions is studied, revealing valuable insights into how to optimally allocate the power with respect to the channel fading, battery, and data queue length states. It is illustrated that the proposed approach achieves higher data rates than existing heuristic approaches. We demonstrate the different effects of the two delay constraint models on the system performance in terms of supported rates and delay performance.

In general, the pre-decision state-value functions (as well as the optimal solutions) of the MDP problems considered in Chapters 3, and 4 can be computed using relative value iteration algorithm (RVIA) or dynamic programming when the statistical knowledge of the underlying random (e.g., channel fading, data arrival, EH) processes is known [40]. When such knowledge is unavailable, which is typical in real-life communications, we also propose online allocation algorithms, which update the state-value functions as new samples of the random processes are realized during transmissions. The proposed algorithms provide less complexity, and faster convergence than the conventional reinforcement learning algorithms, which learn the pre-decision state-value functions instead [41]. From the results in stochastic approximation theory, the proposed algorithms converge to the optimal solutions for all channel models (e.g., discrete or continuous channel fading states) [42].

In Chapters 3, and 4, we have considered point-to-point communications, which is not always possible in practice, for example, due to long distance, or severe shadowing. In such scenarios, wireless relaying provides an efficient means to improve the coverage, throughput, and reliability of wireless networks. Consider a simple 3-node source-relay-destination relaying system. Recently, the works [43], [44] introduce the idea of buffer-aided relaying, where the relay employs buffer to store the received data from the source for future transmission to the destination. Thanks to the buffer-aided relaying capability, adaptive link selection relaying is possible, where either the source-relay link or the relay-destination link is active depending on the channel state information (CSI) in each transmission frame [45]- [47]. While adaptive relaying is able to exploit the link fading diversity, one disadvantage is the (queueing) delay incurred at the relay buffer, which is assumed to be unconstrained or under average delay constraint in existing works [43]- [47]. Alternatively, in Chapter 5, we study the optimal adaptive relaying problem under delay-outage constraint. Both cases of fixed and adaptive power allocation are considered. In general, the delay-outage constraint is intractable since we need to know the tail distributions of the source and relay queue 
lengths. To overcome the difficulty, we employ asymptotic delay analysis to transform the delay-outage constraint into constraints on the minimum exponential decay rates (or delay exponents) of tail distributions. The relationships between the delay exponents and resource allocation variables are then derived. Consequently, we obtain tractable constrained optimization problems. We then derive the optimal solutions as functions of the instantaneous CSI and delay exponents (or equivalently, delay-outage constraint) using Lagrangian approach and convex optimization. Moreover, based on the derived optimal solutions, impacts of the delay constraint on the resource allocation solutions are studied. Specifically, the power allocation solution is shown to converge to the conventional water-filling and channelinversion policies under very loose and stringent delay constraints, respectively. In general, the power allocation solution swings between the two policies. In addition, we show that, under very loose delay constraints, the allocation solutions converge to the solutions derived in [46] under unconstrained delay assumption. Illustrative results show that the proposed adaptive relaying outperforms fixed relaying under sufficiently loose delay constraints.

Last, under adaptive relaying, the relay can either receive data from the source or transmit data to the destination. Such half-duplex (HD) relaying avoids self-interference (SI) at the expense of low spectral efficiency. Moreover, recently-developed SI mitigation methods can leverage the potential of full-duplex (FD) relaying in which a relay can receive and transmit simultaneously over the same frequency band [48]- [50]. However, SI still cannot be completely mitigated in practical systems, and the resulting non-zero residual SI reduces the performance of FD relaying. Hence, in order to evaluate the potential benefits of FD relaying over HD relaying, such non-zero residual SI needs to be taken into account. Chapter 6 addresses the power allocation problems for buffer-aided FD relaying with imperfect SI cancellation under delay-outage constraint. The non-zero residual SI is assumed to be zero-mean, additive and Gaussian with the variance proportional to the relay transmit power as commonly assumed in existing literature [51]- [54]. We investigate two power allocation problems: (i) Buffer-aided FD relaying with adaptive power allocation when the instantaneous CSI is available at the transmitters (CSIT); (ii) Buffer-aided FD relaying with static power allocation when only statistical CSIT is available. While instantaneous CSIT may be unavailable due to high signaling complexity for CSI feedback from the receivers, statistical CSIT can always be accessible, since the duration over which channel fading processes are stationary is several orders of magnitude longer than the duration of the fades. The optimal solutions are derived using asymptotic delay analysis. Solutions for special cases of statisti- 
cal delay constraint and residual SI are studied. Specifically, it is shown with instantaneous CSIT, FD relaying approaches HD relaying with adaptive link selection studied in Chapter 5 when the residual SI becomes very large. Consequently, FD relaying always outperforms HD relaying since the former can dynamically switch between HD/FD operation modes depending on the instantaneous channel conditions. On the other hand, with statistical CSIT, FD relaying outperforms HD relaying under good SI cancellation only. Also, buffer-aided FD relaying is more beneficial than non-buffer FD relaying.

Finally, Chapter 7 concludes the thesis and discusses some future research directions. 


\section{Chapter 2}

\section{Power Allocation over Fading Channels under Delay Constraints: A Literature Review}

In many wireless communication scenarios, energy management is an important issue for reasons such as extending a device's usable life-time. Since transmission power is one of the main energy consumers in wireless devices, efficient power allocation has been an important challenge, which has attracted significant research interests. Consider a point-to-point communications link over a fading channel with random data arrivals at the source. Due to fading, the channel conditions (and the corresponding instantaneous transmission rates) unpredictably fluctuate over time. Hence, the arriving data might not be transmitted to the destination instantly without delay. To overcome the fading nature of wireless channels, the source uses a buffer to store the data arrivals temporarily, which introduces random queuing delay as a consequence. Intuitively, for power savings, the source can simply defer the packet transmission during 'bad' channel states, and transmit more packets during 'good' channel states, i.e., more power is allocated under more favorable channel conditions. However, such transmission mechanism can lead to long delays for buffered packets since 'bad' channel states can happen often. As a result, delay QoS guarantees cannot be provided as required in order to support delay-sensitive communications.

Toward this end, several power allocation schemes over fading channels have been proposed to support delay QoS guarantees as briefly discussed in Chapter I: Introduction. In 


\section{Power Allocation over Fading Channels under Delay Constraints: A Literature Review}

this chapter, we will discuss this topic in greater detail, with comments on materials related to our research subjects as follows. In Sections 2.1 and 2.2, power allocation schemes for a source-destination communications link under average delay, and delay-outage constraints, respectively are presented. Section 2.3 reviews existing contributions on power allocation with energy harvesting. In Section 2.4, resource allocation schemes for buffer-aided relaying communications are described, which include both cases of half-duplex and full-duplex relaying.

\subsection{Average Delay Constraint}

For delay QoS guarantees, one possible power allocation goal is to minimize the (average) power under a constraint on the (maximum) average delay. Depending on the delay constraint, transmissions can take place even under unfavorable channel conditions since the power allocation is based not only on the channel conditions but also on the current delay of the buffered data. Such design problem has been addressed in many works, for example, see [9], [23], [25]- [27], [55]- [59] and references therein. The central concept is the optimal power- delay trade-off, i.e., the minimum power required to attain a delay bound [23]. As the delay bound increases implying looser delay constraints, less power is needed since the source can delay transmissions until more favorable channel conditions happening to save power. The structural results of the policies achieving the optimal trade-off (or optimal policies) have been studied in [23], [27], [58]. In general, it is proved that the optimal power allocation increases as the queue length increases, and decreases as the channel state goes from good to bad. It means that the optimal decision is to transmit a certain amount of data at any given instant, where this amount increases with the current queue length and decreases with the channel state. Thus for a fixed channel gain, the greater the queue length the more you transmit, and for a fixed queue length, the better the channel, the more you transmit. Such transmission mechanism, intuitively, can help to reduce the delay and save power simultaneously.

There are several approaches with different complexities and performances to develop power allocation algorithms under average delay constraint, for example, see [22] and references therein. The proposed approaches rely on tools and results in large deviation theory [24], Lyapunov optimization theory [25], or Markov decision process (MDP) and stochastic control theory [23], [26], [27], [57], [58]. While the former two approaches allow potentially 


\section{Power Allocation over Fading Channels under Delay Constraints: A Literature Review}

simple solutions depending on the channel state information (CSI) only, the resulting policies perform well only for the large delay regime, i.e., asymptotically optimal, where the transmission buffers are assumed to be non-empty. This is because the dynamics of the queue length (or buffer) is not considered when allocating the transmit power. On the other hand, the MDP-based approach achieves optimal performance for all delay regimes at the expense of higher control complexity since it needs to take into account both the CSI and the queue length state, as well as their dynamics when calculating the allocated power. It incorporates the randomness of the channel fading and data arrival processes in the optimal solutions. When the statistical knowledge of the random channel fading and data arrival processes is known, optimal power allocation policies as solutions of the MDP problems can be computed off-line, for instance by using dynamic programming techniques [40]. However, such statistical knowledge is often unavailable in real-life communications, and hence, developing online allocation algorithms without requiring known statistics of the random processes is an important issue [26], [27], [59].

In [9], [23], [26], [27], it is shown that a given delay bound can be attained by allocating a sufficient amount of transmit power. In Chapter 3, we consider a practical scenario where the source is assumed to have a maximum power constraint, which is insufficient to attain the given delay bound. In this case, admission control needs to be applied on random data arrivals to the source buffer to avoid (delay and power) constraint violation. The goal of admission control (jointly with power allocation) is to maximize the average admitted rate, i.e., throughput maximization. In [25], the author proposes the energy constrained control algorithm (ECCA) for joint admission control and power allocation (AC-PA) using Lyapunov optimization theory. While ECCA cannot achieve optimal outcomes, Chapter 3 studies the optimal AC-PA problem using MDP and stochastic control tools. Unlike the ECCA, the proposed AC-PA algorithm incorporates the dynamics of the buffer as well as the random variations of the channel fading, and data arrivals when computing the admission control and power allocation solution in each transmission time slot. Hence, the proposed algorithm provides higher throughput than ECCA under similar delay and power constraints.

\subsection{Delay-Outage Constraint}

In the above-mentioned works, the resource allocation designs are to provide average delay bound guarantees, which are suitable for applications such as email, file downloading, 


\section{Power Allocation over Fading Channels under Delay Constraints: A Literature Review}

etc. These applications do not require a specific bounded delay, which is the case for most other delay-sensitive applications such as real-time multimedia streaming, video conference etc. Moreover, it is clear that average delay bound satisfaction do not necessarily guarantee bounded delay requirement. Moreover, due to the random variations of the wireless fading channels with possible deep fades, providing bounded delay guarantees is either infeasible or results in a very high energy consumption or low transmission rate. Fortunately, most real-time multimedia applications can tolerate a certain small probability of delay bound violation. Hence, to support real-time multimedia applications, delay-outage constraint can be employed, where the delay is allowed to exceed a delay bound within a maximum acceptable delay-outage probability [32], [33]. In particular, on the communications over fading channels as described above, we are interested in resource (or power) allocation to maximize the supportable constant data arrival rate to the source transmission buffer under given delay-outage constraint.

To handle the delay-outage constraint, we need to know the (tail) distribution of the delay, which is difficult to derive in general for given arrival and service (or capacity) processes. However, if large delay regime is assumed, we can then employ the asymptotic delay analysis to characterize the tail distribution of the delay using an exponentially decreasing function [34], [60].

\subsubsection{Asymptotic Delay Analysis}

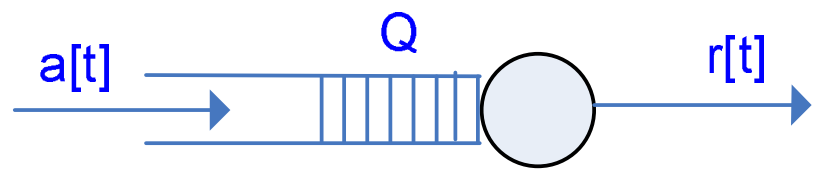

Fig. 2.1: Dynamic queue with arrival $\{a[t]\}$ and service $\{r[t]\}$ processes.

Consider a time-slotted stable queue with infinite buffer size as in Fig. 2.1. Consider stationary and ergodic arrival process $\{a[t]\}$ and service process $\{r[t]\}$ with the domain, range, and unit being $[0, \infty), t=1,2, \ldots$, and bits per time-slot, respectively. The processes are assumed to satisfy the Gartner-Ellis limit [60], i.e., for all $\theta \geq 0$, their differential 
asymptotic logarithmic moment generating functions (LMGFs) $\Omega_{a}(\theta)$ and $\Omega_{r}(\theta)$ defined as:

$$
\Omega_{a}(\theta)=\lim _{t \rightarrow \infty} \frac{1}{t} \log \mathbb{E}\left\{e^{\theta \sum_{\tau=1}^{t} a[\tau]}\right\} ; \quad \Omega_{r}(\theta)=\lim _{t \rightarrow \infty} \frac{1}{t} \log \mathbb{E}\left\{e^{\theta \sum_{\tau=1}^{t} r[\tau]}\right\}
$$

exist, where $\mathbb{E}\{\cdot\}$ denotes mathematical expectation operator. Note that for i.i.d. processes $\{a[t]\}$, and $\{r[t]\}$, we have:

$$
\Omega_{a}(\theta)=\log \mathbb{E}\left\{e^{\theta a[t]}\right\}, \quad \Omega_{r}(\theta)=\log \mathbb{E}\left\{e^{\theta r[t]}\right\}
$$

Assume independent processes with $\mathbb{E}\{a[t]\}<\mathbb{E}\{c[t]\}$. If there exists an unique delay exponent $\bar{\theta}>0$ satisfying the following equation:

$$
\Omega_{a}(\bar{\theta})+\Omega_{r}(-\bar{\theta})=0
$$

then, for sufficiently large $x$, the tail distribution the steady-state queue-length random variable $Q$ is given as follows [60, Theorem 2.1]:

$$
\operatorname{Pr}(Q>x)=e^{-\bar{\theta} x}
$$

where $\operatorname{Pr}(Q>x)$ denotes the probability of the event $Q>x$. The rigorous proof based on large deviations principles is presented in [34], and is omitted for brevity. We can see that, under large queue length (or delay) regime, the tail distribution function of the queue length is an exponentially decreasing function with decay rate $\bar{\theta}$. A smaller $\bar{\theta}$ corresponds to a slower decay rate, while a larger $\bar{\theta}$ leads to a faster decay rate.

\subsubsection{Effective Capacity}

Consider the queue in Fig. 2.1 with constant data arrival process $\{a[t]=\mu\}$ with LMGF $\Omega_{a}(\theta)=\mu \theta$ instead and some service process $\{r[t]\}$ with LMGF $\Omega_{r}(\theta)$. Suppose that we impose the following delay-outage constraint in terms of the maximum queue-length-outage probability constraint:

$$
\operatorname{Pr}\left(Q>Q^{\max }\right) \leq \zeta_{Q}
$$




\section{Power Allocation over Fading Channels under Delay Constraints: A Literature Review}

for given queue length bound $Q^{\max } \in(0, \infty)$ and queue-length-outage probability $\zeta_{Q} \in(0,1]$. The constraint on a small $\zeta_{Q}$ is applicable to delay QoS requirements, in which the user applications are acceptable as long as the queue length (or delay) does not exceed a threshold $Q^{\max }$, and $\zeta_{Q}$ indicates how stringent the delay constraint is. For a given $Q^{\max }$, smaller $\zeta_{Q}$ indicates more stringent delay constraints. When $\zeta_{Q}$ is close to 0 , the queue length cannot exceed $Q^{\max }$, i.e., (deterministic) bounded delay constraint. When $\zeta_{Q}$ is close to 1 , we allow unconstrained queue length.

Assume $Q^{\text {max }}$ sufficiently large (but finite) so that the asymptotic delay analysis result (2.3) can be applied. From (2.2) and (2.3), we can see that, in order to meet the constraint (2.4), the arrival rate $\mu$ has to satisfy the following conditions:

$$
\mu \bar{\theta}+\Omega_{r}(-\bar{\theta})=0 ; \quad \bar{\theta} \geq \theta^{\operatorname{tar}} \triangleq-\log \left(\zeta_{Q}\right) / Q^{\max }
$$

for some delay exponent $\bar{\theta}>0$. This is because from $(2.3)$, we would have: $\operatorname{Pr}\left(Q>Q^{\max }\right)=$ $e^{-\bar{\theta} Q^{\max }} \leq \zeta_{Q}$ as required. Then, it can be seen that the maximum supportable arrival rate $\mu^{\max }$ satisfying $(2.5)$ is achieved when $\bar{\theta}=\theta^{\operatorname{tar}}$, and is given by:

$$
\mu^{\max }=-\frac{\Omega_{r}\left(-\theta^{\operatorname{tar}}\right)}{\theta^{\operatorname{tar}}}
$$

$\mu^{\max }$ is called the effective capacity (EC) of the service process $\{r[t]\}$ with delay exponent $\theta^{\text {tar }}$, which is derived from the delay-outage constraint (2.4).

We shall call the function $-\Omega_{r}(-\theta) / \theta$ the EC function of the service process $\{r[t]\}$ (with delay exponent $\theta)$.

\subsubsection{EC-based resource allocation and performance analysis}

Delay-outage constraint model and EC framework have been employed to analyze the performance and develop many resource allocation schemes for various wireless communications systems. This is because it is particularly convenient for analyzing the delay-outage performance of wireless transmissions where the service process $\{r[t]\}$ is determined by the instantaneous capacity of the wireless fading channel.

As an example, consider power allocation for EC maximization for point-to-point communications system over fading channel with bandwidth $B(\mathrm{~Hz})$ [61]. We assume ergodic stationary independent and identically distributed (i.i.d.) block-fading channel with fading 


\section{Power Allocation over Fading Channels under Delay Constraints: A Literature Review}

duration $T$ (seconds) equal to the transmission frame, i.e., the channel power gains remain unchanged during a frame but vary independently from frame to frame. Denote $h[t]$, and $P[t]$ the instantaneous (normalized) channel gain, and transmit power, respectively, in frame $t=1,2, \ldots$. Let $r[t]$ denote the corresponding instantaneous transmission rate (or capacity) in frame $t$, which is given by the Shannon's formula:

$$
r[t]=\log _{2}(1+P[t] h[t]) .
$$

From (2.6), the optimal power allocation problem to maximize the effective capacity (with delay exponent $\theta$ ) under maximum average power constraint can be formulated as:

$$
\max _{P[t] \geq 0}-\frac{1}{\theta T B} \log \mathbb{E}\left\{e^{-\theta T B r[t]}\right\} \quad \text { s.t. : } \quad \mathbb{E}\left\{P^{*}[t]\right\} \leq \bar{P}^{\max }
$$

where $\bar{P}^{\text {max }}$ is the maximum (average) power. Using Lagrangian approach, after some simple manipulations, the optimal power allocation can be shown to be:

$$
P^{*}[t]= \begin{cases}\left(\frac{\hat{\theta}}{\lambda(h[t])^{\theta}}\right)^{\frac{1}{1+\hat{\theta}}}-\frac{1}{h[t]}, & h[t] \geq \frac{\lambda}{\hat{\theta}} \\ 0, & \text { otherwise }\end{cases}
$$

where we denote (normalized) delay exponent $\hat{\theta}=\theta T B / \log (2)$, and $\lambda$ is the Lagrange multiplier satisfying the following condition:

$$
\mathbb{E}\left\{P^{*}[t]\right\}=\bar{P}^{\max }
$$

Alternatively, we can consider the power minimization problem subject to the minimum EC constraint. In [62]- [64], the authors study the power allocation problems for EC or energy efficiency maximization for multi-channel settings, i.e., orthogonal frequency division multiplexing (OFDM). We omit the details here for brevity.

EC framework has also been considered in many other communications scenarios. For example, the effective capacities of multiple-input multiple-output (MIMO) antenna systems, and multiple access channels are analyzed in [65], and [66], respectively. In [67], [68], the authors studied scheduling policies for multi-user cellular networks. In [69], [70], the authors 


\section{Power Allocation over Fading Channels under Delay Constraints: A Literature Review}

consider sub-channel and power allocation for power minimization for multi-user OFDM systems under minimum effective capacity constraints of the users. In [71], the authors propose an framework to jointly optimize effective spectrum efficiency and effective power efficiency under different delay-outage constraints.

In Chapters 4, 5, and 6, we will employ the delay-outage constraint and EC notion as criteria to develop resource allocation schemes for two communications systems: 1) A source-destination communications link with energy harvesting; 2) A 3-node source-relaydestination buffer-aided relaying system, where the buffers are employed at both the source and relay. In this case, the delay-outage constraint is imposed on the end-to-end delay, which is the sum of delays at the source and relay buffers.

\subsection{Energy Harvesting Communications Systems}

We have seen that future wireless communications systems are expected to accommodate an ever increasing number of wireless applications with high capacity demands and/or stringent QoS requirements such as real-time multimedia streaming, connected and autonomous vehicles etc. [1]. Moreover, supporting higher data rates under strict delay QoS requirements increases the energy consumption, which results in a detrimental impact on the environment. A challenge for future wireless system design is to meet the increasing energy demand, while lowering the emission of greenhouse gases for achieving the environment sustainability. Consequently, green communications has attracted significant attention in academia and industry. An efficient and promising technology to tackle this issue is energy harvesting (EH), where wireless EH nodes harvest energy from the renewable sources of their surrounding environment, convert it to electrical energy, and use the electrical energy in order to carry out their functions. In addition to greenhouse gas emission reduction, EH technology is also appealing for communications scenarios when a fixed power supply is not available, and even periodical battery replacement may not be a feasible option for communications devices, for example, in large wireless sensor networks etc. In such cases, EH provides a way of operating the network with a potentially infinite lifetime. EH nodes are particularly suitable for machine-to-machine (M2M), and Internet-of-Things (IoT) communication systems etc. as they are envisaged to be both energy-efficient and self-sustainable [16], [17].

There has been a growing interest in the optimization of $\mathrm{EH}$ communication systems, which has to address the challenging issue of instability of renewable energy resources. In 


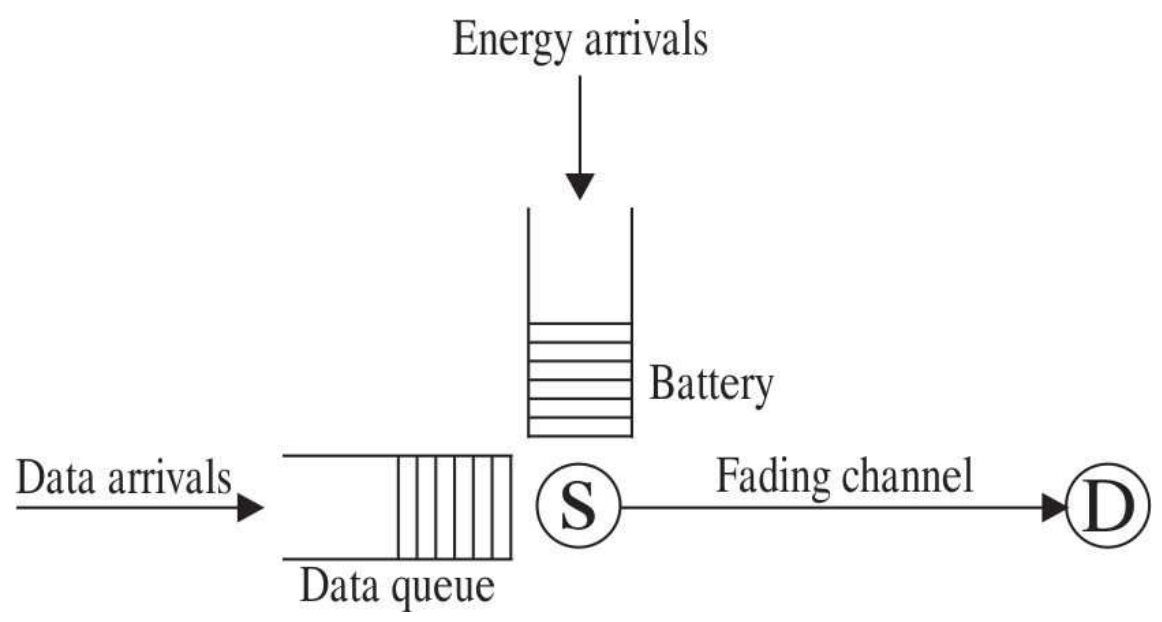

Fig. 2.2: A source-destination communications link with EH transmitter.

particular, power allocation issues for EH communication systems have been investigated [15], [20], [21], [37]- [39]. Unlike the case of fixed power supply, power allocation for EH transmitters is subject to $\mathrm{EH}$ constraints, where in every time slot, each transmitter is constrained to use at most the amount of stored energy currently available, although more energy may become available in the future slots. Consider EH communications systems over fading channels, where the random energy arrivals are stored in battery for data transmission as in Fig. 2.2. In [37], the authors study the throughput maximization problem assuming delay-limited communications, where a randomly arriving packet at the source is decided to be either transmitted or dropped without buffering. A learning theoretic approach is introduced, which does not require any statistical information on the random fading channel, energy arrival, and data arrival processes. The works [38], [39] explore various throughput maximization problems assuming data arrivals being stored in a data buffer. However, it is noted that these works do not consider delay constraints. In [72], [73], power allocation schemes for EH systems are proposed to ensure the stability of the data and energy queues (or battery) using Lyapunov optimization theory. In [74], the authors derived the EC for EH systems for given power allocation policies. The derived expressions were then exploited to evaluate commonly used power allocation policies, e.g., greedy policy, constant power policy, etc. assuming the statistical knowledge about the random processes is known.

In Chapter 4, consider the EH system as in Fig. 2.2, we explore optimal stochastic 
power allocation problems for such EH system over fading channels under average delay or delay-outage constraints. We develop online power allocation algorithms when the statistical knowledge of the random channel fading, EH processes is unknown, which is typical in reallife communications. The studies provide valuable insights into how to optimally allocate power under different types of delay constraints.

\subsection{Buffer-Aided Relaying Communications}

The above-mentioned works concern resource allocation for point-to-point communications. In practice, it is not always possible for a source to communicate directly with the destination, for example, due to long distance, or severe shadowing. An example is downlink communications from the base station to the cell-edge users. In such scenarios, wireless relaying provides an efficient means to improve the coverage, throughput, and reliability of wireless networks. Typical situations where wireless relaying is needed are depicted in Fig. 2.3 .

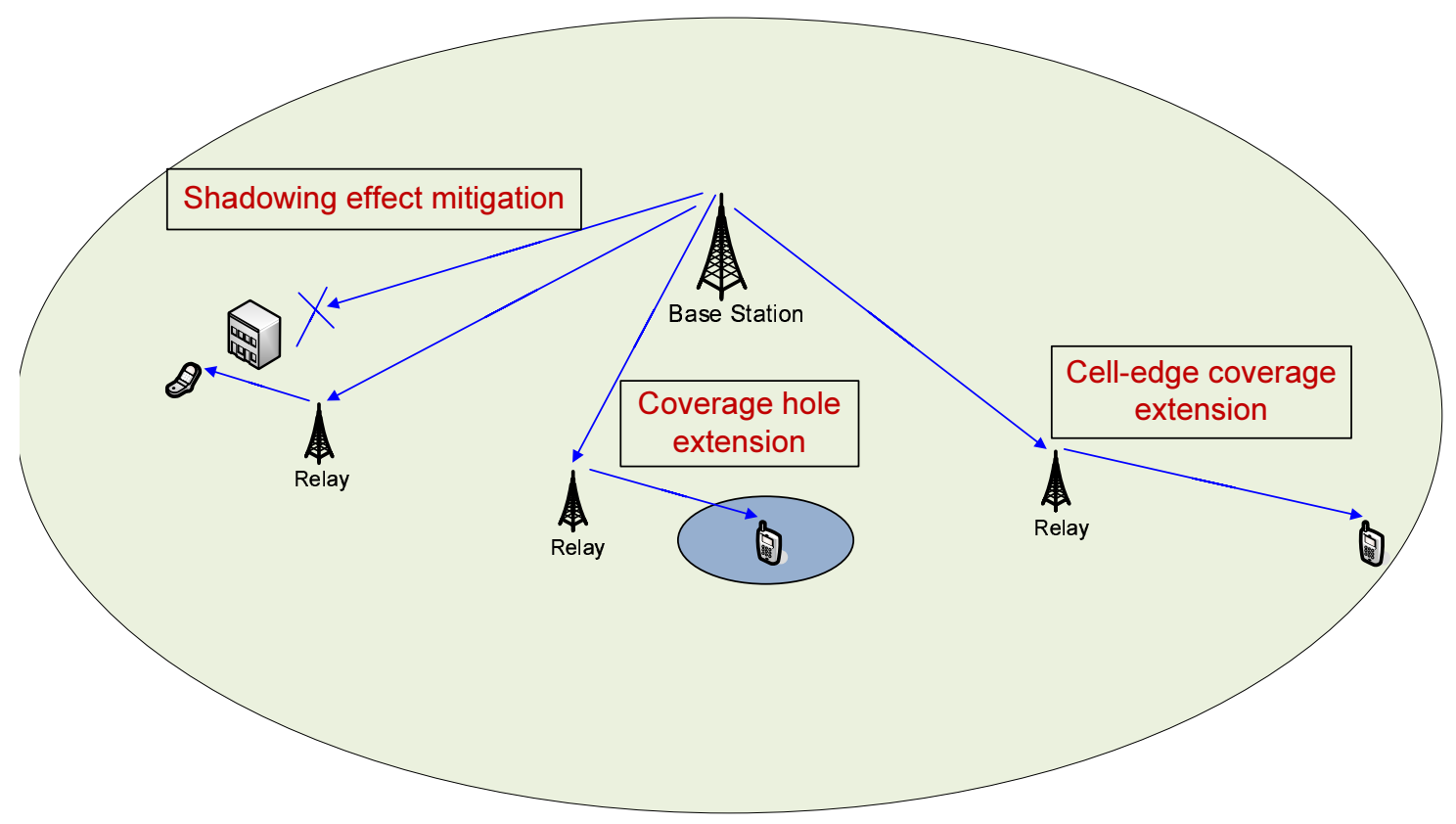

Fig. 2.3: Relaying in wireless cellular communications.

Relaying has been adopted by recent wireless communications standards, e.g., 3GPP- 


\section{Power Allocation over Fading Channels under Delay Constraints: A Literature Review}

Long Term Evolution (LTE) [75]. There has been a great deal of research on the 3-node relay network over the past decades under different configurations, (e.g., with or without direct source-destination link) and relaying schemes, (e.g., decode-and-forward or amplifyand-forward relaying), for example, see [76], and references therein. In these works, the relay receives packets from the source in one time slot, and forwards it to the destination in the next time slot, which is referred to as fixed relaying (or fixed link scheduling) in the sequel. Such fixed relaying schemes may suffer significant performance degradation over fading channels, where the source-relay (S-R) or relay-destination (R-D) link signal strengths can greatly vary with time since the end-to-end transmission rate is dominated by the weaker link of the two links. For example, for a 3-node decode-and-forward relaying network without a direct S-D link, the capacity is given by the minimum of the S-R and the R-D link capacities [77].

\subsubsection{Half-duplex Relaying with Adaptive Link Selection}

Recent works have introduced buffer-aided relaying, where the relay employs buffer to store the received data from the source for future forwarding to the destination [43], [44]. Under buffer-aided relaying, the relay has more transmission flexibility since it might not need to forward the received data to the destination immediately after receiving it as in the case of fixed relaying. Hence, buffer-aided relaying can overcome the fading effects of wireless channels. In general, fixed relaying schemes developed under non-buffer relaying setting can be modified to exploit the relay buffering. However, the resulting relaying schemes may fail to achieve the maximum diversity gain offered by buffer-aided relaying over the non-buffer relaying since the relay still receives and transmits sequentially in every time slot [43], [78]- [80]. Thus, to exploit the transmission flexibility offered by the relay buffering capability, adaptive link selection relaying must be considered, where the relay transmission and reception schedule is not fixed. Such adaptive relaying efficiently schedules the S-R link and R-D link depending on their channel conditions in each frame. As a result, adaptive relaying can attain significant throughput gains over fixed relaying since it can exploit the link diversity by transmitting over the link with more favorable channel condition [45]- [47].

One disadvantage of buffer-aided adaptive relaying is that it introduces random queuing delay at the relay, which is not present under non-buffer relaying. Most existing adaptive relaying schemes are developed under the unconstrained delay or average delay constraint settings which are reviewed in the following. 


\subsubsection{Case of Unconstrained Delay}

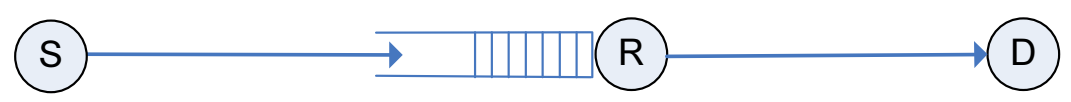

Fig. 2.4: Buffer-aided relaying model.

Consider 3-node buffer-aided relay network in Fig. 2.4. Assume the source always has data to transmit. We assume ergodic stationary i.i.d. block-fading channels with fading duration equal to the transmission frame. Denote $h_{1}[t]$, and $h_{2}[t]$ the instantaneous (normalized) channel gains in frame $t=1,2, \ldots$ of the S-R link and R-D link, respectively. $h_{i}[t], i=1,2$ are assumed to be statistically independent random variables. Let $P_{1}$ and $P_{2}$ denote the transmit powers of the source and relay, respectively. Similarly, denote $r_{i}[t], i=1,2$ the corresponding instantaneous transmission rates in frame $t=1,2, \ldots$ of the links:

$$
r_{i}[t]=\log _{2}\left(1+P_{i} h_{i}[t]\right), i=1,2 .
$$

In [46], the authors consider the adaptive link selection relaying problem described as follows. Let $\phi[t] \in\{0,1\}, \forall t$ denote a binary variable for frame $t$ where we set $\phi[t]=1$ if the R-D link is active and $\phi[t]=0$ if the S-R link is active. The adaptive relaying scheme for throughput maximization is shown to have the following form [46]:

$$
\phi[t]= \begin{cases}0, & r_{1}[t] / r_{2}[t] \geq \xi \\ 1, & \text { otherwise }\end{cases}
$$

where the parameter $\xi>0$ is determined to maintain the following equality:

$$
\mathbb{E}\left\{(1-\phi[t]) r_{1}[t]\right\}=\mathbb{E}\left\{\phi[t] r_{2}[t]\right\}
$$

Intuitively, the link scheduling solution ensure equal average arrival rate and departure rate of the relay buffer. We can see that the adaptive link scheduling exploits the link fading diversity by transmitting over a link when the ratio between its rate and rate of the other link is larger than a threshold value $\xi$. The threshold $\xi$ takes into account the fading statistics and average signal-to-noise power ratios (SNRs) of the S-R and R-D links. Then, the (average) 
throughput of the adaptive relaying scheme is:

$$
\mathcal{T}_{\mathrm{B}-\mathrm{ALS}}=\mathbb{E}\left\{(1-\phi[t]) r_{1}[t]\right\}
$$

To show the advantages of adaptive link selection relaying, consider the case that the links have the similar fading distributions with equal average SNRs as an example. The optimal $\xi$ in (2.8) can be easily seen to be 1, i.e., the link with larger instantaneous rate is selected in each slot. The throughput of adaptive link selection relaying can be shown to be:

$$
\mathcal{T}_{\mathrm{B}-\mathrm{ALS}}=\frac{1}{2} \mathbb{E}\left\{\max \left\{r_{1}[t], r_{2}[t]\right\}\right\} .
$$

Consider two non-buffer and buffer-aided relaying schemes with fixed link schedules. With non-buffer relaying, the relay receives a packet in one time slot and transmits it in the next, and the corresponding average throughput is [77]:

$$
\mathcal{T}_{\mathrm{N}-\mathrm{FLS}}=\frac{1}{2} \mathbb{E}\left\{\min \left\{r_{1}[t], r_{2}[t]\right\}\right\}
$$

With buffer-aided fixed relaying scheme, the relay receives data from the source in the first $N / 2$ ( $N$ is even) time slots and sends this cumulative information to the destination in the next $N / 2$ slots [43]. The corresponding maximum achievable throughput is obtained for $N \rightarrow \infty$ and given by:

$$
\mathcal{T}_{\mathrm{B}-\mathrm{FLS}}=\frac{1}{2} \min \left\{\mathbb{E}\left\{r_{1}[t]\right\}, \mathbb{E}\left\{r_{2}[t]\right\}\right\}
$$

We can see that it always holds true that:

$$
\mathcal{T}_{\mathrm{B}-\mathrm{ALS}}>\mathcal{T}_{\mathrm{B}-\mathrm{FLS}} \geq \mathcal{T}_{\mathrm{N}-\mathrm{FLS}}
$$

Note that adaptive power allocation in each slot can be considered in addition to the link selection [46].

The work [47] considers the similar buffer-aided relaying model as in [46], and studies two adaptive link scheduling schemes with different requirements regarding the availability of CSIT. In the first scheme, neither the source nor the relay has full CSIT, and consequently, both nodes are forced to transmit with fixed rates. On the other hand, in the second scheme, the source does not have full CSIT and transmits with fixed rate but the relay has full CSIT 


\section{Power Allocation over Fading Channels under Delay Constraints: A Literature Review}

and adapts its transmission rate accordingly. The optimal link scheduling solutions and the corresponding throughput are derived. We omit the details for brevity.

Buffer-aided adaptive relaying has been considered in other settings too. For example, in [81] - [83], the authors study the adaptive link scheduling schemes for throughput maximization for two-way relaying. Moreover, buffer-aided adaptive relaying is also employed in 3-hop relay network [84].

We can see that buffer-aided adaptive link selection relaying has significantly improved the performance of non-buffer relaying due to its capability to exploit the link fading diversity. However, we should emphasize that the QoS-blind adaptive relaying schemes in the aforementioned works introduce unconstrained (or infinite) relaying delay, i.e., the relaying delay can be very large [46], [47]. Hence, in order to support delay-sensitive communications, new adaptive relaying schemes have to be developed.

\subsubsection{Case of Average Delay Constraint}

There have been several attempts to develop buffer-aided adaptive relaying schemes to provide delay QoS guarantees. In particular, several relaying schemes have been developed by heuristically modifying the aforementioned QoS-blind relaying schemes to satisfy average delay constraint [46], [47], [83], [84]. The schemes take into account the instantaneous link conditions and amount of data in the relay buffer based on the observation that the (average) delay can be controlled via the arrival rate and the relay buffer size. Two different approaches to adjust the arrival rate and the queue size are proposed. One approach is to 'starve' the buffer by intentionally limiting the arrival rate by choosing a threshold which is strictly smaller than $\xi$ in (2.8). Another approach is to limit the buffer size by forcing the relay to transmit if the relay buffer gets full. We omit the details for brevity. Note that both proposed relaying schemes are heuristic in nature, i.e., sub-optimal schemes.

We have seen that the developed adaptive relaying schemes assume unconstrained delay or average delay constraint. Alternatively, in Chapter 5, we study optimal adaptive relaying scheme under (end-to-end) delay-outage constraint to maximize the EC, i.e., constant supportable arrival rate to the source buffer. Under the proposed design, the link selection solution depends not only on the link conditions as in the case of unconstrained delay but also on the delay constraint. To tackle the delay-outage constraint, we apply the asymptotic delay analysis in Section 2.2, to transform the delay-outage constraint into the constraints on 


\section{Power Allocation over Fading Channels under Delay Constraints: A Literature Review}

the minimum delay exponents at the source and relay buffers. We then derive the relationship between the link selection variables and the delay exponents, which is used to obtain tractable constrained optimization problem. The solution derived under delay-outage constraint is expected to converge to the solution (2.8) derived under unconstrained delay assumption when the delay-outage probability converges to 1 .

\subsubsection{Full-duplex Relaying}

Under adaptive relaying, the relay can either receive data from the source or transmit data to the destination. Such half-duplex (HD) relaying avoids self-interference (SI) at the expense of low spectral efficiency. Recently, several effective SI mitigation techniques have been developed, based on combinations of antenna, analog, and digital cancellations, e.g., [48][50]. Such results promise the potential full-duplex (FD) relaying operation, in which a relay can receive and transmit simultaneously to enhance the relay system spectral efficiency [85]. Earlier works on FD relaying, e.g., [86] - [89] (for one-way relaying) and [90], [91] (for two-way relaying), assumed the ideal FD case with zero residual SI, which can lead to overestimation of the gains due to FD relaying over HD relaying. The works [51]- [54], [92]- [97] assumed a more practical imperfect SI cancellation with non-zero residual SI. Also, in [51]- [54], the residual SI power is assumed to be proportional with parameter $\beta>0$ to the relay transmit power, which has been validated by the experiments in [49], [50].

Since the residual SI power depends on the relay transmit power, we can see that source and relay power allocation plays a critical role in improving the performance of FD relaying systems. While power allocation for non-buffer FD relaying systems has been extensively studied as reviewed above, power allocation for buffer-aided FD relaying systems has been under-explored. In [89], a buffer-aided FD relaying scheme is proposed, which provides significant throughput gains compared to non-buffer FD relaying schemes. However, zero residual SI and unconstrained relaying delay are assumed. In Chapter 6, we investigate the power allocation problems for buffer-aided FD relaying with imperfect SI cancellation and delay-outage constraint. We investigate two power allocation problems for source arrival rate maximization: (i) Buffer-aided FD relaying with adaptive power allocation when the instantaneous CSI is available at the transmitters (CSIT); (ii) Buffer-aided FD relaying with static power allocation when only statistical CSIT is available. 


\subsection{Chapter Summary}

In this chapter, we have first presented key existing results in resource allocation under delay constraints for source-destination communications link over fading channel. For delay QoS guarantees, average delay or delay-outage constraint can be employed, each of which is suitable for particular set of wireless applications. In the latter case, effective capacity framework is used for developing resource allocation schemes. We next review resource allocation designs for buffer-aided relaying systems. To exploit the buffer-aided relaying capability, adaptive link selection relaying is proposed to efficiently schedule the source-relay link or relay-destination link depending on the instantaneous channel conditions. Such adaptive relaying incurs relaying delay at the relay buffer, which is assumed to be unconstrained or under average delay constraint in existing studies.

In the following Chapters 3-6, we will study novel resource allocation schemes under delay constraints for practical source-destination and 3-node source-relay-destination bufferaided relaying communications systems. In the source-destination communications system, we incorporate data admission control jointly with power allocation to meet both delay and power constraints. The case with energy harvesting transmitter is also considered with average delay or delay-outage constraints. For the relaying systems, we develop resource allocation schemes for half-duplex and full-duplex relaying to support (end-to-end) delayoutage constraint. 


\section{Chapter 3}

\section{Joint Data Admission Control and Power Allocation over Fading Channel under Average Delay Constraint}

In this chapter, we consider a point-to-point communications link over a fading channel with randomly arriving data at the source buffer for transmission to the destination. For delay quality-of-service (QoS) requirement, average delay constraint is imposed. Also, the source is assumed to have (maximum) average power constraint. To avoid constraint violation, it is assumed that only a portion of the arriving data can be buffered (or admitted). Note that the considered data buffer admission control is different from the common user (or stream) admission control. In the latter case, we admit a particular user (among many users) into the system while in the former, we admit data packets of an already admitted user (or stream) into the transmission buffer. This chapter studies the joint data admission control and power allocation (AC-PA) to maximize the throughput defined as the average admitted rate. In particular, we first analyze the structural properties of the optimal AC-PA policy with respect to (w.r.t.) fading channel, data arrival, and queue length states. We then propose an online AC-PA algorithm when the statistical knowledge of the system random channel fading, and data arrival processes is unknown.

In the AC-PA problem, due to the time-varying nature of the channel fading and data

Parts of Chapter 3 are presented at the 2013 IEEE International Conference on Communications (ICC) in Budapest, Hungary [98], and published in the IEEE Transactions on Wireless Communications [99]. 
arrival processes, admission control needs to be done intelligently to balance the throughout and queue length. We can see that increasing the throughput increases the queue length (and hence, the delay) and vice versa. Admission control is even more challenging when the statistical knowledge of the random processes is unknown, which is typical in real-life communications. To address this issue, the AC-PA problem is formulated as an infinite-horizon constrained Markov decision process (MDP) problem, which captures the dynamics of the random processes. We then define so-called post-decision state-value function, which is used to rewrite the Bellman's optimality dynamic programming equation. Using the proposed post-decision state-value function, the monotonicity and convexity of the optimal AC-PA policy w.r.t. the fading channel, data arrival, and queue length states can be studied. The trade-off between maximizing the throughput and minimizing the average queue length (or delay) is also studied. An online AC-PA algorithm is developed, which updates (or learns) the state-value function based on the realizations of the random processes. The algorithm does not require the statistical knowledge of the random processes, and its optimality and convergence are based on the results in stochastic approximation theory. The proposed algorithm is shown to require less storage complexity and converge faster than the conventional Q-learning algorithms [41]. Illustrative results demonstrate that the proposed algorithm achieves higher throughput than the algorithm in [25] under similar delay and power constraints.

The remainder of the chapter is organized as follows. System model and problem formulation are described in Section 3.1. Sections 3.2 details the solution approach and proposes an online learning algorithm. Numerical results are represented in Section 3.3 while Section 3.4 concludes the work.

\subsection{System Model and Problem Formulation}

\subsubsection{Model Description}

We consider a point-to-point communications link where a source transmits data stored in a buffer over a fading channel of bandwidth $B(\mathrm{~Hz})$ to the destination. Transmissions happen over frames of equal duration $T$ (seconds). The model is depicted in Fig. 3.1, where the dynamics of the buffer (or queue) is controlled using admission control and scheduling (or equivalently, power allocation) actions. Specifically, in each frame, the scheduling action 


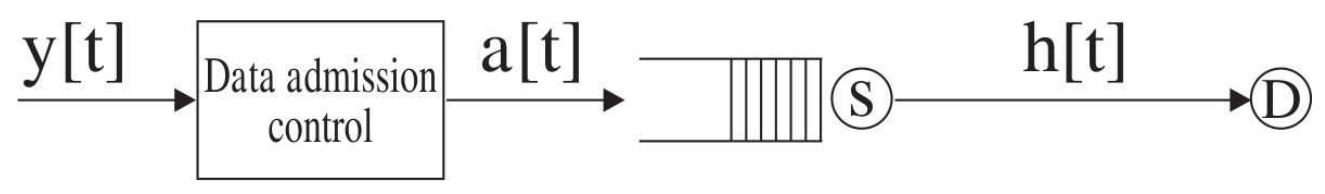

Fig. 3.1: A source-destination communications link with source buffer.

determines the amount of data (or equivalently, the amount of transmit power) removed from the buffer for transmission to the destination. Also, the admission control action determines the amount of data from the newly arriving data to be stored (or admitted) into the buffer. Under the average power constraint, it is clear that there are two conflicting objectives. One objective is to maximize the throughput. The second objective is to minimize the average queue length (or delay). For notional simplicity, we normalize the frame duration $T$ and bandwidth $B$ in the following. Hereafter, we describe the model in detail.

The wireless channel is assumed to be block-fading over the transmission frames. Denote $h[t]$ as the channel state representing the (normalized) channel power gain in frame $t, t=1,2, \ldots$. We assume the channel fading process $\{h[t]\} \in \mathcal{H}$ is stationary ergodic, and independent and identically distributed (i.i.d.) over frames with general probability distribution function (pdf) $p_{\mathcal{H}}(h)$ over the channel state space $\mathcal{H}$, which can be discrete or continuous.

Let $\mathcal{Q} \in[0, \infty)$ denote the queue state space, and let $q[t] \in \mathcal{Q}$ denote the queue state representing the queue length (in number of bits) in frame $t$. We allow the buffer to be an arbitrary real value for mathematical convenience [23], [27]. Let $y[t]$, and $a[t], a[t] \in[0, y[t]]$ (in number of bits) represent the amount of data arrival, and the amount of data admitted into the buffer in frame $t$. We assume the arrival process $\{y[t]\} \in \mathcal{Y}$ is stationary ergodic, and i.i.d. over frames with general pdf $p_{\mathcal{Y}}(y)$ over the data arrival state space $\mathcal{Y}$.

Let $r[t] \in[0, q[t]]$ represent the scheduling action in frame $t$, i.e., the amount of data removed from the buffer and transmitted to the destination. Given $q[1]$ as the initial backlog, the queue dynamics across time slots satisfy the Lindley's recursion:

$$
q[t+1]=q[t]-r[t]+a[t], \forall t
$$

Note that without admission control, $a[t]=y[t], \forall t$. Also, the arriving data in frame $t$ can 
only be scheduled in the next frame earliest.

Given the scheduling action $r[t]$, the corresponding power consumption $P[t]$ in frame $t$ under channel state $h[t]$ is given by the Shannon formula:

$$
P[t]=P(h[t], r[t])=\left(2^{r[t]}-1\right) / h[t], \forall t .
$$

We can see that the power function $P(h, r)$ is convex increasing in $r$ for fixed $h$.

We now define the throughput, average queue length, and average power as follows:

$$
\begin{aligned}
& \bar{R} \triangleq \liminf _{t \rightarrow \infty} \frac{1}{t} \mathbb{E}\left\{\sum_{\tau=1}^{t} a[\tau]\right\} \\
& \bar{Q} \triangleq \limsup _{t \rightarrow \infty} \frac{1}{t} \mathbb{E}\left\{\sum_{\tau=1}^{t} q[\tau]\right\}, \\
& \bar{P} \triangleq \limsup _{t \rightarrow \infty} \frac{1}{t} \mathbb{E}\left\{\sum_{\tau=1}^{t} P[t]\right\} .
\end{aligned}
$$

\subsubsection{Problem Formulation}

The AC-PA problem for throughput maximization under average queue length and power constraints can be posed as:

$$
\max _{r[t] \leq q[t], a[t] \leq y[t], \forall t} \quad \bar{R} \quad \text { s.t. }: \quad \bar{Q} \leq \bar{Q}^{\max }, \quad \bar{P} \leq \bar{P}^{\max }
$$

where $\bar{Q}^{\text {max }}$, and $\bar{P}^{\text {max }}$ are the average queue length and power constraints.

\subsubsection{Optimal Throughput- Delay Trade-off}

We have mentioned the trade-off between maximizing throughput and minimizing the queue length. We now study this trade-off in more details.

Fix some $\bar{P}^{\max }$ and vary $\bar{Q}^{\max }=\bar{Q}$ in $(3.2)$. Let $\bar{R}(\bar{Q})$ denote the corresponding optimal value of $(3.2)$, i.e., $\bar{R}(\bar{Q})$ is the maximum throughput such that the average queue length is less than or equal to $\bar{Q}$. The following proposition characterizes the optimal trade-off $\bar{R}(\bar{Q})$ under average power constraint.

Proposition 3.1. Throughput $\bar{R}(\bar{Q})$ is concave increasing in $\bar{Q}$. 


\section{Joint Data Admission Control and Power Allocation over Fading Channel under Average Delay Constraint}

Proof. Fix $\bar{P}^{\max }$. We can see that the optimal policies will always achieve $\bar{P}^{\text {max }}$. Otherwise, we can increase the scheduling rate to increase the throughput for a similar queue length. We prove $\bar{R}(\bar{Q})$ is concave increasing with $\bar{Q}$. That $\bar{R}(\bar{Q})$ is increasing with $\bar{Q}$ is obvious since more data can be admitted if the queue length is allowed to be larger (for the same scheduling rate). We show that it is concave. Let $\bar{Q}^{1}$ and $\bar{Q}^{2}$ be two queue length values with corresponding throughputs $\bar{R}\left(\bar{Q}^{1}\right)$ and $\bar{R}\left(\bar{Q}^{2}\right)$. We remind that $\bar{R}(\bar{Q})$ is the maximum throughput such that the queue length is less than or equal to $\bar{Q}$. We want to show that for any $\eta \in[0,1]$ :

$$
\bar{R}\left(\eta \bar{Q}^{1}+(1-\eta) \bar{Q}^{2}\right) \geq \eta \bar{R}\left(\bar{Q}^{1}\right)+(1-\eta) \bar{R}\left(\bar{Q}^{2}\right) .
$$

We will prove this using sample path arguments. Let $\{h[t](w)\}_{t=1}^{\infty}$ and $\{y[t](w)\}_{t=1}^{\infty}$ be given sample paths of the channel states and data arrival states. Note that $w$ denotes a sample path of the random process realization. Let $\left\{r_{1}[t](w)\right\}_{t=1}^{\infty}$ and $\left\{a_{1}[t](w)\right\}_{t=1}^{\infty}$ be sequences of control actions corresponding to the policy which attains $\bar{R}\left(\bar{Q}^{1}\right)$. Let $\left\{q_{1}[t](w)\right\}_{t=1}^{\infty}$ be the corresponding sequence of backlog states. Likewise, define $\left\{r_{2}[t](w)\right\}_{t=1}^{\infty},\left\{a_{2}[t](w)\right\}_{t=1}^{\infty}$, and $\left\{q_{2}[t](w)\right\}_{t=1}^{\infty}$ corresponding to $\bar{R}\left(\bar{Q}^{2}\right)$. Note that $r_{i}[t](w) \leq q_{i}[t](w)$ and $a_{i}[t](w) \leq y[t](w)$ for $i=1,2$ for all sample paths $w$ and for all $t$. We have:

$$
\lim _{t \rightarrow \infty} \frac{1}{t} \sum_{\tau=1}^{t} \mathbb{E}\left\{P\left(h[\tau](w), r_{i}[\tau](w)\right)\right\}=\bar{P}^{\max }, \quad i=1,2
$$

where the expectation is taken over all sample paths. Now consider the $\eta$-policy, a new sequences of control actions, $\left\{r_{\eta}[t](w)\right\}_{t=1}^{\infty}$ and $\left\{a_{\eta}[t](w)\right\}_{t=1}^{\infty}$ where for all $t$

$$
\begin{aligned}
r_{\eta}[t](w) & =\eta r_{1}[t](w)+(1-\eta) r_{2}[t](w) \\
a_{\eta}[t](w) & =\eta a_{1}[t](w)+(1-\eta) a_{2}[t](w) .
\end{aligned}
$$

We show that $\eta$-policy is a feasible policy. Let $\left\{q_{\eta}[t](w)\right\}_{t=1}^{\infty}$ be the sequence of backlog states using this policy.

- It can be seen that $a_{\eta}[t](w) \leq y[t](w)$ for all $w$ and $t$.

- For scheduling sequence $\left\{r_{\eta}[t](w)\right\}_{t=1}^{\infty}$, due to the convexity of $P(h, r)$ with $r$ for given $h$, for each $t$, we have:

$$
P\left(h[t](w), r_{\eta}[t](w)\right) \leq \eta P\left(h[t](w), r_{1}[t](w)\right)+(1-\eta) P\left(h[t](w), r_{2}[t](w)\right)
$$


and hence,

$$
\lim _{t \rightarrow \infty} \frac{1}{t} \sum_{\tau=1}^{t} \mathbb{E}\left\{P\left(h[\tau](w), r_{\eta}[\tau](w)\right)\right\} \leq \bar{P}^{\max } .
$$

Hence, the $\eta$-policy satisfies the power constraint.

- Assume at time $t=1, q_{\eta}[1](w)=q_{1}[1](w)=q_{2}[1](w)=0$ for all sample paths $w$. By definition, we have $q_{i}[t+1](w)=q_{i}[t](w)-r_{i}[t](w)+a_{i}[t](w)$ for $i=1,2$ and $t \geq 1$. Then, using recursion, we have $q_{\eta}[t](w)=\eta q_{1}[t](w)+(1-\eta) q_{2}[t](w)$ for all $t$. Consequently, we conclude that $r_{\eta}[t](w)=\eta r_{1}[t](w)+(1-\eta) r_{2}[t](w) \leq q_{\eta}[t](w)$ for all $t$.

Hence, we conclude that $\eta$-policy is a feasible policy.

We have the average queue length by the $\eta$-policy:

$$
\bar{Q}^{\eta}=\lim _{t \rightarrow \infty} \frac{1}{t} \sum_{\tau=1}^{t} \mathbb{E}\left\{q_{\eta}[\tau](w)\right\}=\eta \bar{Q}^{1}+(1-\eta) \bar{Q}^{2}
$$

Summing both sides of (3.5) and taking expectations, we have:

$$
\bar{R}^{\eta}=\lim _{t \rightarrow \infty} \frac{1}{t} \sum_{\tau=1}^{t} \mathbb{E}\left\{r_{\eta}[\tau](w)\right\}=\eta \bar{R}\left(\bar{Q}^{1}\right)+(1-\eta) \bar{R}\left(\bar{Q}^{2}\right)
$$

The $\eta$-policy achieves average queue length $\bar{Q}^{\eta}=\eta \bar{Q}^{1}+(1-\eta) \bar{Q}^{2}$ and throughput $\bar{R}^{\eta}=$ $\eta \bar{R}\left(\bar{Q}^{1}\right)+(1-\eta) \bar{R}\left(\bar{Q}^{2}\right)$. Moreover, by $(3.7)$, the optimal policy with average power $\bar{P}^{\max }$ can achieve the same average queue length but with higher throughput. Thus, we must have $\bar{R}\left(\eta \bar{Q}^{1}+(1-\eta) \bar{Q}^{2}\right) \geq \eta \bar{R}\left(\bar{Q}^{1}\right)+(1-\eta) \bar{R}\left(\bar{Q}^{2}\right)$ as desired. We conclude that $\bar{R}(\bar{Q})$ is concave increasing with $\bar{Q}$.

In the following section, we study the optimal solution of (3.2). 


\subsection{Joint Data Admission Control- Power Allocation}

\subsubsection{MDP-based Optimal Solution}

It can be seen that the problem (3.2) is a constrained MDP. Using [36, Theorem 12.7], the problem (3.2) admits an optimal solution that can be found using the Lagrangian approach:

$$
\min _{\kappa \geq 0, \lambda \geq 0}\left\{\max _{r[t] \leq q[t], a[t] \leq y[t], \forall t}\{\bar{R}-\kappa \bar{Q}-\lambda \bar{P}\}+\kappa \bar{Q}^{\max }+\lambda \bar{P}^{\max }\right\} \text {. }
$$

Therefore, to study (3.10) (and thus (3.2)), we can first study the inner maximization for a given positive multipliers $\kappa$, and $\lambda$ :

$$
\max _{r[t] \leq q[t], a[t] \leq y[t], \forall t}\{\bar{R}-\kappa \bar{Q}-\lambda \bar{P}\} .
$$

In the following sections, we study the optimal solution of (3.11).

In frame $t$, the controller observes the system state $(q[t], h[t], y[t])$ and determines the actions $r[t]$ (or equivalently $P[t]$ ) and $a[t]$ simultaneously [58]. Hence, a stationary control policy can be represented by a 2-tuple function $(r, a): \mathcal{Q} \times \mathcal{H} \times \mathcal{Y} \rightarrow \mathbb{R}^{+} \times \mathbb{R}^{+}$specifying the control actions in slot $t$ as $r[t]=r(q[t], h[t], y[t]) \in[0, q[t]]$ and $a[t]=a(q[t], h[t], y[t]) \in$ $[0, y[t]]$ where $\mathbb{R}^{+}$denotes the set of non-negative numbers.

Define $J(q, h, y)$ as the (pre-decision) state-value function of $(3.11)$, i.e., $J(q, h, y)$ is the optimal value of (3.11) with the starting state $(q[1], h[1], y[1])=(q, h, y)$. The Bellman's optimality dynamic programming equation for (3.11) is:

$J(q, h, y)=\max _{r \leq q, a \leq y}\left\{a-\kappa q-\lambda P(h, r)+\sum_{h^{\prime} \in \mathcal{H}} \sum_{y^{\prime} \in \mathcal{Y}} p_{\mathcal{H}}\left(h^{\prime}\right) p_{\mathcal{Y}}\left(y^{\prime}\right) J\left(q-r+a, h^{\prime}, y^{\prime}\right)\right\}-J\left(q_{0}, h_{0}, y_{0}\right)$

for some arbitrary but fixed state $\left(q_{0}, h_{0}, y_{0}\right)$. The optimal policy is the optimal solution of (3.12). We can see that (3.12) requires known pdfs to evaluate the expectation. However, the pdfs are often unknown in real-time systems which makes the exact computation of the expectation impossible. Conventional reinforcement Q-learning algorithms [41], [59] can be used to learn the optimal AC-PA policy via learning the so-called state-action $Q$ function without requiring known pdfs. Note that after knowing $Q$ function, the state-value 
function $J(q, h, y)$ can also be computed. However, Q-learning algorithms require large complexity, and exhibit slow convergence [27]. We will propose an alternative approach with less complexity and faster convergence in the following.

\subsubsection{Post-decision State-value Function Approach}

Similar to [26], [27], we define the post-decision state-value function $J_{\text {post }}(\check{q})$ as:

$$
J_{\text {post }}(\check{q})=\sum_{h^{\prime} \in \mathcal{H}} \sum_{y^{\prime} \in \mathcal{Y}} p_{\mathcal{H}}\left(h^{\prime}\right) p_{\mathcal{Y}}\left(y^{\prime}\right) J\left(\check{q}, h^{\prime}, y^{\prime}\right)
$$

for post-decision states $\check{q} \in \mathcal{Q}$. The post-decision state $\check{q}[t]$ in frame $t$ is the resulting queue length after the control decisions are made. Hence, we have the queue dynamics as $q[t+1]=\check{q}[t] \triangleq q[t]-r[t]+a[t]$. Using (3.12) and (3.13), the optimal policy can be computed using the state-value function $J_{\text {post }}(\check{q})$ as follows:

$$
\underset{r \leq q, a \leq y}{\arg \max }\left\{a-\kappa q-\lambda P(h, r)+J_{\text {post }}(q-r+a)\right\} \text {. }
$$

As we will see in the following, studying the structural properties of the optimal policy using (3.14) and $J_{\text {post }}(\check{q})$ is easier than using (3.12) and $J(q, h, y)$. Moreover, to compute the optimal policy, it is sufficient to know $J_{\text {post }}(\check{q})$. In the following, we propose an online learning algorithm for $J_{\text {post }}(\check{q})$ without requiring known pdfs. Moreover, as we will see, learning $J_{\text {post }}(\check{q})$ requires less complexity and converges faster than learning the Q function as in the conventional Q-learning algorithms.

From (3.12) and (3.13), we can write the optimality functional equation on $J_{\text {post }}(\check{q})$ :

$$
J_{\text {post }}(\check{q})=\sum_{h^{\prime} \in \mathcal{H}} \sum_{y^{\prime} \in \mathcal{Y}} p_{\mathcal{H}}\left(h^{\prime}\right) p_{\mathcal{Y}}\left(y^{\prime}\right) \max _{r \leq \check{q}, a \leq y^{\prime}}\left\{a-\kappa \check{q}-\lambda P\left(h^{\prime}, r\right)+J_{\text {post }}(\check{q}-r+a)\right\}-J_{\text {post }}\left(\check{q}_{0}\right)
$$

for some arbitrary but fixed state $\check{q}_{0}$.

\subsubsection{Structural Results}

The structural properties of the optimal policy are now stated.

Theorem 3.1. The optimal control policy has the following properties: 


\section{Joint Data Admission Control and Power Allocation over Fading Channel under Average Delay Constraint}

1. $J_{\text {post }}(\check{q})$ is concave decreasing with $\check{q}$.

2. $r^{*}(q, h, y)$ is non-decreasing with $q$ and $y$.

3. $a^{*}(q, h, y)$ is non-increasing with $q$ and non-decreasing with $y$.

4. $r^{*}(q, h, y)$ and $a^{*}(q, h, y)$ are non-decreasing with $h$.

Proof. We prove the decreasing concavity property of $J_{\text {post }}(\check{q})$ with $\check{q} \in \mathcal{Q}$. Note that the monotonic property is obvious. To prove the concavity property, we show that $J_{\text {post }}(\check{q})[t]$ in the RVIA equation (3.16) is concave for $t=1,2, \ldots$ and since $\lim _{t \rightarrow \infty} J_{\text {post }}(\check{q})[t]=J_{\text {post }}(\check{q})$, we conclude that $J_{\text {post }}(\check{q})$ is also concave. We use induction.

By initialization $J_{\text {post }}(\check{q})[1]=0$. Using induction and supposing that $J_{\text {post }}(\check{q})[1]$ is concave for some $t \geq 1$. Hence, for some fixed $h \in \mathcal{H}$, we can easily see that

$$
a-\kappa \check{q}-\lambda P(h, r)+J_{\text {post }}(\check{q}-r+a)[t]
$$

is jointly concave in $(\check{q}, r, a)$ for $r \in[0, \check{q}]$ and $a \in[0, y]$. Hence, the function

$$
\max _{r \leq \breve{q}, a \leq y}\left\{a-\kappa \check{q}-\lambda P(h, r)+J_{\text {post }}(\check{q}-r+a)[t]\right\}
$$

is concave with $\check{q}$ because the maximum of jointly concave function is also concave. Then, from (3.16), we have $J_{\text {post }}(\check{q})[t+1]$ is concave since the expectation preserves the concavity. We conclude that $J_{\text {post }}(\check{q})$ is concave decreasing with $\check{q}$.

We now prove the monotonicity of the control actions. By the concavity of $J_{\text {post }}(\check{q})$, we have the function $a-\kappa q-\lambda P(h, r)+J_{\text {post }}(q-r+a)$ is supermodular in $(q, r)$ for $r \in[0, q]$ and submodular in $(q, a)$ for $a \in[0, y]$. Then, by applying Topkis's Monotonicity Theorem [100, Theorems 1, 2] to (3.14), the scheduling action $r^{*}(q, h, y)$ is non-decreasing with $q$ and the admission control action $a^{*}(q, h, y)$ are non-increasing with $q$. Moreover, that $a^{*}(q, h, y)$ is non-decreasing with $y$ is obvious since when $y$ increases, the optimization domain $[0, y]$ for $a$ becomes larger.

The monotonicity of the control actions with respect to $h$ can be established using the analogous arguments. 
Theorem 3.1 reveals that with the increasing buffer occupancy $q$, more data should be scheduled and less new data should be admitted. When there is more newly arriving data, more data should be scheduled as such to make room for new data to improve the throughput.

\subsubsection{Online Algorithm}

To compute the optimal policy in (3.14), we need to compute the state-value function $J_{\text {post }}(\check{q})$. Using (3.15), $J_{\text {post }}(\check{q})$ can be computed using the sequential relative value iteration algorithm (RVIA) as follows

$$
\begin{aligned}
& J_{\text {post }}(\check{q})[t+1]=\sum_{h^{\prime} \in \mathcal{H}} \sum_{y^{\prime} \in \mathcal{Y}} p_{\mathcal{H}}\left(h^{\prime}\right) p_{\mathcal{Y}}\left(y^{\prime}\right) \max _{r \leq \breve{q}, a \leq y^{\prime}}\left\{a-\kappa \check{q}-\lambda P\left(h^{\prime}, r\right)\right. \\
&\left.+J_{\text {post }}(\check{q}-r+a)[t]\right\}-J_{\text {post }}\left(\check{q}_{0}\right)[t]
\end{aligned}
$$

for $t=1,2, \ldots$ with initial condition $J_{\text {post }}(\check{q})[1]=0$. The purpose of subtracting the scalar offset is to keep the iterations stable. Iterations (3.16) converge to $J_{\text {post }}(\check{q})$ satisfying (3.15) [36].

The iterations (3.16) require known pdfs to evaluate the expectation. However, the equation (3.16) has a nice structure such that the expectations are moved outside of the maximization, and hence, we can use online time-averaging to learn $J_{\text {post }}(\check{q})$ under unknown pdfs, i.e., it solves the MDP (3.11) for fixed $\kappa$, and $\lambda$. Moreover, to find the solution of (3.10), the multipliers $\kappa$, and $\lambda$ can be updated using stochastic sub-gradient method. The optimality and convergence results of the online learning algorithm are ensured using the results in stochastic approximation theory. Using (3.16), the following online control algorithm is employed, which based on the realizations of the random processes.

- Initialization phase: Initialize $J_{\text {post }}(\check{q})[1]$ and $\kappa[1], \lambda[1] \geq 0$, and fix $\check{q}_{0} \in \mathcal{Q}$.

- Data admission control and scheduling phase: In frame $t=1,2, \ldots$, based on the current state $(q[t], h[t], y[t])$, the control action $(r[t], a[t])$ is determined by solving the following problem:

$$
\underset{r \leq q[t], a \leq y[t]}{\arg \max }\left\{a-\kappa[t] q[t]-\lambda[t] P(h[t], r)+J_{\text {post }}(q[t]-r+a)[t]\right\} .
$$


- State-value function updating phase: We update the post-decision state-value function as:

$$
\begin{aligned}
J_{\text {post }}(\check{q})[t+1]= & (1-\phi[t]) J_{\text {post }}(\check{q})[t]+\phi[t]\left(\max _{r \leq \breve{q}, a \leq y[t]}\{a-\kappa[t] \check{q}-\lambda[t] P(h[t], r)\right. \\
& \left.\left.+J_{\text {post }}(\check{q}-r+a)[t]\right\}-J_{\text {post }}\left(\check{q}_{0}\right)[t]\right) .
\end{aligned}
$$

- Multiplier updates: The multipliers $\kappa[t]$, and $\lambda[t]$ are updated as follows:

$$
\begin{aligned}
\lambda[t+1] & =\left[\lambda[t]+\nu[t]\left(P(h[t], r[t])-\bar{P}^{\max }\right)\right]_{0}^{L} \\
\kappa[t+1] & =\left[\kappa[t]+\nu[t]\left(q[t]-\bar{Q}^{\max }\right)\right]_{0}^{L}
\end{aligned}
$$

where $[x]_{a}^{b}$ denotes the projection of $x$ on the interval $[a, b]$ for $a \leq b$, i.e., $[x]_{a}^{b}=a, x$, and $b$ for $x<a, x \in[a, b]$, and $x>b$, respectively, and $L$ is sufficiently large number to ensure boundedness of the multipliers.

The learning rate sequences $\phi[t]$ and $\nu[t]$ satisfy the following properties $[26]$ :

$$
\sum_{\tau=1}^{\infty} \phi[\tau]=\sum_{\tau=1}^{\infty} \nu[\tau]=\infty ; \sum_{\tau=1}^{\infty}(\phi[\tau])^{2}+(\nu[\tau])^{2}<\infty ; \lim _{\tau \rightarrow \infty} \frac{\nu[\tau]}{\phi[\tau]}=0
$$

While the conditions (3.20) guarantee convergence of the proposed algorithm under stationary channel fading statistics, the use of decreasing learning rate sequences may not be viable in practice due to non-stationary channel fading statistics. In such scenarios, alternatively, we can use a fixed and sufficiently small step size. However, the multipliers will only converge to within a (small) neighborhood of the optimal values [101].

It is worth noting that in (3.18), we batch-update $J_{\text {post }}(\check{q})[t+1]$ for all post-decision states $\check{q} \in \mathcal{Q}$, not only the previously-visited state $\check{q}[t]$. This is possible because the traffic arrival and the channel processes are independent of the post-decision queue state $\check{q}[t][27]$. The equation (3.18) can be viewed as stochastic estimate of their counterpart (3.16), and is updated based on instantaneous traffic arrival state $y[t]$ and channel state $h[t]$ without requiring known pdfs.

From the results in stochastic approximation theory and two-timescale analysis [26], [42], 
we can see that in the proposed online allocation algorithm, the state-value function and the multiplier updates converge to the optimal state-value function, and optimal multipliers of (3.10).

The proposed online learning algorithm does not assume any specific pdfs of the system dynamics. Hence, it is very robust to channel and traffic arrival model variations. Due to batch updates, the learning process converges faster. It is mentioned in [27] that batch updates result in twice faster convergence rate than updating one state in each slot. Also, the batch updates preserve the concavity of the value functions, i.e., the functions $J_{\text {post }}(\check{q})[t]$ is concave decreasing for all $t$. Hence, the computational complexity of updating the value functions in (3.18) involves solving convex optimization problems. The convexity preservation of the value functions can also be exploited to derive approximate learning algorithm as in [27]. Compared to Q-learning which learns the state-action $Q$ function with large complexity (which is approximately $|\mathcal{Q}|^{2} \times|\mathcal{H}| \times|\mathcal{Y}|^{2}$ where $|$.$| denotes cardinality of a set) and$ slow convergence [41], [59], the proposed learning requires less complexity (which is $|\mathcal{Q}|$ ) and converges faster. This is because Q-learning maintains a value table for each state-action pair and updates one table entry in each slot.

We can see that the primal variables and the dual Lagrange multipliers are iterated simultaneously albeit on different timescales. The latter is updated at a slower timescale than the former. As seen from the slower timescale variable, the faster timescale variables appear to be equilibrated to the optimal values corresponding to its current value. Also, as viewed from the faster timescale variables, the slower timescale variable appears to be almost constant. Such two timescales updates converge to the optimal solution of (3.10) [26], [42].

\subsection{Illustrative Results}

\subsubsection{Settings}

We implement the proposed learning algorithms using MATLAB. It is assumed Rayleigh fading channel with average channel power gain $10 \mathrm{~dB}$, i.e., $h[t]$ is exponential random variable with $\mathbb{E}\{h[t]\}=10$. We assume (truncated) Poisson arrival process with an average rate of 5 (bits per slot), where the smallest and largest arrival states in each frame are assumed to be 0 and 10 .

The learning rate sequences are chosen as $\phi[t]=(1 / t)^{.7}$ and $\nu[t]=(1 / t)^{.85}$. 


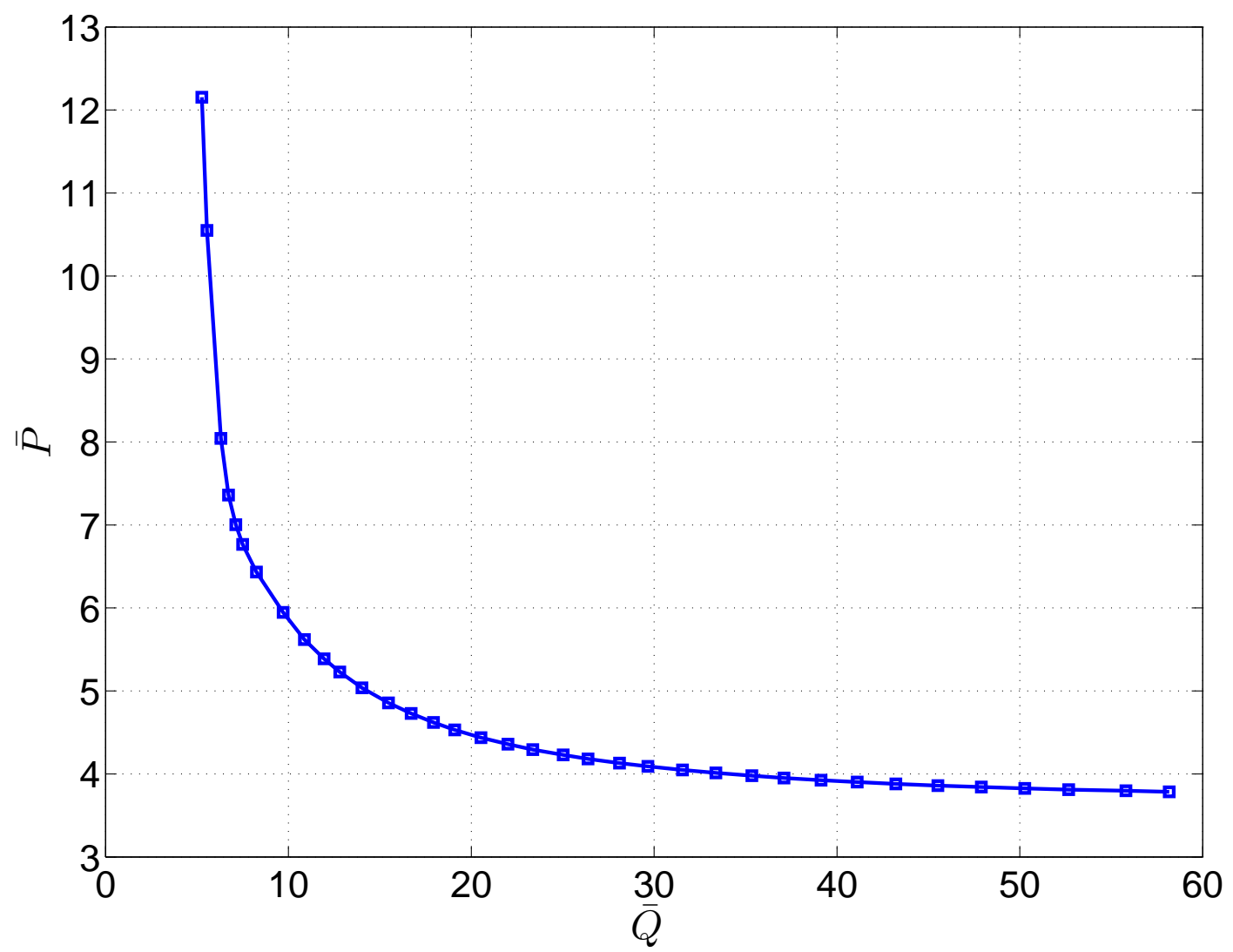

Fig. 3.2: Optimal power- queue length trade-off.

\subsubsection{Numerical Results}

We plot in Fig. 3.2 the optimal power- queue length trade-off [23]. Note that in this case, all data arrivals are buffered and maximum throughput $\bar{R}=5$ (bits) is achieved. We can see that for a given average power $\bar{P}=4.5$ (Watts), the corresponding average queue length $\bar{Q}$ (bits) is approximately 20. It means that if we want to achieve an average queue length smaller than 20 for $\bar{P}^{\max }=4.5$, admission control is required, which reduces the throughput. Also, the minimum average power $\bar{P}(\infty)$ required to ensure finite queue length (or queue stability) without admission control is approximately 3.75 .

We now fix $\bar{P}^{\max }=4.5$. We are looking at the performance of the data admission control and power allocation policy. Fig. 3.3 plots the optimal trade-off curve achieved by the 


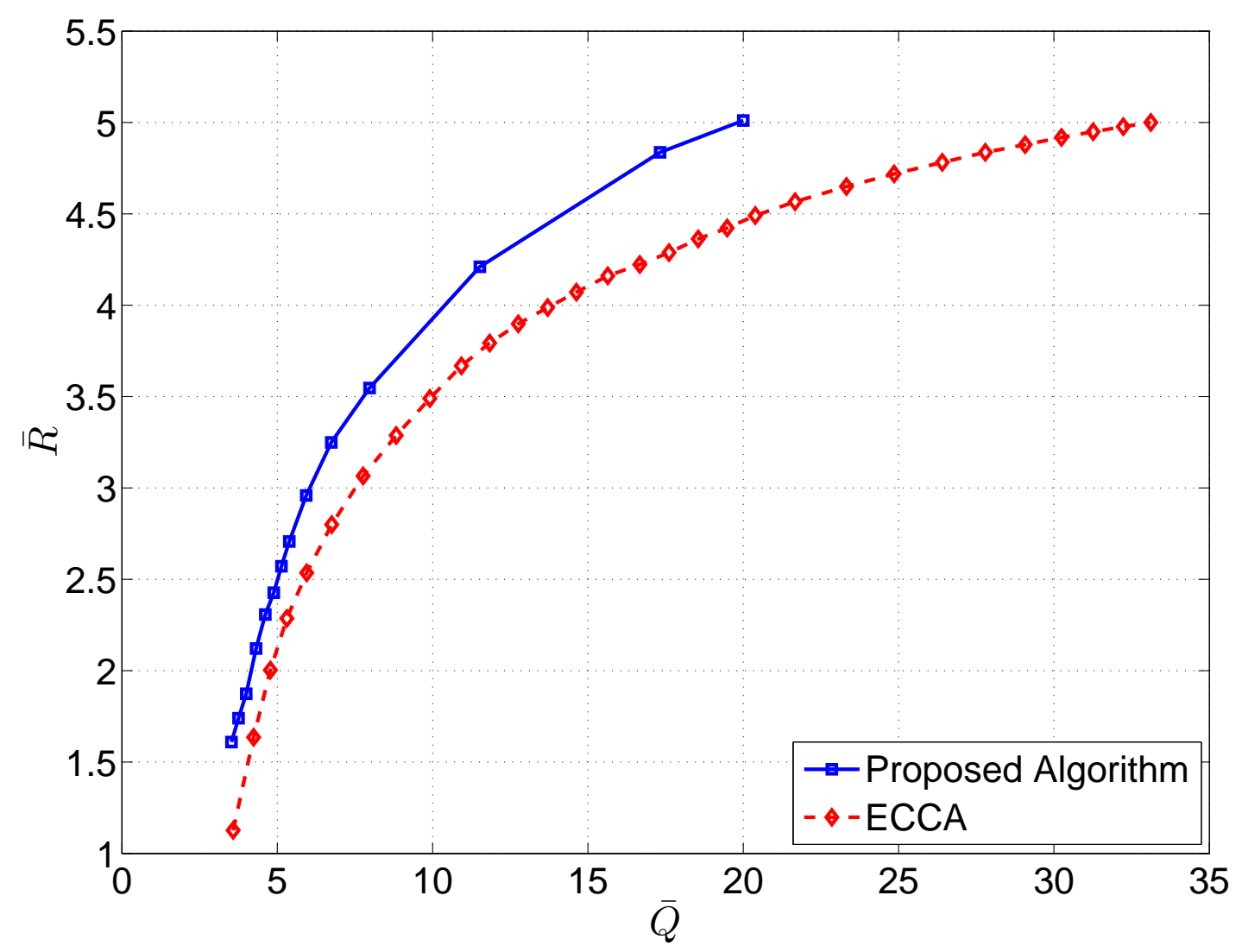

Fig. 3.3: Optimal throughput- queue length trade-off for $\bar{P}^{\max }=4.5$.

proposed online learning algorithm. We also plot the trade-off obtained by the ECCA in [25]. We can observe that for the same (average) queue length, the proposed algorithm is able to achieve higher throughput than the ECCA. Alternatively, for the same throughput, the learning algorithms achieve smaller queue length (and delay). When the average queue length approaches $\bar{Q}=20$, the throughput approaches the average arrival rate or the maximum throughput. In this case, almost all the arrivals are buffered. The results also confirm the concavity increasing characteristic of the optimal trade-off which is analytically proved in Proposition 3.1.

In the last experiment, we demonstrate the use of the proposed learning algorithm to stabilize the queue when the maximum power is $\bar{P}^{\max }=3<\bar{P}(\infty)$. Fig. 3.4 shows the trade-off curves obtained by the proposed algorithm and the ECCA. Again, the proposed 


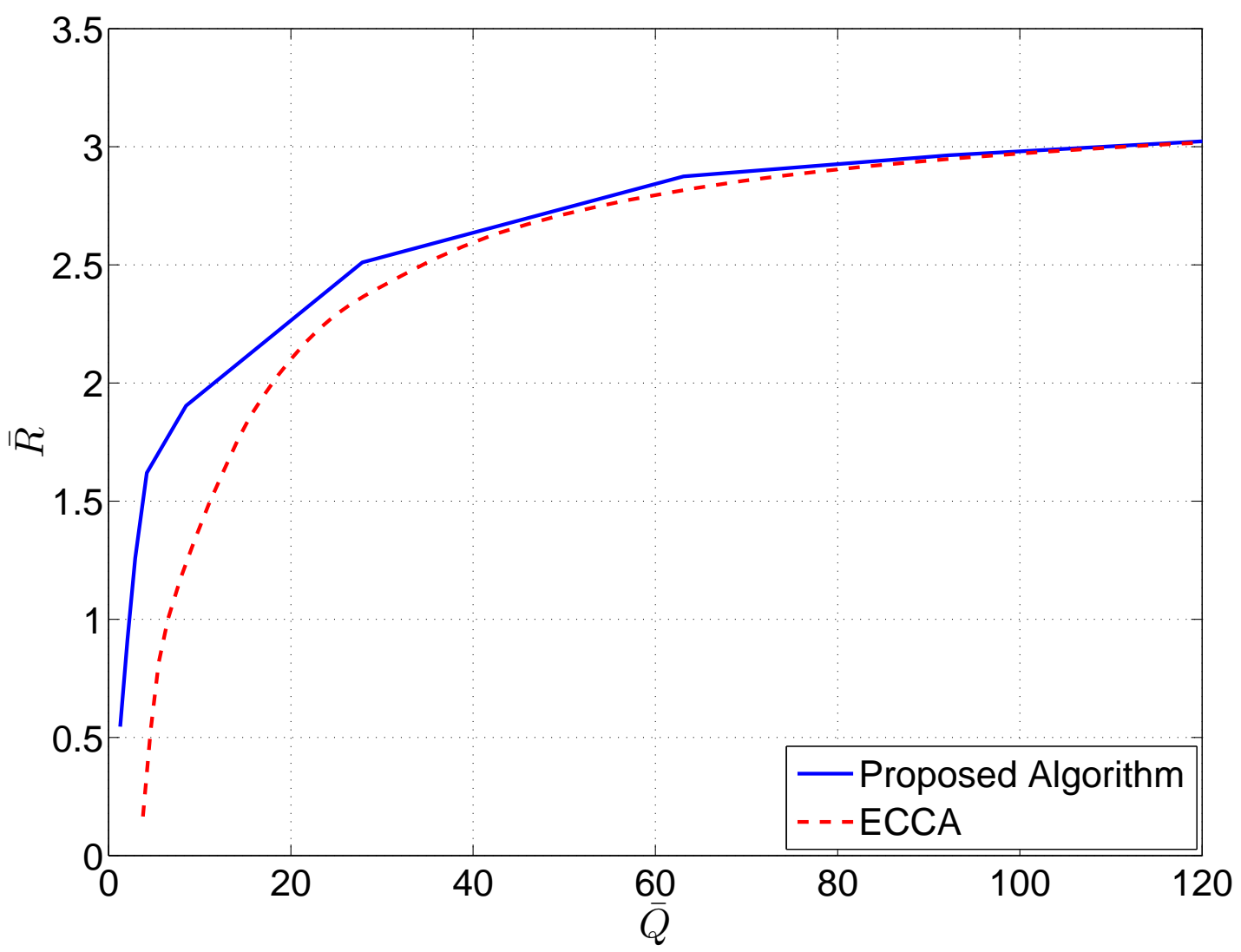

Fig. 3.4: Optimal throughput- queue length trade-off: $\bar{P}^{\max }=3$.

algorithm is more efficient in terms of higher throughput for a given average queue size or smaller average queue size for a given throughput. However, the performance gap is smaller compared to that in Fig. 3.3 for stablizable arrival process.

\subsection{Chapter Summary}

In this chapter, we have presented a study of the joint data admission control- power allocation problem for point-to-point communications link over a fading channel under average delay and power constraints. Using the Markov decision process (MDP) approach and stochastic control tools, we have derived the structural properties of the optimal policy and proposed an online learning algorithm for the optimal policy without requiring known 
statistics of the system random processes. The analysis and algorithm development are relied on the introduction of new state-value function to reformulate the Bellman's dynamic programming equations. Moreover, such state-value function can be learned efficiently using online time-averaging whose convergence, and optimality are ensured by the results in the stochastic approximation theory. The online learning algorithm requires less complexity and converges faster than the conventional Q-learning algorithms. 


\section{Chapter 4}

\section{Power Allocation with Energy Harvesting over Fading Channel under Statistical Delay Constraints}

Next-generation wireless systems are expected to support an ever increasing number of wireless connections with better quality-of-service (QoS), e.g., higher data rate and smaller delay [1], [2]. As a result, energy consumption, as well as energy cost, and greenhouse gas emission are increased, which poses challenges in the design of wireless systems. One promising method to tackle this issue is energy harvesting $(\mathrm{EH})$, where wireless nodes have the capability to harvest energy from the renewable sources (e.g., solar, and thermoelectric, etc) of the surrounding environment, and store the harvested energy in batteries to carry out their functions. In this chapter, we explore power allocation problems for such EH systems to support delay-sensitive communications.

More specifically, this chapter considers an EH system communicating over a fading channel. The stochastic power control problems for source arrival rate maximization under EH and delay constraints are studied. The EH constraint ensures that the randomly available (random in time and amount) renewable energy cannot be spent until it is harvested and subsequently stored in the battery. Also, it cannot spend more energy than the currently available amount in the battery. Moreover, in addition to the average delay constraint model

Parts of Chapter 4 are presented at the 2015 IEEE Global Communications Conference (GLOBECOM) in San Diego, CA, USA [102], and accepted for publication in the IEEE Journal of Selected Areas in Communications, Series on Green Communications and Networking [103]. 


\section{Power Allocation with Energy Harvesting over Fading Channel under Statistical Delay Constraints}

considered in Chapter 3, we also consider delay-outage constraint model. In the latter case, we have converted the original problem into effective capacity maximization problem using asymptotic delay analysis. We formulate the problems as infinite-horizon constrained Markov decision process (MDP) problems. We employ the post-decision state-value function approach in MDP to study the structural properties of the optimal policies, i.e., the monotonicity of the power allocation with channel, EH, and battery states. Throughout this work, it is assumed that the statistics of the system random (channel fading, and EH) processes are unknown to the source. For the model under consideration, reinforcement learning techniques such as $Q$-learning can be employed to optimize the resource dynamically. However, the post-decision state approach is much more appealing than the $Q$-learning as the former approach provides less storage complexity and faster convergence [26], [27]. Towards this end, we develop online power allocation algorithms without requiring known statistics of the random processes. Illustrative results demonstrate the advantages of the proposed approach over existing approaches, i.e., larger arrival rates can be supported under similar channel and EH conditions, and delay constraints.

The remainder of the chapter is organized as follows. Section 4.1 describes the system model and formulates optimization problems for both average delay and delay-outage constraint models. Sections 4.2 and 4.3 describe the solution approaches for the optimization problems, respectively. Numerical results are represented in Section 4.4 while Section 4.5 concludes the work.

\subsection{System Model and Problem Formulations}

\subsubsection{Model Description}

We consider a point-to-point communication system of bandwidth $B(\mathrm{~Hz})$, where the source communicates with the destination, as illustrated in Fig. 4.1. The source is equipped with an EH module, which can harvest renewable energies from the surrounding environment, and then store the harvested energies in an energy queue (or battery). Data is assumed to arrive at the source buffer with the constant rate $\mu$. We consider that the transmission happens over frames of equal duration $T$ (seconds). For notional simplicity, we normalize the frame duration $T$ and bandwidth $B$ in the following. We next describe different parts of the system and their assumptions in detail. 


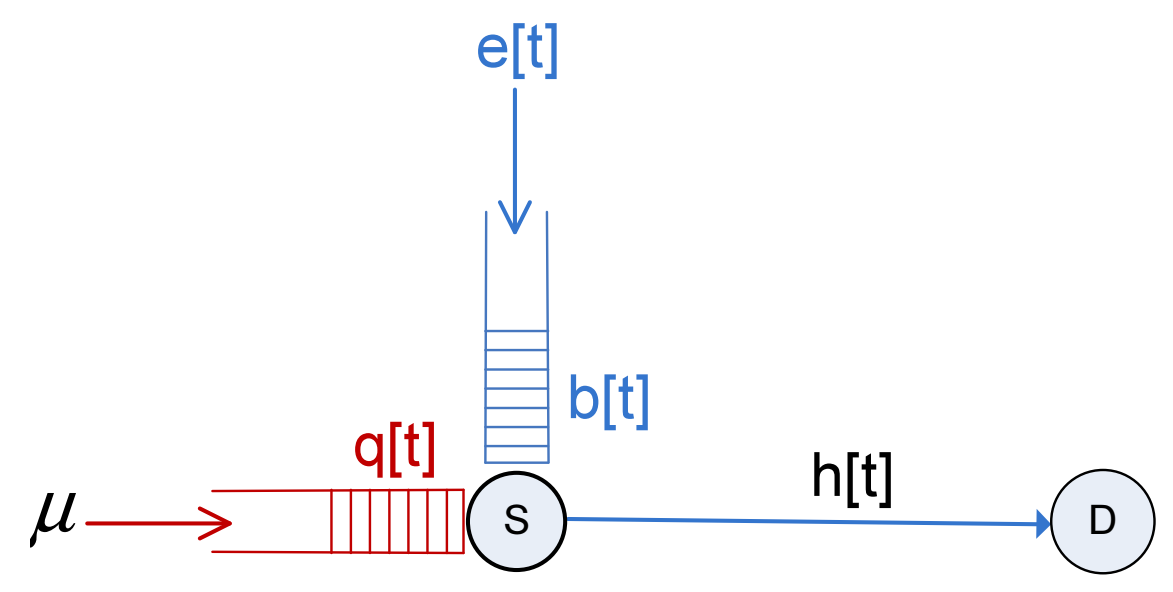

Fig. 4.1: A source-destination communications link with EH transmitter.

1) Channel fading model: We assume block-fading channels with fading duration equal to the frame duration. The channel power gain $h[t]$ in frame $t=1,2, \cdots$ represent the channel state in frame $t$. The channel fading process $\{h[t]\} \in \mathcal{H}$ is assumed to be ergodic, stationary, and i.i.d. with probability distribution function (pdf) $p_{\mathcal{H}}(h)$ over the channel state space $\mathcal{H}$, which can be discrete or continuous.

2) EH and battery model: The source harvests energy amount $e[t]$ from its surroundings during frame $t$. Moreover, $e[t]$ is then stored in a battery and will be available for use in frame $t+1$ onwards. The random EH process $\{e[t]\} \in \mathcal{E}$ is modeled as a stationary, ergodic i.i.d. process with pdf $p_{\mathcal{E}}(e)$ over the $\mathrm{EH}$ state space $\mathcal{E}$. Let $\bar{E}$ denote the average harvested energy in each frame.

Let $b[t] \in \mathcal{B}$ denote the energy amount currently stored in the battery in frame $t$, where $\mathcal{B}$ denotes the battery (energy queue) state space. Let $P[t] \in[0, b[t]]$ denote the transmit power of the source in frame $t$. We assume that the power required for signal processing is negligible compared to the transmit power, and hence, the energies stored and depleted from the battery are only used for data transmissions. The battery dynamics is updated as follows:

$$
b[t+1]=\varphi(b[t], P[t], e[t]), \forall t .
$$

Here $\varphi(\cdot)$ represents a function, which depends on the battery dynamics, e.g., storage efficiency, leakage effects etc. Here, we consider battery with infinite storage capacity. This assumption is with the current trend of the battery technology, where a large amount of en- 


\section{Power Allocation with Energy Harvesting over Fading Channel under Statistical Delay Constraints}

ergy can be stored in the battery with negligible leakage effect, e.g., a super-capacitor [104]. Therefore, as a good approximation in practice, the battery dynamics (4.1) increases and decreases linearly as follows [39], [37], [72]:

$$
b[t+1]=b[t]-P[t]+e[t], \forall t,
$$

We can see that the battery dynamics $\{b[t]\}$ follows a first-order Markov chain that depends only on the present and immediate past conditions. Moreover, when transmitting with power $P[t]$ under channel state $h[t]$, the achievable throughput $r[t]$ is assumed to be given by Shannon's formula:

$$
r[t]=r(h[t], P[t])=\log _{2}(1+P[t] h[t]), \forall t .
$$

Our considered model can be extended for correlated channel fading and correlated EH processes with necessary modifications. In this case, the control actions and state-value functions (considered in Sections 4.2 and 4.3) would include the immediate past channel and/or EH states.

The EH and channel fading processes can vary in different time-scales. In practice, the incoming energy variation is typically slower than that of the channel state. Throughout this work, we consider the scenario of very fast change of the incoming energy, where energy varies in the same time-scale as the channel state. The proposed approaches can be applied with appropriate modifications for the case of slow EH variation.

3) Data queue dynamics: The source utilizes its data buffer to store the traffic arriving with a constant rate $\mu$. Note that the service process of the data queue is $\{r[t]\}$ in (4.3). Let $q[t] \in \mathcal{Q}$ denote the data queue length in frame $t$, where $\mathcal{Q}$ denotes the queue length state space. So, the queue length dynamics can be expressed as follows:

$$
q[t+1]=q[t]-\min \{q[t], r[t]\}+\mu, \forall t
$$

We assume that the queue is stable, i.e., the steady-state queue length random variable $Q$ is bounded. The average queue length $\bar{Q}$ can be expressed as:

$$
\bar{Q}=\lim _{t \rightarrow \infty} \sup \frac{1}{t} \mathbb{E}\left\{\sum_{\tau=1}^{t} q[\tau]\right\} .
$$




\subsubsection{Problem Formulations}

We formulate the stochastic power control problem to maximize the constant arrival rate $\mu$ under the average delay constraint as follows:

$$
\max _{\mu, P[t] \leq b[t], \forall t} \mu \quad \text { s.t.: } \quad \bar{Q} \leq \bar{Q}^{\max }
$$

where $\bar{Q}^{\text {max }}$ is the average queue length bound.

Similarly, the corresponding optimization problem under the delay-outage constraint can be formulated as follows:

$$
\max _{\mu, P[t] \leq b[t], \forall t} \mu \quad \text { s.t.: } \quad \operatorname{Pr}\left(Q>Q^{\max }\right) \leq \zeta_{Q}
$$

where $Q^{\max } \in(0, \infty)$ and $\zeta_{Q} \in(0,1]$ are the queue length bound and queue-length-outage probability, respectively.

We assume that the pdfs of the channel fading and EH processes are unknown to the source. Such assumption makes the solution approach much more challenging as compared to the scenario with known pdfs, for example, in [38], [39]. We solve problems (4.6) and (4.7) optimally in the next two sections and provide intuitive explanations on how to optimally control the transmit power while satisfying the delay and EH constraints without knowing the pdfs of the random processes.

\subsection{Power Allocation under Average Delay Constraint}

\subsubsection{Optimal Allocation Solution}

We observe that problem (4.6) is an infinite-horizon MDP. To this end, it is sufficient that we focus on policies that are independent of time, i.e., stationary policies. The stationary policy $\pi_{\mathrm{A}}$ can be represented by a function $\pi_{\mathrm{A}}: \mathcal{B} \times \mathcal{Q} \times \mathcal{H} \rightarrow \mathbb{R}^{+}$specifying the power control action in frame $t$ as $P[t]=\pi_{\mathrm{A}}(b[t], q[t], h[t])$ such that $P[t] \in[0, b[t]]$, where $\mathbb{R}^{+}$represents the set of non-negative numbers. Furthermore, from (4.4), we can also impose another constraint on $P[t]$ such that $r[t] \leq q[t]$ is satisfied. This finding implies $P[t] \in\left[0, P_{\max }(b[t], q[t], h[t])\right]$, where $P_{\max }(x, y, z)=\min \left\{\left(2^{y}-1\right) / z, x\right\}$. According to [36, Theorem 12.7], the optimal solution of the constrained MDP problem (4.6) can be obtained by exploiting the Lagrangian approach 
as follows:

$$
\min _{\lambda \geq 0}\left\{\max _{\mu, P[t] \leq P_{\max }(b[t], q[t], h[t]), \forall t}\{\mu-\lambda \bar{Q}\}+\lambda \bar{Q}^{\max }\right\}
$$

where $\lambda \geq 0$ represents the Lagrange multiplier associated with constraint average delay constraint. Therefore, to study (4.8), we can first study the inner maximization for a given $\lambda \geq 0$ as follows:

$$
\max _{\mu, P[t] \leq P_{\max }(b[t], q[t], h[t]), \forall t}\{\mu-\lambda \bar{Q}\} .
$$

It is worth mentioning that we update $\lambda$ by sub-gradient method [106]. In the following, we discuss the structural properties of the optimal power allocation policy $\pi_{\mathrm{A}}^{*}$ for (4.9) and show how to allocate the power optimally in each frame $t$.

Let $J(b, q, h)$ denotes the (pre-decision) state-value function for problem (4.9) for a fixed $\lambda>0$. In particular, $J(b, q, h)$ is the optimal value of problem (4.9) with the initial state $(b[1], q[1], h[1])=(b, q, h)$. The Bellman's optimality equation for problem (4.9) can be written as follows [40]:

$$
\begin{array}{r}
J(b, q, h)=\max _{\mu, P \leq P_{\max }(b, q, h)}\left\{\mu-\lambda q+\sum_{\hat{h} \in \mathcal{H}} \sum_{\hat{e} \in \mathcal{E}} p_{\mathcal{H}}(\hat{h}) p_{\mathcal{E}}(\hat{e}) J(b-P+\hat{e}, q-r(h, P)\right. \\
+\mu, \hat{h})\}-J\left(b_{0}, q_{0}, h_{0}\right)
\end{array}
$$

for some fixed state $\left(b_{0}, q_{0}, h_{0}\right)$. The optimal policy $\pi_{\mathrm{A}}^{*}$ is the optimal solution of $(4.10)$.

We now adopt the post-decision state-value function approach in Chapter 3 for the problem under consideration. Similar to (3.13), we define the (post-decision) state-value function $J_{\text {post }}(\check{b}, \check{q})$ from the (pre-decision) state-value function $J(b, q, h)$ as follows:

$$
J_{\text {post }}(\check{b}, \check{q})=\sum_{\hat{h} \in \mathcal{H}} \sum_{\hat{e} \in \mathcal{E}} p_{\mathcal{H}}(\hat{h}) p_{\mathcal{E}}(\hat{e}) J(\check{b}+\hat{e}, \check{q}, \hat{h})
$$

for (post-decision) states $(\check{b}, \breve{q}) \in \mathcal{B} \times \mathcal{Q}$. We have the following relationships on the dynamics of the energy and data queues: $\check{b}[t]=b[t]-P[t]$ and $\check{q}[t]=q[t]-r[t]+\mu$; and $b[t+1]=\check{b}[t]+e[t]$ 
and $q[t+1]=\check{q}[t]$.

Using (4.10) and (4.11), the optimal policy $\pi_{\mathrm{A}}^{*}$ can be computed using $J_{\text {post }}(\check{b}, \check{q})$ as follows:

$$
\underset{\mu, P \leq P \max }{\arg \max (b, h)}\left\{\mu-\lambda q+J_{\text {post }}(b-P, q-r(h, P)+\mu)\right\} .
$$

Before we study the monotonicity of the optimal policy with respect to the data queue length and battery states, we need the following results.

Lemma 4.1. $J_{\text {post }}(\check{b}, \check{q})$ is a concave decreasing function of $\breve{q}$ for a given $\breve{b}$.

Proof. At first, we prove the decreasing monotonic property of $J_{\text {post }}(\check{b}, \check{q})$. The monotonicity is obvious since $\mu-\lambda \check{q}$ is decreasing due to increasing $\breve{q}$. We use induction method to prove the concavity of $J_{\text {post }}(\check{b}, \check{q})$ with respect to $\check{q}$. In particular, we show that $J_{\text {post }}(\check{b}, \check{q})[t]$ in (4.17) is concave in $\check{q}$ for $t=1,2, \ldots$ and since $\lim _{t \rightarrow \infty} J_{\text {post }}(\check{b}, \check{q})[t]=J_{\text {post }}(\check{b}, \check{q})$, we conclude $J_{\text {post }}(\check{b}, \check{q})$ is concave.

We initialize $J_{\text {post }}(\check{b}, \check{q})[t]$ as $J_{\text {post }}(\check{b}, \check{q})[1]=0$. We assume $J_{\text {post }}(\check{b}, \check{q})[t]$ as concave in $\check{q}$ for fixed $\check{b} \in \mathcal{B}$ and $\hat{h} \in \mathcal{H}$. Now, we have to prove $J_{\text {post }}(\check{b}, \check{q})[t+1]$ as concave in $\check{q}$ according to the induction method. Note that $\mu-\lambda \check{q}$ is linear in $\check{q}$ and as we assume that $J_{\text {post }}(\check{b}, \check{q})[t]$ is concave in $\check{q}$, therefore, $\mu-\lambda \check{q}+J_{\text {post }}(\check{b}-P+\hat{e}, \check{q}-r(h, P)+\mu)[t]$ is concave in $\check{q}$ as well. The maximum of a concave function is also a concave function. Hence,

$$
\max _{\mu, P \leq \check{P}_{\max }(\check{b}+\hat{e}, \check{q}, h)}\left\{\mu-\lambda \check{q}+J_{\text {post }}(\check{b}-P+\hat{e}, \check{q}-r(h, P)+\mu)[t]\right\}
$$

is concave in $\check{q}$. Since the expectation operation preserves the concavity property, we conclude $J_{\text {post }}(\check{b}, \check{q})[t+1]$ in $(4.17)$ is concave in $\check{q}$. Therefore, $J_{\text {post }}(\check{b}, \check{q})$ is concave decreasing function in $\check{q}$ for a given $\breve{b}$.

Lemma 4.2. $J_{\text {post }}(\check{b}, \check{q})$ is a concave function of $\check{b}$ for a given $\check{q}$.

Proof. We show the concavity of $J_{\text {post }}(\check{b}, \check{q})$ by the induction method. By following the similar steps to prove Lemma 4.1 , we can show that $J_{\text {post }}(\breve{b}, \check{q})[t]$ in $(4.17)$ is concave in $\check{b}$ for $t=1,2, \ldots$ and since $\lim _{t \rightarrow \infty} J_{\text {post }}(\check{b}, \check{q})[t]=J_{\text {post }}(\check{b}, \check{q})$, we conclude $J_{\text {post }}(\check{b}, \check{q})$ is concave. 


\section{Power Allocation with Energy Harvesting over Fading Channel under Statistical Delay Constraints}

We initialize $J_{\text {post }}(\check{b}, \check{q})=0$ and assume $J_{\text {post }}(\check{b}, \check{q})[t]$ as concave in $\check{b}$ for given values of $\check{q} \in \mathcal{Q}$ and $\hat{h} \in \mathcal{H}$. Next, we have to prove that $J_{\text {post }}(\check{b}, \check{q})[t+1]$ is concave in $\check{b}$ by induction method. As $\mu-\lambda(\check{q}+\mu)$ is independent of $\check{b}$ and $J_{\text {post }}(\check{b}, \check{q})[t]$ is assumed to be concave in $\check{b}$, hence $\mu-\lambda(\check{q}+\mu)+J_{\text {post }}(\check{b}-P+\hat{e}, \check{q}-r(h, P)+\mu)[t]$ is also concave in $\check{b}$. Using similar arguments mentioned in the proof of Lemma 4.1, we can conclude that $J_{\text {post }}(\check{b}-P+\hat{e}, \check{q}-r(h, P)+\mu)[t+1]$ is concave in $\check{b}$ for a given $\check{q}$.

For convenience, let us drop the index of time interval $[t]$ and denote $f(\breve{b}-P)=J_{\text {post }}(\check{b}-$ $P+\hat{e}, \check{q}-r(h, P)+\mu)$. We apply Topkis' monotonicity theorem [100, Theorem 2] to prove that $P$ is a non-decreasing function of $\check{b}$. Therefore, at first, we have to prove that for a given $\hat{h}$ and $\check{q}, J_{\text {post }}(\check{b}, \check{q})$ has an increasing difference in $(\check{b}, P)$ for $P \in[0, \check{b}]$ and a given $\check{q}$. In particular, we need to show

$$
f\left(\check{b}^{\prime}-P^{\prime}\right)-f\left(\check{b}-P^{\prime}\right) \geq f\left(\check{b}^{\prime}-P\right)-f(\check{b}-P), \quad \forall \check{b}^{\prime} \geq \check{b}, \forall P^{\prime} \geq P .
$$

From Lemma 4.1, we know $J_{\text {post }}(\check{b}-P+\hat{e}, \check{q}-r(\hat{h}, P)+\mu)$, i.e., $f(\check{b}-P)$ is concave in $\check{b}$. Hence, from the fundamental property of concave functions, we have [106]:

$$
f(u+\delta)-f(u) \geq f(v+\delta)-f(v), \quad u \leq v, \delta \geq 0 .
$$

Substituting $u=\check{b}-P^{\prime}, v=\check{b}-P$, and $\delta=\check{b}^{\prime}-\check{b}$, we obtain (4.14). Thus, we obtain that $J_{\text {post }}(\check{b}-P+\hat{e}, \check{q}-r(\hat{h}, P)+\mu)$ has an increasing difference in $(\check{b}, P)$ for $P \in[0, \check{b}]$ and a given $\check{q}$.

We now study the monotonicity of the optimal power control policy.

Theorem 4.1. The optimal power control policy $\pi_{A}^{*}$ has the following properties:

1. $\pi_{A}^{*}(b, q, h)$ is a non-decreasing function of $q$ for given $h$ and $b$.

2. $\pi_{A}^{*}(b, q, h)$ is a non-decreasing function of $b$ for given $h$ and $q$.

Proof. Let us consider Lemma 4.1. As $J_{\text {post }}(\check{b}, \check{q})$ is a concave decreasing function of $\check{q}$, hence $\mu-\lambda \check{q}+J_{\text {post }}(\check{b}-P+\hat{e}, \check{q}-r(h, P)+\mu)$ is supermodular in $(\check{q}, r(h, P))$ for $r(h, P) \in[0, \check{q}]$. As $r(h, P)$ is a concave function of $P$, we can say that $\mu-\lambda(\check{q}+\mu)+J_{\text {post }}(\check{b}-P+\hat{e}, \check{q}-r(h, P)+\mu)$ is supermodular in $(\check{q}, P)$ for $P \in\left[0,\left(2^{\check{q}}-1\right) / \hat{h}\right]$ for given $\check{b}$ and $\hat{h}$. As the pre-decision and post-decision parameters are proportional to each other, therefore, we can conclude that $\pi_{\mathrm{A}}^{*}(b, q, h)$ is a non-decreasing function of $q$ for given $h$ and $b$. 
Next, to show that $\pi_{\mathrm{A}}^{*}(b, q, h)$ is a non-decreasing function of $b$ for given $h$ and $q$, consider Lemma 4.2. By applying Topkis' monotonicity theorem [100, Theorem 2] and representing the parameters in terms of pre-decision state, we conclude that $\pi_{\mathrm{A}}^{*}(b, q, h)$ is a non-decreasing function of $b$ for given $h$ and $q$.

Theorem 4.1 prescribes that more power is used for transmission for a given channel and available energy when there are more data-bits in the data-queue. In other words, with the increasing buffer occupancy $q$, more data should be scheduled to provide more 'room' for new incoming data traffic without violating the delay constraint. We also observe from Theorem 4.1 that we should increase transmit power for a given channel and data-queue condition if we have more energy in the battery. These findings help to reduce the search space to solve (4.12) by restricting the search space towards specified direction. Intuitively, Theorem 4.1 helps to reduce the data queue length in order to meet the average delay constraint.

\subsubsection{Online Algorithm}

Now we propose an online algorithm to obtain the optimal policy $\pi_{\mathrm{A}}^{*}$ without requiring known statistics of the underlying random processes. It is equivalent to learn $J_{\text {post }}(\check{b}, \check{q})$ since we can obtain $\pi_{\mathrm{A}}^{*}$ from $J_{\text {post }}(\check{b}, \check{q})$ using $(4.12)$.

From the relationship (4.11), we first write the optimality equation for $J_{\text {post }}(\check{b}, \check{q})$ as follows:

$$
\begin{aligned}
J_{\text {post }}(\check{b}, \check{q})=\sum_{\hat{h} \in \mathcal{H}} \sum_{\hat{e} \in \mathcal{E}} p_{\mathcal{H}}(\hat{h}) p_{\mathcal{E}}(\hat{e}) \max _{\mu, P \leq P_{\max }(\check{b}+\hat{e}, \check{q}, \hat{h})}\left\{\mu-\lambda \check{q}+J_{\text {post }}(\check{b}+\hat{e}-P, \check{q}\right. \\
-r(\hat{h}, P)+\mu)\}-J_{\text {post }}\left(\check{b}_{0}, \check{q}_{0}\right)
\end{aligned}
$$

for some fixed state $\left(\check{b}_{0}, \check{q}_{0}\right)$. Toward this end, notice that when the statistics of the channel fading and $\mathrm{EH}$ processes is known, $J_{\text {post }}(\check{b}, \check{q})$ can be computed using the sequential relative 
value iteration algorithm (RVIA) as follows

$$
\begin{array}{r}
J_{\text {post }}(\check{b}, \check{q})[t+1]=\sum_{\hat{h} \in \mathcal{H}} \sum_{\hat{e} \in \mathcal{E}} p_{\mathcal{H}}(\hat{h}) p_{\mathcal{E}}(\hat{e}) \max _{\mu, P \leq P_{\max }(\check{b}+\hat{e}, \check{q}, \hat{h})}\left\{\mu-\lambda \check{q}+J_{\text {post }}(\check{b}+\hat{e}-P,\right. \\
\check{q}-r(\hat{h}, P)+\mu)[t]\}-J_{\text {post }}\left(\check{b}_{0}, \check{q}_{0}\right)[t],
\end{array}
$$

for $t=1,2, \ldots$ with initial value function $J_{\text {post }}(\check{b}, \check{q})[1]$.

Using the post-decision approach helps reducing the number of states to compute the state-value function, as we do not need to keep track of the channel states over the time intervals in $J_{\text {post }}(\check{b}, \check{q})[t]$ to achieve the optimal state-value function. Learning the value function $J(b, q, h)[t]$ as in the conventional Q-learning approach would ultimately increase the computational complexity to a large extent.

We now resort to an online time-averaging algorithm to obtain (4.17) without requiring known fading and EH distributions. We now describe the implementation strategy of the proposed online algorithm as follows:

- Initialization phase: Initialize $J_{\text {post }}(\check{b}, \check{q})[1]$ and $\lambda[1] \geq 0$, and fix $\left(\check{b}_{0}, \check{q}_{0}\right) \in \mathcal{B} \times \mathcal{Q}$.

- Transmission phase: For $t=1,2, \ldots$, based on the current state $(b[t], q[t], h[t])$, the optimal power control action $P^{*}[t]$ is determined by solving the following problem:

$$
\underset{\mu, P[t] \leq P_{\max }(b[t], q[t], h[t])}{\arg \max }\left\{\mu-\lambda q[t]+J_{\text {post }}(b[t]-P[t], q[t]-r(h[t], P[t])+\mu)[t]\right\} .
$$

- State-value function updating phase: We update the state-value function as:

$$
\begin{gathered}
J_{\text {post }}(\check{b}, \check{q})[t+1]=(1-\phi[t]) J_{\text {post }}(\check{b}, \check{q})[t]+\phi[t]\left(\max _{\mu, P \leq P_{\max }(\check{b}+\hat{e}, \check{q}, \hat{h})}\{\mu-\lambda[t] \check{q}+\right. \\
\left.\left.J_{\text {post }}(\check{b}+\hat{e}[t]-P, \check{q}-r(h[t], P)+\mu)[t]\right\}-J_{\text {post }}\left(\check{b}_{0}, \check{q}_{0}\right)[t]\right) .
\end{gathered}
$$


- Multiplier update: The multiplier $\lambda[t]$ is updated as follows:

$$
\lambda[t+1]=\left[\lambda[t]+\nu[t]\left(q[t]-\bar{Q}^{\max }\right)\right]_{0}^{L}
$$

where $[x]_{a}^{b}$ denotes the projection of $x$ on the interval $[a, b]$ for $a \leq b$ and $L$ is sufficiently large number to ensure boundedness of the multiplier.

The learning rate sequences $\phi[t]$ and $\nu[t]$ represent the decreasing step-size parameters for the value-iteration function and the Lagrange multiplier update equation, respectively. The step-size parameters satisfy the following properties [26]:

$$
\sum_{t=1}^{\infty} \phi[t]=\sum_{t=1}^{\infty} \nu[t]=\infty ; \quad \sum_{t=1}^{\infty}(\phi[t])^{2}+(\nu[t])^{2}<\infty ; \quad \lim _{t \rightarrow \infty} \frac{\nu[t]}{\phi[t]}=0 .
$$

We can see that (4.19), being a stochastic estimate of (4.17), is updated based on the instantaneous realizations of the underlying random processes without requiring their statistics. Moreover, this algorithm is applicable to any distributions of the channel fading and EH processes, and hence, is considered as robust to the variations of channel fading and EH models. The convergence of proposed online algorithm to $J_{\text {post }}(\check{b}, \check{q})$ satisfying $(4.16)$ can be found by following the similar steps described in [26, Appendix].

\subsubsection{Baseline Transmission Schemes}

To show the effectiveness of the developed optimal power control scheme for the average delay model by simulations in Section 4.4, we propose two baseline schemes in this subsection based on the results available in the existing literature. The first baseline scheme, namely benchmark scheme, does not keep track of the battery states in each time interval to achieve the optimal throughput. Therefore, instead of constraining the instantaneous transmit power, the average power consumption is upper bounded by the average harvested energy. We show by simulations that our developed scheme and the benchmark scheme result in the same optimal throughput for a given average delay requirement. The second baseline scheme, denoted as naive scheme, heuristically calculates the transmit power as a function of the amount of remaining energy at the battery and the number of bits to be transmitted.

1) Benchmark scheme: It has been shown in [105] that the optimal utility of an EH system can be calculated by knowing the average harvested energy only without requiring 


\section{Power Allocation with Energy Harvesting over Fading Channel under Statistical Delay Constraints}

the dynamics of the battery to be considered. Based on this finding, we formulate an optimization problem for the benchmark scheme as follows:

$$
\max _{\mu, P[t]} \quad \mu \quad \text { s.t.: } \quad \mathbb{E}\{P[t]\} \leq \bar{E}, \bar{Q} \leq \bar{Q}^{\max }
$$

where we remind that $\bar{E}$ is the average harvested energy in each frame. Note that the difference between problems (4.22) and (4.6) is that problem (4.6) keeps track of both the battery and queue length states in each time interval, whereas problem (4.22) keeps track of the queue length state only. Therefore, the computational complexity to solve problem (4.22) is much less than that involved to solve problem (4.6). The optimal solution of problem (4.22) can be obtained by using the Lagrangian approach as follows:

$$
\min _{\zeta \geq 0, \psi \geq 0}\left\{\max _{\mu, P[t]}\{\mu-\zeta \bar{Q}-\psi \mathbb{E}\{P[t]\}\}+\zeta \bar{Q}^{\max }+\psi \bar{E}\right\},
$$

where $\zeta$ and $\psi$ are the multipliers. The optimal solution for the benchmark scheme can be obtained by exploiting the same post-decision state approach as used in the scheme developed in Section 4.2.1.

Problem (4.23) is a single-dimensional constrained MDP as opposed to the two-dimensional MDP (4.9). Therefore, the post-decision state approach is only a function of the queue length state $q$ for given $\zeta$ and $\psi$. As such, the computational complexity to update the post-decision state is much less than the developed scheme.

In this benchmark scheme, it is implicitly assumed that the available energy in each time interval is infinite, even if the average transmit power is constrained by finite $\bar{E}$. It is worth mentioning that the benchmark scheme is a theoretical abstraction to obtain the optimal throughput for an EH system. Hence, this scheme cannot be applied in real-time systems (as may not be feasible for certain time intervals), where the available energy in each time interval depends on the random EH process and the past-control actions. Nonetheless, the reason to consider the benchmark scheme in this paper is to show that our scheme can achieve the same optimal throughput as that offered by the benchmark scheme. In fact, our developed online algorithm in Section 4.2.2 takes into account the dynamics of the available energy, unlike the benchmark scheme while providing the same optimal solution. We show this comparison in detail in Section 4.4. 
2) Naive Scheme: In this scheme, in each frame $t$, we assign $P[t]=\min \left\{b[t], \frac{2^{q[t]}-1}{h[t]}\right\}$. This scheme neither takes into account the impact of channel and energy arrival statistics nor apply any learning technique to improve the power control policy. The purpose of considering the naive scheme is to show the effectiveness of controlling the transmit power intelligently, as developed in our proposed scheme, over the transmission frames.

\subsection{Power Allocation under Delay-Outage Constraint}

In this section, we propose an approach to solve problem (4.7).

\subsubsection{Effective Capacity Maximization}

In order to handle the delay-outage constraint, we need to study the tail distribution of steady-state queue length random variable $Q$, which is very cumbersome. To overcome this difficulty, we assume large delay region, i.e., $Q^{\max }$ is sufficiently large, and employ the asymptotic delay analysis. More specifically, using (2.6), the problem (4.7) can be reformulated as the effective capacity maximization problem as follows:

$$
\max _{P[t] \leq b[t], \forall t}-\frac{1}{\theta^{\operatorname{tar}}} \log \mathbb{E}\left\{e^{\left.-\theta^{\operatorname{tar} r} r t\right]}\right\}, \quad \theta^{\operatorname{tar}} \triangleq-\log \left(\zeta_{Q}\right) / Q^{\max }
$$

where $r[t]$ is given by (4.3). In the following, for the sake of convenience and generalization, let us denote the normalized delay exponent as $\theta=\theta^{\operatorname{tar}} / \log (2)$. Using the monotonicity of $\log (\cdot)$, problem $(4.24)$ can be re-expressed as follows:

$$
\min _{P[t] \leq b[t], \forall t} \mathbb{E}\left\{(1+h[t] P[t])^{-\theta}\right\}
$$

We can now observe that problem (4.25) is an infinite-horizon MDP. In the following, we present an approach to solve and analyze problem (4.25).

We focus on the stationary policies $\pi_{\mathrm{S}}$ for problem (4.25). The policy $\pi_{\mathrm{S}}$ can be represented by function $\pi_{\mathrm{S}}: \mathcal{B} \times \mathcal{H} \rightarrow \mathbb{R}^{+}$specifying the power control action in frame $t$ as $P[t]=\pi_{\mathrm{S}}(b[t], h[t])$ such that $P[t] \in[0, b[t]]$. Note that in contrast to $\pi_{\mathrm{A}}$ (the policy for the average delay constraint model), $\pi_{\mathrm{S}}$ does not depend on $\mathcal{Q}$ and hence is not a function of $q[t]$. The optimal value of $\mu$ for a given feasible policy $\pi_{\mathrm{S}}$ obtained from problem (4.25) represents the effective capacity of the considered EH system [32], [61]. Note that when 
$\theta \rightarrow 0$, i.e., no constraint is imposed on the delay requirement, the solution of problem (4.25) can also be obtained from the classical optimal online schemes described in [38], [39] for a sufficiently large number of transmission frames and for a known channel fading and EH statistics. Similar to the average delay constraint model, we assume that the channel fading and EH statistics are unknown for the delay-outage constraint model as well.

Let $V(b, h)$ denotes the (pre-decision) state-value function for problem $(4.25)$, i.e., $V(b, h)$ is the optimal value of problem (4.25) with the initial state $(b[1], h[1])=(b, h)$. The Bellman's optimality equation for problem (4.25) can be written as follows [40]:

$$
V(b, h)=\min _{P \leq b}\left\{(1+h P)^{-\theta}+\sum_{\hat{h} \in \mathcal{H}} \sum_{\hat{e} \in \mathcal{E}} p_{\mathcal{H}}(\hat{h}) p_{\mathcal{E}}(\hat{e}) V(b-P+\hat{e}, \hat{h})\right\}-V\left(b_{0}, h_{0}\right)
$$

for a fixed state $\left(b_{0}, h_{0}\right)$. The optimal policy $\pi_{\mathrm{S}}^{*}$ is the optimal solution of (4.26).

\subsubsection{Post-decision State-value Function Approach}

Similar to the average delay model, we adopt the post-decision state-value approach for the delay-outage constraint model to optimally control the transmit power. The post-decision state-value function for delay-outage constraint model $V_{\text {post }}(\check{b})$ can be defined as follows:

$$
V_{\text {post }}(\check{b})=\sum_{\hat{h} \in \mathcal{H}} \sum_{\hat{e} \in \mathcal{E}} p_{\mathcal{H}}(\hat{h}) p_{\mathcal{E}}(\hat{e}) V(\check{b}+\hat{e}, \hat{h})
$$

for post-decision states $\check{b} \in \mathcal{B}$. The dynamics of the battery can be represented as $\check{b}[t]=$ $b[t]-P[t]$, and $b[t+1]=\check{b}[t]+e[t]$. Using (4.26) and (4.27), $\pi_{\mathrm{S}}^{*}$ can be computed as:

$$
\underset{P \leq b}{\arg \min }\left\{(1+h P)^{-\theta}+V_{\text {post }}(b-P)\right\} \text {. }
$$

Lemma 4.3. $V_{\text {post }}(\check{b})$ is a convex decreasing function of $\breve{b}$.

Proof. At first, we prove the decreasing monotonic property of $V_{\text {post }}(\check{b})$. The monotonicity is obvious since $(1+\hat{h} P)^{-\theta}$ is decreasing due to increasing $P$, which is proportional to the stored energy. We use induction method to prove the convexity of $V_{\text {post }}(\breve{b})$. In particular, we 


\section{Power Allocation with Energy Harvesting over Fading Channel under Statistical Delay Constraints}

show that $V_{\text {post }}(\check{b})[t]$ in $(4.31)$ is convex for $t=1,2, \ldots$, and since $\lim _{t \rightarrow \infty} V_{\text {post }}(\check{b})[t]=V_{\text {post }}(\check{b})$, we conclude $V_{\text {post }}(\check{b})$ is convex.

We initialize $V_{\text {post }}(\check{b})[t]$ as $V_{\text {post }}(\check{b})[1]=0$. We assume $V_{\text {post }}(\check{b})[t]$ as convex for a given $\hat{h} \in \mathcal{H}$. Now, we have to prove $V_{\text {post }}(\check{b})[t+1]$ as convex according to the induction method. Note that $(1+\hat{h} P)^{-\theta}$ is convex in $P$, and as we assume $V_{\text {post }}(\check{b})[t]$ is convex in $\breve{b}$, therefore, we conclude $(1+\hat{h} P)^{-\theta}+V_{\text {post }}(\breve{b}-P+\hat{e})[t]$ is jointly convex in $P$ and $\breve{b}$ for $P \in[0, \breve{b}][106]$. Moreover, the minimum of jointly convex function is convex. Hence,

$$
\min _{P \leq \breve{b}+\hat{e}}\left\{(1+\hat{h} P)^{-\theta}+V_{\text {post }}(\check{b}+\hat{e}-P)\right\}
$$

is convex with $\breve{b}$. Then, from $(4.31)$, we conclude $V_{\text {post }}(\check{b})[t]$ is convex, since the expectation operation preserves the convexity property. So, we conclude $V_{\text {post }}(\check{b})$ is a convex decreasing function of $\check{b}$.

Theorem 4.2. The optimal control policy $\pi_{S}^{*}(b, h)$ is a non-decreasing function of $b$ for a given $h$.

Proof. Using [100, Lemma 1] and Lemma 4.3 , we can show that $(1+\hat{h} P)^{-\theta}+V_{\text {post }}(\check{b}-$ $P+\hat{e})$ is an increasing difference function in $(\breve{b}, P)$ for $P \in[0, \check{b}+\hat{e}]$. Then by applying Topkis' monotonicity theorem [100, Theorem 2], we deduce that the control action $P$ is nondecreasing with $\breve{b}$ for a given $\hat{h}$. Intuitively, $P$ is non-decreasing with $\breve{b}$ is obvious, since when $\check{b}$ increases, the optimization domain $[0, \check{b}+\hat{e}]$ for $P$ becomes larger. The larger set helps to reduce $(1+h P)^{-\theta}+V_{\text {post }}(\check{b}-P+\hat{e})$ more. Hence, representing the parameters in terms of pre-decision state, we can conclude that $\pi_{\mathrm{S}}^{*}(b, h)$ is non-decreasing with $b$ for a given $h$.

Similar to Theorem 4.1, we observe that we should allocate more power for transmission to increase the throughput if we have more energy available in the battery. This finding helps to reduce the computational complexity to solve (4.28) as we restrict the search space towards one direction to achieve the optimal solution.

Lemma 4.3 and Theorem 4.2 provide insights about the structural properties of the post-decision state-value function $V_{\text {post }}(\check{b})$ and the optimal power control policy towards developing the online algorithm in Section 4.3.2. However, as the post-decision state-value function $V_{\text {post }}(\check{b})$ is a convex decreasing function of $\breve{b}$, following the approximation method 
developed in [27], we can approximate $V_{\text {post }}(\check{b})$ as a successive convex function to alleviate the computational complexity and develop a suboptimal online algorithm.

\subsubsection{Online Algorithm}

We propose an online algorithm to obtain the optimal policy $\pi_{\mathrm{S}}^{*}$ under the delay-outage constraint.

From (4.26) and (4.27), we can write the optimality equation for delay-outage constraint model as follows:

$$
V_{\text {post }}(\check{b})=\sum_{\hat{h} \in \mathcal{H}} \sum_{\hat{e} \in \mathcal{E}} p_{\mathcal{H}}(\hat{h}) p_{\mathcal{E}}(\hat{e}) \min _{P \leq \check{b}+\hat{e}}\left\{(1+\hat{h} P)^{-\theta}+V_{\text {post }}(\check{b}+\hat{e}-P)\right\}-V_{\text {post }}\left(\check{b}_{0}\right)
$$

for some fixed state $\breve{b}_{0}$.

Notice that when the channel and EH processes are known, $V_{\text {post }}(\check{b})$ can be computed using the sequential RVIA as follows for $t=1,2, \cdots$ :

$$
V_{\text {post }}(\check{b})[t+1]=\sum_{\hat{h} \in \mathcal{H}} \sum_{\hat{e} \in \mathcal{E}} p_{\mathcal{H}}(\hat{h}) p_{\mathcal{E}}(\hat{e}) \min _{P \leq \check{b}+\hat{e}}\left\{(1+\hat{h} P)^{-\theta}+V_{\text {post }}(\check{b}+\hat{e}-P)[t]\right\}-V_{\text {post }}\left(\check{b}_{0}\right)[t],
$$

with initial value function $V_{\text {post }}(\check{b})[1]$. For online implementation, we can follow the same procedures, i.e., initialization, transmission, and learning phases as described for the average delay model in Section 4.2 .

\subsubsection{Baseline Transmission Schemes}

Based on the concepts behind developing the baseline schemes for the average delay model, we describe two similar types of baseline schemes for the delay-outage constraint model to show the effectiveness of our developed scheme.

1) Benchmark Scheme: It has been shown in [105] that the optimal utility of an EH system can be calculated by knowing the average harvested energy only without requiring the exact distribution of the EH process to be known. Hence, with a given average harvested 
energy $\bar{E}$, we formulate an optimization problem for the 'benchmark scheme' as follows:

$$
\min _{P[t] \geq 0} \mathbb{E}\left\{(1+h[t] P[t])^{-\theta}\right\} \quad \text { s.t. : } \quad \mathbb{E}\{P[t]\} \leq \bar{E} .
$$

The Lagrangian of problem (4.32) is given by

$$
\mathcal{L}=\mathbb{E}\left\{(1+h[t] P[t])^{-\theta}\right\}+\eta(\mathbb{E}\{P[t]\}-\bar{E})
$$

where $\eta$ represents Lagrange multiplier associated with the only constraint of problem (4.32). Applying Karush-Kuhn-Tucker (KKT) optimality conditions [106], we obtain optimal $P[t]$ as follows:

$$
P^{*}[t]=\left\{\begin{array}{lr}
\left(\frac{\theta}{\eta(h[t])^{\theta}}\right)^{\frac{1}{1+\theta}}-\frac{1}{h[t]}, & \text { if } h[t] \geq \frac{\eta}{\theta} \\
0, & \text { otherwise. }
\end{array}\right.
$$

From the KKT optimality conditions, we can show that the constraint of problem (4.32) is satisfied with equality at the optimal point. Hence, the optimal solution of $\eta$ can be obtained numerically by solving the following equation

$$
\int_{\frac{\eta}{\theta}}^{\infty}\left(\left(\frac{\theta}{\eta h^{\theta}}\right)^{\frac{1}{1+\theta}}-\frac{1}{h}\right) p_{\mathcal{H}}(h) d h=\bar{E} .
$$

We can see that combining (4.34) and (4.35) provides the same result as that obtained for a non-EH system, e.g., [61, Eq. (8), (9)], if the average available energy is replaced by the average harvested energy $\bar{E}$. Note that in this benchmark scheme, it is assumed that the available energy in each frame is infinite (see (4.34)), even if the average energy is constrained by $\bar{E}$. Hence, similar to the average delay model, this scheme cannot be applied in real-time systems when the available energy in each frame depends on realizations of the random EH process and the past control actions.

2) Offline Scheme: In this baseline scheme, we formulate an offline optimization problem motivated by the contributions made in [38], [39] for performance comparison. This offline scheme was originally proposed for finite number of transmission time intervals in [38], [39]. However, we consider infinite time horizon. Hence, to make a fair comparison in the numerical results, we consider a large number of time intervals for this scheme to compare 


\section{Power Allocation with Energy Harvesting over Fading Channel under Statistical Delay Constraints}

its performance with the developed and benchmark schemes. We formulate an optimization problem as follows:

$$
\begin{array}{ll}
\min _{P[t] \geq 0, \forall t} & \frac{1}{\mathcal{T}} \sum_{t=1}^{\mathcal{T}}(1+h[t] P[t])^{-\theta} \\
\text { s.t. } & \sum_{k=1}^{t} P[k] \leq \sum_{k=0}^{t-1} e[k], t=1, \ldots, \mathcal{T}
\end{array}
$$

where $\mathcal{T}$ denotes the maximum number of time intervals. Problem (4.36) is a convex optimization problem and hence can be solved optimally and efficiently [106]. Applying KKT optimality condition in problem $(4.36)$, we can obtain optimal power allocation $P^{*}[t]$ as follows:

$$
P^{*}[t]= \begin{cases}\left(\frac{\theta}{(h[t])^{\theta} \sum_{k=t}^{\mathcal{T}} \lambda[k]}\right)^{\frac{1}{1+\theta}}-\frac{1}{h[t]}, & \text { if } h[t] \geq \sum_{k=t}^{\mathcal{T}} \lambda[k] / \theta \\ 0, & \text { otherwise, }\end{cases}
$$

where $\lambda[k], k=1, \ldots, \mathcal{T}$ denote the Lagrange multipliers associated with constraint (4.36). Please note that when $\theta \rightarrow 0$, i.e., any amount of delay is allowed, then (4.37) provides the same solution as that obtained in [38] for fading channels.

3) Naive Scheme: In this naive scheme, in each transmission frame $t$, we assign $P[t]=$ $e[t]$, irrespective of the channel condition. Note that this scheme, being overly aggressive in spending energy, does not take into account the impact of channel and energy arrival statistics, and hence the long-term effect of the power allocation policy is completely ignored.

\subsection{Illustrative Results}

In this section, we evaluate the performances of the developed power control schemes and the baseline schemes for both average delay and delay-outage constraint models. We assume exponentially distributed channel power gain with an average value of $0 \mathrm{~dB}$. We assume a random energy profile that is uniformly distributed between 0 and $2 \bar{E}$ (Joule) [39]. Note that our developed scheme is general enough to be accommodated with any ergodic energy distribution. To incorporate the delay-outage constraint, we consider the maximum queue length, $Q^{\max }=8$ (bits) in Figs. 4.4 and 4.5. Further, the step-size parameters for learning rate sequences and for Lagrange multiplier update equations are chosen as $\phi[t]=(1 / t)^{0.70}$ 
and $\nu[t]=(1 / t)^{0.85}$, respectively.

\subsubsection{Average Delay Constraint}

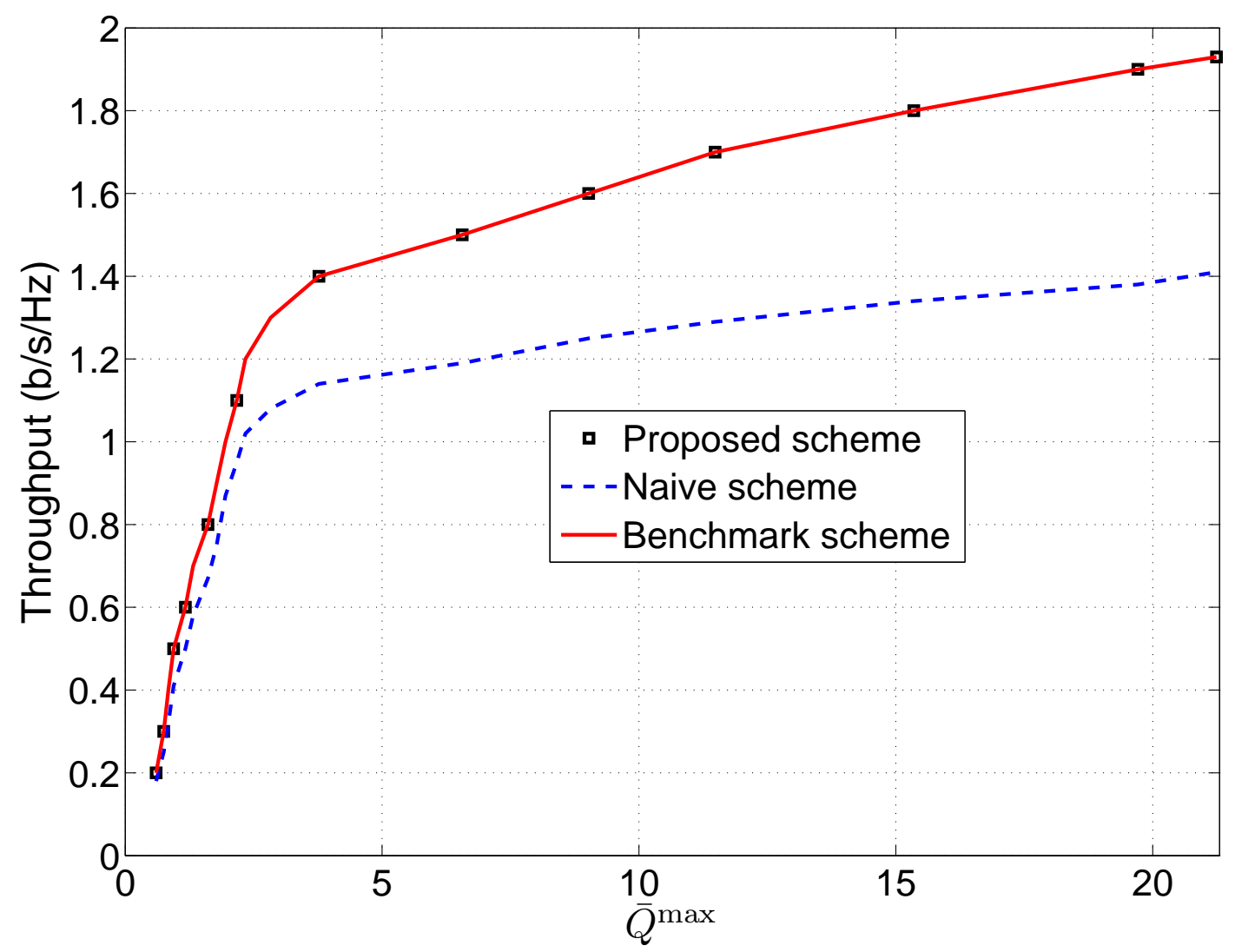

Fig. 4.2: Throughput versus average queue length $\bar{Q}^{\max }$.

Figure 4.2 shows the optimal supportable throughput versus queue length bound tradeoff curves for the proposed and baseline schemes under the average delay constraint model. We set the average $\mathrm{EH}$ rate $\bar{E}=2$, and evaluate the optimal throughputs for a given range of maximum time-averaged queue length $\bar{Q}^{\text {max }}$ (bits). We observe that the throughput increases with increasing queue length bound for both proposed and baseline schemes. However, the increasing rate of the throughput is high for smaller values of queue length bound, while the (increasing) rate slows down for higher values of the bound. Fig. 4.2 also shows that we achieve the same throughput for the proposed and the benchmark schemes. Recall that 


\section{Power Allocation with Energy Harvesting over Fading Channel under Statistical Delay Constraints}

the benchmark scheme for the average delay model does not keep track of the battery state in each time interval, and hence the control action taken in each time interval may not always be feasible. For instance, the calculated optimal power in a given time interval may be greater than the amount of remaining energy in the battery. Therefore, in spite of the lower computational complexity offered by the benchmark scheme, this scheme is not implementable in practice. In contrast, our developed scheme keeps track of both the battery and data queue length states, takes optimal control actions in each time interval, and still achieves the same optimal throughput as is achieved by the benchmark scheme. Further, we observe that the proposed scheme outperforms the naive scheme and the performance gap between the proposed and naive schemes increases with increasing queue length bound requirement. The naive scheme does not learn the channel and energy statistics over the transmission time and yields deteriorated performance by spending a large amount of energy that the battery contains in each time interval. Therefore, we can conclude that although the proposed scheme incurs higher complexity compared to the naive scheme, it is worth implementing the former scheme because of the large performance gap between the two schemes, particularly in the range of $\bar{Q}^{\max } \geq 3$.

In Fig. 4.3, we show throughput versus average harvested energy $\bar{E}$ for the proposed scheme for different values of the average queue length $\bar{Q}^{\text {max }}$. In particular, we consider $\bar{Q}^{\max }=\{0.75,1.5,2.5,4.5\}$. We observe that the throughput increases with increasing $\bar{E}$ for a given $\bar{Q}^{\text {max }}$. The higher available energy helps to transmit more data bits even when there is a stringent delay-requirement. However, the increasing rate of throughput is low for smaller values of $\bar{Q}^{\text {max }}$, whereas the (increasing) rate is high for higher values of $\bar{Q}^{\text {max }}$. For instance, increasing the average harvested energy $\bar{E}$ from 0.5 to 5 increases the throughput by 0.15 when $\bar{Q}^{\text {max }}=0.75$. On the other hand, with the same amount of incremental harvested energy, the throughput increases by 0.52 when $\bar{Q}^{\max }=4.5$.

\subsubsection{Delay-outage Constraint}

We compare the performance of our proposed scheme with that of the baseline schemes in Fig. 4.4 for $\bar{E}=2$. Note that similar to the conventional non-EH system [61], the effective capacity of a single link EH system increases with $\zeta_{Q}$. This result has already been shown in [73] for a single link EH system with known channel and energy profiles. Moreover, similar to Fig. 4.2, we observe that the proposed scheme provides the same optimal result 


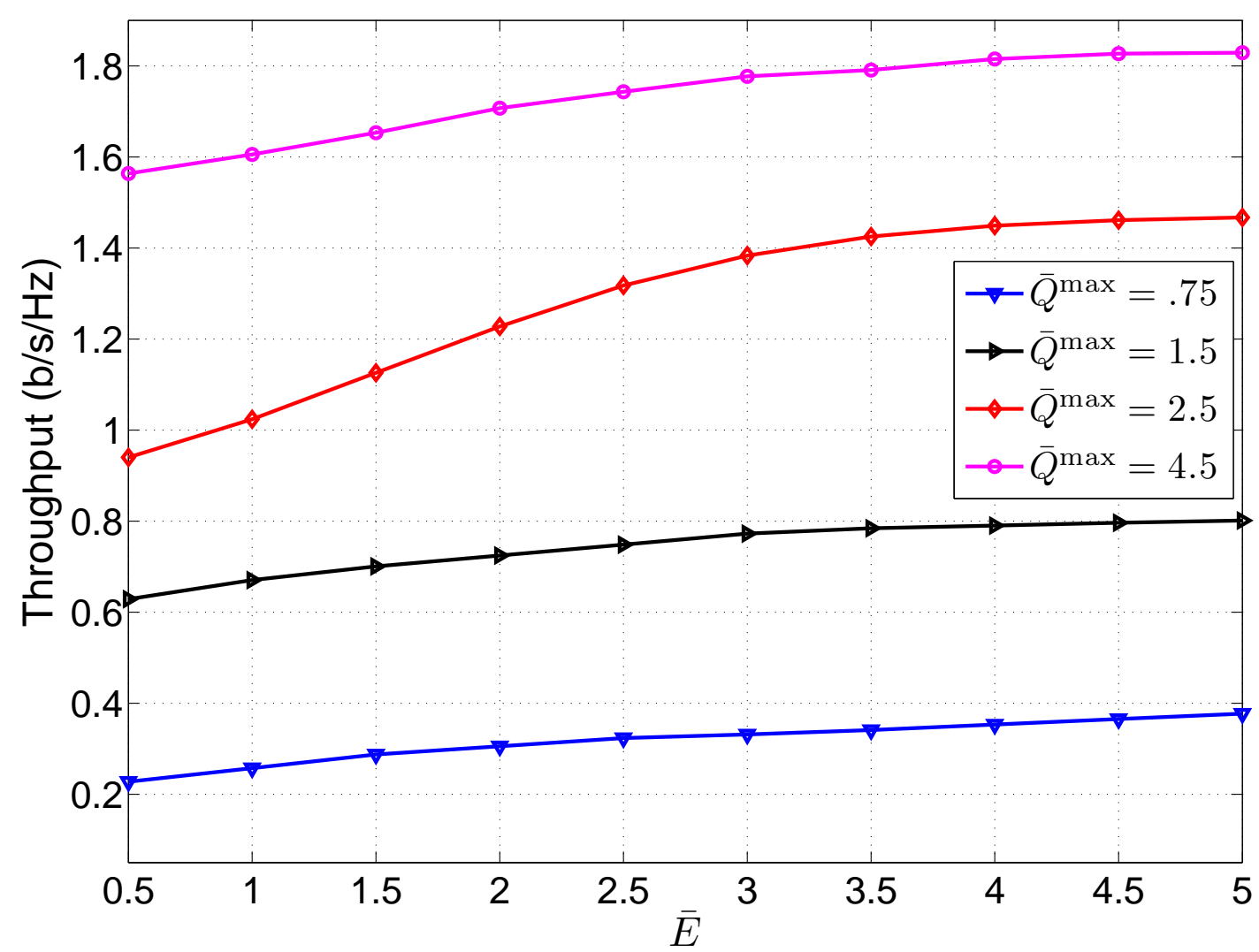

Fig. 4.3: Throughput versus average harvested energy $\bar{E}$.

as that obtained from the benchmark scheme for all the considered values of $\zeta_{Q}$. Therefore, we can conclude for the delay-outage constraint model that by considering the dynamics of the battery and applying the optimal control action according to the proposed learning algorithm, we can still achieve the same optimal effective capacity even for unknown channel and energy statistics. Moreover, the performance gap between our scheme and the naive baseline scheme for the considered range of $\zeta_{Q}$ exemplifies the impact of an intelligent power allocation strategy over a heuristic one, which does not take into account the channel and energy statistics to allocate the transmit power.

In Fig. 4.5, we show the behavior of the effective capacity with $\bar{E}$ for $\zeta_{Q}=\left\{10^{-6}, 10^{-4}, 10^{-2}\right.$, $\left.10^{-1}, 0.4,1\right\}$. We observe that the effective capacity increases with increasing $\bar{E}$ for a given queue-length-outage probability $\zeta_{Q}$. Note that $\zeta_{\mathrm{Q}, \mathrm{A}}$ denotes (simulated) outage probability 


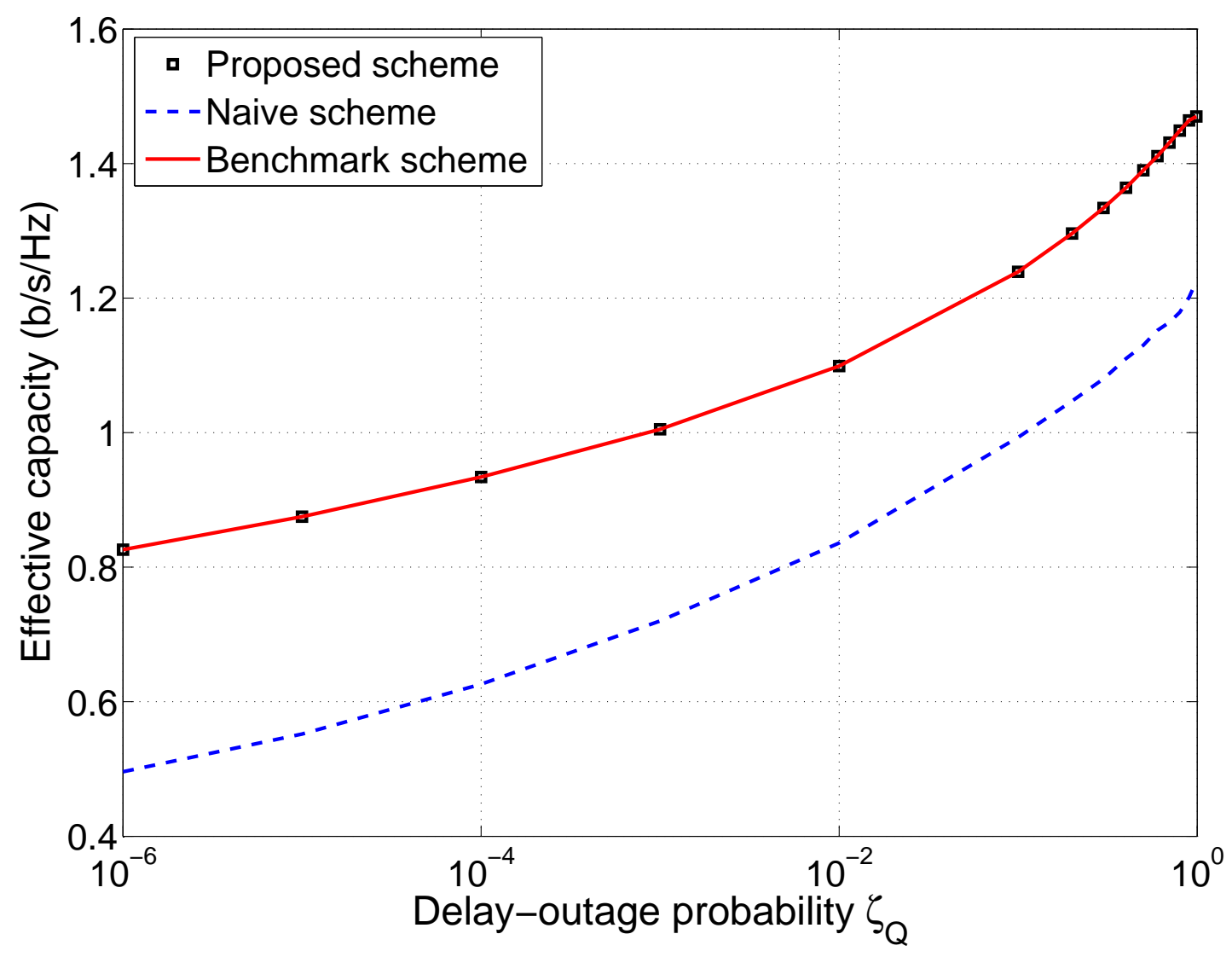

Fig. 4.4: Effective capacity versus $\zeta_{Q}$.

for average delay model; $\bar{Q}_{\mathrm{S}}$ denotes the (simulated) time-averaged queue length for delayoutage model. For instance, in case of $\zeta_{Q}=1$, i.e., unconstrained delay, the effective capacity can be increased by 1.53 if $\bar{E}$ is increased from 0.5 to 5 . However, the increasing rate of the effective capacity is comparatively less when there is a stringent delay constraint. For example, when $\zeta_{Q}=10^{-6}$, the effective capacity can be improved by 0.82 if we increase $\bar{E}$ from 0.5 to 5 . Furthermore, changing $\zeta_{Q}$ provides small impact on the effective capacity for small $\bar{E}$ (e.g., 0.31 of effective capacity is decreased when $\zeta_{Q}$ is changed from 1 to $10^{-6}$ for $\bar{E}=0.5$ ) and a larger variation in the effective capacity for large $\bar{E}$ (e.g., the effective capacity decreases by 1.02 when $\zeta_{Q}$ is changed from 1 to $10^{-6}$ for $\bar{E}=5$ ). 


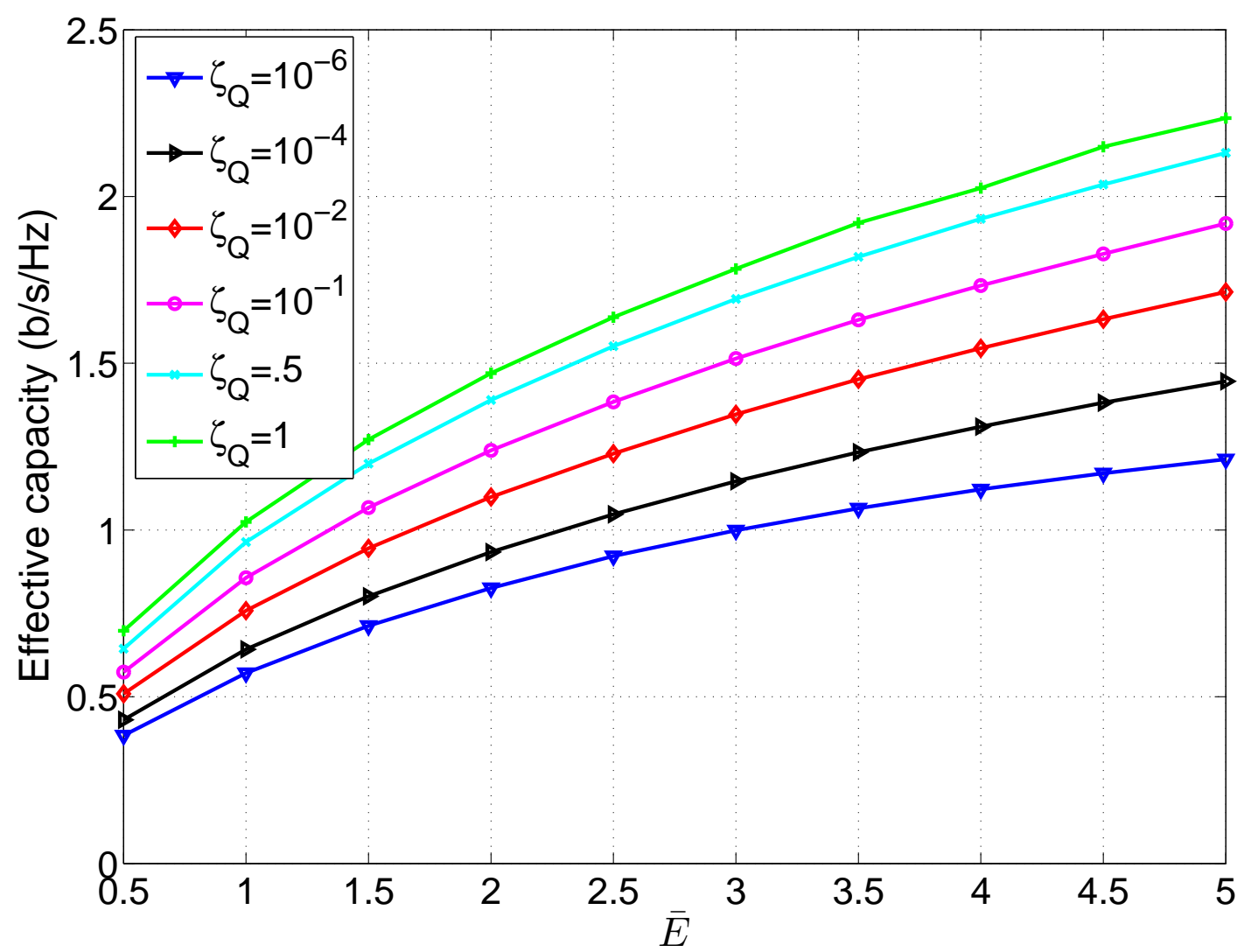

Fig. 4.5: Effective capacity versus average harvested energy $\bar{E}$.

\subsubsection{Average Delay versus Delay-outage Constraints}

We compare the performances of the average delay and delay-outage constraint models in Tables 4.1 and 4.2. We fix $Q^{\max }=8$ for both tables and consider two cases of queue-lengthoutage probabilities. Precisely, we set $\zeta_{Q}=0.1$ and $\zeta_{Q}=0.01$ for Table 4.1 and Table 4.2, respectively for the delay-outage constraint model. We vary $\bar{E}$ from 0.5 to 5 in steps of 0.5 and evaluate the effective capacity and the average queue length for each value of $\bar{E}$ of thedelay-outage constraint model. Then, we set the average queue length for the average delay model in such as a way so that we achieve the throughput same as the effective capacity obtained by the delay-outage constraint model. We calculate the outage probability for the average delay model by considering the events when the instantaneous queue length exceeds $Q^{\max }=8$ (the queue length bound for the delay-outage constraint model). By assuming 
Table 4.1: $\zeta_{Q}=0.1$.

\begin{tabular}{cccccc}
\hline $\bar{E}$ & $\zeta_{Q}$ & $\zeta_{\mathrm{Q}, \mathrm{A}}$ & $\mu$ & $\bar{Q}_{\mathrm{S}}$ & $\bar{Q}^{\mathrm{max}}$ \\
\hline \hline 0.5 & 0.1 & 0.19 & 0.56 & 2.07 & 1.55 \\
\hline 1.0 & 0.1 & 0.22 & 0.85 & 2.41 & 1.85 \\
\hline 1.5 & 0.1 & 0.23 & 1.06 & 2.72 & 2.13 \\
\hline 2.0 & 0.1 & 0.25 & 1.23 & 2.96 & 2.34 \\
\hline 2.5 & 0.1 & 0.26 & 1.38 & 3.10 & 2.49 \\
\hline 3.0 & 0.1 & 0.28 & 1.51 & 3.21 & 2.58 \\
\hline 3.5 & 0.1 & 0.29 & 1.63 & 3.35 & 2.65 \\
\hline 4.0 & 0.1 & 0.29 & 1.73 & 3.47 & 2.78 \\
\hline 4.5 & 0.1 & 0.3 & 1.81 & 3.55 & 2.88 \\
\hline 5.0 & 0.1 & 0.31 & 1.88 & 3.64 & 3.01 \\
\hline
\end{tabular}

Table 4.2: $\zeta_{Q}=0.01$

\begin{tabular}{cccccc}
$\overline{\bar{E}}$ & $\zeta_{\mathrm{Q}}$ & $\zeta_{\mathrm{Q}, \mathrm{A}}$ & $\mu$ & $\bar{Q}_{\mathrm{S}}$ & $\bar{Q}^{\mathrm{max}}$ \\
\hline \hline$\overline{0.5}$ & 0.01 & 0.0031 & 0.48 & 1.36 & 1.01 \\
\hline 1.0 & 0.01 & 0.0035 & 0.74 & 1.62 & 1.37 \\
\hline 1.5 & 0.01 & 0.0037 & 0.93 & 1.79 & 1.56 \\
\hline 2.0 & 0.01 & 0.0039 & 1.09 & 1.92 & 1.70 \\
\hline 2.5 & 0.01 & 0.0042 & 1.22 & 2.03 & 1.81 \\
\hline 3.0 & 0.01 & 0.0043 & 1.33 & 2.12 & 1.89 \\
\hline 3.5 & 0.01 & 0.0046 & 1.44 & 2.22 & 1.95 \\
\hline 4.0 & 0.01 & 0.0048 & 1.53 & 2.33 & 2.03 \\
\hline 4.5 & 0.01 & 0.0050 & 1.61 & 2.43 & 2.11 \\
\hline 5.0 & 0.01 & 0.0053 & 1.69 & 2.51 & 2.20 \\
\hline
\end{tabular}

$Q^{\max }=8$, we ensure that the maximum queue length is sufficiently long as compared to the average queue length in order to satisfy delay-outage constraint for all the considered values of $\bar{E}$. It is worth mentioning that we can show by KKT optimality conditions that the average queue length is same as the time-averaged queue length for the average delay model.

We observe that the average queue length for the delay-outage constraint model is higher than that for the average delay constraint model. In particular, the former is not intended to minimize the average queue length as opposed to the main objective of the latter. On the contrary, the latter yields higher queue-length-outage probability compared to the former. In the average delay constraint model, we cannot control the events, where the queue length 
exceeds a certain queue-threshold and hence we end up with a higher outage probability. Therefore, we can conclude that both average delay and delay-outage constraint models are important to be considered depending on the system applications. For instance, in case of real-time applications, where stringent delay outage probability is required, delay-outage constraint model is more appealing to be employed. On the contrary, in case of tight average delay requirement, average delay model is always a better choice.

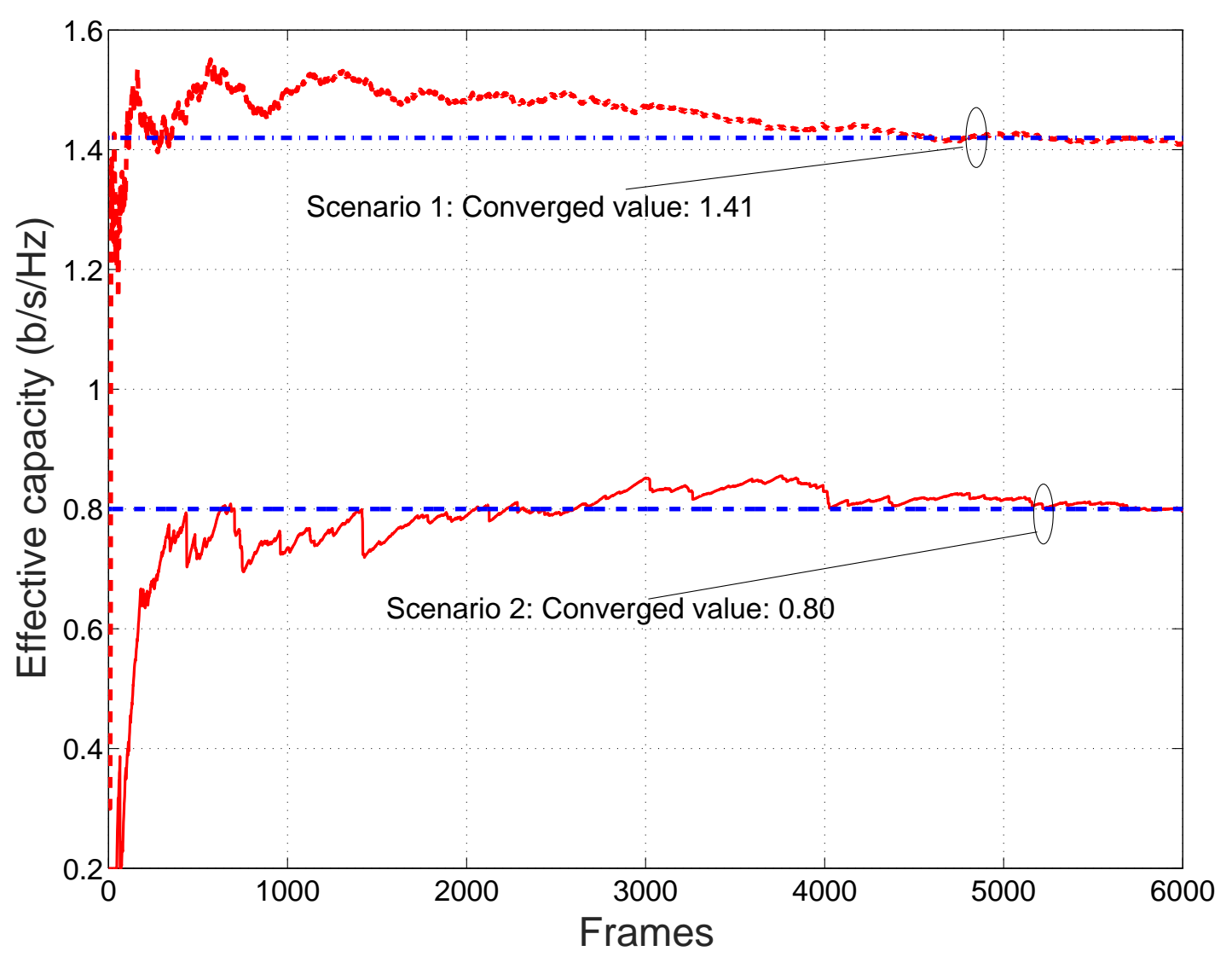

Fig. 4.6: Online allocation algorithm convergence: Effective capacity.

\subsubsection{Convergence Study of the Online Algorithms}

We show the convergence behavior of the proposed online power allocation algorithms. In order to avoid redundancy, we only show the results for the delay-outage constraint model. 


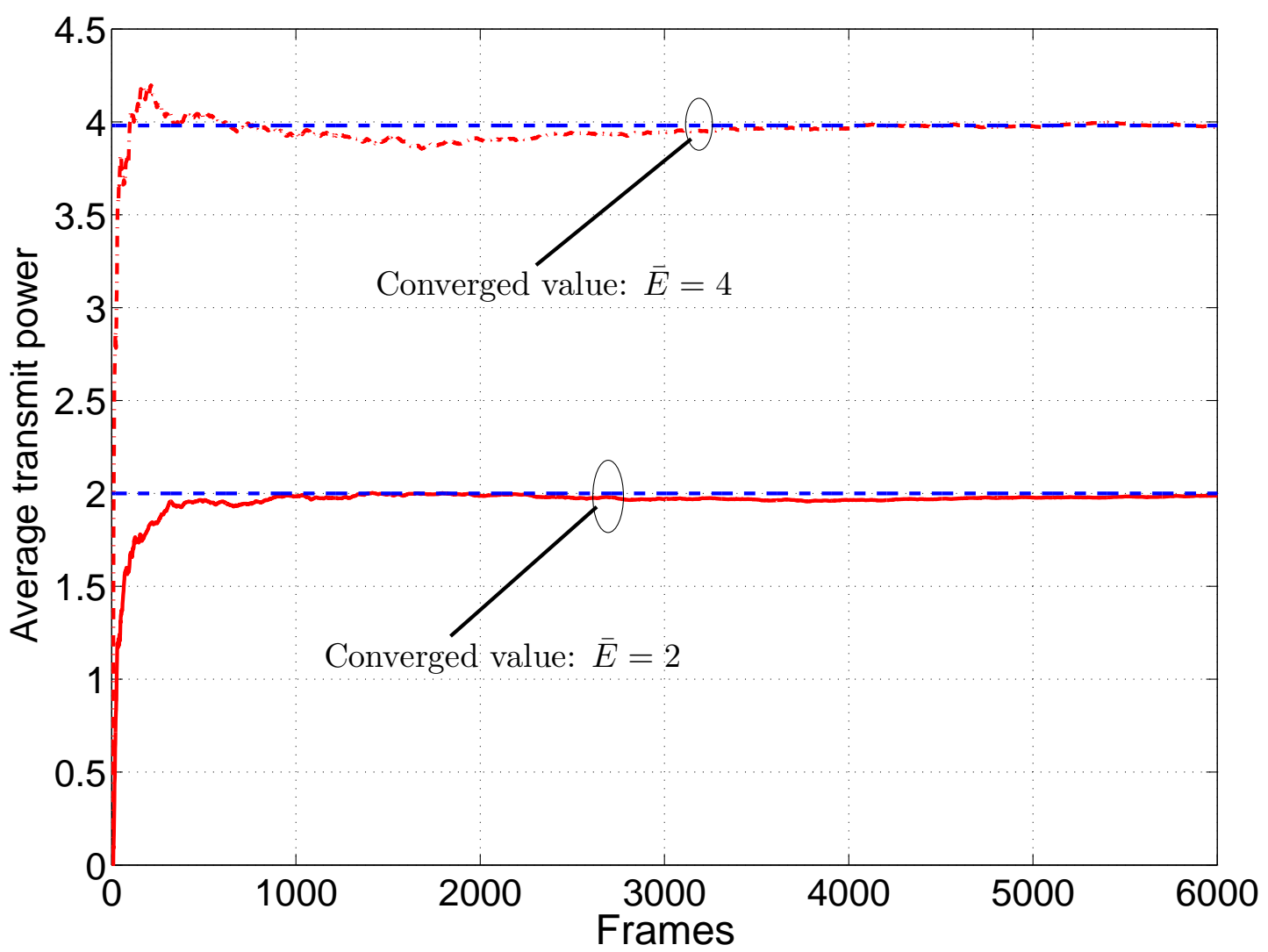

Fig. 4.7: Online allocation algorithm convergence: Average power.

In Fig. 4.6, we show the convergence of the effective capacity for the proposed learning algorithm for the delay-outage constraint model for two scenarios of queue-length-outage probability $\zeta_{Q}$. We assume $\zeta_{Q}=0.90$ and $\zeta_{Q}=10^{-6}$, respectively. Further, we adopt $\bar{E}=2$ and determine the running average parameter $R^{\mathrm{av}}[t]=\frac{t-1}{t} R^{\mathrm{av}}[t-1]+\frac{1}{t}(1+h[t] P[t])^{-\theta}$ to evaluate the effective capacity $R_{\mathrm{EC}}[t]$ in each time interval $t \geq 1$ as $R_{\mathrm{EC}}[t]=-\frac{1}{\theta \log (2)} \log R^{\mathrm{av}}[t]$. The results confirm that the proposed method converges to the optimal solution after 6000 transmission frames for both scenarios.

We further show the convergence of the average transmit power for two scenarios of the average harvested energy $\bar{E}$. We consider $\bar{E}=4$ and $\bar{E}=2$, respectively and assume $\zeta_{Q}=0.90$. Similar to Fig. 4.6, we evaluate the running average of the transmit power $P^{\text {av }}[t]$ in each time frame $t$ by $P^{\mathrm{av}}[t]=\frac{t-1}{t} P^{\mathrm{av}}[t-1]+\frac{1}{t} P[t], t \geq 1$. We observe that the average 
transmit powers converge after 6000 time frames for both scenarios. It is worth mentioning that the average transmit powers converge to the average harvested energy. This finding complies with the fact that the average transmit power for the proposed scheme achieves the same average value as that obtained from the benchmark scheme, because the constraint in (4.32) always meets with equality at the optimal point [105].

\subsection{Chapter Summary}

In this chapter, we have studied a point-to-point energy harvesting $(\mathrm{EH})$ communication system for two delay constraint models, namely the average delay and the delay-outage constraint models. For both delay models, we have maximized the supportable data arrival rate by ensuring the optimal control of transmit power over the transmission frames. For each model, we have formulated an Markov decision process (MDP) problem, adopted the postdecision state approach to efficiently exploit Bellman's equations, exploited the structural properties of the optimal policies, and developed online algorithms to be implemented in practice when the statistical knowledge of the underlying random channel fading and $\mathrm{EH}$ processes is unknown. Our developed algorithms are robust to the variations of system dynamics. We have derived optimal benchmark schemes (for both delay constraint models) that do not keep track of the battery dynamics. We have shown that our developed online algorithms, being practically implementable in contrast to the benchmark schemes, provide the same optimal throughput as that obtained by the benchmark schemes. Furthermore, we show by simulations that the average queue length of the average delay constraint model is less compared to that of the delay-outage constraint model. In contrast, the queue-lengthoutage probability for the latter is less than that for the former. 


\section{Chapter 5}

\section{Resource Allocation for Buffer-Aided Half-Duplex Relaying under Delay-Outage Constraint}

In Chapters 3 and 4, we have considered scenarios where the source communicates directly with the destination. When such direct communications is not possible, relaying communications is necessary to improve the coverage, throughput, and reliability of wireless networks. In this chapter, we focus on resource allocation for a dual-hop source-relay-destination bufferaided half-duplex (B-HD) relaying network over fading channels. To exploit the buffer-aided relaying capability, the B-HD relaying with adaptive link scheduling (B-HD-ALS) is proposed to efficiently schedule the source-relay link and relay-destination to be active in each frame depending on the instantaneous channel conditions.

This chapter investigates the optimal B-HD-ALS relaying under delay-outage constraint to maximize the constant supportable arrival rate $\mu$ to the source, (i.e., the effective capacity). In the considered model, the (end-to-end) delay is the summation of the delays at the source and relay buffers. Both cases of fixed and adaptive source and relay power allocation are investigated. In order to handle the delay-outage constraint, we need to know the tail distributions of the source and relay queue lengths, which are not tractable to derive in general. To overcome this difficulty, we assume large delay and employ asymptotic delay

Parts of Chapter 5 are presented at the 2014 IEEE International Conference on Communications (ICC) in London, UK [107], and published in the IEEE Transactions on Communications [108]. 
analysis to transform the delay-outage constraint into constraints on the minimum exponential decay rates (also called delay exponents) of the tail distributions. Next, the relationship between the delay exponents and resource allocation variables is derived. Consequently, we obtain a more tractable constrained optimization problem. We derive the link selection (or scheduling) and power allocation solutions as functions of the instantaneous link conditions and delay constraint using Lagrangian approach and convex optimization. Special cases on the link fading statistics and delay constraint are studied. Particularly, the proposed analysis is shown to converge to the existing results when the delay-outage constraint becomes very loose. Illustrative results are performed to demonstrate the effectiveness of the proposed QoS-aware B-HD-ALS relaying schemes over existing relaying schemes such as QoS-blind BHD-ALS [46], QoS-aware B-HD with fixed link scheduling (B-HD-FLS) [79], and non-buffer relaying [109] under different link conditions and delay constraints.

The rest of the chapter is organized as follows. In Section 5.1, the system model and the B-HD-ALS relaying problem are presented. Section 5.2 presents asymptotic delay analysis results for a buffer-aided relaying network. Sections 5.3 and 5.4 solve the B-HD-ALS relaying problems with fixed and adaptive power allocation, respectively. Illustrative results are provided in Section 5.5, followed by the Conclusions in Section 5.6.

\subsection{System Model and Problem Formulation}

\subsubsection{Model Description}

We consider a relaying network where a source $(\mathrm{S})$ communicates with a destination (D) with the help of a decode-and-forward relay $(\mathrm{R})$ using the same channel with bandwidth $B$ $(\mathrm{Hz})$ as shown in Fig. 5.1. We assume no direct communication link between the source and destination.

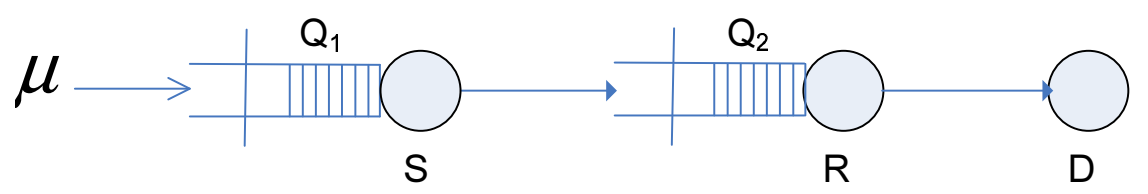

Fig. 5.1: B-HD relaying model.

We assume block-fading channels with fading duration equal to the transmission frame 


\section{Resource Allocation for Buffer-Aided Half-Duplex Relaying under Delay-Outage Constraint}

$T$ (seconds), i.e., the channel power gains remain unchanged during a frame but vary independently from frame to frame. Let $h_{1}[t]$, and $h_{2}[t]$ denote the normalized channel power gains of the S-R and R-D links in frame $t=1,2, \ldots$, respectively. Moreover, $h_{i}[t], i=1,2$ are assumed to be statistically independent with means $\mathbb{E}\left\{h_{i}\right\}$. Let $P_{1}$ and $P_{2}$ denote the power levels of the source and relay, respectively. The instantaneous signal-to-noise power ratio $\left(\mathrm{SNR}\right.$ ) values of the $\mathrm{S}-\mathrm{R}$ link and $\mathrm{R}-\mathrm{D}$ link in frame $t$ are thus $\mathrm{snr}_{1}[t]=P_{1} h_{1}[t]$, and $\operatorname{snr}_{2}[t]=P_{2} h_{2}[t]$, respectively. Using Shannon's formula, the corresponding instantaneous link transmission rates $(\mathrm{b} / \mathrm{s} / \mathrm{Hz})$ if active are:

$$
r_{i}[t]=\log _{2}\left(1+\operatorname{snr}_{i}[t]\right), i=1,2
$$

The average link SNR values are $\mathrm{SNR}_{i}=\mathbb{E}\left\{\operatorname{snr}_{i}[t]\right\}, i=1,2$.

In this work, we consider the B-HD-ALS relaying problem described as follows. Let $\phi[t] \in\{0,1\}, \forall t$ denote a binary variable for frame $t$ where we set $\phi[t]=1$ if the R-D link is active and $\phi[t]=0$ if the S-R link is active. One way to implement the adaptive relaying scheme is that the relay makes the link selection decision and informs the source, for example, by using one-bit feedback. We next describe the objective and the constraint for the link selection problem.

As shown in Fig. 5.1, the source utilizes its buffer to store the arriving traffic with the constant rate $\mu$ (in $\mathrm{b} / \mathrm{s} / \mathrm{Hz}$ ). The relay also employs its own buffer to store the received data from the source before transmitting to the destination. Using the above notations, the service processes of the source and the relay queues are $\left\{(1-\phi[t]) T B r_{1}[t]\right\}$, and $\left\{\phi[t] T B r_{2}[t]\right\}$, respectively.

Denote $q_{1}[t], q_{2}[t] \geq 0$ as the queue lengths of the source and relay buffers, respectively, in frame $t=1,2, \ldots$. Then, the corresponding queue-length dynamics are given as:

$$
\begin{aligned}
& q_{1}[t+1]=q_{1}[t]-\min \left\{q_{1}[t],(1-\phi[t]) T B r_{1}[t]\right\}+T B \mu, \\
& q_{2}[t+1]=q_{2}[t]+\min \left\{q_{1}[t],(1-\phi[t]) T B r_{1}[t]\right\}-\min \left\{q_{2}[t], \phi[t] T B r_{2}[t]\right\}
\end{aligned}
$$

where we account for the fact that the maximal number of bits transmitted from the source (or the relay) is limited by the number of bits available in the source (or the relay) buffer and the instantaneous capacity of the S-R link (or the R-D link). The last term of the second equation represents the actual amount of data arriving at the destination. 
Assume stable source and relay queues, i.e., the queue lengths $q_{1}[t]$ and $q_{2}[t]$ do not grow unboundedly large as $t \rightarrow \infty$, and hence, having steady-state distributions. Denote the steady-state queue length random variables as $Q_{1}$ and $Q_{2}$. In this work, we consider the queue-length-outage constraint on the maximum acceptable E2E queue length $Q=Q_{1}+Q_{2}$, which can be expressed as:

$$
\operatorname{Pr}\left(Q>Q^{\max }\right) \leq \zeta_{Q}
$$

\subsubsection{Problem Formulation}

We formulate the optimal B-HD-ALS relaying design problem to maximize the supportable rate $\mu$ to the source under the queue-length-outage constraint as follows:

$$
\max _{\mu, \phi[t] \in\{0,1\}} \mu \quad \text { s.t.: Constraint (5.2). }
$$

The optimal value of (5.3) is called the effective capacity of the B-HD-ALS relaying.

It can be seen that, to solve problem (5.3), we need to know the tail distribution of $Q$, which is very difficult to obtain in general. One way to circumvent this problem is to consider the large queue length (or delay) region and then employ the asymptotic delay analysis to attain the tail distribution of $Q$ [34]. Hence, in the remaining of this paper, $Q^{\text {max }}$ is assumed to be sufficiently large (but finite).

We next show how to transform the queue-length-outage constraint (5.2) into more tractable constraints.

\subsection{Delay-Outage Constraint Transformation}

\subsubsection{Asymptotic Delay Analysis for Buffer-aided Relaying Network}

Assume that the optimal relaying scheme $\phi^{*}[t]$ of (5.3) achieves the delay exponents $\theta_{1}$, and $\theta_{2}$ at the source and relay queues, respectively. It implies that for sufficiently large $x$, from (2.3), we have the following tail distributions of the queue lengths:

$$
\operatorname{Pr}\left(Q_{1}>x\right)=e^{-\theta_{1} x}, \quad \operatorname{Pr}\left(Q_{2}>x\right)=e^{-\theta_{2} x}
$$




\section{Resource Allocation for Buffer-Aided Half-Duplex Relaying under Delay-Outage Constraint}

The probability density functions (pdfs) of the corresponding queue lengths are given by:

$$
f_{Q_{1}}(x)=\theta_{1} e^{-\theta_{1} x}, \quad f_{Q_{2}}(x)=\theta_{2} e^{-\theta_{2} x}
$$

We can see that the relaying scheme $\phi^{*}[t]$ must attain the constraint (5.2) with equality; otherwise, we can always increase the supportable arrival rate without violating the delay constraint. On the other hand, for a given queue length bound, the optimal relaying scheme $\phi^{*}[t]$ with $\theta_{1}$ and $\theta_{2}$ must achieve the smallest outage probability.

We can derive the queue length $Q=Q_{1}+Q_{2}$ outage probability as follows [110]:

$$
\begin{aligned}
\operatorname{Pr}(Q>x)=1-\operatorname{Pr}\left(Q_{1}+Q_{2} \leq x\right) & =1-\int_{0}^{x} f_{Q_{1}}(y) \int_{0}^{x-y} f_{Q_{2}}(z) d z d y \\
& =\frac{\theta_{1} e^{-\theta_{2} x}-\theta_{2} e^{-\theta_{1} x}}{\theta_{1}-\theta_{2}}=\left[\frac{e^{\theta_{\Delta} x}-1}{\theta_{\Delta}} \theta_{M}+1\right] e^{-\theta_{M} x}
\end{aligned}
$$

where $\theta_{M}=\max \left\{\theta_{1}, \theta_{2}\right\}, 0 \leq \theta_{\Delta}=\max \left\{\theta_{1}, \theta_{2}\right\}-\min \left\{\theta_{1}, \theta_{2}\right\}<\theta_{M}$. For the assumed sufficiently large $x \gg 1$, as $\theta_{\Delta}$ decreases, the term $\left(e^{\theta_{\Delta} x}-1\right) / \theta_{\Delta}$ decreases and reaches the smallest value at $\theta_{\Delta}=0$ (i.e., $\left.\theta_{1}=\theta_{2}\right)$, which is $\lim _{\theta_{\Delta} \rightarrow 0}\left(e^{\theta_{\Delta} x}-1\right) / \theta_{\Delta}=x$ by using L'Hopital rule. In other words, the optimal relaying scheme $\phi^{*}[t]$ must achieve $\theta_{1}=\theta_{2}$ to obtain the smallest outage probability $\operatorname{Pr}(Q>x)=\left(1+\theta_{1} x\right) e^{-\theta_{1} x}$. Hence, the constraint (5.2) becomes: $\operatorname{Pr}\left(Q>Q^{\max }\right)=\left(1+\theta_{1} Q^{\max }\right) e^{-\theta_{1} Q^{\max }}=\zeta_{Q}$. The delay exponents can be computed as:

$$
\theta_{1}=\theta_{2}=\theta^{\mathrm{tar}} \triangleq-\frac{1}{Q^{\max }}\left(1+\mathcal{W}_{-1}\left(-\frac{\zeta_{Q}}{e}\right)\right)
$$

where $\mathcal{W}_{-1}($.$) denotes the lower branch of the real-valued Lambert \mathrm{W}$ function [111], and the Lambert $\mathrm{W}$ function is the inverse of $Z(W)=W e^{W}$.

Note that by applying the Little's law to the considered source and relay queues in tandem and using the tail distribution of the end-to-end queue-length $Q$, we can express the tail distribution of the end-to-end delay $D$ (in seconds) with $\theta_{1}=\theta_{2}=\theta^{\text {tar }}$ as follows:

$$
\operatorname{Pr}(D>x)=\operatorname{Pr}\left(Q>x \mu^{*} B\right)=\left(1+x \mu^{*} B \theta^{\operatorname{tar}}\right) e^{-x \mu^{*} B \theta^{\operatorname{tar}}},
$$

where $\mu^{*}$ is the optimal value of (5.3). 


\subsubsection{Delay-outage Constraint Transformation}

By applying condition (2.2) at the source and relay queues, from the previous analysis, we can see that in order to obtain the largest supportable arrival rate $\mu$ while satisfying the delay constraint (5.2), the following conditions must be satisfied:

$$
\begin{aligned}
T B \mu \theta_{1}+\Omega_{1}\left(-\theta_{1}\right) & =0 \\
\Omega_{2}^{\operatorname{arv}}\left(\theta_{2}\right)+\Omega_{2}\left(-\theta_{2}\right) & =0 \\
\theta_{1}=\theta_{2} & =\theta^{\text {tar }}
\end{aligned}
$$

where $\Omega_{1}(\theta)$ and $\Omega_{2}(\theta)$ are the log moment generating functions (LMGFs) of the service processes of the source and relay queues, i.e.,:

$$
\Omega_{1}(\theta)=\log \mathbb{E}\left\{e^{\theta(1-\phi[t]) T B r_{1}[t]}\right\}, \quad \Omega_{2}(\theta)=\log \mathbb{E}\left\{e^{\theta \phi[t] T B r_{2}[t]}\right\},
$$

where $r_{1}[t]$ and $r_{2}[t]$ are given by (5.1). $\Omega_{2}^{\text {arv }}\left(\theta_{2}\right)$ is the LMGF of the arrival process to the relay which is the same as the LMGF of the the departure process of the source and is given by [60, Example 2.5]:

$$
\Omega_{2}^{\operatorname{arv}}(\theta)= \begin{cases}T B \mu \theta, & 0 \leq \theta \leq \theta_{1} \\ T B \mu \theta_{1}+\Omega_{1}\left(\theta-\theta_{1}\right), & \theta>\theta_{1}\end{cases}
$$

From (5.6) and (5.8), we have: $\Omega_{2}^{\operatorname{arv}}(\theta)=T B \mu \theta$.

We can see that the delay-outage constraint (5.2) can be transformed into the following equivalent constraints in terms of arrival rate $\mu$ and link selection variables:

$$
\mu T B \theta^{\mathrm{tar}}+\Omega_{i}\left(-\theta^{\mathrm{tar}}\right)=0, i=1,2 .
$$

\subsection{Adaptive Link Scheduling with Fixed Power Allocation}

\subsubsection{Optimal Solution}

Using (5.9), the problem (5.3) can be now re-formulated as:

$$
\max _{\mu, \phi[t] \in\{0,1\}} \quad \mu \quad \text { s.t.: } T B \mu \theta^{\text {tar }}+\Omega_{i}\left(-\theta^{\text {tar }}\right)=0, i=1,2 .
$$




\section{Resource Allocation for Buffer-Aided Half-Duplex Relaying under Delay-Outage Constraint}

The problem (5.10) involves link selection variables whose solutions are studied next.

Using the expressions in (5.7) and the increasing monotonicity of the log function, after some simple manipulations, the problem (5.10) can be re-expressed as follows:

$$
\min _{\phi[t] \in\{0,1\}} \mathbb{E}\left\{e^{-\theta^{\operatorname{tar}}(1-\phi[t]) T B r_{1}[t]}\right\} \quad \text { s.t. : } \quad \mathbb{E}\left\{e^{-\theta^{\operatorname{tar}}(1-\phi[t]) T B r_{1}[t]}\right\}=\mathbb{E}\left\{e^{-\theta^{\operatorname{tar}} \phi[t] T B r_{2}[t]}\right\} .
$$

We can see that the objective function and the left-hand side of the equality constraint increase while the right-hand side decreases with increasing $\phi[t]$. Hence, we can replace the equality constraint in (5.11) by the greater-than-or-equal inequality constraint without loosing optimality because the inequality constraint must be met with equality under optimal solution $\phi^{*}[t]$. Otherwise, we can always find another feasible solution with smaller objective value. In other words, the problem (5.11) can be expressed as:

$$
\min _{\phi[t] \in\{0,1\}} \mathbb{E}\left\{e^{-\theta(1-\phi[t]) r_{1}[t]}\right\} \quad \text { s.t. : } \mathbb{E}\left\{e^{-\theta(1-\phi[t]) r_{1}[t]}\right\} \geq \mathbb{E}\left\{e^{-\theta \phi[t] r_{2}[t]}\right\}, \theta \triangleq T B \theta^{\operatorname{tar}}
$$

The optimal value of (5.3) or the effective capacity is:

$$
\mu_{\mathrm{B}-\mathrm{HD}-\mathrm{ALS}}=-\log \mathbb{E}\left\{e^{-\theta\left(1-\phi^{*}[t]\right) r_{1}[t]}\right\} / \theta
$$

We can observe that under more stringent delay constraints, i.e., larger $\theta$, the effective capacity is smaller and approaches zero as $\theta$ tends to infinity.

To solve problem (5.12), we employ the Lagrangian approach [46], [81], [112], [113]. Toward this end, for ease of understanding, we re-write the optimization problem (5.12) for $\mathcal{T} \rightarrow \infty$ as follows:

$$
\min _{\phi[t] \in\{0,1\}} \frac{1}{\mathcal{T}} \sum_{t=1}^{\mathcal{T}} e^{-\theta(1-\phi[t]) r_{1}[t]} \quad \text { s.t. }: \quad \frac{1}{\mathcal{T}} \sum_{t=1}^{\mathcal{T}} e^{-\theta(1-\phi[t]) r_{1}[t]} \geq \frac{1}{\mathcal{T}} \sum_{t=1}^{\mathcal{T}} e^{-\theta \phi[t] r_{2}[t]} .
$$

The equivalent Lagrangian function of problem (5.14) can be written as follows:

$$
\mathcal{L}(\lambda, \phi)=\frac{1}{\mathcal{T}} \sum_{t=1}^{\mathcal{T}}\left[(1-\lambda) e^{-\theta(1-\phi[t]) r_{1}[t]}+\lambda e^{-\theta \phi[t] r_{2}[t]}\right]
$$

where $\boldsymbol{\phi}=(\phi[1], \ldots, \phi[\mathcal{T}])$ denotes the vector of (binary) link selection variables; $\lambda$ is non- 
negative Lagrange multiplier associated with the inequality constraint in (5.14). Note that if we are to minimize $\mathcal{L}(\lambda, \phi)$ with respect to $\phi$ for a given $\lambda$, and $\lambda$ is determined to satisfy the constraint in (5.14) with equality at optimality, we will obtain the optimal solution of (5.14) as follows [113].

First, we need to determine the optimal solution $\phi^{*}=\left(\phi^{*}[1], \ldots, \phi^{*}[\mathcal{T}]\right)$ for a given value of $\lambda$ to minimize the Lagrangian, i.e.,:

$$
\min _{\phi[t] \in\{0,1\}, t=1, \ldots, \mathcal{T}} \mathcal{L}(\lambda, \phi)
$$

By carefully studying the Lagrangian function (5.15), we can see that the optimization problem (5.16) can be decomposed into $\mathcal{T}$ sub-problems, each for one particular frame $t=$ $1, \ldots, \mathcal{T}$ as:

$$
\min _{\phi[t] \in\{0,1\}}(1-\lambda) e^{-\theta(1-\phi[t]) r_{1}[t]}+\lambda e^{-\theta \phi[t] r_{2}[t]} .
$$

By computing the objective function value at $\phi[t]=0$ or 1 , the optimal link selection solution $\phi^{*}[t]$ in frame $t$ can be easily obtained as:

$$
\phi^{*}[t]= \begin{cases}0, & (1-\lambda) e^{-\theta r_{1}[t]}+\lambda \leq(1-\lambda)+\lambda e^{-\theta r_{2}[t]} \\ 1, & \text { otherwise. }\end{cases}
$$

Note that, in (5.18), breaking ties is randomly performed, i.e., the S-R and R-D links are selected with equal probability 0.5 . It must hold true that $\lambda \in(0,1)$. Otherwise, we would have trivial solution $\phi^{*}[t]=1$ (for $\lambda=0$ ) or 0 (for $\lambda \geq 1$ ), $\forall t$.

Second, the multiplier $\lambda$ is determined such that $\phi^{*}$ satisfies (5.14) with equality. In general, a closed-form solution for $\lambda$ does not exist, however, we can use numerical search to find $\lambda$.

For further analysis, we define $\xi=\lambda /(1-\lambda)>0$, and have the following cases:

(i) For $\xi \in(0,1)$, the link selection solution (5.18) can be expressed as:

$$
\phi^{*}[t]= \begin{cases}0, & r_{1}[t] \geq-\frac{1}{\theta} \log \left(1+\xi\left(e^{-\theta r_{2}[t]}-1\right)\right), \\ 1, & \text { otherwise. }\end{cases}
$$


(ii) For $\xi=1$, the link selection solution (5.18) can be expressed as:

$$
\phi^{*}[t]= \begin{cases}0, & r_{1}[t] \geq r_{2}[t] \\ 1, & \text { otherwise }\end{cases}
$$

(iii) For $\xi \in(1, \infty)$, the link selection solution (5.18) can be expressed as:

$$
\phi^{*}[t]= \begin{cases}0, & r_{2}[t] \leq-\frac{1}{\theta} \log \left(1+\xi^{-1}\left(e^{-\theta r_{1}[t]}-1\right)\right) \\ 1, & \text { otherwise }\end{cases}
$$

For each of the above three cases, the multiplier $\xi$ can be determined so that the inequality constraint in (5.12) holds with equality. Hence, $\xi$ captures the statistical information on the link fading and SNR values. The link selection exploits the fading diversity. In case (ii) with $\xi=1$, the link selection depends only on the instantaneous link rates. However, in both cases (i) and (iii), the link selection depends on the instantaneous link rates as well as the delay exponent $\theta$. For example, more insights into the link selection solution can be revealed by considering the case (i) with $\xi \in(0,1)$. When $r_{2}[t]$ is sufficiently small, $-\frac{1}{\theta} \log \left(1+\xi\left(e^{-\theta r_{2}[t]}-1\right)\right)$ approaches 0 , and the S-R link is often selected. Moreover, when $r_{2}[t]$ is very large, it approaches a strictly positive constant value, namely $-\log (1-\xi) / \theta$. Hence, only when $r_{1}$ is larger than this value, the S-R link is selected.

\subsubsection{Special Cases}

\subsubsection{Case of very loose delay constraints}

When $\theta$ approaches 0 , using L'Hopital rule, we can derive the following limits:

$$
\begin{gathered}
\lim _{\theta \rightarrow 0}-\frac{1}{\theta} \log \left(1+\xi\left(e^{-\theta r_{2}[t]}-1\right)\right)=\lim _{\theta \rightarrow 0} \frac{\xi r_{2}[t] e^{-\theta r_{2}[t]}}{1+\xi\left(e^{-\theta r_{2}[t]}-1\right)}=\xi r_{2}[t] \\
\lim _{\theta \rightarrow 0}-\frac{1}{\theta} \log \left(1+\xi^{-1}\left(e^{-\theta r_{1}[t]}-1\right)\right)=\xi^{-1} r_{1}[t]
\end{gathered}
$$

for the cases $\xi \in(0,1)$, and $\xi \in(1, \infty)$, respectively. 
Hence, in this case, the link selection solutions (5.19) and (5.21) converge to the following general form:

$$
\phi^{*}[t]= \begin{cases}0, & r_{1}[t] \geq \xi r_{2}[t] \\ 1, & \text { otherwise }\end{cases}
$$

for $\xi \in(0, \infty)$. In addition, this solution satisfies the following condition:

$$
\lim _{\theta \rightarrow 0}-\frac{1}{\theta} \mathbb{E}\left\{e^{-\theta\left(1-\phi^{*}[t]\right) r_{1}[t]}\right\}=\lim _{\theta \rightarrow 0}-\frac{1}{\theta} \mathbb{E}\left\{e^{-\theta \phi^{*}[t] r_{2}[t]}\right\}
$$

Again, by applying the L'Hopital rule for both sides, the above condition becomes:

$$
\mathbb{E}\left\{\left(1-\phi^{*}[t]\right) r_{1}[t]\right\}=\mathbb{E}\left\{\phi^{*}[t] r_{2}[t]\right\}
$$

The link selection solution (5.22) was derived in [46] under a-priori unconstrained delay assumption, e.g., see Section 2.4.1.1. Hence, the our analysis with delay-outage constraint contains the unconstrained delay assumption as a special case.

\subsubsection{Case of very stringent delay constraints}

When $\theta$ approaches $\infty$ (as the outage probability $\zeta_{Q}$ approaches 0 ), $\xi=1$ and the link selection solution (5.20) applies. However, as mentioned previously, under very stringent delay constraints, the effective capacity approaches 0 .

\subsubsection{Case of negligible fading variation}

When the R-D link has an almost constant capacity due to negligible fading variation $r_{2}[t]=$ $R_{2}, \forall t$, the link selection solution (5.19) is used with $r_{2}[t]=R_{2}$ and $\xi<1$. As a result, the S-R link is selected when its instantaneous rate $r_{1}[t]$ is larger than a (fixed) threshold, otherwise, the R-D link is selected. Similarly, when the S-R link has negligible fading variation with an almost constant rate $r_{1}[t]=R_{1}, \forall t$, the link selection solution (5.21) applies with $r_{1}[t]=R_{1}$ and $\xi>1$.

\subsubsection{Case of similar link fading distributions}

When both the S-R and R-D links have similar fading distributions, whether $\xi$ is less than, equal to, or larger than 1 depends only on the average link SNR values $\mathrm{SNR}_{1}$ and $\mathrm{SNR}_{2}$. 
If $\mathrm{SNR}_{1}=\mathrm{SNR}_{2}$, then $\xi=1$, and the link selection solution (5.20) applies. If $\mathrm{SNR}_{1}<$ $\mathrm{SNR}_{2}$, then $\xi$ increases with increasing $\theta$, approaches 1 for sufficiently large $\theta$, and the link selection solution (5.19) applies. If $\mathrm{SNR}_{1}>\mathrm{SNR}_{2}$, then $\xi$ decreases with increasing $\theta$, approaches 1 for sufficiently large $\theta$, and the link selection solution (5.21) applies.

The above results can be used to devise a numerical search algorithm for the multiplier $\xi$ as follows.

For $x, \psi>0$, and $\hat{\theta}=\theta / \log (2)$, define: $r(x)=2^{x}-1$, and $f(x, \psi)=-\log (1+\psi((1+$ $\left.\left.x)^{-\hat{\theta}}-1\right)\right) /(\hat{\theta} \log (2))$.

We first consider case (i) with $\xi \in(0,1)$. Then, the link selection solution (5.19) can be expressed in terms of the link instantaneous SNR values as follows:

$$
\phi^{*}[t]= \begin{cases}0, & \operatorname{snr}_{1}[t] \geq r\left(f\left(\operatorname{snr}_{2}[t], \xi\right)\right) \\ 1, & \text { otherwise }\end{cases}
$$

Hence, we can compute the expectation terms in (5.12) as:

$$
\begin{aligned}
\mathbb{E}\left\{e^{-\theta\left(1-\phi^{*}[t]\right) r_{1}[t]}\right\}= & \int_{0}^{\infty}\left[\left[\int_{0}^{r\left(f\left(x_{2}, \xi\right)\right)} 1+\int_{r\left(f\left(x_{2}, \xi\right)\right)}^{\infty}\left(1+x_{1}\right)^{-\hat{\theta}}\right] f_{\mathrm{snr}_{1}}\left(x_{1}\right) d x_{1}\right] f_{\mathrm{snr}_{2}}\left(x_{2}\right) d x_{2} \\
\mathbb{E}\left\{e^{-\theta \phi^{*}[t] r_{2}[t]}\right\}= & \int_{0}^{r(-\log (1-\xi) / \theta)}\left[\left[\int_{0}^{r\left(f\left(x_{1}, \xi^{-1}\right)\right)} 1+\int_{r\left(f\left(x_{1}, \xi^{-1}\right)\right)}^{\infty}\left(1+x_{2}\right)^{-\hat{\theta}}\right] f_{\text {snr } \left._{2}\left(x_{2}\right) d x_{2}\right]} f_{\operatorname{snr}_{1}\left(x_{1}\right) d x_{1}}\right.
\end{aligned}
$$

where $f_{\mathrm{snr}_{1}}\left(x_{1}\right)$ and $f_{\mathrm{snr}_{2}}\left(x_{2}\right)$ denote the probability density functions of $\operatorname{snr}_{1}[\mathrm{t}]$ and $\mathrm{snr}_{2}[\mathrm{t}]$.

Notice that the terms increase in (5.26), and decrease in (5.27) with increasing $\xi$. Hence, as described in Algorithm 1, a simple one-dimension bisection search over $\xi \in(0,1)$ can be carried out to determine $\xi$ in which the terms in (5.26), (5.27) are equal. Such value can be computed offline since these terms involve only statistical properties of the S-R and R-D links. 


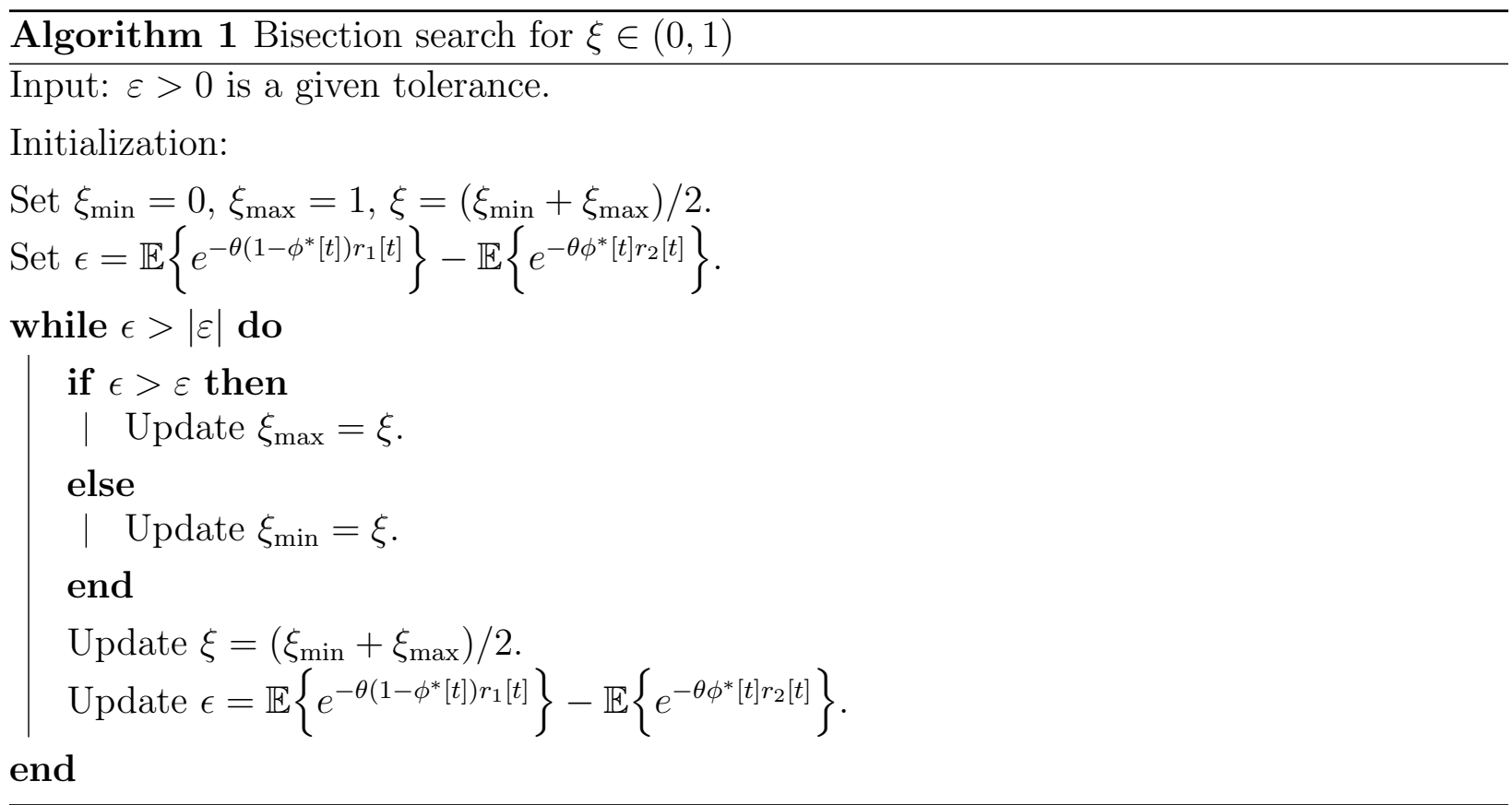

We now consider case (iii) with $\xi \in(1, \infty)$. The link selection solution (5.21) can be expressed in terms of the link instantaneous SNRs as:

$$
\phi^{*}[t]= \begin{cases}0, & \operatorname{snr}_{2}[t] \leq r\left(f\left(\operatorname{snr}_{1}[t], \xi^{-1}\right)\right) \\ 1, & \text { otherwise }\end{cases}
$$

When $\operatorname{snr}_{1}[t]$ becomes very large, $r\left(f\left(\operatorname{snr}_{1}[t], \xi^{-1}\right)\right)$ approaches a strictly positive constant value $r\left(-\log \left(1-\xi^{-1}\right) / \theta\right)$. We can compute the expectation terms in (5.12) as follows.

$$
\begin{aligned}
& \mathbb{E}\left\{e^{-\theta\left(1-\phi^{*}[t]\right) r_{1}[t]}\right\}= \int_{0}^{r\left(-\log \left(1-\xi^{-1}\right) / \theta\right)}\left[\left[\int_{0}^{r\left(f\left(x_{2}, \xi\right)\right)} 1+\int_{r\left(f\left(x_{2}, \xi\right)\right)}^{\infty}\left(1+x_{1}\right)^{-\hat{\theta}}\right] f_{\mathrm{snr}_{1}}\left(x_{1}\right) d x_{1}\right] \\
& f_{\mathrm{snr}_{2}\left(x_{2}\right) d x_{2}+\int_{r\left(-\log \left(1-\xi^{-1}\right) / \theta\right)}^{\infty} f_{\mathrm{snr}_{2}}\left(x_{2}\right) d x_{2},} \\
& \mathbb{E}\left\{e^{-\theta \phi^{*}[t] r_{2}[t]}\right\}=\int_{0}^{\infty}\left[\left[\int_{0}^{r\left(f\left(x_{1}, \xi^{-1}\right)\right)} 1+\int_{r\left(f\left(x_{1}, \xi^{-1}\right)\right)}^{\infty}\left(1+x_{2}\right)^{-\hat{\theta}}\right] f_{\mathrm{snr}_{2}}\left(x_{2}\right) d x_{2}\right] f_{\mathrm{snr}_{1}}\left(x_{1}\right) d x_{1} .
\end{aligned}
$$

Similarly, we can find $\xi$ such that the two terms (5.29) and (5.30) are equal. 


\subsubsection{Rayleigh fading links}

For Rayleigh fading links, we have the pdfs for the two links as $f_{\text {nnr }_{1}}(x)=\zeta_{1} e^{-\zeta_{1} x}$ where $\zeta_{1}=1 / \mathrm{SNR}_{1}$ and $f_{\mathrm{snr}_{2}}(x)=\zeta_{2} e^{-\zeta_{2} x}$ where $\zeta_{2}=1 / \mathrm{SNR}_{2}$. Let us first consider the case $\xi \in(0,1)$. From (5.26), we have:

$\mathbb{E}\left\{e^{-\theta\left(1-\phi^{*}[t]\right) r_{1}[t]}\right\}=\int_{0}^{\infty}\left[1-e^{-\zeta_{1} r\left(f\left(x_{2}, \xi\right)\right)}+\zeta_{1}^{\hat{\theta}} e^{\zeta_{1}} \Gamma\left(-\hat{\theta}+1, \zeta_{1}\left(r\left(f\left(x_{2}, \xi\right)\right)+1\right)\right)\right] \zeta_{2} e^{-\zeta_{2} x_{2}} d x_{2}$,

where $\Gamma(s, x)=\int_{x}^{\infty} t^{s-1} e^{-t} d t$ is the incomplete gamma function. Similarly, from (5.27), we have:

$$
\begin{gathered}
\mathbb{E}\left\{e^{-\theta \phi^{*}[t] r_{2}[t]}\right\}=\int_{0}^{r(-\log (1-\xi) / \theta)}\left[1-e^{-\zeta_{2} r\left(f\left(x_{1}, \xi^{-1}\right)\right)}+\zeta_{2}^{\hat{\theta}} e^{\zeta_{2}} \Gamma\left(-\hat{\theta}+1, \zeta_{2}\left(r\left(f\left(x_{1}, \xi^{-1}\right)\right)\right.\right.\right. \\
+1))] \zeta_{1} e^{-\zeta_{1} x_{1}} d x_{1}+\exp \left(-\zeta_{1} r(-\log (1-\xi) / \theta)\right) .
\end{gathered}
$$

We can evaluate these functions using available software such as MATLAB. The expectation terms $(5.29)$ and $(5.30)$ for the case $\xi \in(1, \infty)$ can be computed analogously. We omit the details for brevity.

\subsubsection{Illustration}

For numerical illustration, we fix the average $\mathrm{SNR}_{2}=10 \mathrm{~dB}$ and we plot $\xi$ versus $\theta$ for $\mathrm{SNR}_{1}=5,15 \mathrm{~dB}$ in Figure 5.2. It is observed that $\xi$ increases, and decreases with increasing $\theta$ for $\mathrm{SNR}_{1}<\mathrm{SNR}_{2}$ and $\mathrm{SNR}_{1}>\mathrm{SNR}_{2}$, respectively. Moreover, when $\theta$ becomes larger, $\xi$ approaches 1 in both cases. When $\theta$ becomes sufficiently small, $\xi$ approaches its minimum and maximum values (which is $\xi_{0}$ satisfying (5.22) and (5.24)), respectively.

To further illustrate the effect of delay constraint on the link selection solution, in Figure 5.3 , we plot the following function:

$$
F\left(\theta, r_{2}\right)=-\frac{1}{\theta} \log \left(1+\xi\left(e^{-\theta r_{2}}-1\right)\right)
$$

versus $r_{2}$ for three values of $\theta=10^{-1}, 1$, and 10 for $\mathrm{SNR}_{1}=5 \mathrm{~dB}$ (and hence, $\xi<1$ ). Note that from (5.19), the S-R link is selected in frame $t$ if and only if $r_{1}[t] \geq F\left(\theta, r_{2}[t]\right)$. We 


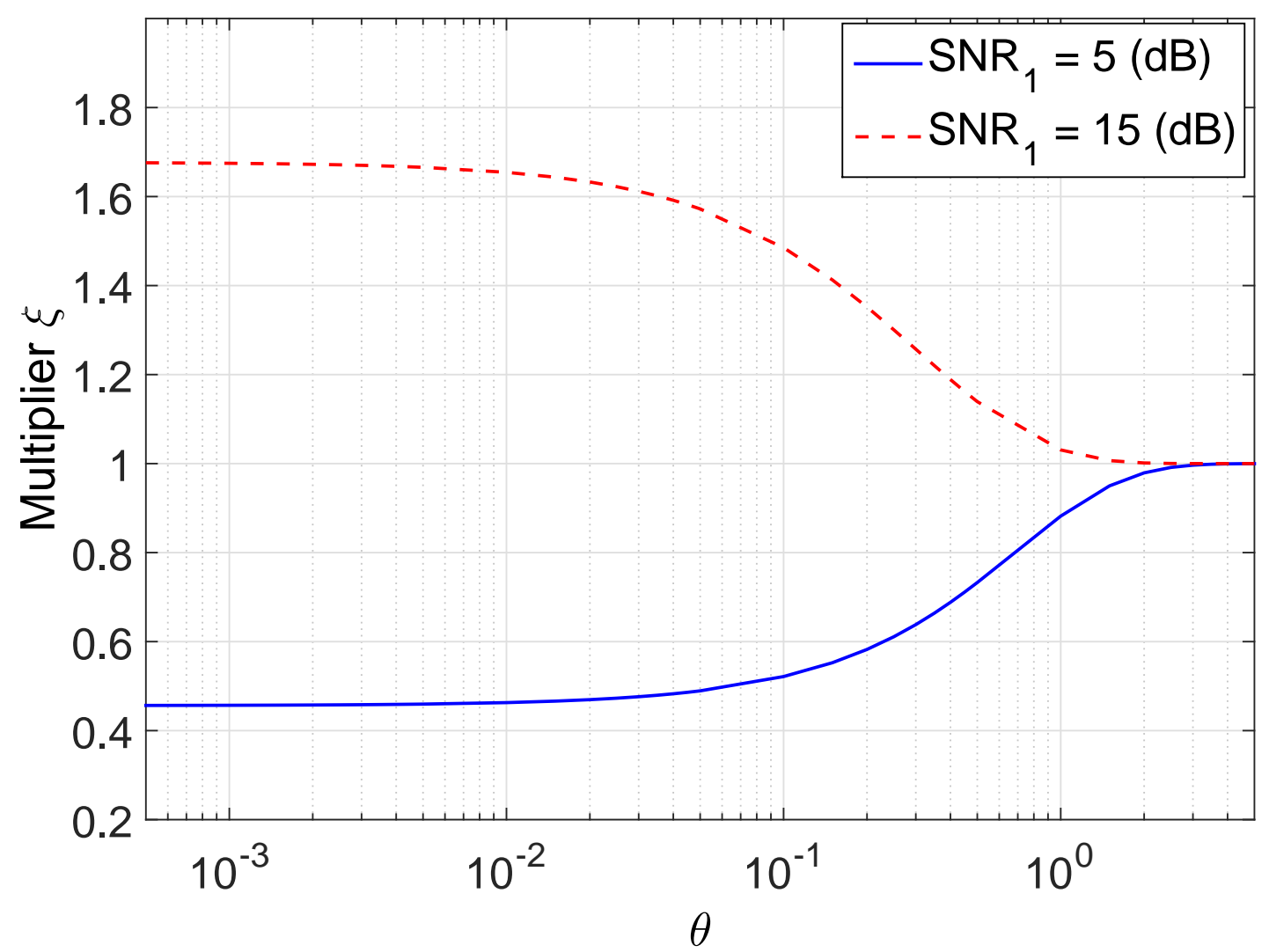

Fig. 5.2: Multiplier $\xi$ versus delay exponent $\theta$ with $\mathrm{SNR}_{2}=10 \mathrm{~dB}$. 
also plot the linear function $\xi_{0} \times r_{2}$. We can observe that when $\theta$ is sufficiently small, e.g., $\theta=10^{-1}, F\left(\theta, r_{2}\right)$ becomes linear as expected. It can be seen that the delay constraint has different impacts on the link selection. For example, for large $\theta$, when $r_{1}[t] \leq 1.8$, the S-R link is selected whenever $r_{1}[t] \geq r_{2}[t]$. However, when $r_{1}[t]>1.8$, the $\mathrm{S}-\mathrm{R}$ link is always selected.

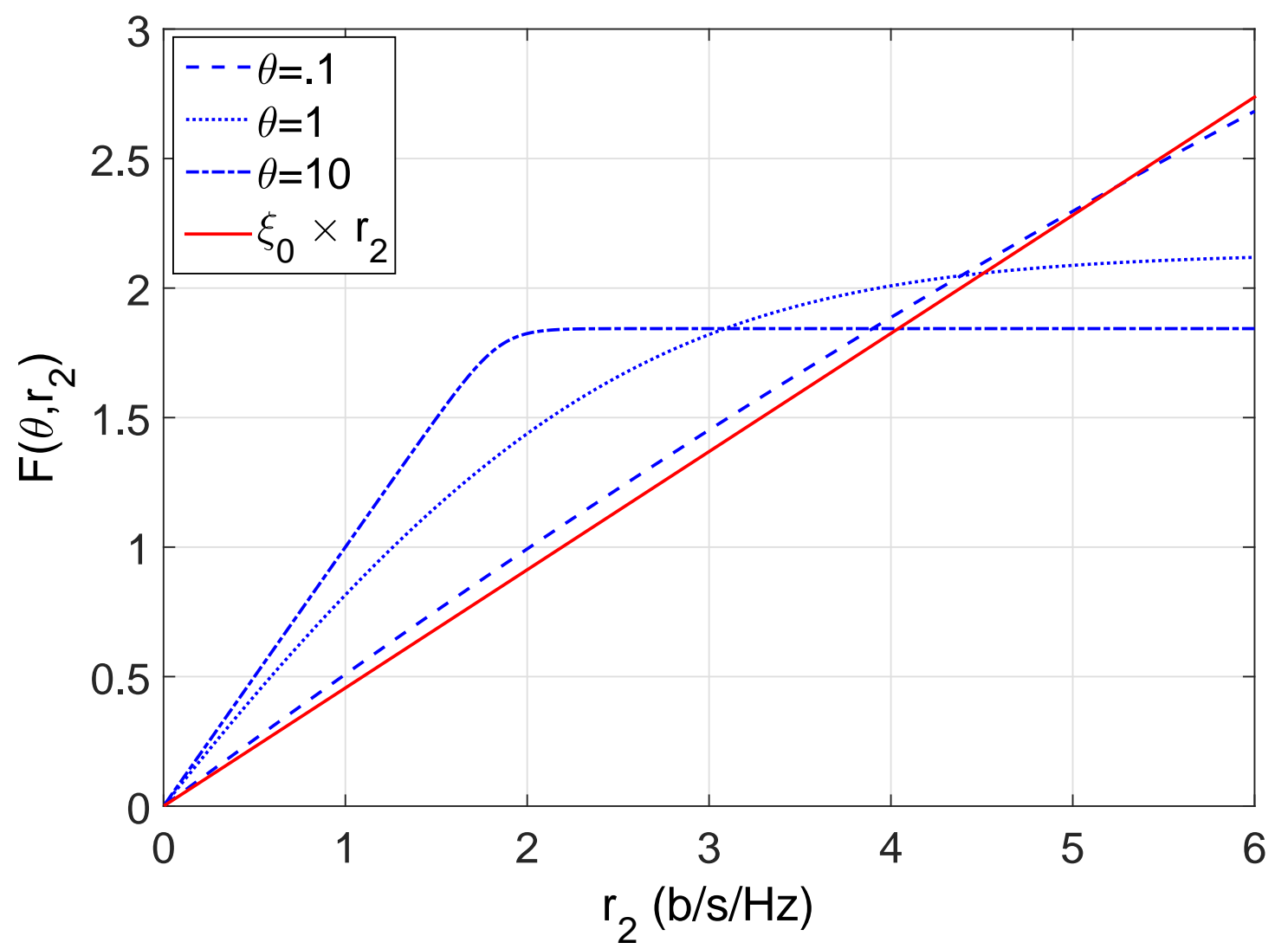

Fig. 5.3: $F\left(\theta, r_{2}\right)$ versus $r_{2}$

\subsubsection{Comparison Benchmarks}

We compare the effective capacity of the proposed QoS-aware B-HD-ALS with those of the QoS-aware B-HD-FLS [79], QoS-blind B-HD-ALS [46], and non-buffer relaying [109]. We next describe these relaying schemes and present how to derive the corresponding effective capacities. 


\subsubsection{QoS-aware B-HD-FLS}

In the fixed relaying, each transmission frame $t$ is divided into two time slots with (possibly) unequal durations. In the first slot of duration $\tau T$ (seconds), $\tau \in(0,1)$, the source transmits packets to the relay with rate $r_{1}[t]$ and in the second slot of duration $(1-\tau) T$ (seconds), the relay transmits its currently buffered packets to the destination with rate $r_{2}[t]$. The effective capacity of the fixed relaying scheme with equal delay exponent $\theta$ at the source and relay is given as [79]:

$$
\mu_{\mathrm{B}-\mathrm{HD}-\mathrm{FLS}}\left(Q^{\max }, \zeta_{Q}\right)=-\frac{1}{\theta} \log \mathbb{E}\left\{e^{-\theta \tau^{\dagger} r_{1}[t]}\right\}
$$

where $\tau^{\dagger} \in(0,1)$ satisfies:

$$
\mathbb{E}\left\{e^{-\theta \tau^{\dagger} r_{1}[t]}\right\}=\mathbb{E}\left\{e^{-\theta\left(1-\tau^{\dagger}\right) r_{2}[t]}\right\}
$$

where $r_{1}[t]$ and $r_{2}[t]$ are given by (5.1).

\subsubsection{QoS-blind B-HD-ALS}

The QoS-blind adaptive relaying scheme maximizes the (ergodic) capacity under unconstrained delay assumption [46]. It has the following form:

$$
\phi[t]= \begin{cases}0, & r_{1}[t] \geq \rho r_{2}[t] \\ 1, & \text { otherwise }\end{cases}
$$

where $\rho$ is determined to maintain the following equality:

$$
\mathbb{E}\left\{(1-\phi[t]) r_{1}[t]\right\}=\mathbb{E}\left\{\phi[t] r_{2}[t]\right\}
$$

We can see that $\rho$ in this case is optimized in consideration of the link fading distributions and average SNR values only. Recall that (5.35) is the limiting case of (5.19) or (5.21) when $\theta$ approaches 0 as we discussed previously. It can be shown that the effective capacity of the QoS-blind relaying scheme is given by:

$$
\mu_{\mathrm{B}-\mathrm{HD}-\mathrm{ALS}}^{\mathrm{QoS}-\mathrm{blind}}\left(Q^{\max }, \zeta_{Q}\right)=\min \left\{-\frac{1}{\theta} \Lambda_{1}(-\theta),-\frac{1}{\theta} \Lambda_{2}(-\theta)\right\}
$$


where $\Lambda_{1}$ and $\Lambda_{2}$ are, respectively, the LMGFs of the service processes of the source and relay queues, which are computed using the relaying scheme (5.34) as follows:

$$
\begin{gathered}
\Lambda_{1}(\theta)=\log \int_{0}^{\infty}\left[\left[\int_{0}^{\left(1+x_{2}\right)^{\rho}-1} 1+\int_{\left(1+x_{2}\right)^{\rho}-1}^{\infty}\left(1+x_{1}\right)^{\theta / \log (2)}\right] f_{\mathrm{snr}_{1}}\left(x_{1}\right) d x_{1}\right] f_{\mathrm{snr}_{2}}\left(x_{2}\right) d x_{2}, \\
\Lambda_{2}(\theta)=\log \int_{0}^{\infty}\left[\left[\int_{0}^{\left(1+x_{1}\right)^{1 / \rho}-1} 1+\int_{\left(1+x_{1}\right)^{1 / \rho}-1}^{\infty}\left(1+x_{2}\right)^{\theta / \log (2)}\right] f_{\mathrm{snr}_{2}}\left(x_{2}\right) d x_{2}\right] f_{\mathrm{snr}_{1}}\left(x_{1}\right) d x_{1} .
\end{gathered}
$$

Note that the first term and the second term in (5.36) are the effective capacities of the S-R link, and R-D link, respectively. The end-to-end effective capacity is the minimum of the two. The proof can be adapted from that in [79] and is omitted for brevity. We can see that the capacity $\mu_{\mathrm{B}-\mathrm{HD}-\mathrm{ALS}}^{\mathrm{QoS}-\mathrm{blind}}=\mu_{\mathrm{B}-\mathrm{HD}-\mathrm{ALS}}$ is achieved only under the following two scenarios:

- either $\theta$ is close to 0 , i.e., very loose delay constraints,

- or the S-R and R-D links have similar fading distribution and average SNR. In this case, the two adaptive relaying schemes are the same where the link with better instantaneous rate is selected.

In other scenarios, it is expected that $\mu_{\mathrm{B}-\mathrm{HD}-\mathrm{ALS}}>\mu_{\mathrm{B}-\mathrm{HD}-\mathrm{ALS}}^{\mathrm{QoS}-\mathrm{blind}}$.

\subsubsection{Non-buffer relaying}

In the non-buffer relaying, (i.e., the relay queue $Q_{2}$ in Fig. 5.1 does not exist), the relay forwards the received packets from the source immediately in the next time-slot. Consider the constant arrival rate $\mu_{\mathrm{N}-\mathrm{HD}}$ to the source buffer and suppose that each frame $t$ is divided into two equal time slots. During the first time-slot, the source transmits packets to the relay. In the second time-slot, the relay decodes and forwards the decoded packets to the destination. As previously defined, $P_{1}$, and $P_{2}$ are the power levels of the source and relay. The achievable rate in frame $t$ is given by:

$$
r[t]=\frac{1}{2} \min \left\{\log _{2}\left(1+P_{1} h_{1}[t]\right), \log _{2}\left(1+P_{2} h_{2}[t]\right)\right\} .
$$


With non-buffer relaying, the packets are delayed at the source buffer only. The effective capacity of the non-buffer relaying is given by [32]:

$$
\mu_{\mathrm{N}-\mathrm{HD}}\left(Q^{\max }, \zeta_{Q}\right)=\frac{-1}{\bar{\theta}_{1}} \log \mathbb{E}\left\{e^{-\bar{\theta}_{1} r[t]}\right\}, \quad \bar{\theta}_{1}=-T B \log \left(\zeta_{Q}\right) / Q^{\max } .
$$

In Section 5.5, we will compare the effective capacities of these relaying schemes under different delay constraints and link average SNR values.

\subsection{Adaptive Link Scheduling with Adaptive Power Allocation}

Previously, we have assumed fixed source and relay transmit power levels $P_{1}$, and $P_{2}$, respectively. However, we can jointly and adaptively optimize the link selection and power allocation in each transmission frame to potentially enhance the capacity as follows. Denote the source and relay transmit power levels in frame $t$ as $P_{1}[t]$ and $P_{2}[t]$, respectively. If $\phi[t]=0$ then $P_{1}[t] \geq 0$ and $P_{2}[t]=0$ while if $\phi[t]=1$ then $P_{1}[t]=0$ and $P_{2}[t] \geq 0$. Then, the average total power is given by $\mathbb{E}\left\{(1-\phi[t]) P_{1}[t]+\phi[t] P_{2}[t]\right\}$.

In this section, we consider the joint adaptive link selection and power allocation problem under the delay-outage and the maximum average power constraints.

\subsubsection{Optimal Solution}

After some simple manipulations, similar to (5.12), the joint adaptive relaying and power allocation problem to maximize the capacity can be equivalently written as:

$$
\begin{array}{cc}
\min _{P_{1}[t] \geq 0, P_{2}[t] \geq 0, \phi[t] \in\{0,1\}} \mathbb{E}\left\{\left(1+P_{1}[t] h_{1}[t]\right)^{-\hat{\theta}(1-\phi[t])}\right\} \\
\text { s.t. : } \quad \mathbb{E}\left\{\left(1+P_{1}[t] h_{1}[t]\right)^{-\hat{\theta}(1-\phi[t])}\right\} \geq \mathbb{E}\left\{\left(1+P_{2}[t] h_{2}[t]\right)^{-\hat{\theta} \phi[t]}\right\}, \\
\mathbb{E}\left\{(1-\phi[t]) P_{1}[t]+\phi[t] P_{2}[t]\right\} \leq \bar{P}^{\max },
\end{array}
$$

where $\bar{P}^{\text {max }}$ denotes the maximum average power constraint.

We can see that at optimality, the inequality constraints in (5.37) must be met with equality. Also, it can be verified that if $P_{1}[t], P_{2}[t]$ are fixed, then the problem (5.37) reduces to the problem (5.12). The effective capacity with joint adaptive link selection and power 
allocation is:

$$
\mu_{\mathrm{B}-\mathrm{HD}-\mathrm{APA}}=\frac{-1}{\hat{\theta} \log (2)} \log \mathbb{E}\left\{\left(1+P_{1}^{*}[t] h_{1}[t]\right)^{-\hat{\theta}\left(1-\phi^{*}[t]\right)}\right\},
$$

where $P_{1}^{*}[t], P_{2}^{*}[t]$, and $\phi^{*}[t]$ are the optimal solutions of (5.37).

To solve (5.37), again, we employ the Lagrangian approach. The Lagrangian of (5.37) is:

$$
\begin{aligned}
\mathcal{L}= & \mathbb{E}\left\{\mathcal{L}[t]=(1-\omega)\left(1+P_{1}[t] h_{1}[t]\right)^{-\hat{\theta}(1-\phi[t])}\right. \\
& \left.+\omega\left(1+P_{2}[t] h_{2}[t]\right)^{-\hat{\theta} \phi[t]}+\sigma\left((1-\phi[t]) P_{1}[t]+\phi[t] P_{2}[t]\right)\right\},
\end{aligned}
$$

where $\omega$, and $\sigma$ are the non-negative Lagrange multipliers associated with the inequality constraints in (5.37). Again, we can see that $\omega \in(0,1)$; otherwise, we would have trivial solution $\phi^{*}[t]=1, \forall t$.

We first study the power allocation solution assuming the link selection solution is given. First, suppose that $\phi^{*}[t]=0$, then we have:

$$
P_{1}^{*}[t]=\underset{P_{1}[t] \geq 0}{\arg \min }\left\{(1-\omega)\left(1+P_{1}[t] h_{1}[t]\right)^{-\hat{\theta}}+\sigma P_{1}[t]\right\} .
$$

The second-order derivative of the objective function of (5.40) is found as:

$$
(1-\omega) \hat{\theta}(\hat{\theta}+1)\left(h_{1}[t]\right)^{2}\left(1+P_{1}[t] h_{1}[t]\right)^{-\hat{\theta}-2}
$$

which is non-negative for $P_{1}[t] \geq 0$. Hence, the objective function is a convex function in $P_{1}[t]$, and (5.40) is a convex optimization problem. Thus, by differentiating the objective function, setting it equal to 0 , and accounting for the non-negativeness of the power allocation, we can derive the optimal power allocation $P_{1}^{*}[t]$ as:

$$
P_{1}^{*}[t]= \begin{cases}\frac{1}{h_{1}[t]}\left(\frac{(1-\omega) \hat{\theta} h_{1}[t]}{\sigma}\right)^{1 /(\hat{\theta}+1)}-\frac{1}{h_{1}[t]}, & h_{1}[t] \geq \frac{\sigma}{(1-\omega) \hat{\theta}} \\ 0, & \text { otherwise. }\end{cases}
$$


Now consider the case $\phi^{*}[t]=1$, then we have:

$$
P_{2}^{*}[t]=\underset{P_{2}[t] \geq 0}{\arg \min }\left\{\omega\left(1+P_{2}[t] h_{2}[t]\right)^{-\hat{\theta}}+\sigma P_{2}[t]\right\} .
$$

Analogous to (5.40), we can derive the optimal power allocation $P_{2}^{*}[t]$ as follows:

$$
P_{2}^{*}[t]= \begin{cases}\frac{1}{h_{2}[t]}\left(\frac{\omega \hat{\theta} h_{2}[t]}{\sigma}\right)^{1 /(\hat{\theta}+1)}-\frac{1}{h_{2}[t]}, & h_{2}[t] \geq \frac{\sigma}{\omega \hat{\theta}} \\ 0, & \text { otherwise }\end{cases}
$$

Using the derived power allocation solutions $P_{1}^{*}[t]$ and $P_{2}^{*}[t]$, we can derive the optimal link selection solution to minimize the Lagrangian (5.39) in each frame as follows:

$$
\phi^{*}[t]=\left\{\begin{array}{cc}
0, & (1-\omega)\left(1+P_{1}^{*}[t] h_{1}[t]\right)^{-\hat{\theta}}+\sigma P_{1}^{*}[t]+\omega \\
& \leq(1-\omega)+\omega\left(1+P_{2}^{*}[t] h_{2}[t]\right)^{-\hat{\theta}}+\sigma P_{2}^{*}[t] \\
1, & \text { otherwise. }
\end{array}\right.
$$

The Lagrange multipliers $\omega$ and $\sigma$ can be determined so that the inequality constraints in (5.37) are met with equalities. We can see that at optimality, all the power budget must be consumed as expected.

To obtain more insights, we study the optimal power allocation policies $P_{1}^{*}[t]$ and $P_{2}^{*}[t]$. By examining $\partial P_{1}^{*}[t] / \partial h_{1}[t]$ (or $\partial P_{2}^{*}[t] / \partial h_{2}[t]$ ), we can see that $P_{1}^{*}[t]$ (or $P_{2}^{*}[t]$ ) increases with increasing $h_{1}[t]$ when $h_{1}[t]$ is less than a threshold value and then decreases with increasing $h_{1}[t]$ otherwise. Hence, the power allocation solutions swing between the water-filling (for smaller $h_{1}[t]$ ) and channel inversion (for larger $h_{1}[t]$ ) policies.

The maximum sum power constraint has been considered in many scenarios [114]- [117]. Although the source and relay may not be able to share the power, the maximum sum power constraint allows performance comparison with other schemes to support the same source-destination path (e.g., direct source-destination link, buffer-aided relaying, non-buffer relaying, QoS-aware relaying, QoS-blind relaying, fixed relaying, adaptive relaying, and so on) in a fair manner on the basis of the same maximum total power constraint. Furthermore, the total power constraint is a way to limit the interference from one network to other coexisting networks. 


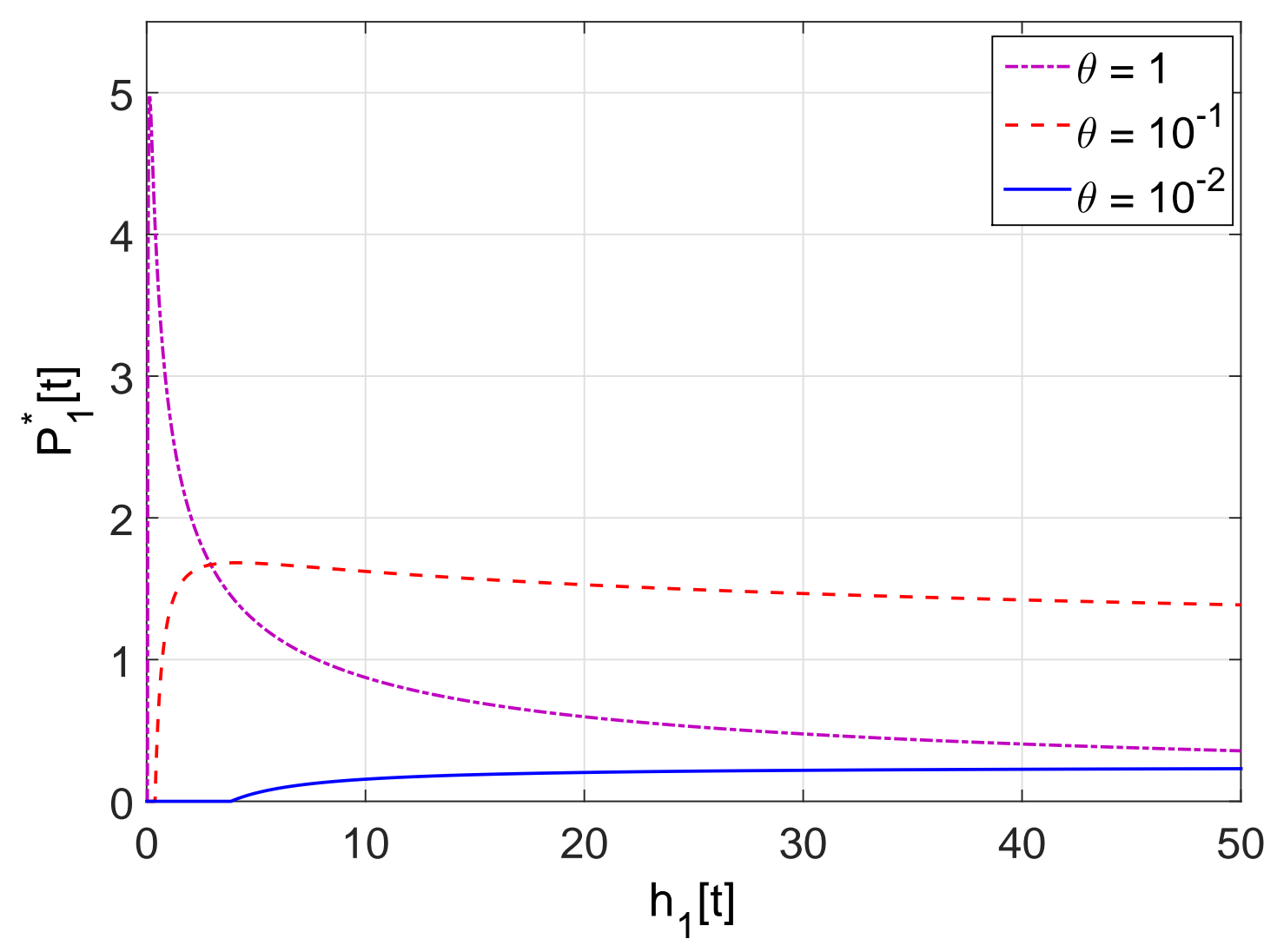

Fig. 5.4: Optimal power allocation $P_{1}^{*}[t]$ versus $h_{1}[t]$. 
The proposed solution approach can be extended to study the problem with individual power constraints at the source and relay. By introducing three Lagrange multipliers: one for the delay constraint and the other two for the source and relay individual power constraints, the link selection and power allocation solution in each frame $t$ depends on these Lagrange multipliers. It can be verified that the delay constraint and at least one of the two power constraints must hold with equality at optimality. Detailed analysis of each possible case must be developed to determine the Lagrange multipliers.

\subsubsection{Special Cases}

\subsubsection{Case of very loose delay constraints}

When the delay constraint becomes very loose, i.e., $\hat{\theta}$ is small, the power allocation $P_{1}^{*}[t]$ in (5.41) and $P_{2}^{*}[t]$ in (5.43) approach the following:

$$
P_{1}^{*}[t]= \begin{cases}\frac{(1-\omega) \hat{\theta}}{\sigma}-\left(h_{1}[t]\right)^{-1}, & h_{1}[t] \geq \sigma /((1-\omega) \hat{\theta}), \\ 0, & \text { otherwise }\end{cases}
$$

and

$$
P_{2}^{*}[t]= \begin{cases}\frac{\omega \hat{\theta}}{\sigma}-\left(h_{2}[t]\right)^{-1}, & h_{2}[t] \geq \sigma /(\omega \hat{\theta}), \\ 0, & \text { otherwise. }\end{cases}
$$

Hence, the power allocation solutions tend to the conventional water-filling policies, albeit with two different water-levels. Again, this result has been derived in [46] under infinite delay assumption.

\subsubsection{Case of very stringent delay constraints}

When the delay constraint becomes more stringent, i.e., $\hat{\theta}$ becomes large, the power allocation $P_{1}^{*}[t]$ in (5.41) and $P_{2}^{*}[t]$ in (5.43) become:

$$
P_{1}^{*}[t]=\frac{1}{h_{1}[t]}\left[\left(\frac{(1-\omega) \hat{\theta}}{\sigma}\right)^{1 /(\hat{\theta}+1)}-1\right], \quad h_{1}[t] \geq \frac{\sigma}{(1-\omega) \hat{\theta}}
$$




\section{Resource Allocation for Buffer-Aided Half-Duplex Relaying under Delay-Outage Constraint}

and

$$
P_{2}^{*}[t]=\frac{1}{h_{2}[t]}\left[\left(\frac{\omega \hat{\theta}}{\sigma}\right)^{1 /(\hat{\theta}+1)}-1\right], \quad h_{2}[t] \geq \sigma /(\omega \hat{\theta})
$$

which are similar to the channel-inversion policies. We re-emphasize that under very stringent delay constraints, the effective capacity still approaches 0 , even under optimal power allocation.

Intuitively, under loose delay constraints, since the queue length bound violation is allowed with large probability, more data is stored in the (source and relay) queues for longer duration. Hence, it is better to transmit at higher power under more favorable channel conditions to exploit the temporal fading diversity, i.e., the water-filling policy. On the other hand, under more stringent delay constraints, in order to avoid queue length bound violation, it is better to transmit at lower power levels under more favorable channel conditions, i.e., channel-inversion policy. For illustration, in Fig. 5.4, we plot $P_{1}^{*}[t]$ versus $h_{1}[t]$ for $\omega=0.1$, $\sigma=0.05$, and different values of $\theta=10^{-2}, 10^{-1}$, and 1 . This figure shows that the power allocation policies approach the water-filling and channel-inversion policies for small and large $\theta$, respectively, as analyzed. For medium $\theta$, the allocation policy swings between these two policies as we discussed above.

\subsubsection{Resource Allocation Algorithm over Unknown Fading Links}

The link selection and power allocation solutions depend on the fading statistics through the Lagrange multipliers $\omega$ and $\sigma$, which are determined so that the inequality constraints (5.37) are met with equality. We can use a (two-dimension) numerical search method as in the case of fixed power allocation. However, such numerical approach has two possible limitations: 1) It requires the fading statistics to be known, which is usually not the case in reality; 2) Even when the fading statistics are known, it may be complicated, if not impossible, to compute the expectation terms in closed-form which are then used for numerical computation. Hence, in general, it may not be possible to employ the numerical method to compute the Lagrange multipliers $\omega$ and $\sigma$. To overcome these limitations, we can utilize the following online allocation algorithm. We initialize the Lagrange multipliers with $\omega[1] \in(0,1)$, and $\sigma[1]>0$. 
Then, in transmission frame $t=1,2, \ldots$, we carry out the following updates:

$$
\begin{aligned}
& \omega[t+1]=\left[\omega[t]+\epsilon[t]\left(\left(1+P_{2}^{*}[t] h_{2}[t]\right)^{-\hat{\theta} \phi^{*}[t]}-\left(1+P_{1}^{*}[t] h_{1}[t]\right)^{-\hat{\theta}\left(1-\phi^{*}[t]\right)}\right)\right]_{\varepsilon}^{1} \\
& \sigma[t+1]=\left[\sigma[t]+\epsilon[t]\left(\left(1-\phi^{*}[t]\right) P_{1}^{*}[t]+\phi^{*}[t] P_{2}^{*}[t]-\bar{P}^{\max }\right)\right]_{\varepsilon}^{L}
\end{aligned}
$$

for small positive coefficient $\varepsilon$, where $[x]_{a}^{b}$ denotes the projection of $x$ on the interval $[a, b]$ for $a \leq b$ and $L$ is sufficiently large to ensure boundedness of $\sigma[t+1]$. The decreasing positive sequence $\epsilon[t]$ that dictates the convergence speed, satisfies:

$$
\sum_{t=1}^{\infty} \epsilon[t]=\infty ; \quad \sum_{t=1}^{\infty}(\epsilon[t])^{2}<\infty
$$

The allocation solutions $P_{1}^{*}[t], P_{2}^{*}[t]$, and $\phi^{*}[t]$ in frame $t$ are computed using the current estimates $\omega[t]$ and $\sigma[t]$. These iterative stochastic-approximation updates are guaranteed to converge to the optimal multipliers. We can see that these updates do not require the fading statistical knowledge and have very low implementation complexity. Moreover, the allocation algorithm does not assume any specification on the fading statistics, and it converges for any independent link fading distributions. Hence, it is very robust to channel model variations.

\subsection{Illustrative Results}

\subsubsection{Settings}

For illustrative purposes, we assume Rayleigh fading links with block-fading duration $T=5$ ms, the bandwidth $B=10 \mathrm{kHz}$. For the delay-outage constraint, we fix the queue length bound $Q^{\max }=3000$ (bits) to obtain numerical results in this section. The queue-lengthoutage probability $\zeta_{Q}$ can be fixed or varied depending on the simulation scenarios.

\subsubsection{Fixed Power Allocation}

For a given delay constraint (5.2), we wish to demonstrate that by provisioning similar delay statistics (performance)at the source and relay, i.e., $\theta_{1}=\theta_{2}$, we can achieve the largest effective capacity. 


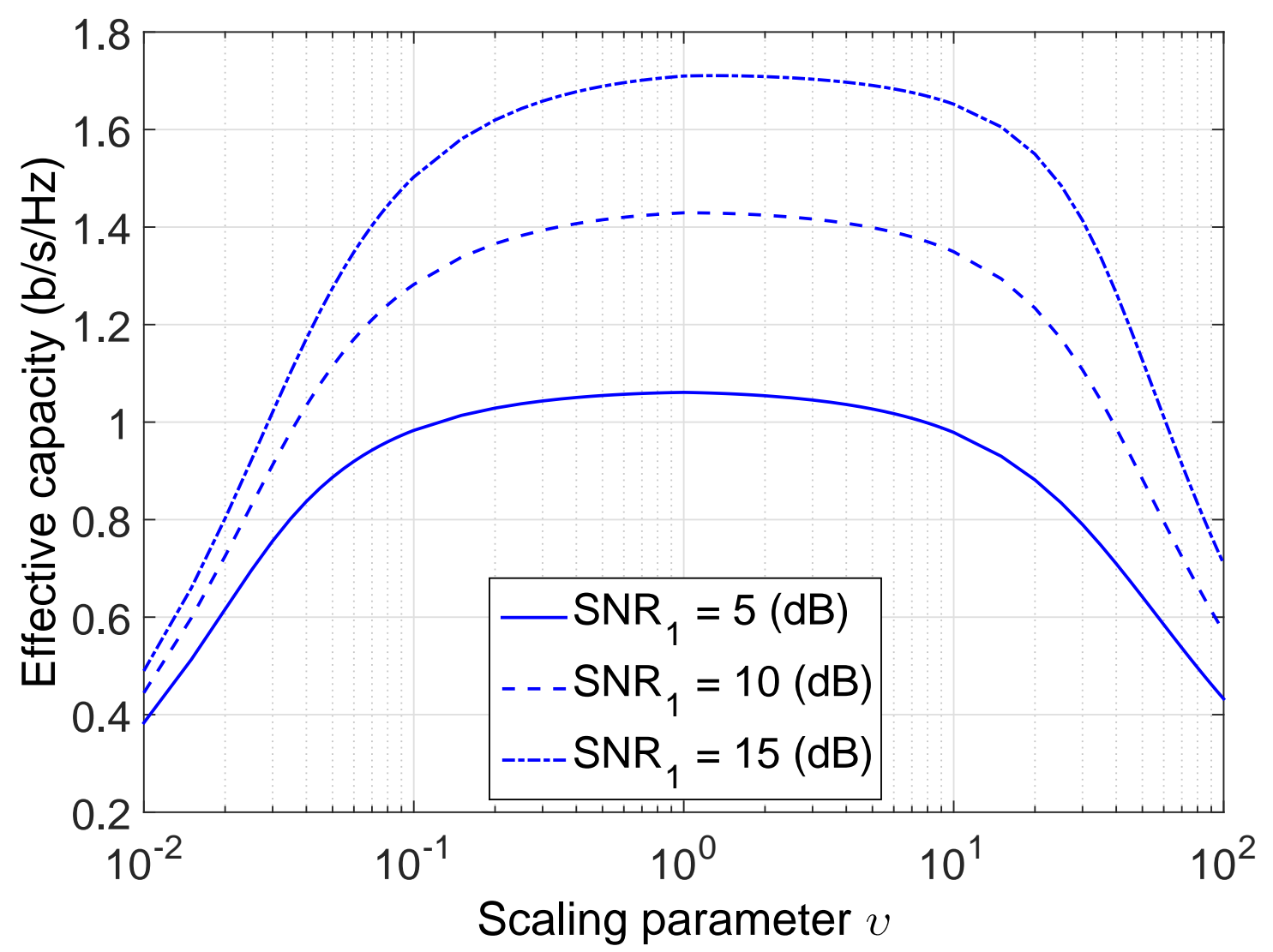

Fig. 5.5: Effective capacity versus scaling parameter $v$.

We fix $\zeta_{Q}=10^{-2}$ and $\mathrm{SNR}_{2}=10 \mathrm{~dB}$ and assume $\theta_{1}=v \theta_{2}$ where the scaling parameter $v>0$ represents the relative required delay performance at the source and relay queues. In particular, $v<1$ means that more delay is allowed at the source than the relay due to $\theta_{1}<\theta_{2}$. On the other hand, $v>1$ means that more delay is allowed at the relay. In Figure 5.5 , we plot the capacity versus $v \in\left[10^{-2}, 10^{2}\right]$ for $\mathrm{SNR}_{1}=5,10,15 \mathrm{~dB}$. We use (5.4) to compute the delay exponents $\theta_{1}$ and $\theta_{2}$ corresponding to each value of $v$. We can see that for all three values of $\mathrm{SNR}_{1}$, the capacities are largest when $v=1$, i.e., $\theta_{1}=\theta_{2}$. Even when the links have different signal strengths, applying the same delay exponent at the source and relay queues achieves the largest capacity. This is because the QoS-aware relaying scheme takes into account the delay allocation at the source and relay and link average SNR values. In contrast, it can be observed that provisioning different delay performance at the source 
and relay reduces the capacity. We study the power allocation at source and relay to achieve

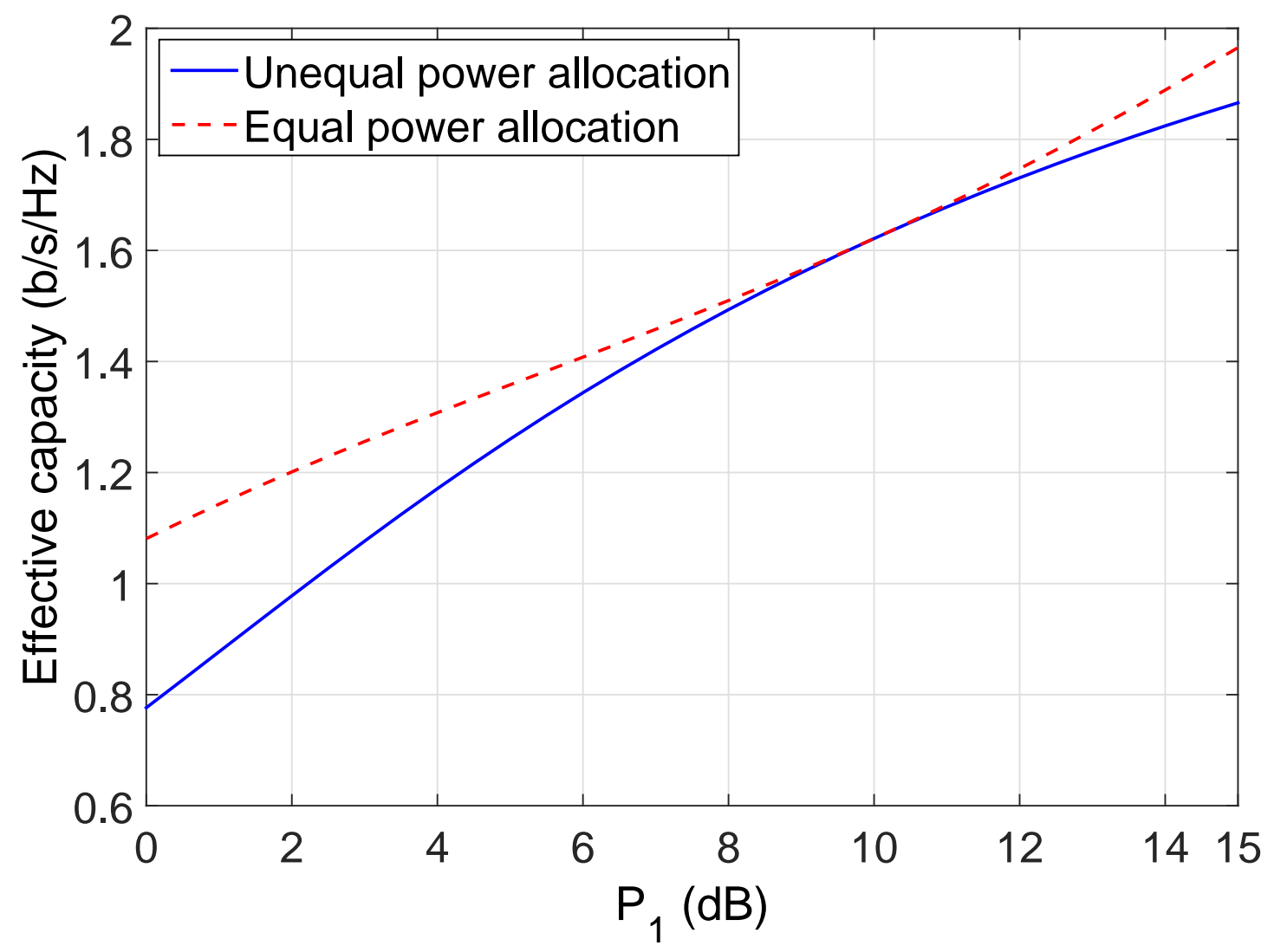

Fig. 5.6: Effective capacity versus $P_{1}$.

the largest capacity under the same average total power constraint. Note that this power allocation is determined before transmission (i.e., off-line calculation). For this purpose, we fix $\zeta_{Q}=10^{-2}$ and set up the experiment as follows.

First, we assume that $\mathbb{E}\left\{h_{1}\right\}=\mathbb{E}\left\{h_{2}\right\}=1$ for convenience. Hence, $P_{1}$ and $P_{2}$ are also the average link SNR values. Now, for unequal power allocation at the source and relay, we fix $P_{2}=10 \mathrm{~dB}$ and vary $P_{1}$. For each value of $P_{1}$, we determine the optimal B-HD-ALS relaying scheme and compute its corresponding capacity $\mu^{\mathrm{UNEQ}}$. We also compute the average total power as follows:

$$
\bar{P}=\operatorname{Pr}\left(\phi^{*}[t]=0\right) P_{1}+\operatorname{Pr}\left(\phi^{*}[t]=1\right) P_{2} .
$$

We next compute the capacity $\mu^{\mathrm{EQ}}$ of the B-HD-ALS relaying scheme with equal source 
and relay transmit power. To ensure that the two schemes utilize the same average power, in the latter scheme, the source or the relay transmit with power $P_{1}=P_{2}=\bar{P}$ in (5.44) if active in each frame. In Figure 5.6, we plot the capacities versus $P_{1}$. We can see that equal power allocation at the source and relay, and hence, equal link average SNR, achieves the highest capacity. Otherwise, the link with smaller average SNR becomes the bottleneck link that reduces the capacity even though B-HD-ALS does consider the link average SNR values. However, note that equal average SNR might not achieve the highest capacity if the links have different fading distributions.

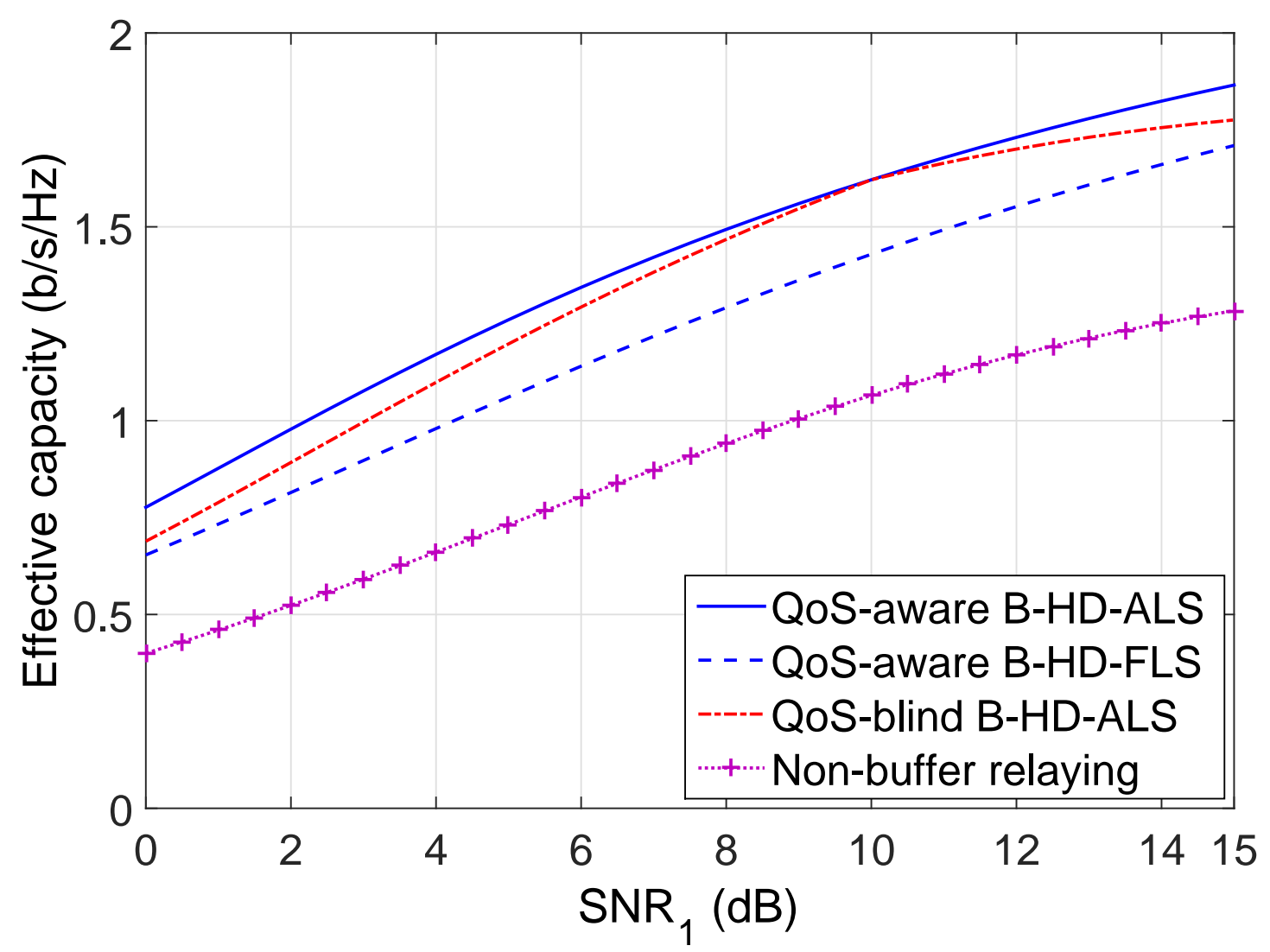

Fig. 5.7: Effective capacity versus $\mathrm{SNR}_{1}$.

We numerically compare the capacities of the proposed QoS-aware B-HD-ALS and the other relaying schemes: 1) QoS-aware B-HD-FLS [79]; 2) QoS-blind B-HD-ALS [46]; 3) Nonbuffer relaying. The capacities of these schemes are described in Section 5.3.3. To conduct 
the comparisons, we fix $\mathrm{SNR}_{2}=10 \mathrm{~dB}$.

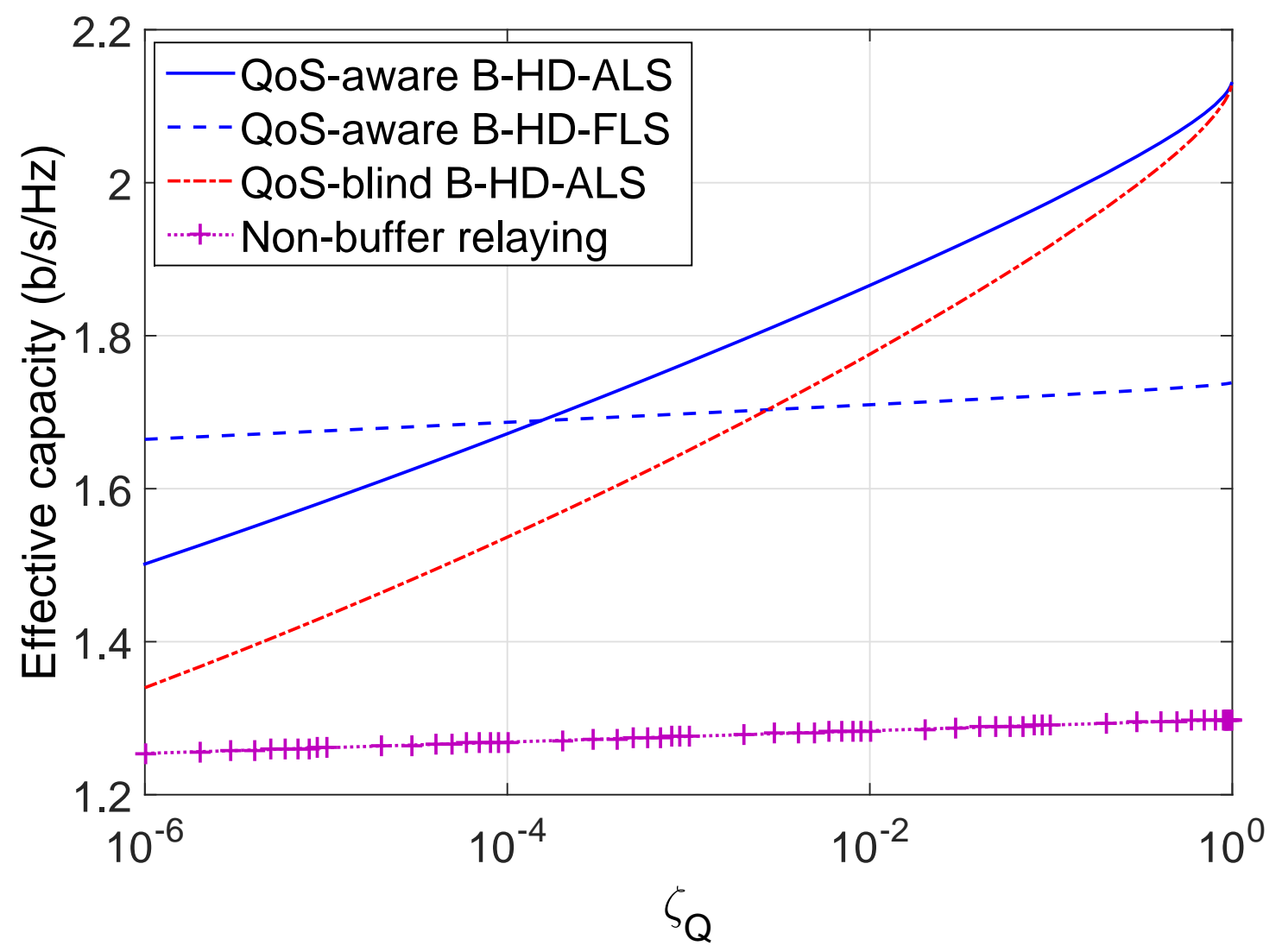

Fig. 5.8: Effective capacity versus $\zeta_{Q}$.

First, we set $\zeta_{Q}=10^{-2}$. Figure 5.7 shows the capacities of the relaying schemes versus $\mathrm{SNR}_{1}$. It can be observed that the QoS-aware B-HD-ALS attains the highest capacity for all values of $\mathrm{SNR}_{1}$. Note that when $\mathrm{SNR}_{1}=10 \mathrm{~dB}$, the QoS-aware and QoS-blind B-HD-ALS schemes are similar, hence, they achieve similar capacity. Also, QoS-blind B-HD-ALS does not capitalize on the increased $\mathrm{SNR}_{1}$ when $\mathrm{SNR}_{1}>\mathrm{SNR}_{2}$ because the $\mathrm{S}-\mathrm{R}$ link is forced to transmit less often resulting in small supportable arrival rates. Moreover, it can be seen that the gain due to adaptive link selection relaying over fixed relaying is reduced when $\mathrm{SNR}_{1}$ becomes larger. In this case, fixed relaying performs well since both links now have favorable channel conditions in each frame. When $\mathrm{SNR}_{1}$ is small, the $\mathrm{S}-\mathrm{R}$ link is still active in each frame even if it might have unfavorable channel conditions, which leads to reduced 
capacity for the fixed relaying. We can see that buffer-aided relaying is more effective than non-buffer relaying to support delay-sensitive applications because in non-buffer relaying, the end-to-end rate is dominated by the weaker of the two links.

Figure 5.8 shows the capacities of the relaying schemes versus $\zeta_{Q}$ for $\mathrm{SNR}_{1}=15 \mathrm{~dB}$. It is clear that as $\zeta_{Q}$ becomes larger, higher capacities can be achieved for all schemes. For each case, we can see that adaptive relaying outperforms fixed relaying when $\zeta_{Q}$ is higher than a certain threshold. QoS-aware relaying performs better than QoS-blind relaying for all queue-length-outage probabilities as shown previously and the gain becomes smaller under looser delay constraints.

\subsubsection{Adaptive Power Allocation}

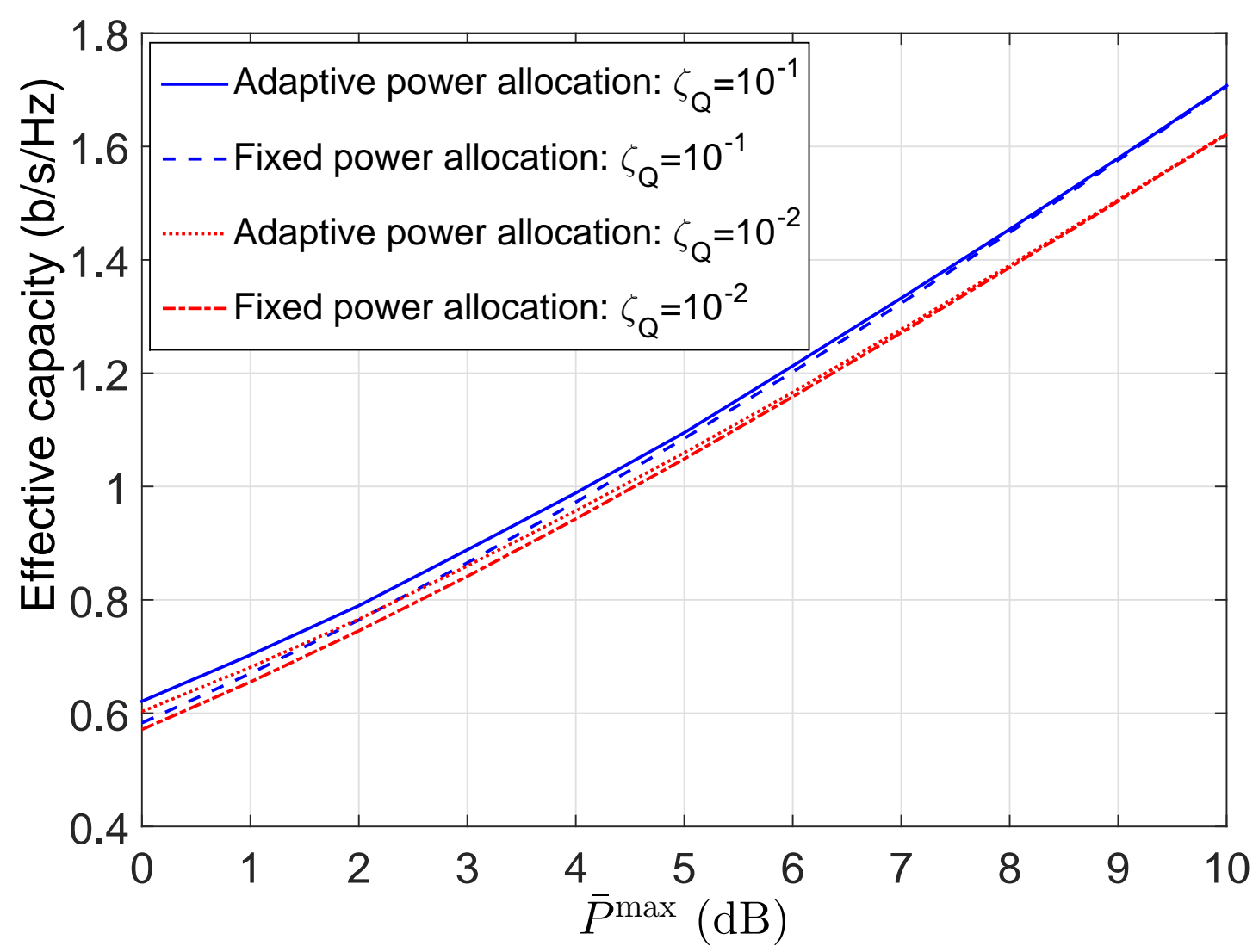

Fig. 5.9: Effective capacity versus $\bar{P}^{\max }$. 


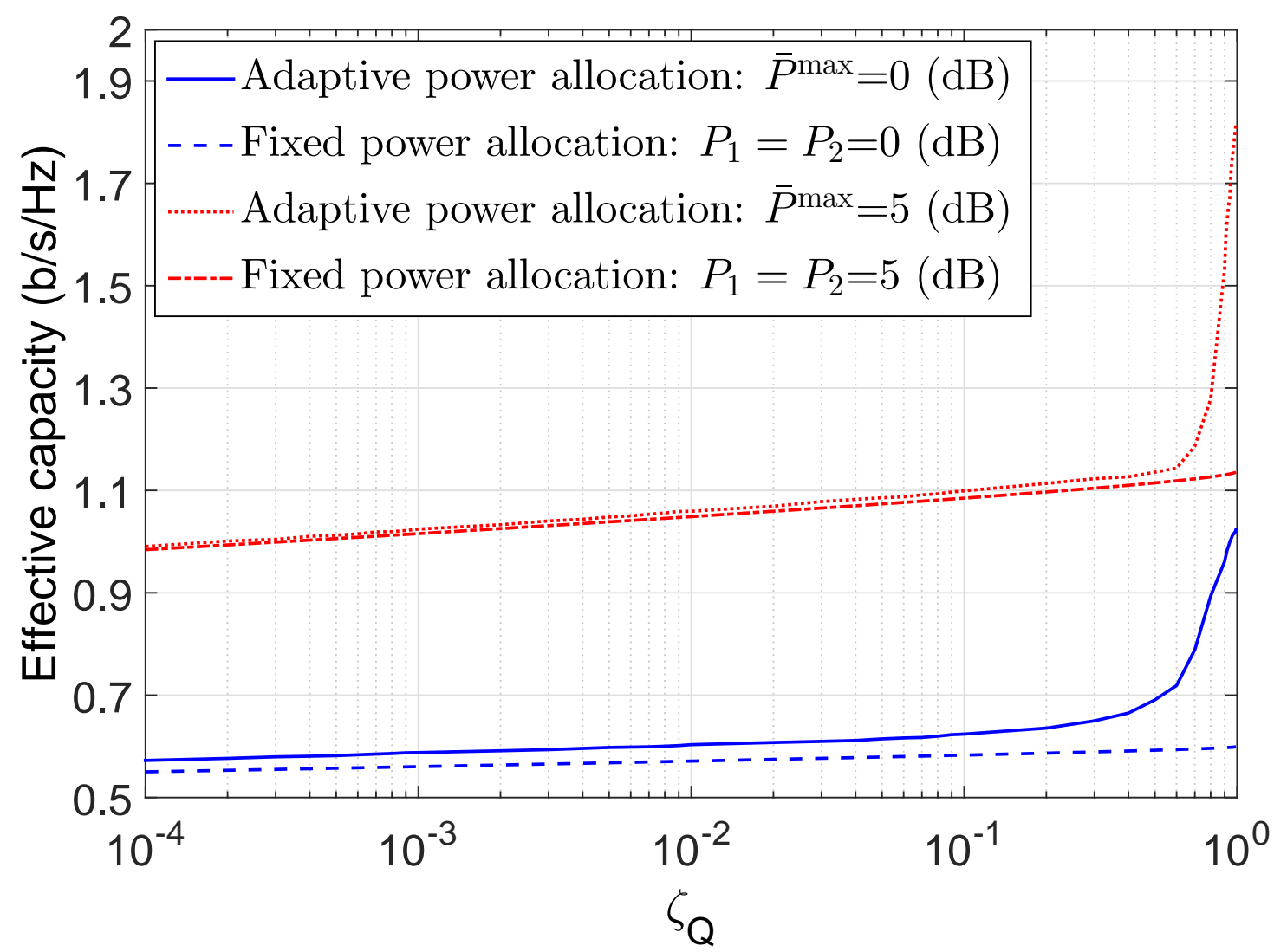

Fig. 5.10: Effective capacity versus $\zeta_{Q}$.

In this numerical study, we assume that $\mathbb{E}\left\{h_{1}\right\}=\mathbb{E}\left\{h_{2}\right\}=1$ for convenience.

We study the capacity gains achieved by the adaptive power allocation over fixed power allocation. We assume equal average power consumption $\bar{P}^{\text {max }}$ in both relaying schemes. For fixed power allocation, we further assume $P_{1}=P_{2}=\bar{P}^{\text {max }}$ in order to attain the highest capacity as studied in Figure 5.6. For the case that $P_{1} \neq P_{2}$ in fixed power allocation scheme, we expect to achieve higher gains due to power adaption for the same average power.

Figure 5.9 shows the capacities of the relaying schemes versus $\bar{P}^{\text {max }}$ for different $\zeta_{Q}=$ $10^{-2}, 10^{-1}$. It can be observed that power adaptation is more beneficial at lower SNR, e.g., $\bar{P}^{\max } \leq 5 \mathrm{~dB}$. This can be explained as follows. At low SNR, since the capacity is dominated by the transmission rates, and hence, by adaptively allocating the power to the source and relay to exploit the link and temporal diversities, higher capacity gains can be achieved. 


\section{Resource Allocation for Buffer-Aided Half-Duplex Relaying under Delay-Outage Constraint}

On the other hand, at high SNR, the capacity is dominated by the delay constraint, and hence, power adaption is not much advantageous. As a result, under more stringent delay constraints, the gains due to power adaptation are expected to be reduced. This observation is further confirmed in the following investigation.

We plot the capacities of the relaying schemes versus $\zeta_{Q}$ for average power $\bar{P}^{\max }=0,5$ $\mathrm{dB}$ in Figure 5.10. We can see that power adaptation is less beneficial under more stringent delay constraints because the capacity is dominated by the delay constraints. Particularly, for large $\bar{P}^{\text {max }}$, the gains are marginal under stringent delay constraints. However, under looser delay constraints, power adaption can provide significant capacity gains over fixed power allocation. Since data transmissions can be delayed for longer time, temporal diversity can be exploited better by transmitting more under more favorable channel conditions and vice versa. From the above numerical studies, we can conclude that power adaption is useful when sufficiently long delay can be tolerated and/or when the power budget is limited.

\subsection{Chapter Summary}

In this chapter, we have studied buffer-aided half-duplex (B-HD) relaying network over fading channels under the delay-outage constraint. To exploit the buffer-aided relaying capability and link fading diversity, B-HD with adaptive link selection (B-HD-ALS) relaying schemes with fixed and adaptive source and relay power allocation are proposed. In each transmission frame, the relay can be activated adaptively to receive packets from the source or to transmit packets to the destination depending on the instantaneous channel state information. The link selection and power allocation solutions are derived to maximize the constant supportable arrival rate $\mu$ to the source using Lagrangian approach and convex optimization. The effects of the delay constraint on the derived solutions are identified. Illustrative results compare the capacities of the proposed relaying schemes and other existing schemes. In general, the simulation results show that B-HD-ALS is beneficial as long as a certain delay can be tolerated. 


\section{Chapter 6}

\section{Power Allocation for Buffer-Aided Full-Duplex Relaying with Imperfect Self-Interference Cancellation under Delay-Outage Constraint}

In Chapter 5, we have considered half-duplex (HD) relaying where the relay either transmits or receives in each transmission frame to avoid self-interference (SI) at the expense of low spectral efficiency. Recently-developed SI mitigation methods can leverage the potential full-duplex (FD) relaying, in which a relay can receive and transmit simultaneously over the same frequency. However, SI cannot be completely mitigated in practice. In this chapter, we consider a buffer-aided FD (B-FD) relaying with imperfect SI cancellation, where the nonzero residual SI is assumed to be proportional with parameter $\beta>0$ to the relay transmit power. We investigate two source and relay transmit power allocation problems for effective capacity maximization, which depend on the availability of the channel state information at the transmitters (CSIT).

First, when the instantaneous CSIT is available, we investigate the B-FD relaying with adaptive power allocation (B-FD-APA) problem. The source and relay powers are adaptively allocated in each frame depending on the channel conditions. Depending on the

Parts of Chapter 6 are presented at the IEEE International Symposium on Personal, Indoor and Mobile Radio Communications (PIMRC) in Hong Kong, China [118], and published in the IEEE Access [119]. 
channel conditions, the relay can switch between HD and FD operation modes, i.e., hybrid $\mathrm{HD} / \mathrm{FD}$ relaying [52]. Moreover, the optimal B-FD-APA solutions for special cases on the delay constraint and SI cancellation parameter $\beta$ are also studied. In particular, for large $\beta$ and loose delay constraint, B-FD-APA approaches buffer-aided HD (B-HD) relaying with adaptive link scheduling (B-HD-ALS) [108].

Second, it is known that instantaneous CSIT is not always possible in wireless systems due to CSI feedback complexity from the receivers. Then, the source and relay might not be able to adapt their transmit powers efficiently and need to transmit with fixed powers. On the other hand, statistical CSIT (or link fading statistics) is more accessible than instantaneous CSIT since the duration over which the fading processes are stationary is much longer than the duration of the fades. By exploiting the statistical CSIT, we study the B-FD relaying with static power allocation (B-FD-SPA) problem. The optimal B-FD-SPA solution is derived taking into account the fading statistics, SI cancellation parameter $\beta$, as well as the delay constraint. Several properties of the optimal B-FD-SPA solution are investigated. Also, solutions for various special cases on the delay constraint and $\beta$ are analyzed.

Furthermore, to investigate the potential benefits of B-FD relaying over non-buffer FD (N-FD) relaying, we also study two power allocation problems in N-FD relaying under delayoutage constraint: 1) N-FD relaying with adaptive power allocation (N-FD-APA) when instantaneous CSIT is available; 2) N-FD relaying with static power allocation (N-FD-SPA) when only statistical CSIT is available. The solutions for both problems are derived. Illustrative results are performed to demonstrate the effectiveness of the proposed relaying schemes over the existing relaying schemes (e.g., B-HD relaying and N-FD relaying).

The rest of the chapter is organized as follows. In Section 6.1, the system model is presented, and the power allocation problems are formulated. The solution approaches for the B-FD-APA and B-FD-SPA problems are presented in Sections 6.2, and 6.3, respectively. In Section 6.4, N-FD relaying problems are described. Numerical studies are presented in Section 6.5 and Section 6.6 presents the conclusion. 


\subsection{System Model and Problem Formulations}

\subsubsection{Model Description}

Consider a decode-and-forward buffer-aided relaying network where a source (S) communicates with its destination (D) with the help of an intermediate relay (R) with FD relaying capability over a channel with bandwidth $B(\mathrm{~Hz})$ as shown in Fig. 6.1. Data packets are assumed to arrive at the source buffer with constant rate $\mu$.

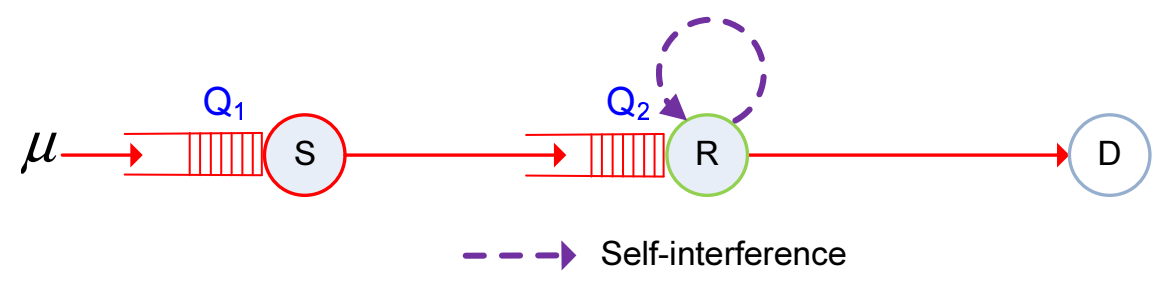

Fig. 6.1: B-FD relaying model.

We consider block-fading channels with fading duration $T$ (seconds) which is equal to the transmission frame duration, i.e., the instantaneous channel power gains in frame $t=1,2, \ldots$, $h_{i}[t], i=1,2$ of the S-R and R-D links, respectively, remain unchanged during frame $t$ but vary independently from frame to frame. Moreover, $h_{i}[t], i=1,2$ are assumed to be statistically independent with means $\mathbb{E}\left\{h_{i}[t]\right\}$. We assume that perfect instantaneous channel state information (CSI) is available at the receivers in each frame, i.e., the relay knows $h_{1}[t]$ and the destination knows $h_{2}[t]$.

Let $P_{1}[t], P_{2}[t] \geq 0$ denote the source and the relay transmit power levels in frame $t$, respectively. It can be seen that, in HD relaying, at most one of $P_{1}[t]$ or $P_{2}[t]$ is positive, i.e., the relay cannot simultaneously receive and transmit, while in FD relaying, it is possible that both $P_{1}[t]$ and $P_{2}[t]$ are non-zero. Moreover, in FD relaying, the residual SI is assumed to be zero-mean, additive and Gaussian with the variance proportional to the relay transmit power $P_{2}[t]$ as commonly assumed in existing literature [51]- [53], i.e., $\beta P_{2}[t]$ represents the residual SI power, and the parameter $\beta \geq 0$ represents the SI cancellation quality. Larger $\beta$ implies worse SI cancellation performance. In this work, we study two following power allocation schemes for $P_{1}[t]$, and $P_{2}[t]$.

Case 1: Adaptive power allocation (B-FD-APA). When instantaneous CSIT is available, $P_{1}[t]$, and $P_{2}[t]$ are adaptively allocated in each frame $t$ according to $h_{1}[t]$, and $h_{2}[t]$. The 
instantaneous signal-to-SI-plus-noise power ratio (SINR) of the S-R link and signal-to-noise power ratio (SNR) of the R-D link can be expressed as:

$$
\operatorname{sinr}_{1}[t]=\frac{h_{1}[t] P_{1}[t]}{\beta P_{2}[t]+1}, \quad \operatorname{snr}_{2}[t]=h_{2}[t] P_{2}[t]
$$

where the noise power is taken as reference and normalized to 1 . It is noted that the path loss (including both transmitter and receiver antenna gains) is also normalized to $1 . \beta P_{2}[t]$ also represents the residual-SI-to-noise ratio (INR). The instantaneous transmission rates of the S-R link and R-D link (b/s/Hz) are:

$$
r_{1}[t]=\log _{2}\left(1+\operatorname{sinr}_{1}[t]\right), \quad r_{2}[t]=\log _{2}\left(1+\operatorname{snr}_{2}[t]\right) .
$$

Note that instantaneous CSIT is achieved by using feedback from the receiver. Since there is feedback delay, instantaneous CSIT is meaningful when the fading block duration is (much) larger than the CSI feedback delay.

Case 2: Static power allocation (B-FD-SPA). When only statistical CSIT is available, the source and relay might not be able to adapt their transmit powers efficiently. In this case, they should transmit with constant powers over frames, i.e., $P_{1}[t]=P_{1}>0$, and $P_{2}[t]=P_{2}>0, \forall t$. The instantaneous transmission rates of the S-R link and R-D link are:

$$
r_{1}[t]=\log _{2}\left(1+\frac{h_{1}[t] P_{1}}{\beta P_{2}+1}\right), \quad r_{2}[t]=\log _{2}\left(1+h_{2}[t] P_{2}\right)
$$

In (6.3), it is assumed that the transmission rates are still given by (6.2) and hence the transmitters employ variable-rate transmission schemes, even though the transmitters do not know the instantaneous CSI [65], [120]. This can be accomplished by using recently developed rateless codes such as LT codes [121], Raptor codes [122], which enable the transmitter to adapt its rate to the channel realization without requiring CSIT [123]. We omit the details here.

As shown in Fig. 6.1, using the above notations, the service processes of the source and the relay queues are $\left\{T B r_{1}[t]\right\}$, and $\left\{T B r_{2}[t]\right\}$, respectively where $r_{1}[t]$ and $r_{2}[t]$ are given by (6.2) (for B-FD-APA) or (6.3) (for B-FD-SPA). Let $q_{1}[t], q_{2}[t] \geq 0$ denote the source and relay queue lengths in frame $t$, respectively. Then, the corresponding queue length dynamics 
are given as:

$$
\begin{aligned}
& q_{1}[t+1]=q_{1}[t]-\min \left\{q_{1}[t], T B r_{1}[t]\right\}+\mu T B \\
& q_{2}[t+1]=q_{2}[t]+\min \left\{q_{1}[t], T B r_{1}[t]\right\}-\min \left\{q_{2}[t], T B r_{2}[t]\right\} .
\end{aligned}
$$

The delay-outage constraint can be imposed as follows:

$$
\operatorname{Pr}\left(Q>Q^{\max }\right) \leq \zeta_{Q}
$$

Using (5.9), the delay-outage constraint (6.5) can be transformed into the following constraints in terms of arrival rate $\mu$ and power allocation variables:

$$
\mu T B \theta^{\mathrm{tar}}+\Omega_{i}\left(-\theta^{\mathrm{tar}}\right)=0, i=1,2
$$

where the delay exponent $\theta^{\text {tar }}$ is given by $(5.5) ; \Omega_{1}(\theta)$ and $\Omega_{2}(\theta)$ are the log moment generating functions (LMGFs) of the service processes of the source and relay queues, i.e.,:

$$
\Omega_{i}(\theta)=\log \mathbb{E}\left\{e^{\theta T B r_{i}[t]}\right\}, i=1,2
$$

\subsubsection{Problem Formulation}

Using (6.6) as the constraint transformation of (6.5), we formulate the power allocation problems with instantaneous CSIT or statistical CSIT.

With instantaneous CSIT, the B-FD-APA problem to maximize $\mu$ can be cast as follows:

$$
\begin{array}{cl}
\max _{\mu, P_{1}[t], P_{2}[t] \geq 0} & \mu \\
\text { s.t. : } & \mu T B \theta^{\mathrm{tar}}+\Omega_{i}\left(-\theta^{\mathrm{tar}}\right)=0, i=1,2, \\
& P_{1}[t]+P_{2}[t] \leq P^{\max }, \forall t,
\end{array}
$$

where $P^{\max }$ is the maximum total transmit power of the source and relay. 
On the other hand, with only statistical CSIT, the B-FD-SPA problem can be cast as:

$$
\begin{array}{ll}
\max _{\mu, P_{1}, P_{2} \geq 0} & \mu \\
\text { s.t.: } & \mu T B \theta^{\text {tar }}+\Omega_{i}\left(-\theta^{\text {tar }}\right)=0, i=1,2, \\
& P_{1}+P_{2} \leq P^{\max }
\end{array}
$$

Let $\mu_{\mathrm{B}-\mathrm{FD}-\mathrm{APA}}$, and $\mu_{\mathrm{B}-\mathrm{FD}-\mathrm{SPA}}$ denote the optimal values of (6.8) and (6.9), respectively, which are refereed as the effective capacities of the B-FD relaying under the corresponding power allocation schemes.

We now solve the optimization problems (6.8), and (6.9) in the following sections.

\subsection{Adaptive Power Allocation with Instantaneous CSIT}

\subsubsection{Optimal Solution}

Using (6.7) where $r_{i}[t], i=1,2$ are given by (6.2), the first set of constraints in (6.8) can be explicitly expressed as:

$$
\begin{aligned}
& \mu=-\frac{\Omega_{1}\left(-\theta^{\operatorname{tar}}\right)}{T B \theta^{\operatorname{tar}}}=-\frac{\log \mathbb{E}\left\{\left(1+\frac{h_{1}[t] P_{1}[t]}{\beta P_{2}[t]+1}\right)^{-\hat{\theta}}\right\}}{\hat{\theta} \log (2)} \\
& \mu=-\frac{\Omega_{2}\left(-\theta^{\operatorname{tar}}\right)}{T B \theta^{\operatorname{tar}}}=-\frac{\log \mathbb{E}\left\{\left(1+h_{2}[t] P_{2}[t]\right)^{-\hat{\theta}}\right\}}{\hat{\theta} \log (2)},
\end{aligned}
$$

where we denote the (normalized) delay exponent:

$$
\hat{\theta}=\theta^{\operatorname{tar}} T B / \log (2) .
$$


Substituting the latter expression in (6.10) for $\mu$ into the objective function, the problem (6.8) can be equivalently re-written in terms of power allocation variables as:

$$
\begin{aligned}
& \max _{\substack{P_{1}[t], P_{2}[t] \geq 0 \\
\text { s.t. : }}}-\frac{\log \mathbb{E}\left\{\left(1+h_{2}[t] P_{2}[t]\right)^{-\hat{\theta}}\right\}}{\hat{\theta} \log (2)} \\
& \frac{-\log \mathbb{E}\left\{\left(1+\frac{h_{1}[t] P_{1}[t]}{\beta P_{2}[t]+1}\right)^{-\hat{\theta}}\right\}}{\hat{\theta} \log (2)}=\frac{-\log \mathbb{E}\left\{\left(1+h_{2}[t] P_{2}[t]\right)^{-\hat{\theta}}\right\}}{\hat{\theta} \log (2)} \\
& P_{1}[t]+P_{2}[t] \leq P^{\max }, \forall t .
\end{aligned}
$$

The effective capacity $\mu_{\mathrm{B}-\mathrm{FD}-\mathrm{APA}}$ under a given delay constraint set by $\hat{\theta}$ is:

$$
\mu_{\mathrm{B}-\mathrm{FD}-\mathrm{APA}}=-\frac{\log \mathbb{E}\left\{\left(1+h_{2}[t] P_{2}^{*}[t]\right)^{-\hat{\theta}}\right\}}{\hat{\theta} \log (2)}
$$

where $P_{1}^{*}[t]$, and $P_{2}^{*}[t]$ is the optimal solution of $(6.12)$.

From the monotonic property of log function, the problem (6.12) is now equivalent to the following problem:

$$
\begin{gathered}
\min _{P_{1}[t], P_{2}[t] \geq 0} \mathbb{E}\left\{\left(1+h_{2}[t] P_{2}[t]\right)^{-\hat{\theta}}\right\} \\
\text { s.t.: } \\
\mathbb{E}\left\{\left(1+\frac{h_{1}[t] P_{1}[t]}{\beta P_{2}[t]+1}\right)^{-\hat{\theta}}\right\}=\mathbb{E}\left\{\left(1+h_{2}[t] P_{2}[t]\right)^{-\hat{\theta}}\right\} \\
P_{1}[t]+P_{2}[t] \leq P^{\max }, \forall t
\end{gathered}
$$

The equality constraint can be equivalently replaced by the smaller-or-equal inequality constraint. This is because at optimality, the inequality has to be satisfied with equality, otherwise, we can find another feasible solution with smaller objective function value.

To solve the constrained optimization problem (6.14), we employ Lagrangian approach. 
The (partial) Lagrangian of (6.14) is given by:

$$
\mathcal{L}=\mathbb{E}\{\mathcal{L}[t]\}
$$

where we have:

$$
\mathcal{L}[t]=(1-\omega)\left(1+h_{2}[t] P_{2}[t]\right)^{-\hat{\theta}}+\omega\left(1+\frac{h_{1}[t] P_{1}[t]}{\beta P_{2}[t]+1}\right)^{-\hat{\theta}}
$$

where $\omega \in(0,1)$ is the Lagrange multiplier associated with the first $\leq$ inequality constraint in (6.14).

We can see that, in order to minimize the Lagrangian $\mathcal{L}$ in $(6.15)$, the B-FD-APA solutions $P_{1}^{*}[t]$, and $P_{2}^{*}[t]$ are found to minimize $\mathcal{L}[t]$ in (6.16) in each frame $t$, i.e.,:

$$
\min _{P_{1}[t], P_{2}[t] \geq 0} \mathcal{L}[t] \quad \text { s.t. : } P_{1}[t]+P_{2}[t] \leq P^{\max } .
$$

Moreover, the multiplier $\omega$ is determined such that the following relationship holds at optimality:

$$
\mathbb{E}\left\{\left(1+\frac{h_{1}[t] P_{1}^{*}[t]}{\beta P_{2}^{*}[t]+1}\right)^{-\hat{\theta}}\right\}=\mathbb{E}\left\{\left(1+h_{2}[t] P_{2}^{*}[t]\right)^{-\hat{\theta}}\right\} .
$$

The following proposition is in order.

Proposition 6.1. The optimal B-FD-APA solution has $P_{1}^{*}[t]+P_{2}^{*}[t]=P^{\max }, \forall t$.

Proof. Since $\mathcal{L}[t]$ decreases with increasing $P_{1}[t]$, hence, at optimality, $P_{1}^{*}[t]+P_{2}^{*}[t]=P^{\max }$. Otherwise, we can find another feasible solution with smaller objective function value.

Using Proposition 6.1 , by substituting $P_{1}[t]=P^{\max }-P_{2}[t]$, the optimization problem (6.17) has single variable $P_{2}[t]$. Then, the optimal solution $P_{2}^{*}[t]$ (and $P_{1}^{*}[t]$ ) can be found using numerical search. We omit the details for brevity.

Depending on the instantaneous CSI $h_{1}[t]$ and $h_{2}[t]$, when the resulting $P_{1}^{*}[t]=P^{\max }$ or $P_{2}^{*}[t]=P^{\max }$, the relay actually operates in HD mode. In other words, the optimal

Note that $\omega$ cannot not be equal to zero or equal-or-larger than 1 , otherwise, we would have trivial solutions. 
B-FD-APA solution includes a built-in dynamic switching mechanism between HD and FD relaying as described in Algorithm 2.

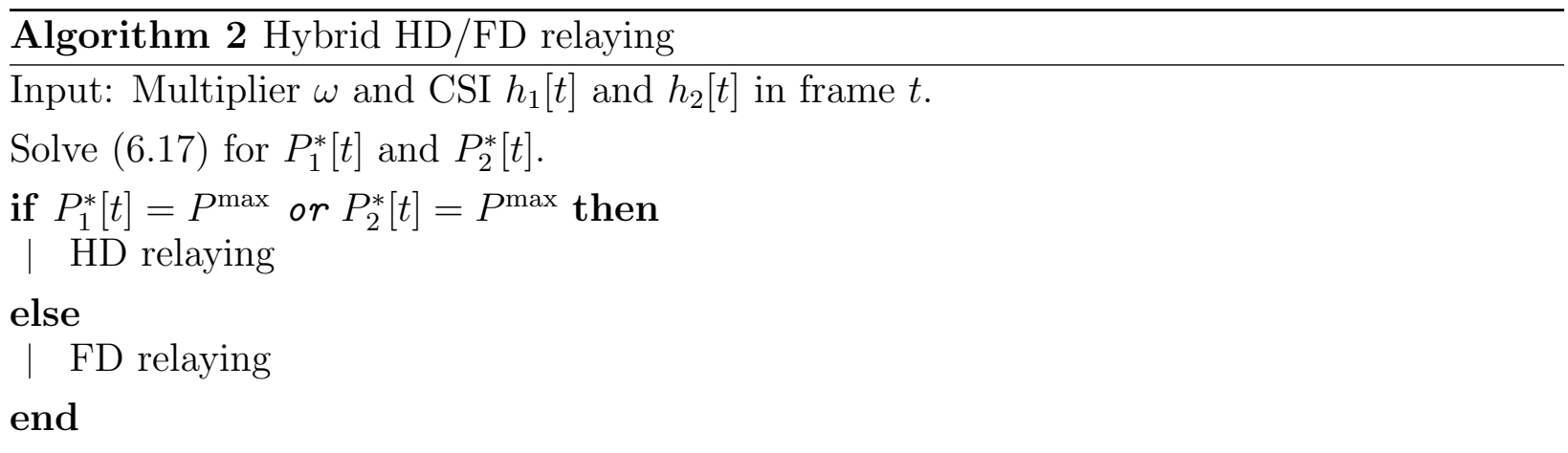

In the following, we study the optimal B-FD-APA solution under special cases on the delay constraint and SI cancellation parameter $\beta$.

\subsubsection{Special Cases}

\subsubsection{Very loose delay constraints}

When the delay constraint becomes very loose, i.e., $\zeta_{Q} \rightarrow 1$ (and $\hat{\theta} \rightarrow 0$ ), omitting the intermediate steps, we can show that the B-FD-APA problem in each frame $t$ can be written as follows:

$$
\begin{aligned}
& \max (1-\sigma) \log _{2}\left(1+h_{2}[t] P_{2}[t]\right)+\sigma \log _{2}\left(1+\frac{h_{1}[t] P_{1}[t]}{\beta P_{2}[t]+1}\right) \\
& \text { s.t.: } P_{1}[t]+P_{2}[t] \leq P^{\max } .
\end{aligned}
$$

The multiplier $\sigma \in(0,1)$ is determined such that:

$$
\mathbb{E}\left\{\log _{2}\left(1+\frac{h_{1}[t] P_{1}^{*}[t]}{\beta P_{2}^{*}[t]+1}\right)\right\}=\mathbb{E}\left\{\log _{2}\left(1+h_{2}[t] P_{2}^{*}[t]\right)\right\}
$$

The problem (6.19) is similar to the weighted sum-rate maximization problem [124]. We can see that in this case, the optimal B-FD-APA solution ensures equal average (ergodic) rates of the S-R and R-D links. 


\subsubsection{Very stringent delay constraints}

Under very stringent delay constraints, i.e., $\zeta_{Q} \rightarrow 0$ (and $\hat{\theta} \rightarrow \infty$ ), as seen from (6.13), the effective capacity $\mu_{\mathrm{B}-\mathrm{FD}-\mathrm{APA}}$ approaches 0 . It implies that B-FD relaying cannot support applications with very stringent delay constraints over fading channels. This result is not surprising since the delay-limited capacity of fading channels is shown to be zero.

\subsubsection{Zero residual SI}

Under the ideal FD assumption with zero residual SI, i.e., $\beta=0$, by omitting the intermediate steps, similar to (6.17), we have the following power allocation problem in each frame $t$ :

$$
\begin{array}{ll}
\min & (1-\xi)\left(1+h_{2}[t] P_{2}[t]\right)^{-\hat{\theta}}+\xi\left(1+h_{1}[t] P_{1}[t]\right)^{-\hat{\theta}} \\
\text { s.t.: } & P_{1}[t]+P_{2}[t] \leq P^{\max },
\end{array}
$$

where $\xi \in(0,1)$ is the multiplier which is determined such that:

$$
\mathbb{E}\left\{\left(1+h_{1}[t] P_{1}^{*}[t]\right)^{-\hat{\theta}}\right\}=\mathbb{E}\left\{\left(1+h_{2}[t] P_{2}^{*}[t]\right)^{-\hat{\theta}}\right\}
$$

It can be seen that the problem (6.21) is a convex optimization problem by verifying the non-negativeness of the second-order derivative of the objective function. Using Lagrangian approach, we can differentiate the objective function in (6.21), and set the resulting equations to zero, and accounting for the non-negativeness of the power allocation solution, we can derive the optimal solution with zero residual SI as:

$$
P_{1}^{*}[t]=\frac{1}{h_{1}[t]}\left[\left(\frac{\xi \hat{\theta} h_{1}[t]}{\eta[t]}\right)^{1 /(\hat{\theta}+1)}-1\right]^{+}
$$

and

$$
P_{2}^{*}[t]=\frac{1}{h_{2}[t]}\left[\left(\frac{(1-\xi) \hat{\theta} h_{2}[t]}{\eta[t]}\right)^{1 /(\hat{\theta}+1)}-1\right]^{+},
$$

where the multiplier $\eta[t]>0$ is determined such that:

$$
P_{1}^{*}[t]+P_{2}^{*}[t]=P^{\max }
$$




\subsubsection{Very large SI cancellation parameter $\beta$}

For simplicity, consider the unconstrained delay case with the optimization problem (6.19). It can be observed that for large $\beta$, any positive $P_{2}[t]$ will result in large INR, which then significantly reduces the rate of the $\mathrm{S}-\mathrm{R}$ link if the source is transmitting. It implies that FD relaying mode is not beneficial. Mathematically, the optimal solution of (6.19) mostly prescribes either $P_{1}^{*}[t]$ or $P_{2}^{*}[t]$ being positive depending on $h_{1}[t]$ and $h_{2}[t]$. It implies that when the SI cancellation quality is very bad, B-FD-APA approaches B-HD-ALS relaying [108]. This finding will be demonstrated in the illustrative examples in Section VI.

\subsubsection{Iterative Power Allocation Algorithm}

The optimal B-FD-APA solution depends on the multiplier $\omega$, which satisfies (6.18). Numerical approaches can be used to determine $\omega$, which require the fading statistics to be known. When the fading statistics are unknown, which is common in real-life communications, we can utilize the following iterative algorithm. We initialize the multiplier with $\omega[1] \in(0,1)$. Then, in transmission frame $t=1,2, \ldots$, we carry out the following update:

$$
\begin{aligned}
\omega[t+1]=[\omega[t] & +\epsilon[t]\left(\left(1+\frac{h_{1}[t] P_{1}^{*}[t]}{\beta P_{2}^{*}[t]+1}\right)^{-\hat{\theta}}\right. \\
& \left.\left.-\left(1+h_{2}[t] P_{2}^{*}[t]\right)^{-\hat{\theta}}\right)\right]_{\varsigma}^{1-\varsigma}
\end{aligned}
$$

where $[x]_{a}^{b}$ denotes the projection of $x$ on the interval $[a, b]$ for $a \leq b$ and $\varsigma$ is some small positive number. The decreasing positive sequence $\epsilon[t]$ that dictates the convergence speed, satisfies:

$$
\sum_{t=1}^{\infty} \epsilon[t]=\infty ; \quad \sum_{t=1}^{\infty}(\epsilon[t])^{2}<\infty .
$$

The power allocation $P_{1}^{*}[t]$, and $P_{2}^{*}[t]$ in frame $t$ is computed from (6.17) using the current estimate $\omega[t]$. The iterative update (6.26) converges, and satisfies the equality constraint (6.14) at convergence. The algorithm does not assume any specification on the fading statistics. 


\subsection{Static Power Allocation with Statistical CSIT}

We recall that implementing B-FD-APA requires instantaneous CSIT. However, in reallife system, instantaneous CSIT may be unavailable due to high signaling complexity for CSI feedback from the receivers. In such scenario, the source and relay should transmit with constant powers. Moreover, statistical information about the channels, can always be accessible to the transmitters, e.g., statistical CSIT, since the duration over which channel fading processes are stationary is several orders of magnitude longer than the duration of the fades. Exploiting the statistical CSIT, we can optimize source and relay power allocation for source arrival rate maximization, i.e., the B-FD-SPA problem (6.9). In the following, we derive the optimal solution of (6.9) and study its properties.

\subsubsection{Optimal Solution}

Consider static source and relay power allocation $P_{1}$, and $P_{2}$, respectively. In the following, for the sake of clarity, we explicitly express the LMGFs of the service processes of the source and relay queues in (6.7) as functions of (normalized) delay exponent $\theta, P_{1}$, and $P_{2}$ as follows:

$$
\begin{aligned}
& \Omega_{1}\left(\theta, P_{1}, P_{2}\right)=\log \mathbb{E}\left\{\left(1+\frac{h_{1}[t] P_{1}}{\beta P_{2}+1}\right)^{\theta}\right\}, \\
& \Omega_{2}\left(\theta, P_{2}\right)=\log \mathbb{E}\left\{\left(1+h_{2}[t] P_{2}\right)^{\theta}\right\} .
\end{aligned}
$$

Using the above notations, the B-FD-SPA problem (6.9) can be re-written as:

$$
\begin{array}{cl}
\max _{\mu, P_{1}, P_{2} \geq 0} \mu \text { s.t.: } \quad \mu \hat{\theta} \log (2)+\Omega_{1}\left(-\hat{\theta}, P_{1}, P_{2}\right)=0 \\
& \mu \hat{\theta} \log (2)+\Omega_{2}\left(-\hat{\theta}, P_{2}\right)=0 \\
& P_{1}+P_{2} \leq P^{\max }
\end{array}
$$

where $\hat{\theta}$ is given by (6.11). From the first two constraints in (6.28), we have:

$$
\mu=-\frac{\Omega_{1}\left(-\hat{\theta}, P_{1}, P_{2}\right)}{\hat{\theta} \log (2)}, \quad \mu=-\frac{\Omega_{2}\left(-\hat{\theta}, P_{2}\right)}{\hat{\theta} \log (2)} .
$$


Substituting the latter expression in (6.29) for $\mu$ into the objective function, and after some simple manipulations, the optimal B-FD-SPA solution $P_{1}^{*}$, and $P_{2}^{*}$ of $(6.28)$ can be shown to be the solution of the following problem:

$$
\min _{P_{1}, P_{2} \geq 0} \Omega_{2}\left(-\hat{\theta}, P_{2}\right) \text { s.t. : } \begin{gathered}
\Omega_{1}\left(-\hat{\theta}, P_{1}, P_{2}\right)=\Omega_{2}\left(-\hat{\theta}, P_{2}\right), \\
P_{1}+P_{2} \leq P^{\max }
\end{gathered}
$$

The following proposition is needed.

Proposition 6.2. $\Omega_{1}\left(-\theta, P_{1}, P_{2}\right)$ decreases with increasing $P_{1}$, and increases with increasing $P_{2} . \Omega_{2}\left(-\theta, P_{2}\right)$ decreases with increasing $P_{2}$.

Proof. Proposition 6.2 follows directly from the expressions in (6.27).

Theorem 6.1. The effective capacity of B-FD-SPA is given by:

$$
\mu_{\mathrm{B}-\mathrm{FD}-\mathrm{SPA}}=-\Omega_{2}\left(-\hat{\theta}, P_{2}^{*}\right) /(\hat{\theta} \log (2))
$$

where the optimal B-FD-SPA solution $P_{1}^{*}=P^{\max }-P_{2}^{*}$, and $P_{2}^{*} \in\left(0, P^{\max }\right)$ uniquely satisfies:

$$
\Omega_{1}\left(-\hat{\theta}, P^{\max }-P_{2}^{*}, P_{2}^{*}\right)=\Omega_{2}\left(-\hat{\theta}, P_{2}^{*}\right)
$$

Proof. First, we prove that $P_{1}^{*}$, and $P_{2}^{*}$ satisfy $P_{1}^{*}+P_{2}^{*}=P^{\max }$ by contradiction. Suppose $P_{1}^{*}+P_{2}^{*}<P^{\max }$, and from $(6.30), \Omega_{2}\left(-\hat{\theta}, P_{2}^{*}\right)$ is minimized.

Consider another feasible solution $P_{1}^{\dagger}$, and $P_{2}^{\dagger}$ such that $P_{1}^{\dagger}>P_{1}^{*}, P_{2}^{\dagger}>P_{2}^{*}, P_{1}^{\dagger}+P_{2}^{\dagger}=$ $P^{\max }$, and, by Proposition 2,

$$
\Omega_{1}\left(-\hat{\theta}, P_{1}^{\dagger}, P_{2}^{\dagger}\right)=\Omega_{2}\left(-\hat{\theta}, P_{2}^{\dagger}\right)
$$

Hence, we have $\Omega_{2}\left(-\hat{\theta}, P_{2}^{\dagger}\right)<\Omega_{2}\left(-\hat{\theta}, P_{2}^{*}\right)$ by Proposition 2, contradicting our assumption that $\Omega_{2}\left(-\hat{\theta}, P_{2}^{*}\right)$ is minimized. We conclude that at optimality, $P_{1}^{*}+P_{2}^{*}=P^{\max }$.

By substituting $P_{1}^{*}=P^{\max }-P_{2}^{*}$ in the equality constraint of (6.30), we obtain (6.31). Moreover, using the monotonicity of the functions in Proposition 6.2, the solution $P_{2}^{*} \in$ $\left(0, P^{\max }\right)$ satisfying $(6.31)$ is unique. 
To obtain more insights, we study several properties of the optimal B-FD-SPA solution in Theorem 6.1.

\subsubsection{Properties of the Optimal Solution}

Proposition 6.3. The optimal B-FD-SPA solution $P_{1}^{*}$, and $P_{2}^{*}$ increases with increasing $P^{\max }$.

Proof. Notice that while $\Omega_{1}\left(-\hat{\theta}, P^{\max }-P_{2}, P_{2}\right)$ decreases with $P^{\max }$ and increases with $P_{2}$, $\Omega_{2}\left(-\hat{\theta}, P_{2}\right)$ decreases with $P_{2}$. Hence, when $P^{\max }$ increases, $P_{2}^{*}$ increases in order to satisfy (6.31). That $P_{1}^{*}$ increases with increasing $P^{\max }$ can be argued analogously.

A consequence of Proposition 6.3 is that $\mu_{\mathrm{B}-\mathrm{FD}-\mathrm{SPA}}$ increases with increasing $P^{\mathrm{max}}$ as expected.

Proposition 6.4. The optimal relay power allocation $P_{2}^{*}$ decreases with increasing $\beta$.

Proof. Let us define the following function:

$$
g\left(P_{2}, \beta\right)=\Omega_{1}\left(-\hat{\theta}, P^{\max }-P_{2}, P_{2}\right)-\Omega_{2}\left(-\hat{\theta}, P_{2}\right),
$$

By Proposition 6.2, we can see that $g\left(P_{2}, \beta\right)$ increases with increasing $P_{2}$. Moreover, $g\left(P_{2}, \beta\right)$ increases with increasing $\beta$. Hence, as $\beta$ increases, $P_{2}=P_{2}^{*}$ decreases in order to satisfy $g\left(P_{2}^{*}, \beta\right)=0$.

Proposition 6.4 indicates that for larger $\beta$, less power is allocated to the relay to avoid excessively large INR. A consequence of Proposition 6.4 is that $\mu_{\mathrm{B}-\mathrm{FD}-\mathrm{SPA}}$ decreases under worse SI cancellation quality.

Proposition 6.5. When the links have similar fading distributions, the optimal relay power allocation $P_{2}^{*}$ satisfies:

$$
\frac{\mathbb{E}\left\{h_{1}[t]\right\}\left(P^{\max }-P_{2}^{*}\right)}{\beta P_{2}^{*}+1}=\mathbb{E}\left\{h_{2}[t]\right\} P_{2}^{*}
$$

Proof. From (6.27), we can see that in order to satisfy (6.31), the links have to have equal average signal strengths. Hence, (6.33) follows because the terms in the left side, and right side are the average SINR of the S-R link and average SNR of the R-D link, respectively. 
Proposition 6.5 implies that when the links have similar fading distributions, the optimal B-FD-SPA solution is independent of the delay constraint.

\subsubsection{Special Cases}

We study the B-FD-SPA solution for various special cases on the delay constraint and SI cancellation parameter $\beta$.

\subsubsection{Very loose delay constraints}

Proposition 6.6. Under very loose delay constraints, i.e., $\hat{\theta} \rightarrow 0$, the optimal relay power allocation $P_{2}^{*}$ satisfies:

$$
\mathbb{E}\left\{\log _{2}\left(1+\frac{h_{1}[t]\left(P^{\max }-P_{2}^{*}\right)}{\beta P_{2}^{*}+1}\right)\right\}=\mathbb{E}\left\{\log _{2}\left(1+h_{2}[t] P_{2}^{*}\right)\right\}
$$

Proof. Dividing both sides of (6.31) by $\hat{\theta} \log (2)$ and then taking the limits of both sides when $\hat{\theta} \rightarrow 0$ using L'Hopital rule, we obtain (6.34).

Proposition 6.6 implies that under very loose delay constraints, the optimal B-FD-SPA solution guarantees equal ergodic rates of the S-R and R-D links, which is similar to the case of B-FD-APA.

\subsubsection{Very stringent delay constraints}

Under very stringent delay constraints, similar to the case of B-FD-APA, the effective capacity $\mu_{\mathrm{B}-\mathrm{FD}-\mathrm{SPA}}$ approaches 0 .

\subsubsection{Zero residual SI}

Under zero residual SI assumption, from (6.27), the LMGF of the service process of the source queue is:

$$
\Omega_{1}^{\text {Ideal }}\left(\theta, P_{1}\right)=\log \mathbb{E}\left\{\left(1+h_{1}[t] P_{1}\right)^{\theta}\right\} .
$$

The LMGF of the service process of the relay queue remains $\Omega_{2}\left(\theta, P_{2}\right)$ in (6.27). 
Proposition 6.7. The effective capacity of the B-FD-SPA with zero residual SI is given by:

$$
\mu_{\mathrm{B}-\mathrm{FD}-\mathrm{SPA}}^{\mathrm{Ideal}}=-\Omega_{2}\left(-\hat{\theta}, P_{2}^{\ddagger}\right) /(\hat{\theta} \log (2))
$$

with optimal power allocation $P_{1}^{\ddagger}=P^{\max }-P_{2}^{\ddagger}$, and $P_{2}^{\ddagger} \in\left(0, P^{\max }\right)$ uniquely satisfies:

$$
\Omega_{1}^{\text {Ideal }}\left(-\hat{\theta}, P^{\max }-P_{2}^{\ddagger}\right)=\Omega_{2}\left(-\hat{\theta}, P_{2}^{\ddagger}\right) .
$$

Proof. Proposition 6.7 can be viewed as a special case of Theorem 6.1 when the SI cancellation parameter $\beta=0$.

\subsubsection{Very large SI cancellation parameter $\beta$}

For large $\beta$, the optimal relay power allocation $P_{2}^{*}$ satisfying (6.31) becomes small and approaches 0 (due to Proposition 6.4). Hence, $\mu_{\mathrm{B}-\mathrm{FD}-\mathrm{SPA}}$ approaches 0.

We can see the benefits of B-FD-APA over B-FD-SPA. For large INR, while $\mu_{\mathrm{B}-\mathrm{FD}-\mathrm{APA}}$ approaches the effective capacity of $\mathrm{HD}$ relaying, $\mu_{\mathrm{B}-\mathrm{FD}-\mathrm{SPA}}$ approaches 0 . This fact will be demonstrated in the numerical studies in Section VI.

\subsection{Power Allocation in Non-Buffer Full-Duplex Relaying}

In this section, we deal with power allocation and effective capacity analysis for non-buffer FD (N-FD) relaying, which was not reported in existing literature. In N-FD relaying, the relay forwards the received packets to the destination immediately without buffering them, i.e., the queue $Q_{2}$ in Figure 6.1 does not exist. Hence, the delay-outage constraint (6.5) for this case is described as follows:

$$
\operatorname{Pr}\left(Q_{1}>Q^{\max }\right) \leq \zeta_{Q}
$$

For decode-and-forward relaying scheme, the equivalent end-to-end rate in frame $t$ is equal to the minimum of the rates of the S-R and R-D links [52], [53]:

$$
r[t]=\min \left\{r_{1}[t], r_{2}[t]\right\},
$$


where $r_{1}[t], r_{2}[t]$ are given in (6.2) (for adaptive power allocation) or (6.3) (for static power allocation). The effective capacity of the N-FD relaying can be shown to be [32]:

$$
\mu_{\mathrm{N}-\mathrm{FD}}=-\frac{1}{\bar{\theta}_{1}} \log \mathbb{E}\left\{e^{-\bar{\theta}_{1} r[t]}\right\}
$$

where the (normalized) delay exponent $\bar{\theta}_{1}$ can be derived from $(6.36)$ as:

$$
\bar{\theta}_{1}=-T B \log \left(\zeta_{Q}\right) / Q_{\max }
$$

The power allocation problems for N-FD relaying are described next.

\subsubsection{Adaptive Power Allocation}

With instantaneous CSIT, the N-FD relaying with adaptive power allocation (N-FD-APA) problem can be cast as:

$$
\max _{P_{1}[t], P_{2}[t] \geq 0} \mu_{\mathrm{N}-\mathrm{FD}} \quad \text { s.t. : } P_{1}[t]+P_{2}[t] \leq P^{\max } .
$$

It can be seen that the optimal N-FD-APA solution $P_{1}^{\star}[t]$, and $P_{2}^{\star}[t]$ of (6.40) should ensure $r_{1}[t]=r_{2}[t]$ in each frame $t$. Also, it must be true that $P_{1}^{\star}[t]+P_{2}^{\star}[t]=P^{\max }$. Hence, we have:

$$
h_{2}[t] P_{2}^{\star}[t]=\frac{h_{1}[t]\left(P^{\max }-P_{2}^{\star}[t]\right)}{\beta P_{2}^{\star}[t]+1} .
$$

Then, we can solve for $P_{1}^{\star}[t]$, and $P_{2}^{\star}[t]$. Let $\mu_{\mathrm{N}-\mathrm{FD}-\mathrm{APA}}$ denote the optimal value of (6.40). We can see that when $\beta$ becomes large, the end-to-end rate $r[t]$ becomes small, and hence, $\mu_{\mathrm{N}-\mathrm{FD}-\mathrm{APA}}$ becomes small and approaches 0 .

\subsubsection{Static Power Allocation}

With statistical CSIT, the N-FD relaying with static power allocation (N-FD-SPA) problem can be expressed as:

$$
\max _{P_{1}, P_{2} \geq 0} \mu_{\mathrm{N}-\mathrm{FD}} \quad \text { s.t. }: P_{1}+P_{2} \leq P^{\max } .
$$

The optimal N-FD-SPA solution $P_{1}^{\star}$, and $P_{2}^{\star}$ should satisfy $P_{1}^{\star}+P_{2}^{\star}=P^{\max }$. Let $\mu_{\mathrm{N}-\mathrm{FD}-\mathrm{SPA}}$ denote the optimal value of (6.41). 
From (6.37), we have:

$$
r[t]=\log _{2}\left(1+\min \left\{\operatorname{sinr}_{1}[t], \operatorname{snr}_{2}[t]\right\}\right) .
$$

Consider the case of Rayleigh fading links. Consequently, from (6.3), we have $\operatorname{sinr}_{1}[t]$, and $\operatorname{snr}_{2}[t]$ are exponential random variables with hazard rates $z_{1}$, and $z_{2}$, respectively, where:

$$
z_{1}=\frac{\beta P_{2}+1}{\mathbb{E}\left\{h_{1}[t]\right\} P_{1}} ; \quad z_{2}=\frac{1}{\mathbb{E}\left\{h_{2}[t]\right\} P_{2}}
$$

Hence, the random variable $\min \left\{\operatorname{sinr}_{1}[t], \operatorname{snr}_{2}[t]\right\}$ is also exponentially distributed with hazard rate $z=z_{1}+z_{2}$ and mean $1 / z$. Hence, in order to maximize $\mu_{\mathrm{N}-\mathrm{FD}}$, we need to maximize $1 / z$, and equivalently, minimize $z$. Hence, the optimal $P_{2}^{\star}$ for N-FD-SPA can be found as the optimal solution of the following problem:

$$
\min _{P_{2} \geq 0} \frac{\beta P_{2}+1}{\mathbb{E}\left\{h_{1}[t]\right\}\left(P^{\max }-P_{2}\right)}+\frac{1}{\mathbb{E}\left\{h_{2}[t]\right\} P_{2}} .
$$

By differentiating the objective function and setting the resulting expression to 0 , we can solve for $P_{2}^{\star}$.

\subsection{Illustrative Results and Discussions}

\subsubsection{Settings}

We consider a decode-and-forward S-R-D link as shown in Fig. 6.1 where the relay is assumed to be in the middle of the source and the destination with equal S-R and R-D distances. As previously mentioned, the path loss (including both transmit and receive antenna gains) is normalized to 1 in both S-R link and R-D link. We assume Rayleigh fading links with average channel power gains $\mathbb{E}\left\{h_{1}[t]\right\}=\mathbb{E}\left\{h_{2}[t]\right\}=\mathbb{E}\{h\}=0 \mathrm{~dB}$, block-fading duration $T$, and bandwidth $B$. Hence, the average SINR of the S-R link, and average SNR of the R-D link are $P_{1} /\left(\beta P_{2}+1\right)$, and $P_{2}$, respectively. Also, $\beta P_{2}$ represents the INR. For the delay constraint (6.5), we fix $Q^{\max }=3000$.

In the following, we illustrate the effects of the SI cancellation quality (by varying $\beta$ ), the total power constraint (by varying $P^{\max }$ ), and the delay constraint (by varying queuelength-outage probability $\zeta_{Q}$ ) on the performance of the various relaying schemes including 
the proposed B-FD, and N-FD relaying schemes (B-FD-APA, B-FD-SPA, N-FD-APA, NFD-SPA), and existing B-HD relaying schemes. Recall that, in HD relaying, the relay can only transmit or receive during a frame. With statistical CSIT, [79] proposed and analyzed the effective capacity of a B-HD relaying with fixed link scheduling (B-HD-FLS), where the source and relay alternatively transmit over transmission frames independently of the CSI. With instantaneous CSIT, [108] studied the B-HD relaying with adaptive link scheduling (B-HD-ALS) under delay-outage constraint. In each frame, depending on $h_{1}[t]$ and $h_{2}[t]$, either the S-R link or the R-D link is selected to be active.

For fair comparisons, all the transmission schemes have similar total power and delay constraints.

\subsubsection{Effects of SI Cancellation}

We first investigate the optimal static power allocation when $\beta$ varies. The results are displayed in Fig. 6.2 for $P^{\max }=20 \mathrm{~dB}$, and $10 \mathrm{~dB}$. Under the assumptions of Rayleigh fading links with $\mathbb{E}\left\{h_{1}[t]\right\}=\mathbb{E}\left\{h_{2}[t]\right\}=1$, from (6.33) and (6.43), we can see that B-FDSPA and N-FD-SPA offer the same optimal solutions, that are independent of the delay constraint. We can verify that $P_{1}^{*}+P_{2}^{*}=P^{\max }$ as expected. For a given $\beta, P_{1}^{*}$, and $P_{2}^{*}$ increase with increasing $P^{\max }$ as stated in Proposition 6.3. Also, due to non-zero residual SI, $P_{2}^{*}$ is always smaller than $P_{1}^{*}$, i.e., for ideal $\mathrm{FD}$ with $\beta=0, P_{1}^{*}=P_{2}^{*}$, and for a given $P^{\max }$, $P_{2}^{*}$ decreases (and $P_{1}^{*}$ increases) with increasing $\beta$ (i.e., worse SI cancellation) to reduce the resulting INR $\beta P_{2}^{*}$ as expected by Proposition 6.4.

With the total power $P^{\max }=20 \mathrm{~dB}$, Figures 6.3 and 6.4 plot the (effective) capacities of the relaying schemes versus $\beta$ for two values of $\zeta_{Q}=1$, and $10^{-4}$, respectively. Notice that the performances of the HD relaying schemes are independent of $\beta$. In both figures, we can observe that the capacities of the FD relaying schemes decrease with increasing $\beta$ as expected.

Consider the case of unconstrained delay, i.e., $\zeta_{Q}=1$. With instantaneous CSIT, BFD-APA always outperforms B-HD-ALS, which is expected as we mentioned previously, the latter can be considered as a special case of the former. For instance, for $\beta=.03$, and .1 , B-FD-APA achieves $129.8 \%$, and $117.9 \%$, respectively, higher capacity than B-HD-ALS. When $\beta$ becomes large, B-FD-APA approaches B-HD-ALS as mentioned in Section III.C. In fact, B-HD-ALS can be viewed as a special case of the proposed B-FD-APA when only 


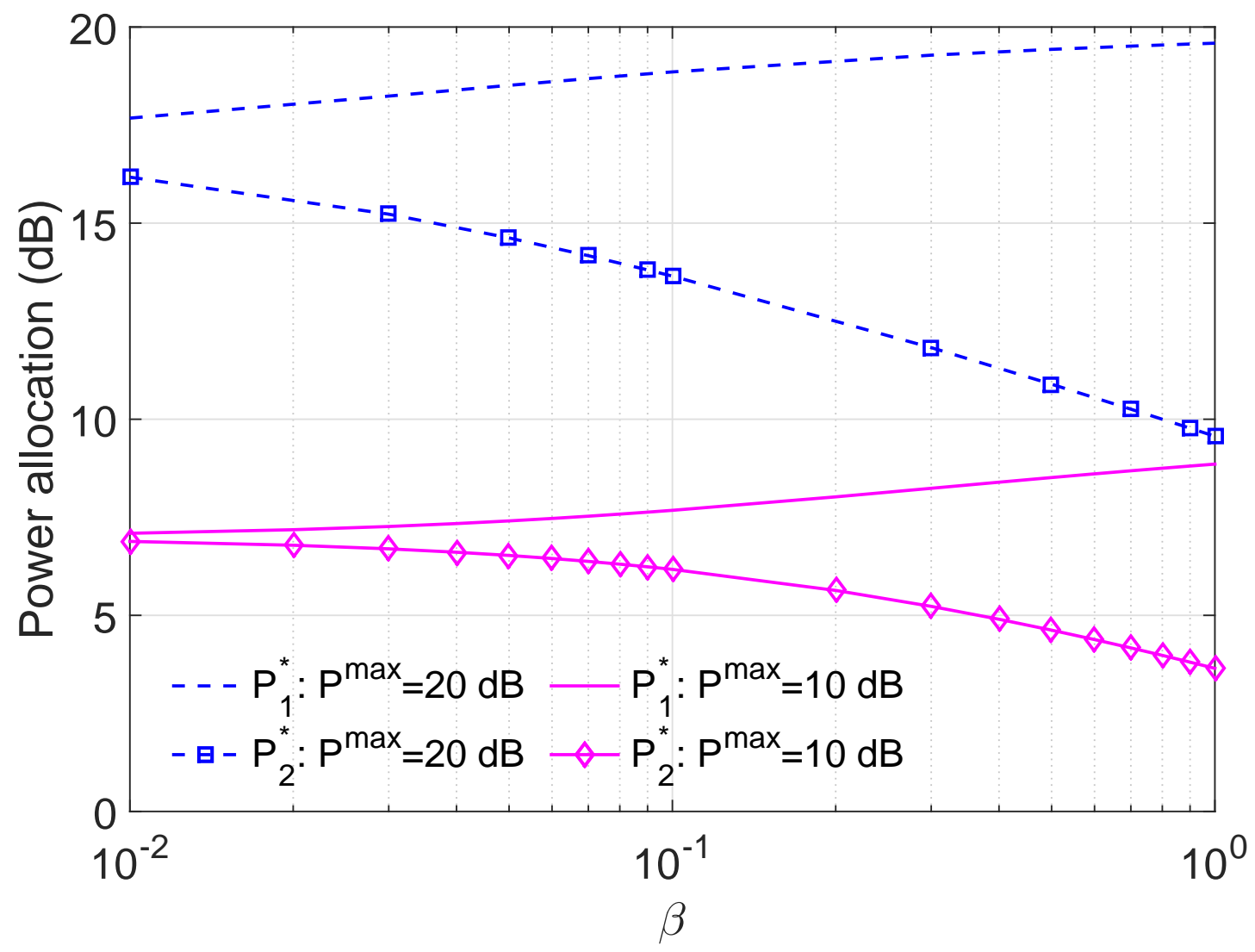

Fig. 6.2: Optimal static power allocation versus $\beta$.

either $P_{1}[t]$ or $P_{2}[t]$ can be non-zero (and equal to $P^{\max }$ ) in each frame $t$. Also, B-FDAPA attains higher capacity than N-FD-APA for all values of $\beta$. With statistical CSIT, similar observations regarding the performances of the relaying schemes as in the case of instantaneous CSIT can be made. For $\beta=.01$, and 0.1 , B-FD-SPA attains $159.2 \%$ and $133.5 \%$, respectively, higher capacity than B-HD-FLS. However, while B-FD-APA always outperforms B-HD-ALS for all $\beta$, B-FD-SPA outperforms B-HD-FLS only under sufficiently small $\beta$, i.e., $\beta \leq \sim .75$ for the considered example. The reason is that for large $\beta$, the signal qualities of the S-R and R-D links are severely degraded under B-FD-SPA relaying since FD relaying mode is always employed. Furthermore, from Fig. 6.3, we can observe the gain of adaptive power allocation (APA) over static power allocation (SPA) in B-FD relaying increasing with $\beta$. For small $\beta$, the gain is marginal. This implies that we might not need 
6 Power Allocation for Buffer-Aided Full-Duplex Relaying with Imperfect

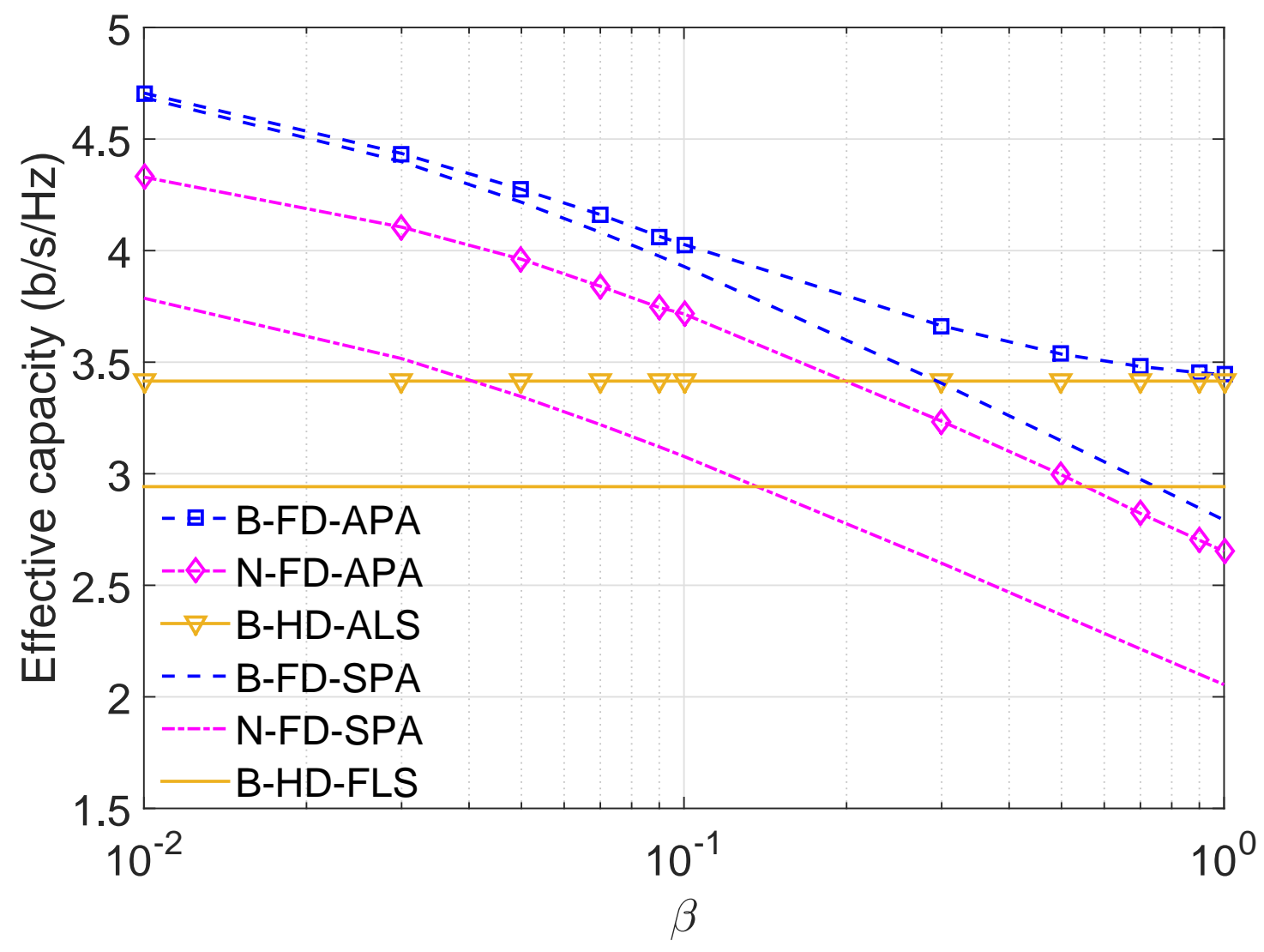

Fig. 6.3: Effective capacity versus $\beta$ with $\zeta_{Q}=1$. 
instantaneous CSIT when the SI cancellation is good. In other words, good SI cancellation can save CSI feedback complexity.

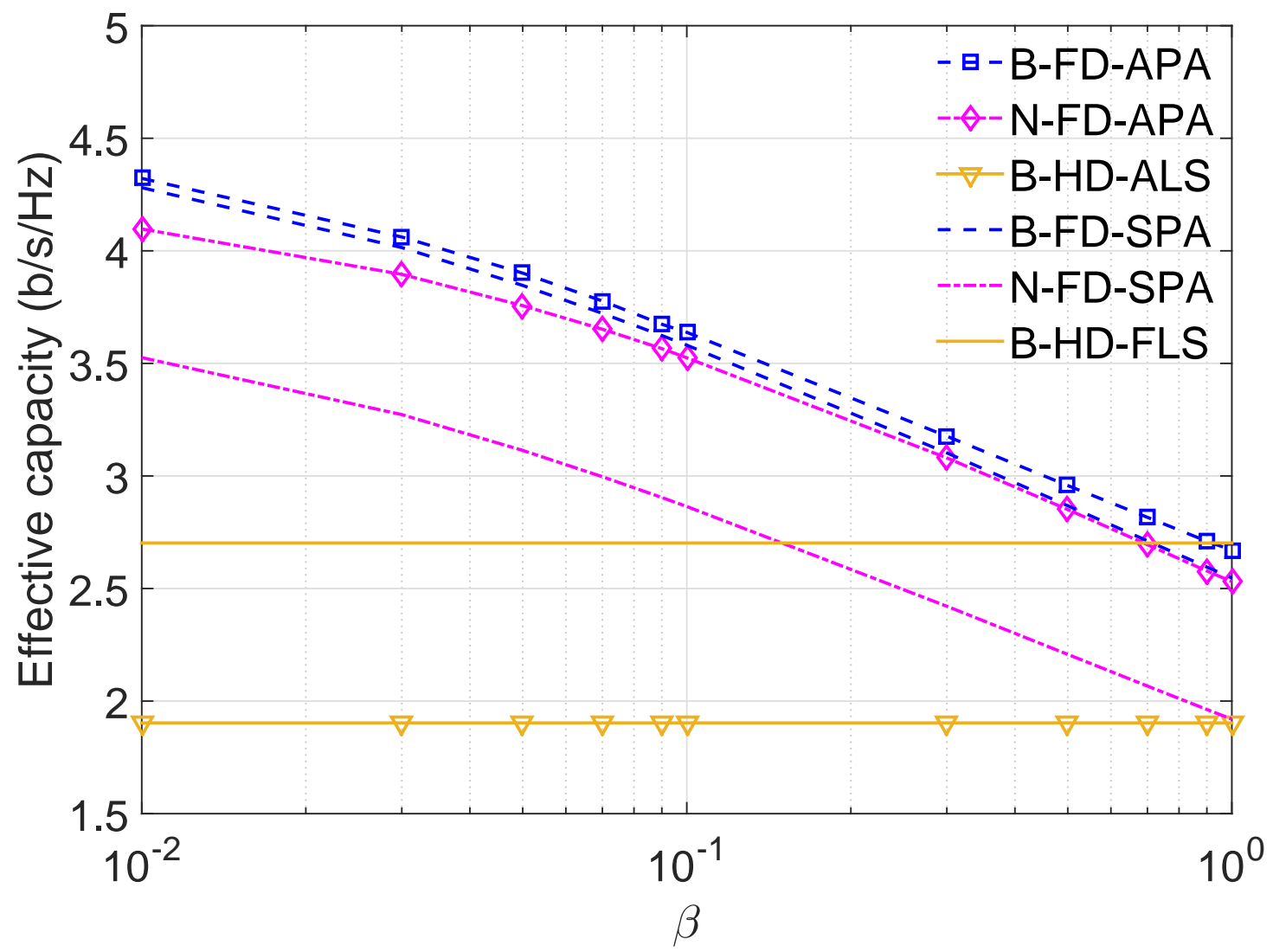

Fig. 6.4: Effective capacity versus $\beta$ with $\zeta_{Q}=10^{-4}$.

Now, consider the case of stringent delay constraint with $\zeta_{Q}=10^{-4}$ in Fig. 6.4. In this case, B-HD-FLS performs better than B-HD-ALS as mentioned previously. We can see that B-FD-SPA attains higher capacity than B-HD-FLS when $\beta$ is sufficiently small. However, unlike in the case $\zeta_{Q}=1$, B-FD-APA can be worse than B-HD-FLS for sufficiently large $\beta$. Under such setting, while the data needs to be transmitted soon to the destination, FD relaying mode is preferred, but still inefficient (due to large $\beta$ ). Moreover, the capacity gain of B-FD-APA over B-FD-SPA for $\zeta_{Q}=10^{-4}$ is decreased as compared with the unconstrained delay case. This is because the capacity is dominated by the (stringent) delay constraints, and hence, adaptive power allocation to increase the instantaneous rates become less beneficial. 


\subsubsection{Effects of Total Power Constraint}

In this section, we fix the SI cancellation parameter $\beta=.1$, and investigate the performance of various transmission schemes when the total power $P^{\max }$ varies.

For comparison, we also include the direct transmission (DT) from source (S) to (D), i.e., without relay (D) in Fig. 6.1. In this case, the source transmits with power $P^{\max }$ in each frame. The effective capacity of the direct S-D link under delay constraint (6.5) is given as:

$$
\mu_{\mathrm{DT}}=-\frac{1}{\bar{\theta}_{1}} \log \mathbb{E}\left\{\left(1+P^{\max } h_{\mathrm{SD}}[t]\right)^{-\bar{\theta}_{1} / \log (2)}\right\}
$$

where $\bar{\theta}_{1}$ is given by (6.39); $h_{\mathrm{SD}}[t]$ is the channel power gain of the S-D link in frame $t$ with mean $\mathbb{E}\left\{h_{\mathrm{SD}}[t]\right\}$. We also assume Rayleigh fading S-D link. Due to our assumptions that the $\mathrm{S}-\mathrm{D}$ distance is twice the $\mathrm{S}-\mathrm{R}$ (or R-D) distance, we have $h_{\mathrm{SD}}[t]$ is exponentially distributed with $\mathbb{E}\left\{h_{\mathrm{SD}}[t]\right\}=\mathbb{E}\left\{h_{1}\right\} / 2^{\gamma}=\mathbb{E}\left\{h_{2}\right\} / 2^{\gamma}$, where $\gamma$ is the path-loss exponent. In the following, we choose $\gamma=2$ for illustrative purpose.

Figures 6.5 and 6.6 plot the capacities of the relaying schemes and DT scheme versus $P^{\max }$ for two values of $\zeta_{Q}=1$, and $10^{-4}$, respectively.

It can be observed that B-FD relaying outperforms B-HD relaying and N-FD relaying under similar CSIT availability assumption. The gain of B-FD relaying over B-HD relaying increases with increasing $P^{\text {max }}$, implying the advantages of the former in high SNR region. For small $P^{\text {max }}$, while the gaps between B-FD-APA and B-HD-ALS are small, the gaps between B-FD-SPA and B-HD-FLS are more significant. This demonstrates the benefits of B-FD relaying with statistical CSIT in low SNR region. In comparing with DT mode, we can see that B-FD relaying outperforms DT when $P^{\max }$ is sufficiently small. This is expected since, in general, relaying is preferable to DT mode when the direct link power is weak. Moreover, the gaps between B-FD-APA and B-FD-SPA remain almost constant for all $P^{\max }$ and are smaller under more stringent delay constraints (Fig. 6.6). Hence, the choice between B-FD-APA and B-FD-SPA depends very much on the trade-off between the required effective capacity and the CSI feedback complexity/costs.

To provide more insights into the performances of various transmission schemes, we consider the statistical CSIT case with $\zeta_{Q}=1$ for simplicity. Assume total power $P^{\text {max }}$, SI cancellation parameter $\beta$ with corresponding B-FD-SPA and N-FD-SPA power allocation 


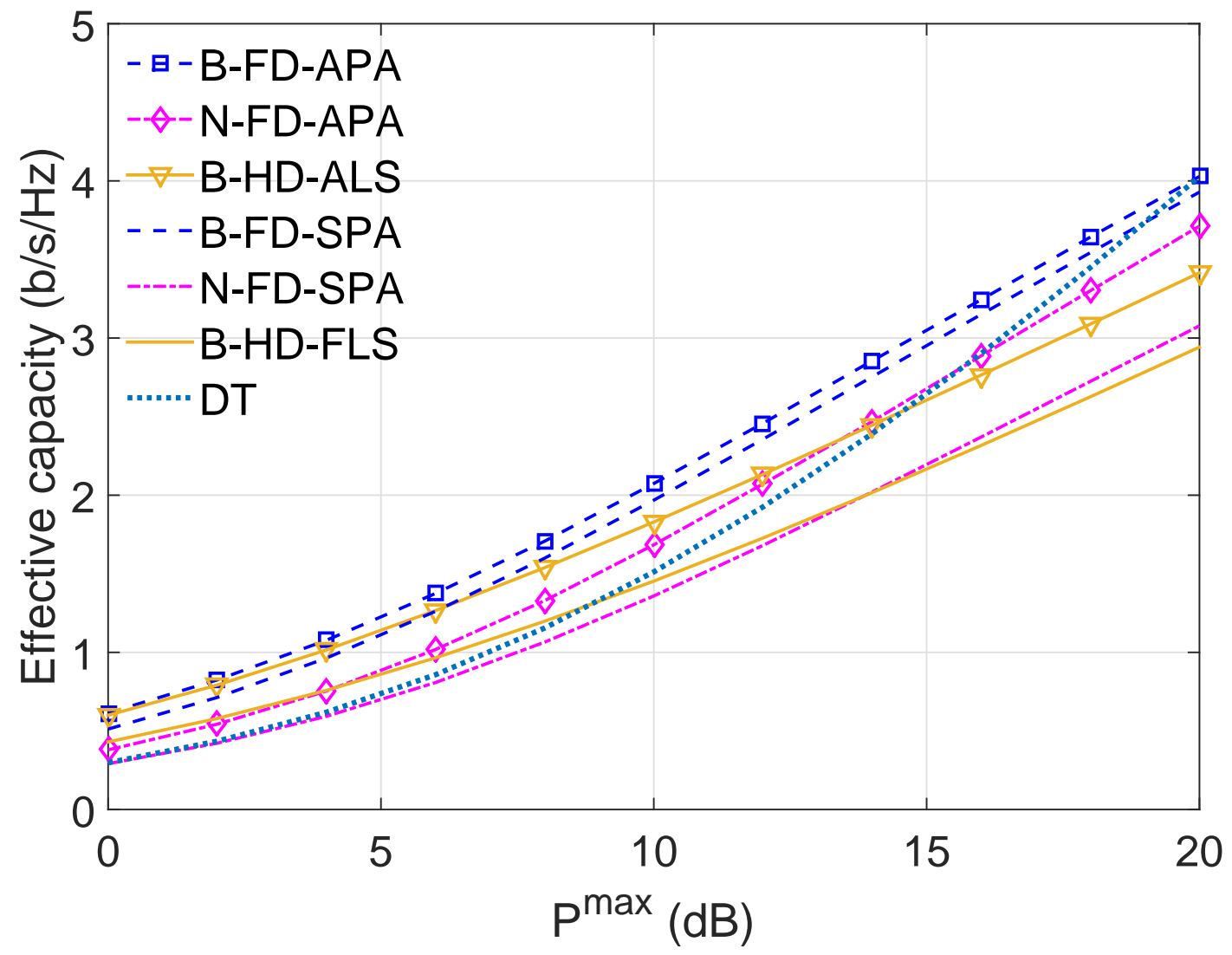

Fig. 6.5: Effective capacity versus $P^{\max }$ with $\zeta_{Q}=1$.

$P_{1}^{*}$, and $P_{2}^{*}$. We can derive the capacities of the different transmission schemes as:

$$
\begin{aligned}
\mu_{\mathrm{B}-\mathrm{FD}-\mathrm{SPA}} & =\mathbb{E}\left\{\log _{2}\left(1+h_{2}[t] P_{2}^{*}\right)\right\}, \\
\mu_{\mathrm{N}-\mathrm{FD}-\mathrm{SPA}} & =\mathbb{E}\left\{\log _{2}\left(1+\min \left\{h_{1}[t], h_{2}[t]\right\} P_{2}^{*}\right)\right\}, \\
\mu_{\mathrm{B}-\mathrm{HD}-\mathrm{FLS}} & =\frac{1}{2} \mathbb{E}\left\{\log _{2}\left(1+h_{2}[t] P^{\max }\right)\right\} \\
\mu_{\mathrm{DT}} & =\mathbb{E}\left\{\log _{2}\left(1+h_{\mathrm{SD}}[t] P^{\max }\right)\right\} \\
& =\mathbb{E}\left\{\log _{2}\left(1+h_{2}[t] P^{\max } / 4\right)\right\} .
\end{aligned}
$$

From the above expressions, we can see that $\mu_{\mathrm{B}-\mathrm{FD}-\mathrm{SPA}}>\mu_{\mathrm{N}-\mathrm{FD}-\mathrm{SPA}}$. Also, that $\mu_{\mathrm{B}-\mathrm{FD}-\mathrm{SPA}}>$ $\mu_{\mathrm{B}-\mathrm{HD}-\mathrm{FLS}}$ holds true when $P_{2}^{*}$ is larger than a threshold, or equivalently, $\beta$ needs to be 


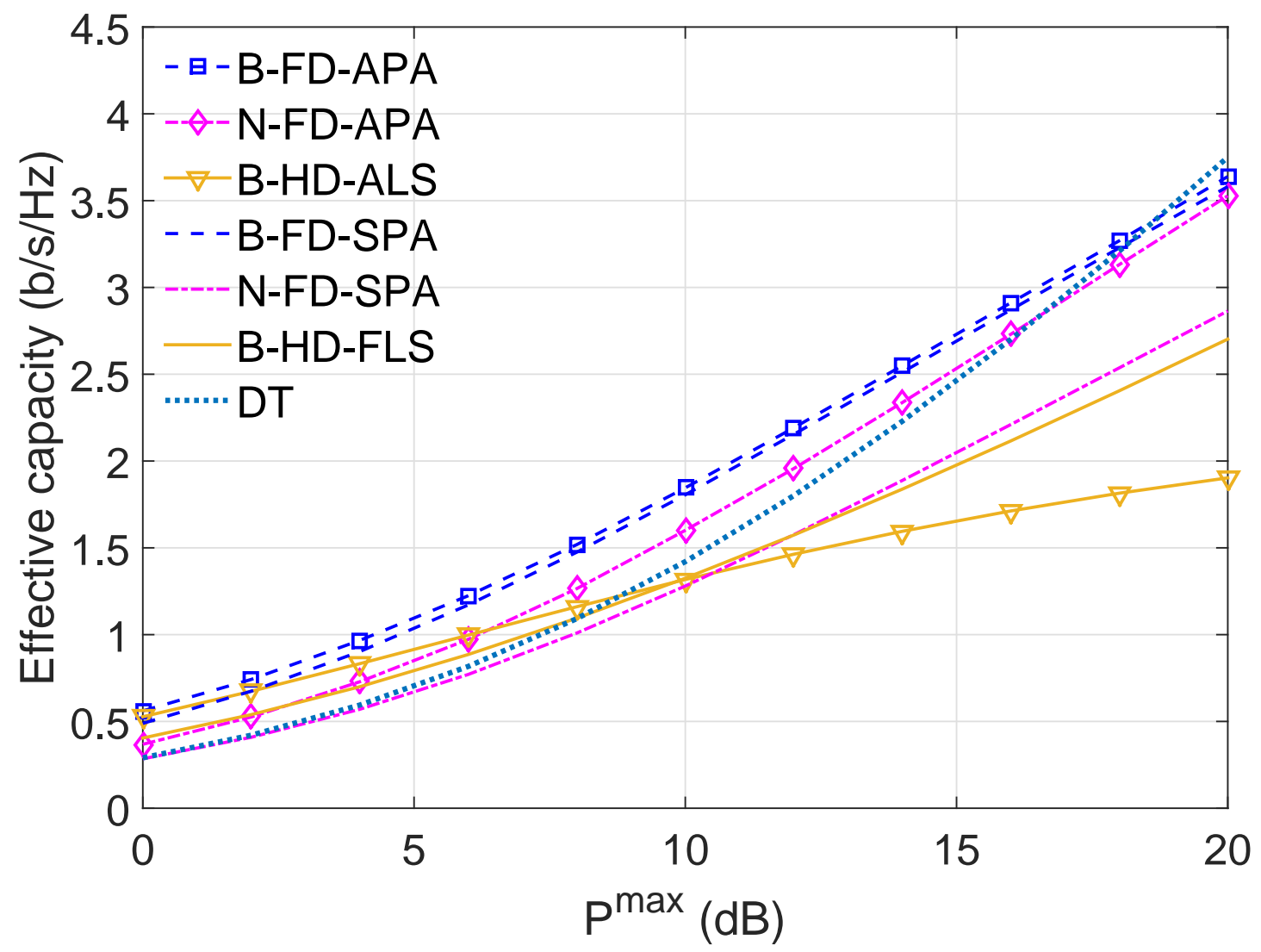

Fig. 6.6: Effective capacity versus $P^{\max }$ with $\zeta_{Q}=10^{-4}$.

smaller than a threshold, i.e., sufficiently good SI cancellation. Compared with DT, we can see that $\mu_{\mathrm{B}-\mathrm{FD}-\mathrm{SPA}}>\mu_{\mathrm{DT}}$ when $P_{2}^{*}>P^{\max } / 4$, i.e., $P_{2}^{*}$ is within $\sim 6 \mathrm{~dB}$ from $P^{\max }$. For instance, consider the case $\beta=.1$. For $P^{\max }=20 \mathrm{~dB}$, from Fig. 6.2, we have $P_{2}^{*}=13.64$ $\mathrm{dB}$, and hence, $\mu_{\mathrm{B}-\mathrm{FD}-\mathrm{SPA}}<\mu_{\mathrm{DT}}$ as shown in Fig. 6.5; For $P^{\max }=10 \mathrm{~dB}$, from Fig. 6.2, we have $P_{2}^{*}=6.11 \mathrm{~dB}$, and hence, $\mu_{\mathrm{B}-\mathrm{FD}-\mathrm{SPA}}>\mu_{\mathrm{DT}}$ as shown in Fig. 6.5. It implies that in order to have $\mu_{\mathrm{B}-\mathrm{FD}-\mathrm{SPA}}>\mu_{\mathrm{DT}}$, for a given $P^{\max }, \beta$ has to be smaller than a threshold; Or for a given $\beta, P^{\max }$ should be smaller than a threshold. Note that when $\beta=0$, i.e., zero residual SI, we have $P_{2}^{*}=P^{\text {max }} / 2$, implying B-FD-SPA is always more efficient than DT. These discussions are reflected in Figs. 6.3 and 6.5. 


\subsubsection{Effects of the Delay Constraint}

In this section, we fix $P^{\max }=20 \mathrm{~dB}$, and SI cancellation parameter $\beta=0, .1$ or 1 , which correspond to three different levels of SI cancellation. Note that the corresponding B-FDSPA and N-FD-SPA optimal solutions are shown in Fig. 6.2. We then vary $\zeta_{Q}$ and investigate its effects on the effective capacities of the relaying schemes.

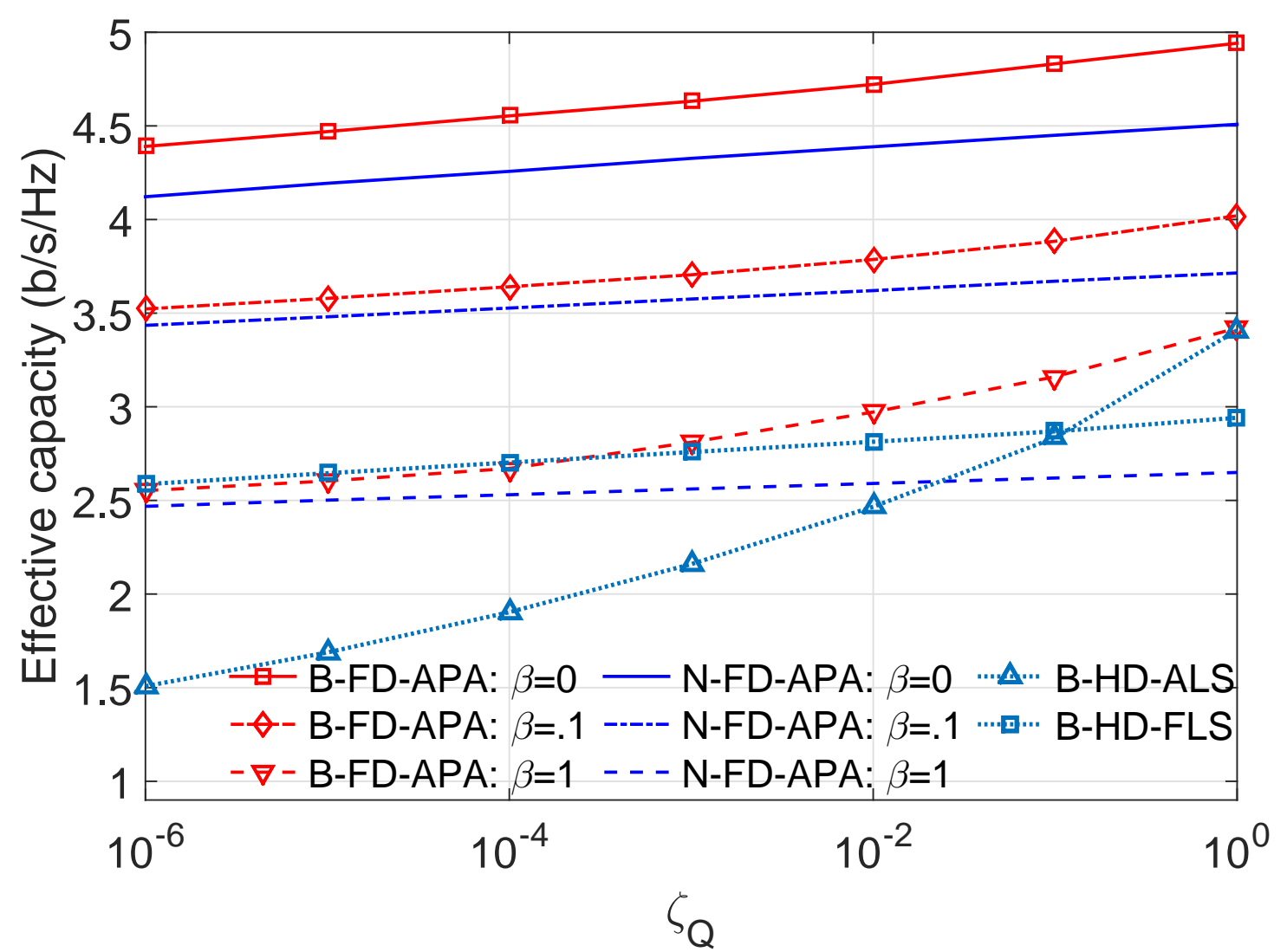

Fig. 6.7: Effective capacity versus $\zeta_{Q}$ with adaptive power allocation.

Consider the case with instantaneous CSIT. The capacities of B-FD-APA and N-FDAPA versus $\zeta_{Q}$ are shown in Fig. 6.7. We also plot the capacities of B-HD-ALS and B-HDFLS. As mentioned previously, under sufficiently stringent delay constraints, B-HD-FLS is more efficient than B-HD-ALS. First, we can see that B-FD-APA outperforms N-FD-APA as shown in the previous results, where the capacity gains are smaller under more stringent delay 
constraints (for a given $\beta$ ). This is because the data needs to be forwarded to the destination with very small delay, and hence, buffer-aided relaying may not be much useful. Second, BFD-APA outperforms H-FD relaying for sufficiently small $\beta$, and the gains are larger under more stringent delay constraints. For large $\beta=1$, B-FD-APA performs worse than B-HDFLS under sufficiently stringent delay constraints. As the delay constraint becomes looser, B-FD-APA provides capacity gains over B-HD-FLS as well as B-HD-ALS. Under very loose delay constraints, B-FD-APA approaches B-HD-ALS as mentioned in Section III.C and also illustrated in Fig. 6.3. We can see that, even for very bad SI cancellation, i.e., $\beta=1$, B-FD relaying can offer noticeable gains over B-FD relaying under neither stringent nor loose delay constraint region with instantaneous CSIT.

Next consider the case with statistical CSIT. The capacities of B-FD-SPA, N-FD-SPA, as well as B-HD-FLS versus $\zeta_{Q}$ are shown in Fig. 6.8. As seen, B-FD-SPA outperforms N-FDSPA, and the gains are larger as compared with the case of instantaneous CSIT. Moreover, B-FD-SPA outperforms B-FD-SPA for sufficiently small $\beta$. For large $\beta=1$, B-FD-SPA performs worse than B-FD-SPA, unlike the case with instantaneous CSIT, where B-FDAPA can even be more efficient than H-FD-ALS under sufficiently loose delay constraints.

\subsubsection{Summary of Key Results}

The presented numerical results have demonstrated the effects of SI cancellation, total power, delay constraint, as well as CSIT availability on the capacities of the transmission schemes. We summarize the following main observations.

- Compared with B-HD relaying, with instantaneous CSIT, B-FD relaying is more advantageous. For bad SI cancellation and under loose delay constraint, B-FD relaying approaches B-HD relaying. With only statistical CSIT, B-FD relaying is more beneficial for good SI cancellation.

- Compared with N-FD relaying, B-FD relaying achieves better performance under similar CSIT availability assumption.

- Compared with DT mode, B-FD-SPA relaying (for given $\beta$ ) is more spectrum-efficient except when the direct S-D link power becomes too strong.

- The capacity gains offered by adaptive power allocation over static power allocation 


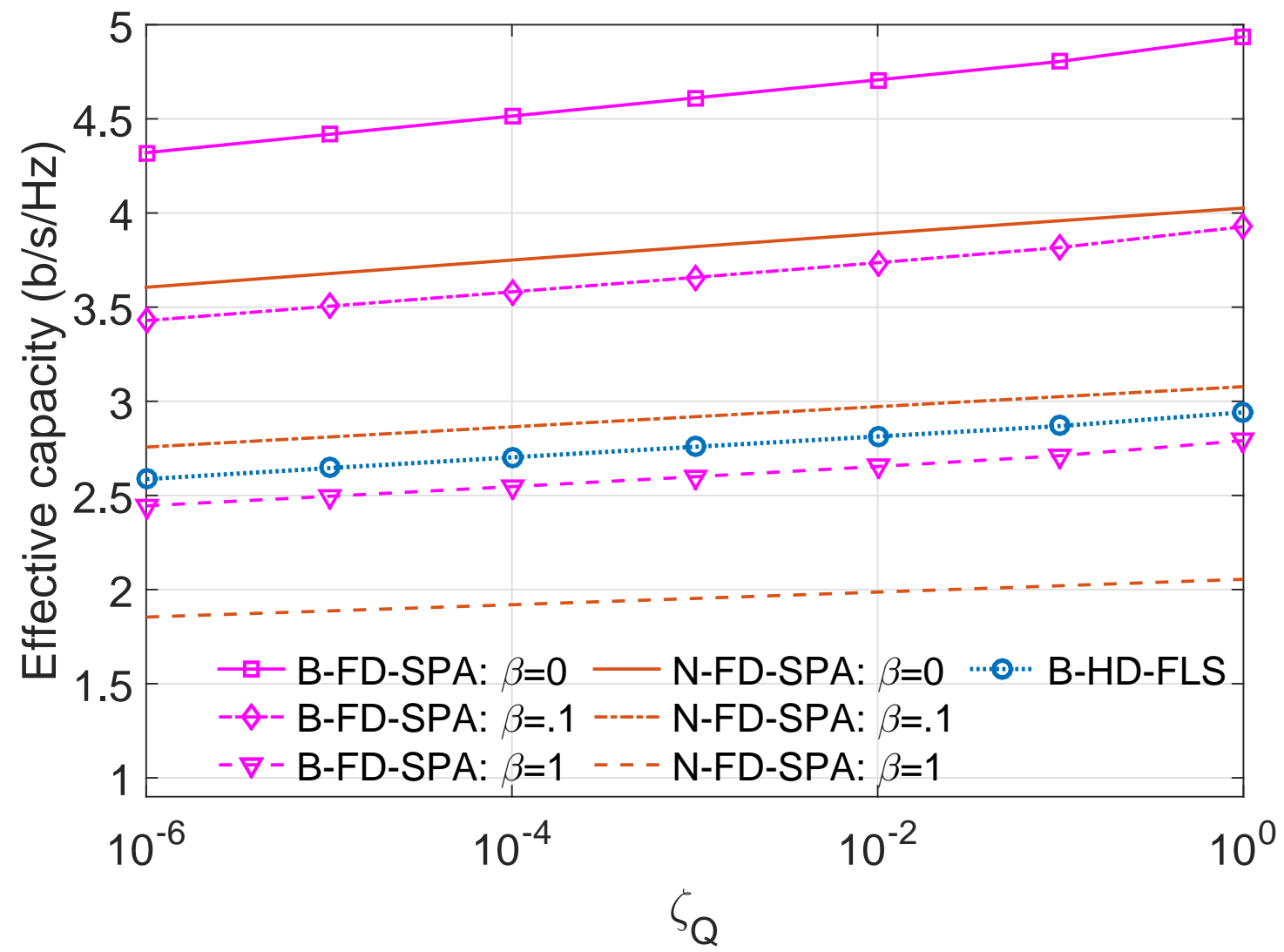

Fig. 6.8: Effective capacity versus $\zeta_{Q}$ with static power allocation.

in B-FD relaying decrease for better SI cancellation and under more stringent delay constraints.

\subsection{Chapter Summary}

In this chapter, we have studied the source and relay power allocation problems for a 3node buffer-aided full-duplex (B-FD) relaying network under the presence of imperfect selfinterference (SI) cancellation. The objective is to maximize the constant supportable source arrival rate under the delay-outage constraint in terms of maximum acceptable queue-lengthoutage probability. When the instantaneous channel state information is available at the transmitters (CSIT), we have investigated the adaptive power allocation problem (B-FD- 
APA) for each transmission frame. When only statistical CSIT is available, we have studied the static power allocation problem (B-FD-SPA). Assuming large delay regime, the optimal allocation solutions are derived using asymptotic delay analysis. Special cases on the delay constraint and residual SI are also investigated. Numerical studies are performed to show the performance of the proposed relaying schemes and other existing schemes under different settings. Our studies demonstrate the effectiveness of B-FD relaying to support delayconstrained communications. 


\section{Chapter 7}

\section{Conclusions and Future Works}

\subsection{Summary}

One main challenge for reliable wireless data transmission is the fading nature of wireless channels causing random fluctuations in the instantaneous channel conditions (and the corresponding transmission rates). As a result, the data arrivals at each transmitter might not be transmitted instantly, and need to be buffered for later transmissions. Such data buffering enables the transmitter to adapt its transmission to exploit temporal fading diversity, but at the same time introduces queuing delay. Providing delay QoS guarantees is a critical task in order to support delay-sensitive applications such as real-time multimedia streaming etc. Under such settings, radio resource allocation is an efficient means for throughput enhancement and delay QoS guarantees. Along this thesis, we investigate resource allocation schemes for practical point-to-point and relaying communications systems over fading channels to support delay-sensitive communications, where the nodes (source and relay) employ buffers to store the data arrivals. The main contributions and corresponding results are summarized as follows.

In Chapters 3, and 4, we consider a point-to-point communications system over a fading channel with data arriving at the source transmission buffer. More specifically, in Chapter 3 , the source is assumed to have maximum average power constraint. Also, average delay constraint is imposed for delay QoS guarantee. We studied the data admission control- power allocation (AC-PA) problem for throughput maximization. The problem is formulated as an infinite horizon constrained Markov decision process (MDP) problem, which can be solved using a novel approach based on the post-decision state-value function. Using the proposed 
approach, the structural properties of the optimal AC-PA solution, e.g., monotonicity and convexity with respect to the channel fading, data arrival, and queue length states are studied. We also develop online allocation algorithm when the statistical information about the random processes governing the system is unknown. By updating the post-decision statevalue function using realizations of the random processes, the developed algorithm requires less complexity and converge faster than traditional reinforcement learning algorithms [41]. It is demonstrated that the proposed approach achieves higher throughput than existing approaches under similar delay and power constraints, which do not keep track of the system dynamics and random variations in the data arrivals and channel conditions. Under large delay regime, the two approaches perform similarly since the effects of queue dynamics and random data arrivals become negligible.

In Chapter 4, an energy harvesting (EH) source is assumed, where a random amount of energy is harvested in every time slot and stored in battery during the course of data transmission. We have explored power allocation problems for such EH systems for source arrival rate maximization under either average delay or delay-outage constraints. For the latter case, by assuming large delay regime and employing asymptotic delay analysis, we have converted the original problem into an effective capacity (EC) maximization problem. We have formulated the power allocation problems as constrained MDP problems, which can be solved using post-decision state-value function approach. The formulations incorporate the randomness of the $\mathrm{EH}$ process and ensure causality constraint on the use of energy for data transmission. Properties of the optimal solutions are studied, which reveal valuable insights on how to optimally allocate the power according to the channel conditions and. It is illustrated that the proposed approaches achieve higher data rates than existing heuristic approaches, which do not take into account the random variations of energy arrivals. We have also demonstrated the different effects of the two delay constraint models on the system performance in terms of supported rates and delay performance.

In practice, it is not always possible for a source to communicate directly with the destination, for example, due to long distance, or severe shadowing. Under such scenarios, relaying communications is needed. Chapters 5 and 6 develop resource allocation schemes for 3-node buffer-aided relaying system under delay-outage constraint .

More specifically, in Chapter 5, we studied the optimal buffer-aided half-duplex (B-HD) relaying with adaptive link selection (B-HD-ALS) problem. In each frame, the relay can receive or transmit depending on the instantaneous channel state information (CSI). Both 
cases of fixed and adaptive power allocation are considered. By employing asymptotic delay analysis, we derive the optimal solution as a function of the instantaneous link conditions and delay constraint using Lagrangian approach and convex optimization. Solutions for various special cases of link conditions, and delay constraint are presented. Illustrative results show that B-HD-ALS outperforms B-HD with fixed relaying under sufficiently loose delay constraints. Also, adaptive power allocation provides significant gains over fixed power allocation as long as a certain delay can be tolerated. Also, B-HD-ALS is more beneficial than non-buffer relaying.

In Chapter 6, the relay is assumed to have buffer-aided full-duplex (B-FD) relaying capability with non-zero residual self-interference (SI). Toward this end, two source and relay transmit power allocation problems are investigated, which depend on the availability of the CSI at the transmitters (CSIT). With instantaneous CSIT, we have considered the B-FD with adaptive power allocation (B-FD-APA) problem. On the other hand, with statistical CSIT, the B-FD with static power allocation (B-FD-SPA) problem is considered. The optimal solutions are derived using Lagrangian approach for the constrained optimization problems. Solutions for special cases of delay constraint and residual SI are studied. It is demonstrated that B-FD-SPA relaying outperforms B-HD relaying under sufficiently small residual SI. However, B-FD-APA relaying always outperforms B-HD relaying since the former can dynamically switch between HD/FD relaying mode depending on the link conditions. When the residual SI becomes large, B-FD-APA approaches B-HD relaying. Moreover, B-FD relaying is more advantageous than non-buffer FD relaying under delay-outage constraint.

\subsection{Potential Future Works}

The research presented in this thesis has considered resource allocation for point-to-point or 3-node relaying communications networks, which are the two basic network segments forming more general wireless networks with multiple sources, multiple relays, and even multi-hop (more than two hops) communications. Hence, the proposed approaches and solutions in this thesis have the potential to be employed to tackle the challenging resource allocation designs for more general wireless systems, which are outlined in the following. 


\section{Relay selection, and resource allocation for general dual-hop networks}

In Chapters 5, and 6, we have considered resource allocation for 3-node buffer-aided relaying networks. However, in practice, multiple relays can be deployed to assist several sources over large communications area [125]- [127]. One potential future work is to study resource allocation schemes for such general dual-hop networks. Similar to our work in Chapters 5, and 6, buffer-aided relaying is considered. For delay QoS guarantees, we can impose delay outage constraint for each traffic flow originated from the sources.

For the considered networks, due to resource sharing among the sources, it is critical to determine which relay(s) to assist which source(s) to ensure performance satisfaction, i.e., relay selection problem. In addition to the relay selection problem, the resource allocation problem includes the link scheduling, rate, and power allocation for the sources and relays. Note that when a relay is transmitting, it must allocate the relay-destination link capacity to transmit data of its forwarded sources. In general, the optimal relay selection, and resource allocation solution has to be jointly considered, which has to take into account the delay and rate requirements of the sources. Such a problem remains unsolved.

\section{Cross-layer optimization for multi-hop wireless networks}

The ultimate goal is to study resource allocation schemes for general wireless multi-hop networks, where nodes at different locations transmit their own information as well as relay information of other nodes toward a common sink. In order to efficiently support real-time applications, for example, video streaming or surveillance applications, it is important to provision end-to-end delay QoS constraint for each data flow even when each flow requires multi-hop transmissions. In general, to achieve optimal network performance, a cross-layer design has to be considered, which includes joint routing, link scheduling, rate, and power allocation [128]. Unfortunately, such cross-layer design for multi-hop networks is challenging because it involves large number of variables to be optimized. Hence, efficient network designs with low complexity and delay constraint satisfaction are desirable.

Motivated by the above discussions, one potential future work is look for efficient crosslayer designs of multi-hop networks supporting delay-constrained communications. We observe that a multi-hop network is made up of multiple atomic network segments, each being a dual-hop or single-hop network. Then, by adapting the design results obtained for the single-hop and dual-hop networks, it is possible to devise sub-optimal but efficient designs 
for general multi-hop networks. 


\section{References}

[1] M. Agiwal, A. Roy, and N. Saxena, "Next Generation 5G Wireless Networks: A Comprehensive Survey," Commun. Surveys Tuts., vol. 18, no. 3, pp. 1617-1655, Feb. 2016.

[2] J. Andrews, S. Buzzi, W. Choi, S. Hanly, A. Lozano, A. Soong, and J. Zhang, "What Will 5G be?" IEEE J. Sel. Areas Commun., vol. 32, no. 6, pp. 1065-1082, June 2014.

[3] A. Goldsmith, Wireless Communications. Cambridge University Press, 2005.

[4] Z. Han, An Optimization Theoretical Framework for Resource Allocation over Wireless Networks. PhD thesis, University of Maryland, USA, 2003.

[5] M. Andrews, K. Kumaran, K. Ramanan, A. Stolyar, P. Whiting, and R. Vijayakumar, "Providing Quality of Service over a Shared Wireless Link," IEEE Commun. Mag., vol. 39, no. 2, pp. 150-154, Feb. 2001.

[6] J. Chen, Resource Allocation for Delay Constrained Wireless Communications. PhD thesis, University College London, UK, 2010.

[7] X. Zhang, W. Cheng, and H. Zhang, "Heterogeneous Statistical QoS Provisioning Over 5G Mobile Wireless Networks," IEEE Network Magazine, vol. 28, no. 6, pp. 46-53, Nov./Dec. 2014.

[8] 3rd Generation Partnership Project, Universal Mobile Telecommunications System (UMTS); Quality of Service Concept and Architecture. (3GPP TS 23.107 version 5.4.0 Release 5).

[9] B. Collins, and R. Cruz, "Transmission Policies for Time Varying Channels with Average Delay Constraints," in Proc. 1999 Allerton Conf. on Commun., Control, and Computing, Urbana, IL, USA.

[10] X. Zhang, J. Tang, H.-H. Chen, S. Ci, and M. Guizani, "Cross-layer-based Modeling for Quality of Service Guarantees in Mobile Wireless Networks," IEEE Commun. Mag., vol. 44, no. 1, pp. 100-106, Jan. 2006. 
[11] S. Meninger, J. O. Mur-Miranda, R. Amirtharajah, A. Chandrakasan, and J. H. Lang, "Vibration-to-Eletric Energy Conversion," IEEE Trans. on VLSI, vol. 9, no.1, pp. 6476, Feb. 2001.

[12] V. Raghunathan, A. Kansal, J. Hsu, J. Friedman, and M. B. Srivastava, "Design Considerations for Solar Energy Harvesting Wireless Embedded Systems," in Proc. 2005 IEEE IPSN, Los Angeles, CA, USA.

[13] S. Chalasani, and J. M. Conrad, "A Survey of Energy Harvesting Sources for Embedded Systems," in Proc. 2008 IEEE Southeastcon, Huntsville, AL, USA.

[14] M. Gorlatova, P. Kinget, I. Kymissis, D. Rubenstein, X. Wang, and G. Zussman, "Challenge: Ultra-low-power Energy-harvesting Active Networked Tags (EnHANTs), " in Proc. 2009 ACM Mobicom, Beijing, China.

[15] H. Li, C. Huang, F. Alsaadi, A. M. Dobaie, and S. Cui, "Energy Harvesting based Green Heterogeneous Wireless Access for 5G," in 5G Mobile Communications, Ed., Springer, 2016, pp. 475-502.

[16] D. Mishra, and S. De, "Energy Harvesting and Sustainable M2M Communication in 5G Mobile Technologies," in Internet of Things (IoT) in 5G Mobile Technologies, Ed., Springer, 2016, pp 99-125.

[17] H. Shafieirad, R. S. Adve, and S. ShahbazPanahi, "Large Scale Energy Harvesting Sensor Networks with Applications in Smart Cities," in Smart City 360, Ed., Springer, 2016, pp. 215-226.

[18] T. R. Halford, and K. M. Chugg, "Barrage Relay Networks," in Proc. 2010 IEEE ITA, San Diego, CA, USA.

[19] D. Gunduz, K. Stamatiou, N. Michelusi, and M. Zorzi, "Designing Intelligent Energy Harvesting Communication Systems," IEEE Commun. Mag., vol. 52, no. 1, pp. 210216, Jan. 2014.

[20] I. Ahmeda, M. M. Butta, C. Psomasb, A. Mohamed, I. Krikidisb, and M. Guizania, "Survey on Energy Harvesting Wireless Communications: Challenges and Opportunities for Radio Resource Allocation," Computer Networks, vol. 88, no. 9, pp. 234-248, Sept. 2015.

[21] S. Ulukus, A. Yener, E. Erkip, O. Simeone, M. Zorzi, P. Grover, and K. Huang. "Energy Harvesting Wireless Communications: A Review of Recent Advances," IEEE J. Sel. Areas Commun., vol. 33, no. 5, pp. 360-381, Mar. 2015. 
[22] Y. Cui, V. Lau, R. Wang, and H. Huang, "A Survey on Delay-aware Resource Control for Wireless Systems - Large Derivation Theory, Stochastic Lyapunov Drift and Distributed Stochastic Learning," IEEE Trans. Infor. Theory, vol. 58, no. 3, pp. $1677-$ 1701, March 2012.

[23] R. Berry, and R. Gallager, "Communication over Fading Channels with Delay Constraints," IEEE Trans. Infor. Theory, vol. 48, no. 5, pp. 1135-1149, May 2002.

[24] D. S. W. Hui, V. K. N. Lau, and H. L. Wong, "Cross-layer Design for OFDMA Wireless Systems with Heterogeneous Delay Requirements," IEEE Trans. Wireless Commun., vol. 6, no. 8, pp. 2872-2880, Aug. 2007.

[25] M. J. Neely, "Energy Optimal Control for Time Varying Wireless Networks," IEEE Trans. Infor. Theory, vol. 52, no. 7, pp. 2915-2934, July 2006.

[26] N. Salodkar, A. Bhorkar, A. Karandikar, and V. S. Borkar, "On-Line Learning Algorithm for Energy Efficient Delay Constrained Scheduling over Fading Channel," IEEE J. Sel. Areas Commun., vol. 26, no. 4, pp. 732-742, May 2008.

[27] F. Fu, and M. van der Schaar, "Structure-Aware Stochastic Control for Transmission Scheduling," IEEE Trans. Veh. Tech., vol. 61, no. 9, pp. 3931-3945, Nov. 2012.

[28] A. Abdrabou and W. Zhuang, "Stochastic Delay Guarantees and Statistical Call Admission Control for IEEE 802.11 Single-hop Ad hoc Networks," IEEE Trans. Wireless Commun., vol. 7, no. 10, pp. 3972-3981, Oct. 2008.

[29] M. Zafer, and E. Modiano, "Minimum Energy Transmission over a Wireless Channel with Deadline and Power Constraints," IEEE Trans. Auto. Control, vol. 54, no. 12, pp. 2841-2852, Dec. 2009.

[30] W. Chen, M. J. Neely, and U. Mitra, "Energy-efficient Transmissions with Individual Packet Delay Constraints," IEEE Trans. Inf. Theory, vol. 54, no. 5, pp. 2090-2109, May 2008.

[31] V. Hanly, and D. Tse, "Multiaccess Fading Channels. Part II: Delay-limited Capacities,"IEEE Trans. Inf. Theory, vol. 44, no. 7, pp. 2816óÀẼ2831, Nov. 1998.

[32] D. Wu, and R. Negi, "Effective Capacity: A Wireless Link Model for Support of Quality of Service," IEEE Trans. Wireless Commun., vol. 2, no. 4, pp. 630-643, Jul. 2003.

[33] D. Wu, Providing Quality-of-Service Guarantees in Wireless Networks. PhD thesis, Carnegie Mellon University, USA, 2003.

[34] C.-S Chang, "Stability, Queue Length, and Delay of Deterministic and Stochastic Queuing Networks," IEEE Trans. Auto. Control, vol. 39, no. 5, pp. 913-931, May 1994. 
[35] X. Zhang, and Q. Du, "Cross-Layer Modeling for QoS-Driven Multimedia Multicast/Broadcast Over Fading Channels in Mobile Wireless Networks," IEEE Commun. Mag., vol. 45, no. 8, pp. 62-70, Aug. 2007.

[36] E. Altman, Constrained Markov Decision Processes: Stochastic Modeling. London, UK.: Chapman \& Hall CRC, 1999.

[37] P. Blasco, D. Gunduz, and M. Dohler, "A Learning Theoretic Approach to Energy Harvesting Communication System Optimization," IEEE Trans. Wireless Commun., vol. 12, no. 4, pp. 1872-1882, Apr. 2013.

[38] O. Ozel, K. Tutuncuoglu, J. Yang, S. Ulukus, and A. Yener, "Transmission with Energy Harvesting Nodes in Fading Wireless Channels: Optimal Policies," IEEE J. Sel. Areas Commun., vol. 29, no. 8, pp. 1732-1743, Sept. 2011.

[39] C. K. Ho, and R. Zhang, "Optimal Energy Allocation for Wireless Communications with Energy Harvesting Constraints," IEEE Trans. Signal Process., vol. 60, no. 9, pp. 4808-4818, Sept. 2012.

[40] D. P. Bertsekas. Dynamic Programming and Optimal Control Vol. 1. Belmont, MA: Athens Scientific, 1995.

[41] R. S. Sutton, and A. G. Barto, Reinforcement Learning: An Introduction. The MIT Press, 1998.

[42] V. S. Borkar, Stochastic Approximation: A Dynamical Systems Viewpoint. Cambridge University Press, 2008.

[43] B. Xia, Y. Fan, J. Thompson, and H. V. Poor, "Buffering in a Three-node Relay Network," IEEE Trans. Wireless Commun., vol. 7, no. 11, pp. 4492-4496, Nov. 2008.

[44] I. Krikidis, T. Charalambous, and J. Thompson, "Buffer-aided Relay Selection for Cooperative Diversity Systems without Delay Constraints," IEEE Trans. Wireless Commun., vol. 11, no. 5, pp. 1957-1967, 2012.

[45] N. Zlatanov, A. Ikhlef, T. Islam, and R. Schober, "Buffer-aided Cooperative Communications: Opportunities and Challenges," IEEE Commun. Magazine, vol. 52, no. 4, pp. 146-153, April 2014.

[46] N. Zlatanov, R. Schober, and P. Popovski, "Buffer-aided Relaying with Adaptive Link Selection," IEEE J. Sel. Areas Commun., vol. 31, no. 8, pp. 1530-1542, Aug. 2013.

[47] N. Zlatanov, and R. Schober, "Buffer-aided Relaying With Adaptive Link Selection Fixed and Mixed Rate Transmission," IEEE Trans. on Inform. Theory, vol. 59, no. 5, pp. 2816-2840, Jan. 2013. 
[48] M. Jain, J. I. Choi, T. M. Kim, D. Bharadia, S. Seth, K. Srinivasan, P. Levis, S. Katti, and P. Sinha, "Practical, Real-time, Full-Duplexing Wireless," in Proc. 2011 ACM Mobicom, Las Vegas, NV, USA.

[49] M. Duarte, C. Dick, and A. Sabharwal, "Experiment-driven Characterization of Fullduplex Wireless Systems," IEEE Trans. Wireless Commun., vol. 11, no. 12, pp. 42964307, Dec. 2012.

[50] D. Bharadia, E. McMilin, and S. Katti, "Full-Duplex Radios," in SIGCOMM Comput. Commun. Rev., vol. 43, no. 4, pp. 375-386, Aug. 2013.

[51] T. Riihonen, S. Werner, and R. Wichman, "Comparison of Full-duplex and Half-duplex Modes with a Fixed Amplify-and-Forward Relay," in Proc. 2009 IEEE WCNC, Budapest, Hungary.

[52] T. Riihonen, S. Werner, and R. Wichman, "Hybrid Full-duplex/Half-duplex Relaying with Transmit Power Adaptation," IEEE Trans. Wireless Commun., vol. 10, no. 9, pp. 3074-3085, Sept. 2011.

[53] H. Q. Ngo, H. A. Suraweera, M. Matthaiou, and E. G. Larsson, "Multipair Full-duplex Relaying with Massive Arrays and Linear Processing," IEEE J. Sel. Areas Commun., vol. 32, no. 9, pp. 1721-1737, June 2014.

[54] L. J. Rodriguez, N. H. Tran, and T. Le-Ngoc, "Optimal Power Allocation and Capacity of Full-Duplex AF Relaying under Residual Self-Interference," IEEE Wireless Commun. Letters, vol. 3, no. 2, pp. 233-236, Apr. 2014.

[55] D. Rajan, A. Sabharwal, and B. Aazhang, "Delay Bounded Packet Scheduling of Bursty Traffic over Wireless Channels," IEEE Trans. Infor. Theory, vol. 50, no. 1, pp. 125-144, Jan. 2004.

[56] H.Wang, and N. B. Mandayam, "A Simple Packet-transmission Scheme for Wireless Data over Fading Channels," IEEE Trans. Commun., vol. 52, no. 7, pp. 1055-1059, July 2004.

[57] M. Agarwal, V. Borkar, and A. Karandikar, "Structural Properties of Optimal Transmission Policies over a Randomly Varying Channel," IEEE Trans. Autom. Control, vol. 53, no. 6, pp. 1476-1491, July 2008.

[58] D. Djonin, and V. Krishnamurthy, "Transmission Control in Fading Channels - A Constrained Markov Decision Process Formulation with Monotone Randomized Policies," IEEE Trans. Signal Process., vol. 55, no. 10, pp. 5069-5083, Oct. 2007. 
[59] D. Djonin, and V. Krishnamurthy, "Q-Learning Algorithms for Constrained Markov Decision Processes with Randomized Monotone Policies: Applications to MIMO Transmission Control," IEEE Trans. Signal Process., vol. 55, no. 5, pp. 2170-2181, May 2007.

[60] C.-S. Chang, and T. Zajic, "Effective Bandwidths of Departure Processes from Queues with Time Varying Capacities," in Proc. 1995 IEEE INFOCOMM, Boston, MA, USA.

[61] J. Tang and X. Zhang, "QoS-driven Power and Rate Adaptation over Wireless Links," IEEE Trans. Wireless Commun., vol. 6, no. 8, pp. 3058-3068, Aug. 2007.

[62] J. Tang, and X. Zhang, "Quality-of-Service Driven Power and Rate Adaptation for Multichannel Communications Over Wireless Links," IEEE Trans. Wireless Commun., vol. 6, no. 12, pp. 4349-4360, Dec. 2007.

[63] X. Zhang, and J. Tang, "Power-delay Tradeoff over Wireless Networks," IEEE Trans. Commun., vol. 61, no. 9, pp. 3673-3684, Sept. 2013.

[64] A. Helmy, L. Musavian, and T. Le-Ngoc, "Energy-efficient Power Adaptation over a Frequency-selective Fading Channel with Delay and Power Constraints," IEEE Trans. Wireless Commun., vol. 12, no. 9, pp. 4529-4541, Sept. 2013.

[65] M. C. Gursoy, "MIMO Wireless Communications under Statistical Queueing Constraints," IEEE Trans. Infor. Theory, vol. 57, no. 9, pp. 5897-5917, Sept. 2011.

[66] D. Qiao, M. C. Gursoy, and S. Velipasalar, "Transmission Strategies in Multiple Access Fading Channels with Statistical QoS Constraints," IEEE Trans. Infor. Theory, vol. 58, no. 3, pp. 1578-1593, Mar. 2012.

[67] D. Wu, and R. Negi, "Downlink Scheduling in a Cellular Network for Quality-of-service Assurance," IEEE Trans. Veh. Technol., vol. 53, no. 5, pp. 1547-1557, Sep. 2004.

[68] D. Wu, and R. Negi, "Utilizing Multiuser Diversity for Efficient Support of Qualityof-service over a Fading Channel," IEEE Trans. Veh. Technol., vol. 54, no. 3, pp. 1198-1206, May 2005.

[69] K. T. Phan, and T. Le-Ngoc, "Dynamic Scheduling with Statistical Delay Guarantees and Traffic Dropping," in Proc. 2013 IEEE VTC-Spring, Dresden, Germany.

[70] K. T. Phan, Tho Le-Ngoc, "Online QoS-based Dynamic Scheduling in Multi-channel Wireless Networks," in Proc. 2013 IEEE WCNC, Shanghai, China.

[71] W. Cheng, X. Zhang, and H. Zhang, "Joint Spectrum and Power Efficiencies Optimization for Statistical QoS Provisionings Over SISO/MIMO Wireless Networks," IEEE J. Sel. Areas Commun., vol. 31, no. 5, pp. 903-915, May 2013. 
[72] L. Huang, and M. Neely, "Utility Optimal Scheduling in Energy-harvesting Networks," IEEE/ACM Trans. Net., vol. 21, no. 4, pp. 1117-1130, Aug. 2013.

[73] M. Gatzianas, L. Georgiadis, and L. Tassiulas, "Control of Wireless Networks with Rechargeable Batteries," IEEE Trans. Wireless Commun., vol. 9, no. 2, pp. 581-593, Feb. 2010.

[74] J. Gong, S. Zhang, X. Wang, S. Zhou, and Z. Niu, "Supporting Quality of Service in Energy Harvesting Wireless Links: The Effective Capacity Analysis," in Proc. 2014 IEEE ICC Workshops, Sydney, NSW, Australia.

[75] S. W. Peters, A. Y. Panah, K. T. Truong, and R. W. Heath, "Relaying Architectures for 3GPP LTE-Advanced," EURASIP Journal on Advances in Signal Processing, vol. 2009, Article ID 618787.

[76] M. O. Hasna and M.-S. Alouini, "End-to-End Performance of Transmission System with Relays over Rayleigh-fading Channels," IEEE Trans. Wireless Commun., vol. 2, no. 6, pp. 1126-1131, Nov. 2003.

[77] B. Wang, J. Zhang, and A. Host-Madsen, "On the Capacity of MIMO Relay Channels," IEEE Trans. Inf. Theory, vol. 51, no. 1, pp. 29-43, Jan. 2005.

[78] A. Ikhlef, D. S. Michalopoulos, and R. Schober, "Max-max Relay Selection for Relays with Buffers," IEEE Trans. Wireless Commun., vol. 11, no. 3,pp. 1124-1135, Mar. 2012 .

[79] D. Qiao, M. C. Gursoy, and S. Velipasalar, "Effective Capacity of Two-Hop Wireless Communication Systems," IEEE Trans. Infor. Theory, vol. 59, no. 2, pp. 873-885, Feb. 2013.

[80] K. T. Phan and T. Le-Ngoc, "Effective Capacities of Dual-Hop Networks with Relay Selection," in Proc. 2014 IEEE WCNC, Istanbul, Turkey.

[81] B. Zhou, Y. Liu, and M. Tao, "Adaptive Scheduling for OFDM Bidirectional Transmission with A Buffered Relay," in Proc. 2013 IEEE WCNC, Shanghai, China.

[82] V. Jamali, N. Zlatanov, and R. Schober, "Bidirectional Buffer-aided Relay Networks with Fixed Rate Transmission - Part I: Delay-Unconstrained Case," IEEE Trans. Wireless Commun., vol. 14, no. 3, pp. 1323-1338, Oct. 2014.

[83] V. Jamali, N. Zlatanov, and R. Schober, "Bidirectional Buffer-aided Relay Networks with Fixed Rate Transmission - Part II: Delay-Constrained Case," IEEE Trans. Wireless Commun., vol. 14, no. 3, pp. 1339-1355, Oct. 2014. 
[84] H. Shoukry, N. Zlatanov, V. Jamali, and R. Schober, "Achievable Rates for the Fading Three-Hop Half-Duplex Relay Network using Buffer-Aided Relaying," in Proc. 2014 IEEE GLOBECOM, Austin, TX, USA.

[85] G. Liu, F. R. Yu, H. Ji, V. Leung, and X. Li, "In-Band Full-Duplex Relaying: A Survey, Research Issues and Challenges," IEEE Commun. Surveys \&5 Tutorials, vol 17, no. 2, pp. 500-524, Jan. 2015.

[86] A. Del Coso, and C. Ibars, "Achievable Rates for the AWGN Channel with Multiple Parallel Relays," IEEE Trans. Wireless Commun., vol. 8, no. 5, pp. 2524-2534, May 2009.

[87] R. Nikjah, and N. Beaulieu, "Achievable Rates and Fairness in Rateless Coded Decodeand-Forward Half-duplex and Full-duplex Opportunistic Relaying," in Proc. 2008 IEEE $I C C$, Beijjing, China.

[88] N. Zlatanov, and R. Schober, "Buffer-aided Half-duplex Relaying can Outperform Ideal Full-duplex Relaying," IEEE Commun. Lett., vol. 17, no. 3, pp. 479-482, Mar. 2013.

[89] N. Zlatanov, D. Hranilovic, and J. S. Evans, "Buffer-Aided Relaying Improves Throughput of Full-Duplex Relay Networks with Fixed-Rate Transmissions," IEEE Commun. Letters, vol. , no. 99, pp. , Sept. 2016.

[90] B. Rankov, and A. Wittneben, "Achievable Rate Regions for the Two-Way Relay Channel," in Proc. 2006 IEEE ISIT, Seattle, WA, USA.

[91] R. Vaze, and R. W. Heath, "On the Capacity and Diversity-Multiplexing Tradeoff of the Two-Way Relay Channel," IEEE Trans. Inf. Theory, vol. 57, no. 7, pp. 4219-4234, July 2011.

[92] T. Riihonen, S. Werner, and R. Wichman, "Mitigation of Loopback Self-interference in Full-duplex MIMO Relays," IEEE Trans. Signal Process., vol. 59, no. 12, pp. 59835993, Dec. 2011.

[93] D. Ng, E. Lo, and R. Schober, "Dynamic Resource Allocation in MIMO-OFDMA Systems with Full-duplex and Hybrid Relaying," IEEE Trans. Commun., vol. 60, no. 5, pp. 1291-1304, May 2012.

[94] B. Day, A. Margetts, D. Bliss, and P. Schniter, "Full-duplex MIMO Relaying: Achievable Rates under Limited Dynamic Range," IEEE J. Sel. Areas Commun., vol. 30, no. 8, pp. 1541-1553, Sep. 2012

[95] N. Shende, O. Gurbuz, and E. Erkip, "Half-Duplex or Full-Duplex Relaying: A Capacity Analysis under Self-Interference," in Proc. 2013 IEEE CISS, Baltimore, MD, USA. 
[96] Q. Wang, Y. Dong, X. Xu, and X. Tao, "Outage Probability of Full-Duplex AF Relaying With Processing Delay and Residual Self-Interference," IEEE Commun. Lett., vol. 19, no. 5, pp. 783-786, May 2015.

[97] K. Yang, H. Cui, L. Song, and Y. Li, "Efficient Full-Duplex Relaying with Joint Antenna-Relay Selection and Self-Interference Suppression," IEEE Trans. Wireless Commun., vol. 14, no. 7, pp. 4052-4062, Jul. 2015.

[98] K. T. Phan, T. Le-Ngoc, M. van der Schaar, and F. Fu, "Joint Scheduling- Traffic Admission Control: Structural Results and Online Learning Algorithm," in Proc. 2013 IEEE ICC, Budapest, Hungary.

[99] K. T. Phan, T. Le-Ngoc, M. van der Schaar, and F. Fu, "Optimal Scheduling over Time-varying Channels with Traffic Admission Control: Structural Results and Online Learning Algorithms," IEEE Trans. Wireless Commun., vol. 12, no. 9, pp. 4434-4444, Sept. 2013.

[100] R. Amir, "Supermodularity and Complementarity in Economics: An Elementary Survey," Southern Economic Journal, vol. 71, no. 3, pp. 636-660, 2005.

[101] X. Wang, and G. B. Giannakis, "Resource Allocation for Wireless Multiuser OFDM Networks," IEEE Trans. Inf. Theory, vol. 57, no. 7, pp. 4359-4372, July 2011.

[102] I. Ahmed, K. T. Phan, and T. Le-Ngoc, "Optimal Stochastic Power Control for Energy Harvesting Systems with Statistical Delay Constraint," in Proc. 2015 IEEE GLOBECOM, San Diego, CA, USA.

[103] I. Ahmed, K. T. Phan, and T. Le-Ngoc, "Optimal Stochastic Power Control for Energy Harvesting Systems with Delay Constraints," IEEE J. Sel. Areas Commun. (JSAC), Series on Green Communications and Networking. To appear in 2016.

[104] M. Jayalakshmi, and K. Balasubramanian, "Simple Capacitors to Supercapacitors An Overview," Int. J. Electrochem. Sci., vol. 3, no. 11, pp. 1196-1217, Oct. 2008.

[105] N. Zlatanov, Z. Hadzi-Velkov, and R. Schober, "Asymptotically Optimal Power Allocation for Point-to-Point Energy Harvesting Communication Systems," in Proc. 2013 IEEE GLOBECOM, Atlanta, GA, USA.

[106] S. Boyd, and L. Vandenberghe. Convex Optimization. Cambridge University Press, 2004.

[107] K. T. Phan, and T. Le-Ngoc, "Adaptive Link Selection in Buffer-Aided Relaying with Statistical QoS Constraints," in Proc. 2015 IEEE ICC, London, UK. 
[108] K. T. Phan, T. Le-Ngoc, and L. Le, "Optimal Resource Allocation for Buffer-Aided Relaying with Statistical QoS Constraint," IEEE Trans. Commun., vol. 64, no. 3, pp. 959-972, Mar. 2016.

[109] J. Tang, and X. Zhang, "Cross-layer Resource Allocation over Wireless Relay Networks for Quality of Service Provisioning," IEEE J. Sel. Areas Commun., vol. 25, no. 4, pp. 645-656, May 2007.

[110] Q. Du, and C. Zhang, "Queuing Analyses and Statistically Bounded Delay Control for Two-hop Green Wireless Relay Transmissions," Concurrency and Computation: Practice and Experience, vol. 25, no. 9, pp. 1050-1063, June 2013.

[111] F. Chapeau-Blondeau, A. Monir, "Numerical Evaluation of the Lambert W Function and Application to Generation of Generalized Gaussian Noise with Exponent 1/2," IEEE Trans. Signal Process., vol. 50, no. 9, pp. 2160-2165, Sept. 2002.

[112] M. L. Fisher, "The Lagrangian Relaxation Method for Solving Integer Programming Problems," Management Science, vol. 50, no. 12, pp. 1861-1871, Dec. 2004.

[113] A. Bhorkar, A. Karandikar, and V. S. Borkar, "Power Optimal Opportunistic Scheduling," in Proc. 2006 IEEE GLOBECOM, San Francisco, CA, USA.

[114] C. T. K. Ng, and A. J. Goldsmith, "The Impact of CSI and Power Allocation on Relay Channel Capacity and Cooperation Strategies," IEEE Trans. on Wireless Commun., vol. 7, no. 12, pp. 5380-5389, Dec. 2008.

[115] N. Hassan, C. Yuen, S. Saeed, and Z. Zhang, "Power Control for Sum-Rate Maximization on Interference Channels Under Sum Power Constraint," IEEE Trans. Veh. Technol., vol. 64, no. 2, pp. 593-609, Feb. 2015.

[116] I. Hammerstrom, and A. Wittneben, "Joint Power Allocation for Non-regenerative MIMO-OFDMA Relay Links," in Proc. 2006 IEEE ICASSP, Toulouse, France.

[117] R. Ruby, V. Leung, and D. G. Michelson, "Centralized and Game Theoretical Solutions of Joint Source and Relay Power Allocation for AF Relay based Network," IEEE Trans. Commun., vol. 63, no. 8, pp. 2848-2863, June 2015.

[118] K. T. Phan, and T. Le-Ngoc, "Buffer-Aided Full-Duplex Relaying with Residual SelfInterference and Statistical Delay Provisioning," in Proc. 2015 IEEE PIMRC, Hong Kong, China.

[119] K. T. Phan, and T. Le-Ngoc, "Power Allocation for Buffer-Aided Full-Duplex Relaying with Imperfect Self-Interference (SI) Cancellation and Statistical Delay Constraint," IEEE Access, vol. 4, pp. 3961-3974, 2016. 
[120] D. Qiao, "The Impact of Statistical Delay Constraints on the Energy Efficiency in Fading Channels," IEEE Trans. Commun., vol. 15, no. 2, pp. 994-1007, Sept. 2015.

[121] M. Luby, "LT Codes," in Proc. 2002 IEEE Symp. Found. Comp. Sci., Vancouver, BC, Canada.

[122] A. Shokrollahi, "Raptor Codes," IEEE Trans. Infor. Theory, vol. 52, no. 6, pp. 25512567, June 2006.

[123] J. Casture, and Y. Mao, "Rateless Coding over Fading Channels," IEEE Commun. Lett., vol. 10, no. 1, pp. 46-48, Jan. 2006.

[124] C. W. Tan, M. Chiang, and R. Srikant, "Maximizing Sum Rate and Minimizing MSE on Multiuser Downlink: Optimality, Fast Algorithms, and Equivalence via Max-min SIR," IEEE Trans. Signal Process., vol. 59, no. 12, pp. 6127-6143, Dec. 2011.

[125] E. Beres, and R. Adve, "Selection Cooperation in Multi-source Cooperative Networks," IEEE Trans. Wireless Commun., vol. 7, no. 1, pp. 118-127, Jan. 2008.

[126] H. Ding, J. Ge, D. B. da Costa, and Z. Jiang, "A New Efficient Low-Complexity Scheme for Multi-Source Multi-Relay Cooperative Networks," IEEE Trans. Veh. Technol., vol. 60, no. 2, pp. 716-722, Sept. 2012.

[127] S. Atapattu, Y. Jing, H. Jiang, and C. Tellambura, "Relay Selection and Performance Analysis in Multiple-user Networks," IEEE J. Sel. Areas Commun., vol. 31, no. 8, pp. 1-13, Aug. 2013.

[128] E. Setton, T. Yoo, X. Zhu, A. Goldsmith, and B. Girod, "Cross-layer Design of Ad hoc Networks for Real-time Video Streaming," IEEE Wireless Commun. Mag., vol. 12, no. 4, pp. 59-65, Aug. 2005. 